\title{
Chiral Brønsted acid-catalyzed asymmetric allyl(propargyl)boration reaction of ortho-alkynyl benzaldehydes: synthetic applications and factors governing the enantioselectivity
}

Elsa Rodríguez, ${ }^{\mathrm{a}}$ Matthew N. Grayson, ${ }^{\mathrm{b}}$ Amparo Asensio, ${ }^{\mathrm{a}}$ Pablo Barrio, ${ }^{*, \mathrm{a}} \mathrm{K} . \mathrm{N}$. Houk $^{*, \mathrm{~b}}$ and Santos Fustero*,a,c

a Departamento de Química Orgánica, Universidad de Valencia, Av. Vicente Andrés Estellés s/n E-46100 Burjassot, Spain

${ }^{\mathrm{b}}$ Department of Chemistry and Biochemistry, University of California, Los Angeles, California $90095-$ 1569, United States

c Laboratorio de Moléculas Orgánicas, Centro de Investigación Príncipe Felipe, E-46012 Valencia, Spain

pablo.barrio@uv.es

houk@chem.ucla.edu

santos.fustero@uv.es

Table of contents:

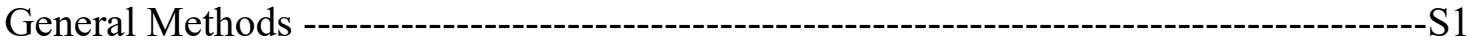

Experimental Procedures and Characterizations ---------------------------------------------S2

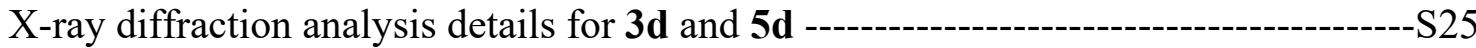

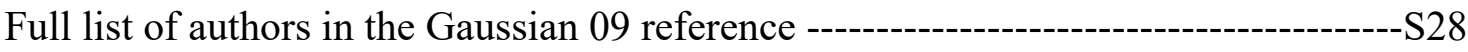

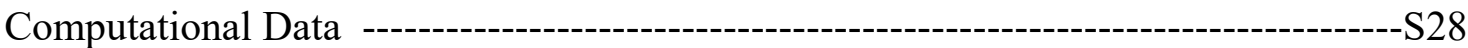

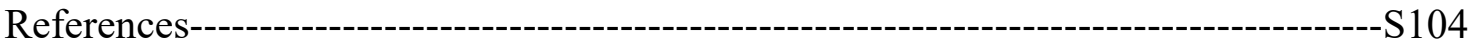

Spectra and HPLC chromatograms of Compounds ----------------------------------------S105

General methods:

All the reactions were carried out under a nitrogen atmosphere using oven dried glassware unless otherwise indicated. The solvents were purified prior to use: THF and $\mathrm{PhMe}$ were distilled from sodium / benzophenone and $\mathrm{CH}_{2} \mathrm{Cl}_{2}$ was distilled from 
calcium hydride. Cyclohexane, cyclopentane, F8-toluene, $\mathrm{F}_{3}$-toluene, $\mathrm{MeOH}$ and DCE were purchased from Aldrich as anhydrous and used as received. All reagents were used as received from the commercial supplier. The reactions were monitored with the aid of thin-layer chromatography (TLC) on $0.25 \mathrm{~mm}$ E. Merck precoated silica gel plates. Visualization was carried out with UV light and aqueous ceric ammonium molybdate solution or potassium permanganate stain. Flash column chromatography was performed with the indicated solvents on silica gel 60 (particle size $0.040-0.063 \mathrm{~mm}$ ). Melting points were measured on a Büchi B-540 apparatus and are uncorrected. Optical rotations were measured on a Perkin-Elmer 241 polarimeter. ${ }^{1} \mathrm{H},{ }^{19} \mathrm{~F}$ and ${ }^{13} \mathrm{C} \mathrm{NMR}$ spectra were recorded on a $300 \mathrm{MHz}$ Bruker AC300 spectrometer and $400 \mathrm{MHz}$ Bruker Avance. Chemical shifts are given in $\mathrm{ppm}(\delta)$, with reference to the residual proton resonances of the solvents. ${ }^{1}$ Coupling constants $(J)$ are given in Hertz $(\mathrm{Hz})$. The letters $\mathrm{m}, \mathrm{s}, \mathrm{d}, \mathrm{t}, \mathrm{q}$ and sept stand for multiplet, singlet, doublet, triplet, quartet and septet respectively. The letters br indicate that the signal is broad. High-resolution mass spectra (ESI) were carried out on VGmAutospec (VG Analytical, Micromass Instruments) by the Universidad de Valencia Mass Spectrometry Service. HPLC analyses were performed using a JASCO PU-2089 Plus chromatographer equipped with an MD-2010 detector.

Experimental Procedures and Characterizations:

a) Synthesis of starting o-alkynylbenzaldehydes compounds $\mathbf{1 a - f , h - p :}$

Starting o-alkynylbenzaldehydes compounds 1a-f,h-p were synthesized from the corresponding $o$-bromobenzaldehyde by means of a Sonogashira Coupling:

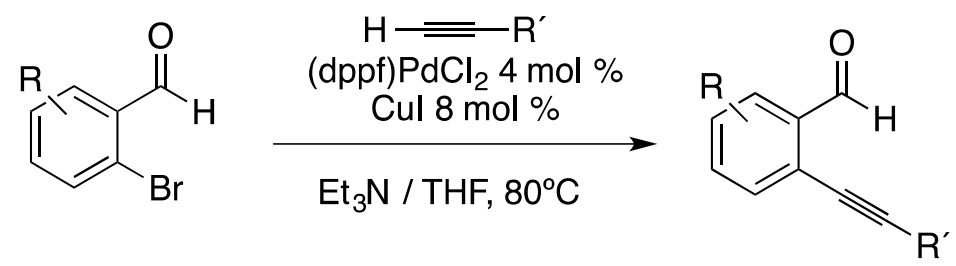

General procedure for the Sonogashira Coupling:

To a solution of the corresponding 2-bromobenzaldehyde in $\mathrm{Et}_{3} \mathrm{~N}$ :THF (10:1) (0.5 M) the corresponding alkyne (1.3 equiv), $\mathrm{CuI}(8 \mathrm{~mol} \%)$ and (dppf) $\mathrm{PdCl}_{2}(4 \mathrm{~mol} \%)$ were added and the reaction mixture was then heated at $80^{\circ} \mathrm{C}$. Upon consumption of the starting material ( $3 \mathrm{~h}$ approximately), the reaction was filtered, quenched with water and extracted with AcOEt. The organic layers were combined, dried over $\mathrm{Na}_{2} \mathrm{SO}_{4}$, filtered 
and evaporated under reduced pressure. The crude product was purified by flash chromatography employing mixtures of $n$-hexane:ethyl acetate as eluents.

2-((3,5-Difluorophenyl)ethynyl)benzaldehyde (1k):

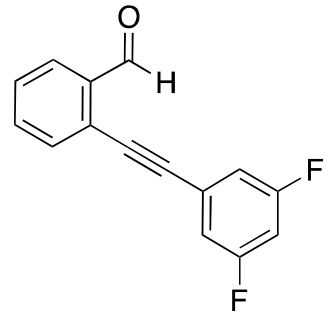

Flash chromatography of the crude reaction product [ $n$-hexane:EtOAc $(20: 1)]$ gave a white solid (65\%). mp 63-65 ${ }^{\circ} \mathrm{C} .{ }^{1} \mathrm{H}$ NMR $\left(300 \mathrm{MHz}, \mathrm{CDCl}_{3}\right) \delta 6.85$ (tt, $J_{1}=8.9 J_{2}=2.3$, 1H), 7.04-7.11 (m, 2H), 7.47-7.53 (m, 1H), 7.58-7.66 (m, 2H), 7.94-7.97 (m, 1H), 10.57 $(\mathrm{d}, J=0.8,1 \mathrm{H}) .{ }^{19} \mathrm{~F}$ NMR $\left(282.4 \mathrm{MHz}, \mathrm{CDCl}_{3}\right) \delta-109.5(\mathrm{~m}) .{ }^{13} \mathrm{C}$ NMR $(75.5 \mathrm{MHz}$, $\left.\mathrm{CDCl}_{3}\right) \delta 86.8(\mathrm{C}), 93.6\left(\mathrm{t},{ }^{4} J_{\mathrm{CF}}=4.0, \mathrm{C}\right), 105.2\left(\mathrm{t},{ }^{2} J_{\mathrm{CF}}=25.3, \mathrm{CH}\right), 114.6\left(\mathrm{~d},{ }^{2} J_{\mathrm{CF}}=17.9\right.$, $\mathrm{CH}), 114.7\left(\mathrm{~d},{ }^{2} J_{\mathrm{CF}}=17.8, \mathrm{CH}\right), 125.0\left(\mathrm{t},{ }^{3} J_{\mathrm{CF}}=11.5, \mathrm{C}\right), 125.5(\mathrm{C}), 127.7(\mathrm{CH}), 129.3$ $(\mathrm{CH}), 133.4(\mathrm{CH}), 133.8(\mathrm{CH}), 136.0(\mathrm{C}), 162.7$ (d, $\left.{ }^{1} J_{\mathrm{CF}}=249.4, \mathrm{C}\right), 162.8$ (d, $\left.{ }^{1} J_{\mathrm{CF}}=249.6, \mathrm{C}\right), 191.0(\mathrm{CH})$. HRMS (ESI) calcd for $\mathrm{C}_{15} \mathrm{H}_{8} \mathrm{~F}_{2} \mathrm{O}[\mathrm{M}+\mathrm{H}]^{+}: 243.0616$, found: 243.0614.

\section{4-Fluoro-2-(phenylethynyl)benzaldehyde (10):}<smiles>O=Cc1ccc(F)cc1C#Cc1ccccc1</smiles>

Flash chromatography of the crude reaction product [ $n$-hexane:EtOAc (20:1)] gave a white solid (73\%). mp 34-36 ${ }^{\circ} \mathrm{C} .{ }^{1} \mathrm{H}$ NMR $\left(300 \mathrm{MHz}, \mathrm{CDCl}_{3}\right) \delta 7.11-7.18(\mathrm{~m}, 1 \mathrm{H}), 7.32$ $\left(\mathrm{dd}, J_{1}=9.0 J_{2}=2.5,1 \mathrm{H}\right), 7.38-7.43(\mathrm{~m}, 3 \mathrm{H}), 7.55-7.59(\mathrm{~m}, 2 \mathrm{H}), 7.98\left(\mathrm{dd}, J_{1}=8.7 J_{2}=5.9\right.$, $1 \mathrm{H}), 10.57\left(\mathrm{~d},{ }^{6} J_{\mathrm{FH}}=0.8,1 \mathrm{H}\right) .{ }^{19} \mathrm{~F}$ NMR $\left(282.4 \mathrm{MHz}, \mathrm{CDCl}_{3}\right) \delta-103.3\left(\mathrm{td},{ }^{3} J_{\mathrm{FH}}=8.4\right.$ $\left.{ }^{4} J_{\mathrm{FH}}=6.1\right) .{ }^{13} \mathrm{C} \mathrm{NMR}\left(75.5 \mathrm{MHz}, \mathrm{CDCl}_{3}\right) \delta 83.7\left(\mathrm{~d},{ }^{4} J_{\mathrm{CF}}=3.1, \mathrm{C}\right), 97.4(\mathrm{C}), 116.5(\mathrm{~d}$, $\left.{ }^{2} J_{\mathrm{CF}}=22.2, \mathrm{CH}\right), 119.7\left(\mathrm{~d},{ }^{2} J_{\mathrm{CF}}=23.4, \mathrm{CH}\right), 121.8(\mathrm{C}), 128.6(2 \mathrm{xCH}), 129.3(\mathrm{C}), 129.4$ $(\mathrm{CH}), 130.1\left(\mathrm{~d},{ }^{3} J_{\mathrm{CF}}=10.3, \mathrm{CH}\right), 131.8(2 \mathrm{xCH}), 132.6\left(\mathrm{~d},{ }^{4} J_{\mathrm{CF}}=3.1, \mathrm{C}\right), 165.7(\mathrm{~d}$, $\left.{ }^{1} J_{\mathrm{CF}}=257.0, \mathrm{C}\right), 190.0(\mathrm{CH})$. HRMS (ESI) calcd for $\mathrm{C}_{15} \mathrm{H}_{9} \mathrm{FO}[\mathrm{M}+\mathrm{H}]^{+}: 225.0710$, found: 225.0707 .

The Sonogashira products $\mathbf{1 a - f}, \mathbf{1 h}-\mathbf{j}, \mathbf{1} \mathbf{l}-\mathbf{n}, \mathbf{1 p}$ have already been described following the same methodology. ${ }^{2}$ 
b) Synthesis of starting o-alkynylbenzaldehyde compound $\mathbf{1 g}$ :

The $o$-alkynylbenzaldehyde $\mathbf{1 g}$ was synthesized following this reaction sequence:<smiles></smiles>

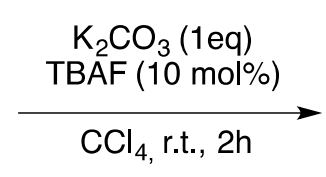<smiles>ClC#Cc1ccccc1C1OCCO1</smiles>

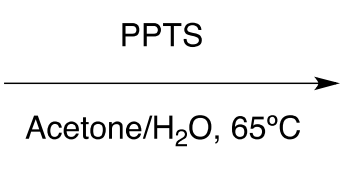<smiles>O=Cc1ccccc1C#CCl</smiles>

Procedure for the chlorination reaction:

To a solution of compound $\boldsymbol{I}$ in $\mathrm{CCl}_{4}(2 \mathrm{M}), \mathrm{K}_{2} \mathrm{CO}_{3}$ (1 equiv.) and TBAF (10 mol\%) were added. After stirring for 2 hours at room temperature, the reaction mixture was quenched with saturated ammonium chloride solution, followed by extraction with diethyl ether. The combined organic layers were dried over $\mathrm{Na}_{2} \mathrm{SO}_{4}$, filtered and evaporated under reduced pressure. The crude product was purified by flash chromatography employing a mixture of $n$-hexane:ethyl acetate $(20: 1)$ as eluent.

\section{2-(2-(Chloroethynyl)phenyl)-1,3-dioxolane (II):}<smiles>ClC#Cc1ccccc1C1OCCO1</smiles>

Flash chromatography of the crude reaction product [n-hexane:EtOAc (20:1)] gave a colorless oil (96\%). ${ }^{1} \mathrm{H}$ NMR $\left(300 \mathrm{MHz}, \mathrm{CDCl}_{3}\right) \delta 3.98-4.10(\mathrm{~m}, 4 \mathrm{H}), 6.05(\mathrm{~s}, 1 \mathrm{H})$, 7.23-7.49 (m, 4H). ${ }^{13} \mathrm{C}$ NMR (75.5 MHz, $\left.\mathrm{CDCl}_{3}\right) \delta 65.6\left(2 \mathrm{xCH}_{2}\right), 66.9(\mathrm{C}), 72.2(\mathrm{C})$, $101.9(\mathrm{CH}), 121.3(\mathrm{C}), 126.3(\mathrm{CH}), 128.7(\mathrm{CH}), 129.0(\mathrm{CH}), 133.1(\mathrm{CH}), 139.7(\mathrm{C})$. HRMS (ESI) calcd for $\mathrm{C}_{11} \mathrm{H}_{9} \mathrm{ClO}_{2}[\mathrm{M}+\mathrm{H}]^{+}:$209.0364, found: 209.0360 .

Procedure for the deprotection reaction:

To a solution of compound $\boldsymbol{I I}$ in a mixture of acetone: $\mathrm{H}_{2} \mathrm{O}$ 10:1 (0.1M), PPTS (20 mol\%) was added. The reaction mixture was heated at $65^{\circ} \mathrm{C}$ for 4 hours. After this time, the acetone was evaporated under reduced pressure, the reaction mixture was diluted with ethyl acetate and the two layers were separated. The aqueous layer was extracted with ethyl acetate. The combined organic layers were dried over $\mathrm{Na}_{2} \mathrm{SO}_{4}$, filtered and evaporated under reduced pressure. The crude product was purified by flash chromatography employing a mixture of $n$-hexane:ethyl acetate $(20: 1)$ as eluent. 


\section{2-(Chloroethynyl)benzaldehyde (1g):}<smiles>O=Cc1ccccc1C#CCl</smiles>

Flash chromatography of the crude reaction product [ $n$-hexane:EtOAc (20:1)] gave a yellow solid (95\%). mp 32-34 ${ }^{\circ} \mathrm{C} .{ }^{1} \mathrm{H}$ NMR $\left(300 \mathrm{MHz}, \mathrm{CDCl}_{3}\right) \delta$ 7.43-7.49 (m, 1H), 7.54-7.58 (m, 2H), 7.89-7.92 (m, 1H), $10.46(\mathrm{~d}, J=0.8,1 \mathrm{H}) .{ }^{13} \mathrm{C}$ NMR (75.5 MHz, $\left.\mathrm{CDCl}_{3}\right) \delta 65.4(\mathrm{C}), 75.0(\mathrm{C}), 125.5(\mathrm{C}), 127.4(\mathrm{CH}), 128.9(\mathrm{CH}), 133.7(\mathrm{CH}), 133.8$ $(\mathrm{CH}), 136.7(\mathrm{C}), 191.1(\mathrm{CH})$. HRMS (ESI) calcd for $\mathrm{C}_{9} \mathrm{H}_{5} \mathrm{ClO}[\mathrm{M}+\mathrm{H}]^{+}$: 165.0102 , found: 165.0093 .

c) General procedure for the enantioselective allylboration:

\section{Conditions A:}

To a solution of the corresponding 2-alkynylbenzaldehyde 1a-p (typically $0.1 \mathrm{mmol}$ ) in toluene $(0.1 \mathrm{M}),(R)$-TRIP-PA $(5 \mathrm{~mol} \%)$ was added and the reaction mixture was cooled to $-30^{\circ} \mathrm{C}$ followed by the addition of the allylboronic acid pinacol ester 2 (1.2 equiv.). Upon completion (approximately $1 \mathrm{~h}$ ), solvents were removed under reduced pressure and the residue purified by flash chromatography using mixtures of $n$-hexane:ethyl acetate as eluent. The enantiomeric excess of the product was determined by HPLC: Chiralcel OD-H (25 cm x $0.46 \mathrm{~cm}$ column), hexane:isopropanol 98:2 as eluent and flow $=1 \mathrm{ml} / \mathrm{min}$, unless otherwise indicated.

\section{Conditions B:}

Analogous procedure to conditions A but using DCM as solvent.

\section{Optimized conditions:}

Analogous procedure to conditions A but using DCM as solvent and treating the reaction mixture with activated $4 \AA \mathrm{MS}(50 \mathrm{mg} / 0.1 \mathrm{mmol}$ of $\mathbf{1})$ for 5 minutes before the addition of the allylboronic acid pinacol ester $\mathbf{2}$. 


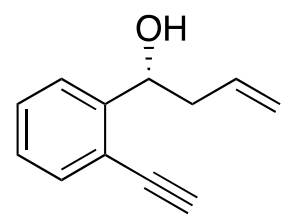

Following conditions $\mathrm{B}$ for the asymmetric allylboration of aldehyde 1a, the title compound was obtained in $91 \%$ yield and $62 \%$ ee with properties identical to those reported in the literature. ${ }^{3}$ The enantiomeric excess was determined with the aid of HPLC analysis: Chiralpak IC (25 cm x $0.46 \mathrm{~cm}$ column), hexane:isopropanol 99.5:0.5 as eluent and flow $=0.8 \mathrm{~mL} / \mathrm{min}: t_{\mathrm{R} \text { minor }}=46.1 \mathrm{~min}, t_{\mathrm{R} \text { major }}=49.3 \mathrm{~min}$.

(R)-1-(2-(Hex-1-ynyl)phenyl)but-3-en-1-ol (3b):<smiles>C=CCC(O)c1ccccc1C#CBr</smiles>

Flash chromatography of the crude reaction product [n-hexane:EtOAc (10:1)] gave a colorless oil (conditions A: 78\%, 79\% ee). ${ }^{1} \mathrm{H}$ NMR (300 MHz, $\left.\mathrm{CDCl}_{3}\right) \delta 0.96$ (t, J=7.2, $3 \mathrm{H}), 1.43-1.65$ (m, 4H), 2.20 (br s, OH), 2.39-2.49 (m, 3H), 2.61-2.70 (m, 1H), 5.13$5.21(\mathrm{~m}, 3 \mathrm{H}), 5.80-5.94(\mathrm{~m}, 1 \mathrm{H}), 7.19\left(\mathrm{td}, J_{1}=7.5 J_{2}=1.4,1 \mathrm{H}\right), 7.29\left(\mathrm{td}, J_{1}=7.6 J_{2}=1.5\right.$, $1 \mathrm{H}), 7.38\left(\mathrm{dd}, J_{1}=7.5 J_{2}=1.5,1 \mathrm{H}\right), 7.46-7.49(\mathrm{~m}, 1 \mathrm{H}) .{ }^{13} \mathrm{C} \mathrm{NMR}\left(75.5 \mathrm{MHz}, \mathrm{CDCl}_{3}\right) \delta$ $\left.13.6\left(\mathrm{CH}_{3}\right), 19.2\left(\mathrm{CH}_{2}\right), 22.0\left(\mathrm{CH}_{2}\right), 30.8 \mathrm{CH}_{2}\right), 42.6\left(\mathrm{CH}_{2}\right), 71.3(\mathrm{CH}), 78.2(\mathrm{C}), 95.8$ (C), $118.0\left(\mathrm{CH}_{2}\right), 121.2(\mathrm{C}), 125.1(\mathrm{CH}), 126.9(\mathrm{CH}), 127.8(\mathrm{CH}), 132.2(\mathrm{CH}), 134.8$ (CH), 145.4 (C). HRMS (ESI) calcd for $\mathrm{C}_{16} \mathrm{H}_{20} \mathrm{O}[\mathrm{M}-\mathrm{OH}]^{+}: 211.1481$, found: 211.1476. The enantiomeric excess was determined with the aid of HPLC analysis: Chiralpak IC (25 cm x $0.46 \mathrm{~cm}$ column), hexane:isopropanol 99:1 as eluent and flow $=0.8 \mathrm{~mL} / \mathrm{min}$ : $t_{\mathrm{R} \text { minor }}=18.1 \mathrm{~min}, t_{\mathrm{R} \text { major }}=30.7 \mathrm{~min}$.

(R)-1-(2-(Cyclohexylethynyl)phenyl)but-3-en-1-ol (3c):<smiles>C=CCC(O)c1ccccc1C#CCCl</smiles> 
Flash chromatography of the crude reaction product [n-hexane:EtOAc (10:1)] gave a colorless oil (conditions B: 98\%, 76\% ee). ${ }^{1} \mathrm{H}$ NMR (300 MHz, $\left.\mathrm{CDCl}_{3}\right) \delta 1.27-1.34$ (m, $3 \mathrm{H}), 1.44-1.53$ (m, 3H), 1.65-1.73 (m, 2H), 1.79-1.85 (m, 2H), 2.21 (br s, OH), 2.32$2.43(\mathrm{~m}, 1 \mathrm{H}), 2.53-2.65(\mathrm{~m}, 2 \mathrm{H}), 5.06-5.15(\mathrm{~m}, 3 \mathrm{H}), 5.73-5.87(\mathrm{~m}, 1 \mathrm{H}), 7.12\left(\mathrm{td}, J_{1}=7.5\right.$ $\left.J_{2}=1.4,1 \mathrm{H}\right), 7.22\left(\mathrm{td}, J_{1}=7.6 J_{2}=1.5,1 \mathrm{H}\right), 7.31\left(\mathrm{dd}, J_{1}=7.6 J_{2}=1.3,1 \mathrm{H}\right), 7.40\left(\mathrm{dd}, J_{1}=7.8\right.$ $\left.J_{2}=1.3,1 \mathrm{H}\right) .{ }^{13} \mathrm{C} \mathrm{NMR}\left(75.5 \mathrm{MHz}, \mathrm{CDCl}_{3}\right) \delta 24.8\left(2 \mathrm{xCH}_{2}\right), 25.9\left(\mathrm{CH}_{2}\right), 29.7(\mathrm{CH}), 32.6$ $\left(2 \mathrm{xCH}_{2}\right), 42.6\left(\mathrm{CH}_{2}\right), 71.4(\mathrm{CH}), 78.2(\mathrm{C}), 99.9(\mathrm{C}), 118.1\left(\mathrm{CH}_{2}\right), 121.2(\mathrm{C}), 125.1$ (CH), $126.9(\mathrm{CH}), 127.8(\mathrm{CH}), 132.1(\mathrm{CH}), 134.9(\mathrm{CH}), 145.4(\mathrm{C})$. HRMS (ESI) calcd for $\mathrm{C}_{18} \mathrm{H}_{22} \mathrm{O}[\mathrm{M}-\mathrm{H}]^{+}:$253.1587, found: 253.1589. The enantiomeric excess was determined with the aid of HPLC analysis: Chiralpak IC ( $25 \mathrm{~cm} \times 0.46 \mathrm{~cm}$ column), hexane:isopropanol 99.1:0.9 as eluent and flow $=0.8 \mathrm{~mL} / \mathrm{min}: t_{\mathrm{R}}$ minor $=16.3 \mathrm{~min}, t_{\mathrm{R}}$ major $=26.1 \mathrm{~min}$.

(R)-1-(2-(Phenylethynyl)phenyl)but-3-en-1-ol (3d):<smiles>C=CCC(O)c1ccccc1C#Cc1ccccc1</smiles>

Following the optimized conditions for the asymmetric allylboration of aldehyde $\mathbf{1 d}$, the title compound was obtained in $99 \%$ and $99 \%$ ee with properties identical to those reported in the literature. ${ }^{4}$ The enantiomeric excess was determined as described in conditions $\mathrm{A}: t_{\mathrm{R} \text { minor }}=14.4 \mathrm{~min}, t_{\mathrm{R} \text { major }}=17.5 \mathrm{~min} .[\alpha]_{\mathrm{D}}{ }^{25}=+129.0\left(\mathrm{c} 1.0 ; \mathrm{CHCl}_{3}\right)$.

(R)-1-(2-((Trimethylsilyl)ethynyl)phenyl)but-3-en-1-ol (3e):<smiles>C=CCC(O)c1ccccc1C#CC(C)(C)C</smiles>

Flash chromatography of the crude reaction product [n-hexane:EtOAc (10:1)] gave a colorless oil (conditions B: 99\%, 53\% ee). ${ }^{1} \mathrm{H}$ NMR (300 MHz, $\left.\mathrm{CDCl}_{3}\right) \delta 0.26(\mathrm{~s}, 9 \mathrm{H})$, 2.16 (br s, OH), 2.37-2.48 (m, 1H), 2.63-2.72 (m, 1H), 5.14-5.22 (m, 3H), 5.87 (dddd, $\left.J_{1}=16.8 J_{2}=10.2 \quad J_{3}=7.7 \quad J_{4}=6.5,1 \mathrm{H}\right), 7.20\left(\mathrm{td}, J_{1}=7.5 \quad J_{2}=1.3,1 \mathrm{H}\right), 7.34\left(\mathrm{td}, J_{1}=7.6\right.$ $\left.J_{2}=1.3,1 \mathrm{H}\right), 7.43-7.52(\mathrm{~m}, 2 \mathrm{H}) .{ }^{13} \mathrm{C} \mathrm{NMR}\left(75.5 \mathrm{MHz}, \mathrm{CDCl}_{3}\right) \delta-0.1\left(3 \mathrm{xCH}_{3}\right), 42.7$ $\left(\mathrm{CH}_{2}\right), 71.3(\mathrm{CH}), 99.9(\mathrm{C}), 102.7(\mathrm{C}), 118.2\left(\mathrm{CH}_{2}\right), 120.2(\mathrm{C}), 125.2(\mathrm{CH}), 126.9(\mathrm{CH})$, 
$128.9(\mathrm{CH}), 132.4(\mathrm{CH}), 134.7(\mathrm{CH}), 146.2(\mathrm{C})$. HRMS (ESI) calcd for $\mathrm{C}_{15} \mathrm{H}_{20} \mathrm{OSi}$ $[\mathrm{M}+\mathrm{H}]^{+}: 245.1356$, found: 245.1357 . The enantiomeric excess was determined with the aid of HPLC analysis: Chiralpak IC (25 cm x $0.46 \mathrm{~cm}$ column), hexane:isopropanol 99.5:0.5 as eluent and flow $=0.8 \mathrm{~mL} / \mathrm{min}: t_{\mathrm{R} \text { minor }}=45.6 \mathrm{~min}, t_{\mathrm{R} \text { major }}=58.3 \mathrm{~min}$.

(R)-1-(2-((Triisopropylsilyl)ethynyl)phenyl)but-3-en-1-ol (3f):

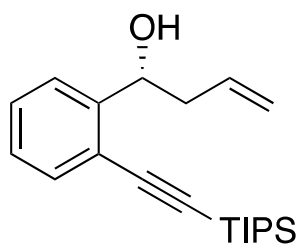

Flash chromatography of the crude reaction product [ $n$-hexane:EtOAc (20:1)] gave a colorless oil (conditions B: 91\%, 5\% ee). ${ }^{1} \mathrm{H}$ NMR $\left(300 \mathrm{MHz}, \mathrm{CDCl}_{3}\right) \delta 1.14(\mathrm{~s}, 21 \mathrm{H})$, $2.30(\mathrm{~d}, J=3.9,1 \mathrm{H}), 2.39-2.50(\mathrm{~m}, 1 \mathrm{H}), 2.66-2.75(\mathrm{~m}, 1 \mathrm{H}), 5.14-5.21(\mathrm{~m}, 2 \mathrm{H}), 5.22-5.28$ $(\mathrm{m}, 1 \mathrm{H}), 5.78-5.87(\mathrm{~m}, 1 \mathrm{H}), 7.21\left(\mathrm{td}, J_{1}=7.5 J_{2}=1.4,1 \mathrm{H}\right), 7.34\left(\mathrm{td}, J_{1}=7.6 J_{2}=1.5,1 \mathrm{H}\right)$, 7.46-7.52 (m, 2H). $\left.{ }^{13} \mathrm{C} \mathrm{NMR} \mathrm{(75.5} \mathrm{MHz,} \mathrm{CDCl}_{3}\right) \delta 11.3(3 \mathrm{xCH}), 18.7\left(6 \mathrm{xCH}_{3}\right), 42.8$ $\left(\mathrm{CH}_{2}\right), 71.2(\mathrm{CH}), 96.1(\mathrm{C}), 104.5(\mathrm{C}), 118.4\left(\mathrm{CH}_{2}\right), 120.6(\mathrm{C}), 125.2(\mathrm{CH}), 126.9(\mathrm{CH})$, $128.7(\mathrm{CH}), 133.0(\mathrm{CH}), 134.5(\mathrm{CH}), 145.9(\mathrm{C})$. HRMS (ESI) calcd for $\mathrm{C}_{21} \mathrm{H}_{32} \mathrm{OSi}$ $[\mathrm{M}+\mathrm{H}]^{+}:$329.2295, found: 329.2302 . The enantiomeric excess was determined with the aid of HPLC analysis: Chiralpak IC (25 cm x $0.46 \mathrm{~cm}$ column), hexane as eluent and flow $=0.8 \mathrm{~mL} / \mathrm{min}: t_{\mathrm{R} \text { minor }}=31.8 \mathrm{~min}, t_{\mathrm{R} \text { major }}=36.0 \mathrm{~min}$.

(R)-1-(2-(Chloroethynyl)phenyl)but-3-en-1-ol (3g):<smiles>C=CCC(O)c1ccccc1C#CCl</smiles>

Flash chromatography of the crude reaction product [ $n$-hexane:EtOAc (10:1)] gave a white solid (conditions B: 99\%, 90\% ee). $[\alpha]_{\mathrm{D}}^{25}=+79.7\left(\right.$ c 1.0; $\left.\mathrm{CHCl}_{3}\right)$. mp 39-41 ${ }^{\circ} \mathrm{C}$. ${ }^{1} \mathrm{H}$ NMR $\left(300 \mathrm{MHz}, \mathrm{CDCl}_{3}\right) \delta$ 2.10-2.13 (m, 1H), 2.37-2.47 (m, 1H), 2.57-2.67 (m, $1 \mathrm{H}), 5.13-5.21(\mathrm{~m}, 3 \mathrm{H}), 5.80-5.91(\mathrm{~m}, 1 \mathrm{H}), 7.22\left(\mathrm{td}, J_{1}=7.5 J_{2}=1.4,1 \mathrm{H}\right), 7.37\left(\mathrm{td}, J_{1}=7.6\right.$ $\left.J_{2}=1.4,1 \mathrm{H}\right), 7.41-7.44(\mathrm{~m}, 1 \mathrm{H}), 7.51-7.54(\mathrm{~m}, 1 \mathrm{H}) .{ }^{13} \mathrm{C} \mathrm{NMR}\left(75.5 \mathrm{MHz}, \mathrm{CDCl}_{3}\right) \delta 42.9$ $\left(\mathrm{CH}_{2}\right), 61.2(\mathrm{C}), 71.0(\mathrm{CH}), 72.62(\mathrm{C}), 118.5\left(\mathrm{CH}_{2}\right), 119.4(\mathrm{C}), 125.3(\mathrm{CH}), 127.1(\mathrm{CH})$, $129.0(\mathrm{CH}), 132.7(\mathrm{CH}), 134.4(\mathrm{CH}), 146.3(\mathrm{C})$. HRMS (EI) calcd for $\mathrm{C}_{12} \mathrm{H}_{11} \mathrm{ClO}$ [M- 
$\mathrm{OH}]^{+}: 189.0466$, found: 189.0455 . The enantiomeric excess was determined with the aid of HPLC analysis: Chiralpak IC ( $25 \mathrm{~cm} \times 0.46 \mathrm{~cm}$ column), hexane:isopropanol 99:1 as eluent and flow $=1 \mathrm{~mL} / \mathrm{min}: t_{\mathrm{R} \text { minor }}=12.1 \mathrm{~min}, t_{\mathrm{R} \text { major }}=14.3 \mathrm{~min}$.

(R)-1-(2-((2-Methoxyphenyl)ethynyl)phenyl)but-3-en-1-ol (3h):<smiles>C=CCC(O)c1ccccc1C#Cc1ccccc1OC</smiles>

Flash chromatography of the crude reaction product [n-hexane:EtOAc (10:1)] gave a colorless oil (optimized conditions: $91 \%, 85 \%$ ee). $[\alpha]_{\mathrm{D}}{ }^{25}=+37.9\left(\right.$ c $\left.1.0 ; \mathrm{CHCl}_{3}\right) .{ }^{1} \mathrm{H}$ NMR (300 MHz, $\left.\mathrm{CDCl}_{3}\right) \delta$ 2.59-2.70 (m, 1H), 2.73-2.82 (m, 1H), $3.05(\mathrm{~d}, J=6.0,1 \mathrm{H})$, $3.92(\mathrm{~s}, 3 \mathrm{H}), 5.10-5.21(\mathrm{~m}, 3 \mathrm{H}), 5.85-5.98(\mathrm{~m}, 1 \mathrm{H}), 6.92(\mathrm{~d}, J=8.8,1 \mathrm{H}), 6.97\left(\mathrm{td}, J_{1}=7.5\right.$ $\left.J_{2}=1.0,1 \mathrm{H}\right), 7.25\left(\mathrm{td}, J_{1}=7.4 J_{2}=1.5,1 \mathrm{H}\right), 7.30-7.36(\mathrm{~m}, 2 \mathrm{H}), 7.43-7.46(\mathrm{~m}, 1 \mathrm{H}), 7.47-$ $7.50(\mathrm{~m}, 1 \mathrm{H}), 7.53-7.56(\mathrm{~m}, 1 \mathrm{H}) .{ }^{13} \mathrm{C} \mathrm{NMR}\left(75.5 \mathrm{MHz}, \mathrm{CDCl}_{3}\right) \delta 42.2\left(\mathrm{CH}_{2}\right), 55.7$ $\left(\mathrm{CH}_{3}\right), 72.5(\mathrm{CH}), 91.1(\mathrm{C}), 91.6(\mathrm{C}), 110.5(\mathrm{CH}), 112.3(\mathrm{C}), 117.7\left(\mathrm{CH}_{2}\right), 120.6(\mathrm{CH})$, $120.7(\mathrm{C}), 126.0(\mathrm{CH}), 127.0(\mathrm{CH}), 128.4(\mathrm{CH}), 129.9(\mathrm{CH}), 132.3(\mathrm{CH}), 132.8(\mathrm{CH})$, $135.1(\mathrm{CH}), 145.8(\mathrm{C}), 160.0$ (C). HRMS (EI) calcd for $\mathrm{C}_{19} \mathrm{H}_{18} \mathrm{O}_{2}[\mathrm{M}-\mathrm{OH}]^{+}: 261.1274$, found: 261.1273. The enantiomeric excess was determined with the aid of HPLC analysis: Chiralcel OD-H (25 cm x 0.46 cm column), hexane:isopropanol 95:5 as eluent and flow $=1 \mathrm{~mL} / \mathrm{min}: t_{\mathrm{R} \text { minor }}=14.1 \mathrm{~min}, t_{\mathrm{R} \text { major }}=16.6 \mathrm{~min}$.

(R)-1-(2-((4-(Trifluoromethyl)phenyl)ethynyl)phenyl)but-3-en-1-ol (3i):<smiles>C=CCC(O)c1ccccc1C#Cc1ccc(C(F)(F)F)cc1</smiles>

Flash chromatography of the crude reaction product [ $n$-hexane:EtOAc (15:1)] gave a white solid (optimized conditions: $95 \%, 88 \%$ ee). $[\alpha]_{\mathrm{D}}^{25}=+77.2$ (c 1.0; $\mathrm{CHCl}_{3}$ ). mp 83$85{ }^{\circ} \mathrm{C} .{ }^{1} \mathrm{H}$ NMR $\left(300 \mathrm{MHz}, \mathrm{CDCl}_{3}\right) \delta$ 2.20-2.23 (m, 1H), 2.45-2.55 (m, 1H), 2.67-2.76 $(\mathrm{m}, 1 \mathrm{H}), 5.16-5.24(\mathrm{~m}, 2 \mathrm{H}), 5.27-5.32(\mathrm{~m}, 1 \mathrm{H}), 5.84-5.92(\mathrm{~m}, 1 \mathrm{H}), 7.30\left(\mathrm{td}, J_{1}=7.5\right.$ 
$\left.J_{2}=1.4,1 \mathrm{H}\right), 7.41\left(\mathrm{td}, J_{1}=7.6 J_{2}=1.4,1 \mathrm{H}\right), 7.52-7.55(\mathrm{~m}, 1 \mathrm{H}), 7.58-7.64(\mathrm{~m}, 5 \mathrm{H}) .{ }^{19} \mathrm{~F}$ NMR (282.4 MHz, $\left.\mathrm{CDCl}_{3}\right) \delta-62.3(\mathrm{~s}) .{ }^{13} \mathrm{C} \mathrm{NMR}\left(75.5 \mathrm{MHz}, \mathrm{CDCl}_{3}\right) \delta 43.0\left(\mathrm{CH}_{2}\right), 71.3$ $(\mathrm{CH}), 89.4(\mathrm{C}), 93.0(\mathrm{C}), 118.5\left(\mathrm{CH}_{2}\right), 119.7(\mathrm{C}), 123.9\left(\mathrm{q},{ }^{1} J_{\mathrm{CF}}=272.1, \mathrm{CF}_{3}\right), 125.4$ $(\mathrm{CH}), 125.4$ (q, $\left.{ }^{3} J_{\mathrm{CF}}=3.6,2 x \mathrm{xH}\right), 126.9(\mathrm{C}), 127.2(\mathrm{CH}), 129.3(\mathrm{CH}), 130.1$ (q, $\left.{ }^{2} J_{\mathrm{CF}}=33.0, \mathrm{C}\right), 131.6(2 \mathrm{xCH}), 132.3(\mathrm{CH}), 134.5(\mathrm{CH}), 146.0(\mathrm{C})$. HRMS (EI) calcd for $\mathrm{C}_{19} \mathrm{H}_{15} \mathrm{~F}_{3} \mathrm{O}[\mathrm{M}-\mathrm{OH}]^{+}:$299.1042, found: 299.1044. The enantiomeric excess was determined with the aid of HPLC analysis: Chiralpak IC (25 cm x $0.46 \mathrm{~cm}$ column), hexane:isopropanol 99.1:0.9 as eluent and flow $=1 \mathrm{~mL} / \mathrm{min}: t_{\mathrm{R} \text { minor }}=24.6 \mathrm{~min}, t_{\mathrm{R} \text { major }}=$ $26.2 \mathrm{~min}$.

(R)-1-(2-((2-Nitrophenyl)ethynyl)phenyl)but-3-en-1-ol (3j):<smiles>C=CC[C@H](O)c1ccccc1C#Cc1ccccc1[N+](=O)[O-]</smiles>

Flash chromatography of the crude reaction product [ $n$-hexane:EtOAc (5:1)] gave a green oil (optimized conditions: $77 \%, 90 \%$ ee). $[\alpha]_{\mathrm{D}}{ }^{25}=+79.3\left(\right.$ c $\left.1.0 ; \mathrm{CHCl}_{3}\right) .{ }^{1} \mathrm{H} \mathrm{NMR}$ (300 MHz, $\left.\mathrm{CDCl}_{3}\right) \delta 2.29(\mathrm{~d}, J=3.5,1 \mathrm{H}), 2.36-2.47(\mathrm{~m}, 1 \mathrm{H}), 2.63-2.71(\mathrm{~m}, 1 \mathrm{H}), 5.07-$ $5.08(\mathrm{~m}, 1 \mathrm{H}), 5.11-5.15(\mathrm{~m}, 1 \mathrm{H}), 5.26-5.32(\mathrm{~m}, 1 \mathrm{H}), 5.82-5.96(\mathrm{~m}, 1 \mathrm{H}), 7.18-7.24(\mathrm{~m}$, 1H), 7.32-7.43 (m, 2H), 7.49-7.57 (m, 3H), 7.63-7.66 (m, 1H), 8.02-8.05 (m, 1H). ${ }^{13} \mathrm{C}$ NMR (75.5 MHz, $\left.\mathrm{CDCl}_{3}\right) \delta 43.1\left(\mathrm{CH}_{2}\right), 70.8(\mathrm{CH}), 89.4(\mathrm{C}), 95.0(\mathrm{C}), 118.5\left(\mathrm{CH}_{2}\right)$, $118.7(\mathrm{C}), 119.6(\mathrm{C}), 124.8(\mathrm{CH}), 125.6(\mathrm{CH}), 127.2(\mathrm{CH}), 128.7(\mathrm{CH}), 129.7(\mathrm{CH})$, $132.9(2 \mathrm{xCH}), 134.7(\mathrm{CH}), 134.7(\mathrm{CH}), 146.5(\mathrm{C}), 149.1$ (C). HRMS (EI) calcd for $\mathrm{C}_{18} \mathrm{H}_{15} \mathrm{NO}_{3}[\mathrm{M}-\mathrm{OH}]^{+}:$276.1019, found: 276.1022. The enantiomeric excess was determined as described in procedure c, conditions $A$ (page 5): $t_{\mathrm{R} \text { minor }}=18.0 \mathrm{~min}, t_{\mathrm{R} \text { major }}$ $=16.6 \mathrm{~min}$ 


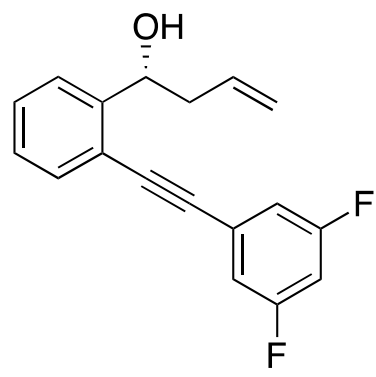

Flash chromatography of the crude reaction product [n-hexane:EtOAc (10:1)] gave a white solid (optimized conditions: $94 \%, 83 \%$ ee). $[\alpha]_{\mathrm{D}}^{25}=+85.3$ (c 1.0; $\mathrm{CHCl}_{3}$ ). mp 80$83{ }^{\circ} \mathrm{C} .{ }^{1} \mathrm{H}$ NMR $\left(300 \mathrm{MHz}, \mathrm{CDCl}_{3}\right) \delta 2.22$ (br s, OH), 2.43-2.54 (m, 1H), 2.65-2.74 (m, $1 \mathrm{H}), 5.17(\mathrm{~m}, 1 \mathrm{H}), 5.20-5.28(\mathrm{~m}, 2 \mathrm{H}), 5.85-5.91(\mathrm{~m}, 1 \mathrm{H}), 6.82\left(\mathrm{tt}, J_{1}=8.9 J_{2}=2.4,1 \mathrm{H}\right)$, 6.99-7.05 (m, 2H), $7.27\left(\mathrm{td}, J_{1}=7.5 J_{2}=1.3,1 \mathrm{H}\right), 7.38-7.44(\mathrm{~m}, 1 \mathrm{H}), 7.49-7.52(\mathrm{~m}, 1 \mathrm{H})$, 7.57-7.60 (m, 1H). ${ }^{19} \mathrm{~F}$ NMR $\left(282.4 \mathrm{MHz}, \mathrm{CDCl}_{3}\right) \delta-109.9$ (ddd, $\left.J_{1}=8.9 J_{2}=6.4 J_{3}=1.4\right)$. ${ }^{13} \mathrm{C}$ NMR (75.5 MHz, $\left.\mathrm{CDCl}_{3}\right) \delta 43.0\left(\mathrm{CH}_{2}\right), 71.2(\mathrm{CH}), 88.9(\mathrm{C}), 92.1\left(\mathrm{t}, J_{\mathrm{CF}}=3.9, \mathrm{C}\right)$, $104.6\left(\mathrm{t}, J_{\mathrm{CF}}=25.3, \mathrm{CH}\right), 114.3(\mathrm{~d}, J=17.6, \mathrm{CH}), 114.4(\mathrm{~d}, J=17.8, \mathrm{CH}), 118.5\left(\mathrm{CH}_{2}\right)$, $119.5(\mathrm{C}), 125.4(\mathrm{CH}), 125.7\left(\mathrm{t}, J_{\mathrm{CF}}=11.7, \mathrm{C}\right), 127.2(\mathrm{CH}), 129.4(\mathrm{CH}), 132.3(\mathrm{CH})$, $134.4(\mathrm{CH}), 146.0(\mathrm{C}), 162.7\left(\mathrm{~d}, J_{\mathrm{CF}}=250.0, \mathrm{C}\right), 162.8$ (d, $\left.J_{\mathrm{CF}}=249.5, \mathrm{C}\right)$. HRMS (EI) calcd for $\mathrm{C}_{18} \mathrm{H}_{14} \mathrm{~F}_{2} \mathrm{O}[\mathrm{M}-\mathrm{OH}]^{+}: 267.0980$, found: 267.0979. The enantiomeric excess was determined with the aid of HPLC analysis: Chiralcel OD-H $(25 \mathrm{~cm} \times 0.46 \mathrm{~cm}$ column), hexane:isopropanol 99:1 as eluent and flow $=0.8 \mathrm{~mL} / \mathrm{min}: t_{\mathrm{R}}$ minor $=42.7 \mathrm{~min}$, $t_{\mathrm{R} \text { major }}=44.2 \mathrm{~min}$.

(R)-1-(4-Methyl-2-(phenylethynyl)phenyl)but-3-en-1-ol (3l):<smiles>C=CCC(O)c1ccc(C)cc1C#Cc1ccccc1</smiles>

Flash chromatography of the crude reaction product [n-hexane:EtOAc (10:1)] gave a white solid (optimized conditions: $79 \%, 96 \%$ ee). $[\alpha]_{\mathrm{D}}^{25}=+93.8$ (c 1.0; $\mathrm{CHCl}_{3}$ ). mp 70$72{ }^{\circ} \mathrm{C} .{ }^{1} \mathrm{H}$ NMR $\left(300 \mathrm{MHz}, \mathrm{CDCl}_{3}\right) \delta 2.25$ (br s, OH), 2.35 (s, 3H), 2.45-56 (m, 1H), 2.67-2.76 (m, 1H), 5.14-5.23 (m, 2H), $5.27\left(\mathrm{dd}, J_{1}=8.3 J_{2}=4.3,1 \mathrm{H}\right), 5.85-5.93(\mathrm{~m}, 1 \mathrm{H})$, 7.17-7.20 (m, 1H), 7.34-7.39 (m, 4H), 7.44 (d, J=8.0, 1H), 7.50-7.54 (m, 2H). ${ }^{13} \mathrm{C}$ NMR (75.5 MHz, $\left.\mathrm{CDCl}_{3}\right) \delta 20.8\left(\mathrm{CH}_{3}\right), 42.9\left(\mathrm{CH}_{2}\right), 71.3(\mathrm{CH}), 87.2(\mathrm{C}), 94.1(\mathrm{C}), 118.1$ 
$\left(\mathrm{CH}_{2}\right), 120.2(\mathrm{C}), 123.2(\mathrm{C}), 125.2(\mathrm{CH}), 128.4(\mathrm{CH}), 128.4(2 \times \mathrm{CH}), 129.6(\mathrm{CH}), 131.4$ (2xCH), $132.6(\mathrm{CH}), 134.8(\mathrm{CH}), 136.7(\mathrm{C}), 142.8(\mathrm{C})$. HRMS (EI) calcd for $\mathrm{C}_{19} \mathrm{H}_{18} \mathrm{O}$ $[\mathrm{M}-\mathrm{OH}]^{+}:$245.1325, found: 245.1325. The enantiomeric excess was determined as described in procedure $\mathrm{c}$, conditions $\mathrm{A}$ (page 5): $t_{\mathrm{R} \text { minor }}=5.7 \mathrm{~min}, t_{\mathrm{R} \text { major }}=6.7 \mathrm{~min}$.

(R)-3-(1-Hydroxybut-3-en-1-yl)-4-(phenylethynyl)phenol (3m):<smiles>C=CCC(O)c1cc(O)ccc1C#Cc1ccccc1</smiles>

Flash chromatography of the crude reaction product [ $n$-hexane:EtOAc (5:1)] gave a white solid (optimized conditions: $99 \%, 81 \%$ ee). $[\alpha] \mathrm{D}^{25}=+73.8$ (c 1.0; $\mathrm{CHCl}_{3}$ ). mp 84$86{ }^{\circ} \mathrm{C} .{ }^{1} \mathrm{H}$ NMR $\left(300 \mathrm{MHz}, \mathrm{CDCl}_{3}\right) \delta$ 2.41-2.51 (m, 1H), 2.68-2.76 (m, 1H), 2.74 (br s, $\mathrm{OH}), 5.14-5.22(\mathrm{~m}, 2 \mathrm{H}), 5.28\left(\mathrm{dd}, J_{1}=8.3 J_{2}=4.0,1 \mathrm{H}\right), 5.82-5.96(\mathrm{~m}, 1 \mathrm{H}), 6.43(\mathrm{br} \mathrm{s}$, $\mathrm{OH}), 6.73\left(\mathrm{dd}, J_{1}=8.3 J_{2}=2.6,1 \mathrm{H}\right), 7.08(\mathrm{~d}, J=2.6,1 \mathrm{H}), 7.33-7.40(\mathrm{~m}, 4 \mathrm{H}), 7.45-7.50$ $(\mathrm{m}, 2 \mathrm{H}) .{ }^{13} \mathrm{C} \mathrm{NMR}\left(75.5 \mathrm{MHz}, \mathrm{CDCl}_{3}\right) \delta 42.5\left(\mathrm{CH}_{2}\right), 71.6(\mathrm{CH}), 86.9(\mathrm{C}), 93.2(\mathrm{C})$, $112.4(\mathrm{CH}), 112.4(\mathrm{C}), 114.7(\mathrm{CH}), 118.6\left(\mathrm{CH}_{2}\right), 123.3(\mathrm{C}), 128.1(\mathrm{CH}), 128.4(2 x \mathrm{CH})$, $131.2(2 \mathrm{xCH}), 133.9(\mathrm{CH}), 134.3(\mathrm{CH}), 147.3(\mathrm{C}), 156.4(\mathrm{C})$. HRMS (EI) calcd for $\mathrm{C}_{18} \mathrm{H}_{16} \mathrm{O}_{2} \quad[\mathrm{M}-\mathrm{OH}]^{+}:$247.1117, found: 247.1124. The enantiomeric excess was determined with the aid of HPLC analysis: Chiralpak IC (25 cm x $0.46 \mathrm{~cm}$ column), hexane:isopropanol 95:5 as eluent and flow $=1 \mathrm{~mL} / \mathrm{min}: t_{\mathrm{R}}$ minor $=19.3 \mathrm{~min}, t_{\mathrm{R} \text { major }}=$ $16.6 \mathrm{~min}$.

(R)-1-(6-(Phenylethynyl)benzo[d][1,3]dioxol-5-yl)but-3-en-1-ol (3n):<smiles>C=CCC(O)c1cc2c(cc1C#Cc1ccccc1)OCO2</smiles>

Flash chromatography of the crude reaction product [ $n$-hexane:EtOAc $(10: 1)]$ gave a white solid (optimized conditions: $73 \%, 70 \%$ ee). $[\alpha]_{\mathrm{D}}{ }^{25}=+95.5\left(\right.$ c $\left.1.0 ; \mathrm{CHCl}_{3}\right)$. mp 83$85{ }^{\circ} \mathrm{C} .{ }^{1} \mathrm{H}$ NMR $\left(300 \mathrm{MHz}, \mathrm{CDCl}_{3}\right) \delta 2.18(\mathrm{~d}, \mathrm{~J}=2.5, \mathrm{OH}), 2.40-2.51(\mathrm{~m}, 1 \mathrm{H}), 2.62-2.71$ (m, 1H), 5.14-5.23 (m, 2H), 5.25-5.28 (m, 1H), 5.83-5.97 (m, 1H), $5.99(\mathrm{~s}, 2 \mathrm{H}), 6.94(\mathrm{~s}$, 1H), $7.06(\mathrm{~s}, 1 \mathrm{H}), 7.33-7.39(\mathrm{~m}, 3 \mathrm{H}), 7.46-7.50(\mathrm{~m}, 2 \mathrm{H}) .{ }^{13} \mathrm{C} \mathrm{NMR}\left(75.5 \mathrm{MHz}, \mathrm{CDCl}_{3}\right)$ 
$\delta 43.0\left(\mathrm{CH}_{2}\right), 71.1(\mathrm{CH}), 87.0(\mathrm{C}), 93.1(\mathrm{C}), 101.4\left(\mathrm{CH}_{2}\right), 106.1(\mathrm{CH}), 111.3(\mathrm{CH})$, $113.4(\mathrm{C}), 118.3\left(\mathrm{CH}_{2}\right), 123.2(\mathrm{C}), 128.2(\mathrm{CH}), 128.4(2 \mathrm{xCH}), 131.3(2 \mathrm{xCH}), 134.6$ $(\mathrm{CH}), 141.3$ (C), 146.4 (C), 148.4 (C). HRMS (EI) calcd for $\mathrm{C}_{19} \mathrm{H}_{16} \mathrm{O}_{3}[\mathrm{M}-\mathrm{OH}]^{+}$: 275.1067, found: 275.1068. The enantiomeric excess was determined as described in procedure $\mathrm{c}$, conditions $\mathrm{A}$ (page 5): $t_{\mathrm{R} \text { minor }}=63.7 \mathrm{~min}, t_{\mathrm{R} \text { major }}=68.9 \mathrm{~min}$.

(R)-1-(4-Fluoro-2-(phenylethynyl)phenyl)but-3-en-1-ol (3o):<smiles>C=CCC(O)c1ccc(F)cc1C#Cc1ccccc1</smiles>

Flash chromatography of the crude reaction product [n-hexane:EtOAc (10:1)] gave a white solid (optimized conditions: $99 \%, 97 \%$ ee). $[\alpha]_{\mathrm{D}}^{25}=+100.3$ (c 1.0; $\mathrm{CHCl}_{3}$ ). mp 89-91 ${ }^{\circ} \mathrm{C} .{ }^{1} \mathrm{H}$ NMR (300 MHz, $\left.\mathrm{CDCl}_{3}\right) \delta 2.27$ (br s, OH), 2.42-2.52 (m, 1H), 2.66-2.75 (m, 1H), $5.16(\mathrm{~m}, 1 \mathrm{H}), 5.19-5.23(\mathrm{~m}, 1 \mathrm{H}), 5.25-5.29$ (m, 1H), 5.83-5.91 (m, 1H), 7.04$7.10(\mathrm{~m}, 1 \mathrm{H}), 7.21\left(\mathrm{dd}, J_{1}=9.2 J_{2}=2.7,1 \mathrm{H}\right), 7.36-7.39(\mathrm{~m}, 3 \mathrm{H}), 7.49-7.55(\mathrm{~m}, 3 \mathrm{H}) .{ }^{19} \mathrm{~F}$ NMR $\left(282.4 \mathrm{MHz}, \mathrm{CDCl}_{3}\right) \delta-116.0\left(\mathrm{ddd}, J_{1}=J_{2}=8.7 J_{3}=5.8\right) .{ }^{13} \mathrm{C}$ NMR $(75.5 \mathrm{MHz}$, $\left.\mathrm{CDCl}_{3}\right) \delta 42.9\left(\mathrm{CH}_{2}\right), 70.8(\mathrm{CH}), 85.8\left(\mathrm{~d}, J_{\mathrm{CF}}=3.3, \mathrm{C}\right), 95.3(\mathrm{C}), 115.9\left(\mathrm{~d}, J_{\mathrm{CF}}=21.1\right.$, $\mathrm{CH}), 118.5\left(\mathrm{CH}_{2}\right), 118.5\left(\mathrm{~d}, J_{\mathrm{CF}}=22.9, \mathrm{CH}\right), 122.1\left(\mathrm{~d}, J_{\mathrm{CF}}=9.7, \mathrm{C}\right), 122.6(\mathrm{C}), 127.1(\mathrm{~d}$, $\left.J_{\mathrm{CF}}=8.7, \mathrm{CH}\right), 128.5(2 \mathrm{xCH}), 128.8(\mathrm{CH}), 131.5(2 \mathrm{xCH}), 134.4(\mathrm{CH}), 141.6\left(\mathrm{~d}, J_{\mathrm{CF}}=3.2\right.$, C), $161.3\left(\mathrm{~d}, J_{\mathrm{CF}}=245.9, \mathrm{C}\right)$. HRMS (EI) calcd for $\mathrm{C}_{18} \mathrm{H}_{15} \mathrm{FO}[\mathrm{M}-\mathrm{OH}]^{+}: 249.1074$, found: 249.1075. The enantiomeric excess was determined as described in procedure $\mathrm{c}$, conditions A (page 5): $t_{\mathrm{R} \text { minor }}=13.4 \mathrm{~min}, t_{\mathrm{R} \text { major }}=19.0 \mathrm{~min}$.

(R)-1-(2-(Phenylethynyl)-5-(trifluoromethyl)phenyl)but-3-en-1-ol (3p):<smiles>C=CC[C@H](O)c1cc(C(F)(F)F)ccc1C#Cc1ccccc1</smiles>

Flash chromatography of the crude reaction product [n-hexane:EtOAc (10:1)] gave a yellowish solid (optimized conditions: $50 \%, 95 \%$ ee). $[\alpha]_{\mathrm{D}}{ }^{25}=+85.3$ (c 1.0; $\mathrm{CHCl}_{3}$ ). mp $72-74{ }^{\circ} \mathrm{C} .{ }^{1} \mathrm{H}$ NMR $\left(300 \mathrm{MHz}, \mathrm{CDCl}_{3}\right) \delta 2.23$ (br s, OH), 2.40-2.51 (m, 1H), 2.72-2.81 $(\mathrm{m}, 1 \mathrm{H}), 5.19-5.26(\mathrm{~m}, 2 \mathrm{H}), 5.31-5.35(\mathrm{~m}, 1 \mathrm{H}), 5.85-5.98(\mathrm{~m}, 1 \mathrm{H}), 7.37-7.41(\mathrm{~m}, 3 \mathrm{H})$, 
7.50-7.55 (m, 3H), 7.60-7.63 (m, 1H), 7.87-7.88 (m, 1H). ${ }^{19} \mathrm{~F}$ NMR (282.4 MHz, $\left.\mathrm{CDCl}_{3}\right) \delta-63.2(\mathrm{~s}) .{ }^{13} \mathrm{C} \mathrm{NMR}\left(75.5 \mathrm{MHz}, \mathrm{CDCl}_{3}\right) \delta 42.8\left(\mathrm{CH}_{2}\right), 70.8(\mathrm{CH}), 85.7(\mathrm{C})$, $96.8(\mathrm{C}), 119.0\left(\mathrm{CH}_{2}\right), 122.4\left(\mathrm{q}, J_{\mathrm{CF}}=4.1, \mathrm{CH}\right), 122.4(\mathrm{C}), 123.8\left(\mathrm{q}, J_{\mathrm{CF}}=3.7, \mathrm{CH}\right), 124.0$ $\left(\mathrm{q}, J_{\mathrm{CF}}=272.2, \mathrm{CF}_{3}\right), 124.1\left(\mathrm{q}, J_{\mathrm{CF}}=1.3, \mathrm{C}\right), 128.6(2 \mathrm{xCH}), 129.0(\mathrm{CH}), 130.3(\mathrm{q}$, $\left.J_{\mathrm{CF}}=33.1, \mathrm{C}\right), 131.6(2 \mathrm{xCH}), 132.4(\mathrm{CH}), 134.1(\mathrm{CH}), 146.5(\mathrm{C})$. HRMS (EI) calcd for $\mathrm{C}_{19} \mathrm{H}_{15} \mathrm{~F}_{3} \mathrm{O} \quad[\mathrm{M}-\mathrm{OH}]^{+}:$299.1042, found: 299.1046. The enantiomeric excess was determined as described in procedure c, conditions $A($ page 5$): t_{\mathrm{R} \text { minor }}=8.7 \mathrm{~min}, t_{\mathrm{R} \text { major }}$ $=12.7 \mathrm{~min}$.

\section{d) Propargylation:}

Table S1. Influence of the substitution at the triple bond on the $(R)$-TRIP-PA catalyzed propargylation of ortho-alkynylbenzaldehydes:<smiles>[R]C#Cc1ccccc1C=O</smiles><smiles>[R]C#Cc1ccccc1C(O)CC#C</smiles>

\begin{tabular}{cccc}
\hline Entry & $\mathrm{R}$ & Yield (\%) & ee (\%) \\
\hline 1 & $\mathrm{H}$ & 75 & 25 \\
2 & $\mathrm{Bu}$ & 70 & 28 \\
3 & $\mathrm{Cy}$ & 81 & 51 \\
4 & $\mathrm{Ph}$ & 99 & 88 \\
\hline
\end{tabular}

Table S2. Optimization for $\mathrm{R}=\mathrm{Ph}$ :<smiles>O=Cc1ccccc1C#Cc1ccccc1</smiles>

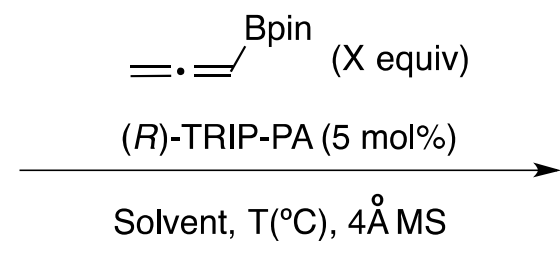<smiles>C#CCC(O)c1ccccc1C#Cc1ccccc1</smiles>

\begin{tabular}{cccccc}
\hline Entry & $\mathrm{X}$ & Solvent & Temp $\left({ }^{\circ} \mathrm{C}\right)$ & Yield (\%) & ee (\%) \\
\hline 1 & 2 & Cyclohexane & o & 99 & 88 \\
2 & 2 & Toluene & -30 & 81 & 91 \\
3 & 2 & DCM & -30 & 70 & 66 \\
4 & 2 & Toluene & -78 & 60 & 80 \\
5 & 1.2 & Toluene & -30 & 64 & 94 \\
6 & 3 & Toluene & -30 & 73 & 87 \\
\hline
\end{tabular}


General procedure for the asymmetric propargylation:

A solution of the corresponding 2-alkynylbenzaldehyde $\mathbf{1 d}, \mathbf{j}, \mathbf{0 , p}$ (typically $0.1 \mathrm{mmol}$ ) in dry toluene $(0.1 \mathrm{M})$ was treated with activated $4 \AA \mathrm{MS}(50 \mathrm{mg} / 0.1 \mathrm{mmol}$ of $\mathbf{1})$ for 5 minutes. Then $(R)$-TRIP-PA (5 mol\%) was added and the reaction mixture was cooled to $-30{ }^{\circ} \mathrm{C}$ and allenylboronic acid pinacol ester 4 (1.2 equiv) was added dropwise. Upon completion (approximately $1 \mathrm{~h}$ ), solvents were removed under reduced pressure and the residue purified by flash chromatography using mixtures of $n$-hexanes:ethyl acetate as eluent.

(R)-1-(2-(Phenylethynyl)phenyl)but-3-yn-1-ol (5d):<smiles>C#CCC(O)c1ccccc1C#Cc1ccccc1</smiles>

Flash chromatography of the crude reaction product [n-hexane:EtOAc (10:1)] gave a white solid $(64 \%, 94 \%$ ee $) . \mathrm{mp} 78-80{ }^{\circ} \mathrm{C} .[\alpha]_{\mathrm{D}}{ }^{25}=+62.9\left(\mathrm{c} 1.0 ; \mathrm{CHCl}_{3}\right) .{ }^{1} \mathrm{H}$ NMR $(300$ $\left.\mathrm{MHz} \mathrm{CDCl}_{3}\right) \delta 2.11(\mathrm{t}, J=2.6,1 \mathrm{H}), 2.61(\mathrm{br} \mathrm{s}, \mathrm{OH}), 2.65\left(\mathrm{ddd}, J_{1}=16.8 J_{2}=8.0 J_{3}=2.6\right.$, $1 \mathrm{H}), 2.93\left(\mathrm{ddd}, J_{1}=16.8 J_{2}=4.1 J_{3}=2.7,1 \mathrm{H}\right), 5.43\left(\mathrm{dd}, J_{1}=8.0 J_{2}=4.1,1 \mathrm{H}\right), 7.29(\mathrm{td}$, $\left.J_{1}=7.6 J_{2}=1.7,1 \mathrm{H}\right), 7.35-7.42(\mathrm{~m}, 4 \mathrm{H}), 7.51-7.57(\mathrm{~m}, 3 \mathrm{H}), 7.61-7.64(\mathrm{~m}, 1 \mathrm{H}) .{ }^{13} \mathrm{C} \mathrm{NMR}$ $\left(75.5 \mathrm{MHz}, \mathrm{CDCl}_{3}\right) \delta 28.4\left(\mathrm{CH}_{2}\right), 70.5(\mathrm{CH}), 70.9(\mathrm{CH}), 80.8(\mathrm{C}), 86.6(\mathrm{C}), 95.0(\mathrm{C})$, $120.5(\mathrm{C}), 122.9(\mathrm{C}), 125.2(\mathrm{CH}), 127.5(\mathrm{CH}), 128.4(2 \times \mathrm{CH}), 128.6(\mathrm{CH}), 128.7(\mathrm{CH})$, $131.5(2 \times C H), 132.1(\mathrm{CH}), 144.1(\mathrm{C})$. HRMS (EI) calcd for $\mathrm{C}_{18} \mathrm{H}_{14} \mathrm{O}[\mathrm{M}]^{+}:$: 246.1045, found: 246.1065. The enantiomeric excess was determined with the aid of HPLC analysis: Chiralpak IC (25 cm x $0.46 \mathrm{~cm}$ column), hexane:isopropanol 99:1 as eluent and flow $=0.8 \mathrm{~mL} / \mathrm{min}: t_{\mathrm{R} \text { minor }}=42.7 \mathrm{~min}, t_{\mathrm{R} \text { major }}=48.8 \mathrm{~min}$.

\section{(R)-1-(2-((2-Nitrophenyl)ethynyl)phenyl)but-3-yn-1-ol (5j):}<smiles>C#CC[C@H](O)c1ccccc1C#Cc1ccccc1[N+](=O)[O-]</smiles>

Flash chromatography of the crude reaction product [ $n$-hexane:EtOAc (5:1)] gave a yellow oil $\left(62 \%, 85 \%\right.$ ee). $[\alpha]_{\mathrm{D}}{ }^{25}=+43.0\left(\mathrm{c} 1.0 ; \mathrm{CHCl}_{3}\right) .{ }^{1} \mathrm{H} \mathrm{NMR}\left(300 \mathrm{MHz}, \mathrm{CDCl}_{3}\right) \delta$ $2.09(\mathrm{t}, J=2.6,1 \mathrm{H}), 2.67(\mathrm{br} \mathrm{s}, \mathrm{OH}), 2.70\left(\mathrm{ddd}, J_{1}=16.8 J_{2}=7.4 J_{3}=2.6,1 \mathrm{H}\right), 2.95$ (ddd, 
$\left.J_{1}=16.9 J_{2}=4.5 J_{3}=2.6,1 \mathrm{H}\right), 5.47-5.51(\mathrm{~m}, 1 \mathrm{H}), 7.32\left(\mathrm{td}, J_{1}=7.5 J_{2}=1.3,1 \mathrm{H}\right), 7.42-7.52$ $(\mathrm{m}, 2 \mathrm{H}), 7.58-7.67(\mathrm{~m}, 3 \mathrm{H}), 7.73-7.76(\mathrm{~m}, 1 \mathrm{H}), 8.12\left(\mathrm{ddd}, J_{1}=J_{2}=8.2 J_{3}=1.3,1 \mathrm{H}\right) .{ }^{13} \mathrm{C}$ NMR (75.5 MHz, $\left.\mathrm{CDCl}_{3}\right) \delta 28.5\left(\mathrm{CH}_{2}\right), 70.0(\mathrm{CH}), 71.1(\mathrm{CH}), 80.5(\mathrm{C}), 89.9(\mathrm{C}), 94.5$ (C), $118.6(\mathrm{C}), 119.7(\mathrm{C}), 124.9(\mathrm{CH}), 125.6(\mathrm{CH}), 127.6(\mathrm{CH}), 128.8(\mathrm{CH}), 129.7$ $(\mathrm{CH}), 132.9(\mathrm{CH}), 133.0(\mathrm{CH}), 134.8(\mathrm{CH}), 144.8(\mathrm{C}), 149.1(\mathrm{C})$. HRMS (EI) calcd for $\mathrm{C}_{18} \mathrm{H}_{13} \mathrm{NO}_{3}[\mathrm{M}-\mathrm{OH}]^{+}:$274.0863, found: 274.0857. The enantiomeric excess was determined with the aid of HPLC analysis: Chiralcel OD-H (25 cm x $0.46 \mathrm{~cm}$ column), hexane:isopropanol 95:5 as eluent and flow $=1 \mathrm{~mL} / \mathrm{min}: t_{\mathrm{R} \text { minor }}=40.2 \mathrm{~min}, t_{\mathrm{R} \text { major }}=$ $38.2 \mathrm{~min}$.

(R)-1-(4-Fluoro-2-(phenylethynyl)phenyl)but-3-yn-1-ol (5o):<smiles>C#CCC(O)c1ccc(F)cc1C#Cc1ccccc1</smiles>

Flash chromatography of the crude reaction product [n-hexane:EtOAc (10:1)] gave a white solid $(51 \%, 90 \%$ ee $)$. $[\alpha]_{\mathrm{D}}{ }^{25}=+65.6$ (c 1.0; $\left.\mathrm{CHCl}_{3}\right)$. mp 95-97 ${ }^{\circ} \mathrm{C} .{ }^{1} \mathrm{H}$ NMR $(300$ $\mathrm{MHz}_{\mathrm{CDCl}} \mathrm{CD} \delta 2.11(\mathrm{t}, J=2.6,1 \mathrm{H}), 2.62\left(\mathrm{ddd}, J_{1}=16.8 J_{2}=7.9 J_{3}=2.6,1 \mathrm{H}\right), 2.89(\mathrm{ddd}$, $\left.J_{1}=16.8 J_{2}=4.2 J_{3}=2.6,1 \mathrm{H}\right), 5.39\left(\mathrm{dd}, J_{1}=7.9 J_{2}=4.2,1 \mathrm{H}\right), 7.08(\mathrm{~m}, 1 \mathrm{H}), 7.21(\mathrm{~m}, 1 \mathrm{H})$, 7.36-7.40 (m, 3H), 7.53-7.61 (m, 3H). ${ }^{19} \mathrm{~F}$ NMR (282.4 MHz, $\left.\mathrm{CDCl}_{3}\right) \delta-115.3(\mathrm{td}$, $\left.J_{1}=8.7 J_{2}=5.7\right) .{ }^{13} \mathrm{C}$ NMR $\left(75.5 \mathrm{MHz}, \mathrm{CDCl}_{3}\right) \delta 28.5\left(\mathrm{CH}_{2}\right), 69.9(\mathrm{CH}), 71.1(\mathrm{CH}), 80.5$ $(\mathrm{C}), 85.4\left(\mathrm{~d}, J_{\mathrm{CF}}=3.2, \mathrm{C}\right), 95.8(\mathrm{C}), 115.9\left(\mathrm{~d}, J_{\mathrm{CF}}=21.4, \mathrm{CH}\right), 118.5\left(\mathrm{~d}, J_{\mathrm{CF}}=23.0, \mathrm{CH}\right)$, $122.2\left(\mathrm{~d}, J_{\mathrm{CF}}=9.6, \mathrm{C}\right), 122.4(\mathrm{C}), 127.1\left(\mathrm{~d}, J_{\mathrm{CF}}=8.7, \mathrm{CH}\right), 128.5(2 \mathrm{xCH}), 128.9(\mathrm{CH})$, $131.6(2 \mathrm{xCH}), 140.0\left(\mathrm{~d}, J_{\mathrm{CF}}=3.3, \mathrm{C}\right), 161.6\left(\mathrm{~d}, J_{\mathrm{CF}}=246.8, \mathrm{C}\right)$. HRMS (EI) calcd for $\mathrm{C}_{18} \mathrm{H}_{13} \mathrm{FO}[\mathrm{M}-\mathrm{OH}]^{+}:$247.0918, found: 247.0922. The enantiomeric excess was determined with the aid of HPLC analysis: Chiralcel OD-H (25 cm x $0.46 \mathrm{~cm}$ column), hexane:isopropanol 98:2 as eluent and flow $=1 \mathrm{~mL} / \mathrm{min}: t_{\mathrm{R} \text { minor }}=25.0 \mathrm{~min}, t_{\mathrm{R} \text { major }}=$ $33.5 \mathrm{~min}$.

(R)-1-(2-(Phenylethynyl)-5-(trifluoromethyl)phenyl)but-3-yn-1-ol (5p):<smiles>C#CCC(O)c1cc(C(F)(F)F)ccc1C#Cc1ccccc1</smiles> 
Flash chromatography of the crude reaction product [n-hexane:EtOAc (10:1)] gave a white solid $(56 \%, 93 \%$ ee $) .[\alpha]_{\mathrm{D}}{ }^{25}=+59.7$ (c 1.0; $\left.\mathrm{CHCl}_{3}\right) . \mathrm{mp} 105-107{ }^{\circ} \mathrm{C} .{ }^{1} \mathrm{H}$ NMR $\left(300 \mathrm{MHz}, \mathrm{CDCl}_{3}\right) \delta 2.14(\mathrm{t}, J=2.6,1 \mathrm{H}), 2.62\left(\mathrm{ddd}, J_{1}=16.8 J_{2}=7.9 J_{3}=2.6,1 \mathrm{H}\right), 2.67(\mathrm{br}$ s, OH), $2.95\left(\mathrm{ddd}, J_{1}=16.8 J_{2}=3.9 J_{3}=2.6,1 \mathrm{H}\right), 5.45\left(\mathrm{dd}, J_{1}=7.9 J_{2}=3.9,1 \mathrm{H}\right), 7.38-7.42$ $(\mathrm{m}, 3 \mathrm{H}), 7.52-7.63(\mathrm{~m}, 4 \mathrm{H}), 7.92-7.93(\mathrm{~m}, 1 \mathrm{H}) .{ }^{19} \mathrm{~F}$ NMR $\left(282.4 \mathrm{MHz}, \mathrm{CDCl}_{3}\right) \delta-63.2$ (s). ${ }^{13} \mathrm{C}$ NMR (75.5 MHz, $\left.\mathrm{CDCl}_{3}\right) \delta 28.3\left(\mathrm{CH}_{2}\right), 69.9(\mathrm{CH}), 71.5(\mathrm{CH}), 80.1(\mathrm{C}), 85.3$ (C), $97.3(\mathrm{C}), 122.2(\mathrm{C}), 122.4$ (q, $\left.J_{\mathrm{CF}}=3.7, \mathrm{CH}\right), 123.9$ (q, $J_{\mathrm{CF}}=272.2, \mathrm{CF}_{3}$ ), 124.1 (q, $\left.J_{\mathrm{CF}}=1.3, \mathrm{C}\right), 124.3\left(\mathrm{q}, J_{\mathrm{CF}}=3.8, \mathrm{CH}\right), 128.6(2 \times \mathrm{CH}), 129.2(\mathrm{CH}), 130.3\left(\mathrm{q}, J_{\mathrm{CF}}=32.8, \mathrm{C}\right)$, $131.7(2 \times C H), 132.3(\mathrm{CH}), 144.8(\mathrm{C})$. HRMS (EI) calcd for $\mathrm{C}_{19} \mathrm{H}_{13} \mathrm{~F}_{3} \mathrm{O}[\mathrm{M}-\mathrm{OH}]^{+}$: 297.0886, found: 297.0884. The enantiomeric excess was determined with the aid of HPLC analysis: Chiralcel OD-H (25 cm x $0.46 \mathrm{~cm}$ column), hexane:isopropanol 98:2 as eluent and flow $=1 \mathrm{~mL} / \mathrm{min}: t_{\mathrm{R} \text { minor }}=15.4 \mathrm{~min}, t_{\mathrm{R} \text { major }}=25.8 \mathrm{~min}$.

\section{e) General procedure for the RCEYM process:}

To a solution of the corresponding enyne 3d,o in dry DCM $(0.1 \mathrm{M})$ in a sealed tube, $2^{\text {nd }}$ generation Grubbs' catalyst (10 mol\%) and 1,7-octadiene (4 equiv.) were added and the reaction mixture was heated at $45^{\circ} \mathrm{C}$ overnight. Upon completion, the reaction mixture was allowed to reach room temperature, solvents were removed under reduced pressure and the residue purified by flash chromatography using mixtures of $n$-hexanes:ethyl acetate as eluent.

\section{(R)-4-(1-Phenylvinyl)-1,2-dihydronaphthalen-1-ol (6d):}<smiles>C=C(C1=CC[C@@H](O)c2ccccc21)c1ccccc1</smiles>

Flash chromatography of the crude reaction product [ $n$-hexane:EtOAc $(5: 1)]$ gave a yellow oil $\left(75 \%, 90 \%\right.$ ee). $[\alpha]_{\mathrm{D}}{ }^{25}=-52.3\left(\mathrm{c} 1.0 ; \mathrm{CHCl}_{3}\right) .{ }^{1} \mathrm{H} \mathrm{NMR}\left(300 \mathrm{MHz}, \mathrm{CDCl}_{3}\right) \delta$ 1.84 (br s, OH), $2.71\left(\mathrm{dd}, J_{1}=5.7 J_{2}=4.5,2 \mathrm{H}\right), 4.89(\mathrm{t}, J=5.7,1 \mathrm{H}), 5.38(\mathrm{~d}, J=1.7,1 \mathrm{H})$, $5.69(\mathrm{~d}, J=1.7,1 \mathrm{H}), 6.09(\mathrm{t}, J=4.4,1 \mathrm{H}), 7.02\left(\mathrm{dd}, J_{1}=7.6 J_{2}=1.4,1 \mathrm{H}\right), 7.13\left(\mathrm{td}, J_{1}=7.5\right.$ $\left.J_{2}=1.5,1 \mathrm{H}\right), 7.18-7.31(\mathrm{~m}, 4 \mathrm{H}), 7.40-7.46(\mathrm{~m}, 3 \mathrm{H}) .{ }^{13} \mathrm{C} \mathrm{NMR}\left(75.5 \mathrm{MHz}, \mathrm{CDCl}_{3}\right) \delta 32.6$ $\left(\mathrm{CH}_{2}\right), 68.0(\mathrm{CH}), 115.2\left(\mathrm{CH}_{2}\right), 125.1(\mathrm{CH}), 126.3(\mathrm{CH}), 126.5(\mathrm{CH}), 126.6(2 \times \mathrm{CH})$, $127.5(\mathrm{CH}), 127.7(\mathrm{CH}), 128.2(\mathrm{CH}), 128.4(2 \times \mathrm{CH}), 133.0(\mathrm{C}), 136.9(\mathrm{C}), 138.9(\mathrm{C})$, 139.7 (C), 148.0 (C). HRMS (EI) calcd for $\mathrm{C}_{18} \mathrm{H}_{16} \mathrm{O}[\mathrm{M}-\mathrm{OH}]^{+}$: 231.1168, found: 
231.1159. The enantiomeric excess was determined with the aid of HPLC analysis: Chiralpak IC (25 cm x 0.46 cm column), hexane:isopropanol 95:5 as eluent and flow = $1 \mathrm{~mL} / \mathrm{min}: t_{\mathrm{R} \text { minor }}=7.8 \mathrm{~min}, t_{\mathrm{R} \text { major }}=17.6 \mathrm{~min}$.

\section{(R)-6-Fluoro-4-(1-phenylvinyl)-1,2-dihydronaphthalen-1-ol (6o):}<smiles>C=C(C1=CC[C@@H](O)c2ccc(F)cc21)c1ccccc1</smiles>

Flash chromatography of the crude reaction product [ $n$-hexane:EtOAc (20:1)] gave a colorless oil $(71 \%, 91 \%$ ee $) .[\alpha]_{\mathrm{D}}{ }^{25}=-22.4\left(\mathrm{c} 1.0 ; \mathrm{CHCl}_{3}\right) .{ }^{1} \mathrm{H}$ NMR $\left(300 \mathrm{MHz}, \mathrm{CDCl}_{3}\right)$ $\delta 1.78($ br s, OH), $2.61(\mathrm{~m}, 2 \mathrm{H}), 4.76(\mathrm{t}, J=5.7,1 \mathrm{H}), 5.28(\mathrm{~d}, J=1.5,1 \mathrm{H}), 5.61(\mathrm{~d}, J=1.6$, $1 \mathrm{H}), 6.04(\mathrm{t}, J=4.4,1 \mathrm{H}), 6.64\left(\mathrm{dd}, J_{1}=10.1 J_{2}=2.7,1 \mathrm{H}\right), 6.78\left(\mathrm{td}, J_{1}=8.4 J_{2}=2.6,1 \mathrm{H}\right)$, 7.16-7.36 (m, 6H). ${ }^{19} \mathrm{~F}$ NMR $\left(282.4 \mathrm{MHz}, \mathrm{CDCl}_{3}\right) \delta-113.6\left(\mathrm{ddd}, J_{1}=J_{2}=9.2 J_{3}=5.8\right) .{ }^{13} \mathrm{C}$ NMR (75.5 MHz, $\left.\mathrm{CDCl}_{3}\right) \delta 32.7\left(\mathrm{CH}_{2}\right), 67.3(\mathrm{CH}), 113.2\left(\mathrm{~d}, J_{\mathrm{CF}}=23.0, \mathrm{CH}\right), 113.8(\mathrm{~d}$, $\left.J_{\mathrm{CF}}=21.7, \mathrm{CH}\right), 115.6\left(\mathrm{CH}_{2}\right), 126.4(\mathrm{CH}), 126.5(2 \mathrm{xCH}), 127.9(\mathrm{CH}), 128.2\left(\mathrm{~d}, J_{\mathrm{CF}}=8.4\right.$, $\mathrm{CH}), 128.5(2 \times \mathrm{CH}), 132.7\left(\mathrm{~d}, J_{\mathrm{CF}}=3.0, \mathrm{C}\right), 135.2\left(\mathrm{~d}, J_{\mathrm{CF}}=8.1, \mathrm{C}\right), 138.3\left(\mathrm{~d}, J_{\mathrm{CF}}=2.2, \mathrm{C}\right)$, 139.4 (C), 147.5 (C), 162.7 (d, $J_{\mathrm{CF}}=245.1, \mathrm{C}$ ). HRMS (EI) calcd for $\mathrm{C}_{18} \mathrm{H}_{15} \mathrm{FO}$ [M$\mathrm{OH}]^{+}: 249.1074$, found: 249.1068 . The enantiomeric excess was determined with the aid of HPLC analysis: Chiralpak IC (25 cm x $0.46 \mathrm{~cm}$ column), hexane:isopropanol $90: 10$ as eluent and flow $=1 \mathrm{~mL} / \mathrm{min}: t_{\mathrm{R} \text { minor }}=4.8 \mathrm{~min}, t_{\mathrm{R} \text { major }}=7.2 \mathrm{~min}$.

f) General procedure for the protection with TBS:

To a solution of the corresponding enyne 3d,l in DMF (0.2M), TBSCl (2 equiv.) and imidazole (2.5 equiv.) were added and the reaction mixture was stirred at room temperature. When the starting material was consumed (approximately 6 hours), the reaction mixture was quenched with water and extracted with AcOEt. The organic layers were combined, dried over $\mathrm{Na}_{2} \mathrm{SO}_{4}$, filtered and evaporated under reduced pressure. The crude product was purified by flash chromatography employing mixtures of $n$-hexane:ethyl acetate as eluents. It was assumed that the enantiomeric excess was preserved and was therefore not determined. 
(R)-tert-Butyldimethyl(1-(2-(phenylethynyl)phenyl)but-3-enyloxy)silane (7d):

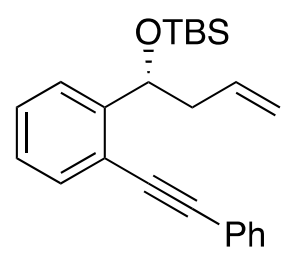

Flash chromatography of the crude reaction product [ $n$-hexane:EtOAc (100:1)] gave a colorless oil $(81 \%) .[\alpha]_{\mathrm{D}}{ }^{25}=+39.4\left(\mathrm{c} 1.0 ; \mathrm{CHCl}_{3}\right) .{ }^{1} \mathrm{H}$ NMR $\left(300 \mathrm{MHz}, \mathrm{CDCl}_{3}\right) \delta-0.09$ $(\mathrm{s}, 3 \mathrm{H}), 0.06(\mathrm{~s}, 3 \mathrm{H}), 0.90(\mathrm{~s}, 9 \mathrm{H}), 2.41-2.58(\mathrm{~m}, 2 \mathrm{H}), 5.02(\mathrm{~m}, 1 \mathrm{H}), 5.05-5.08(\mathrm{~m}, 1 \mathrm{H})$, $5.32\left(\mathrm{dd}, J_{1}=7.6 J_{2}=4.4,1 \mathrm{H}\right), 5.86-5.96(\mathrm{~m}, 1 \mathrm{H}), 7.22\left(\mathrm{td}, J_{1}=7.5 J_{2}=1.4,1 \mathrm{H}\right), 7.32-7.41$ $(\mathrm{m}, 4 \mathrm{H}), 7.46-7.59(\mathrm{~m}, 4 \mathrm{H}) .{ }^{13} \mathrm{C} \mathrm{NMR}\left(75.5 \mathrm{MHz}, \mathrm{CDCl}_{3}\right) \delta-5.0\left(\mathrm{CH}_{3}\right),-4.7\left(\mathrm{CH}_{3}\right)$, $18.3(\mathrm{C}), 25.9\left(3 \mathrm{xCH}_{3}\right), 44.3\left(\mathrm{CH}_{2}\right), 72.6(\mathrm{CH}), 87.2(\mathrm{C}), 94.0(\mathrm{C}), 116.9\left(\mathrm{CH}_{2}\right), 119.9$ (C), $123.3(\mathrm{C}), 126.0(\mathrm{CH}), 126.6(\mathrm{CH}), 128.3(\mathrm{CH}), 128.4(2 \mathrm{xCH}), 128.4(\mathrm{CH}), 131.4$ $(2 \times C H), 131.6(\mathrm{CH}), 135.5(\mathrm{CH}), 147.1(\mathrm{C})$. HRMS (EI) calcd for $\mathrm{C}_{24} \mathrm{H}_{30} \mathrm{OSi}[\mathrm{M}]^{+}$: 362.2066, found: 362.2086 .

\section{(R)-tert-butyldimethyl((1-(4-methyl-2-(phenylethynyl)phenyl)but-3-en-1-} yl)oxy)silane (7l):<smiles>C=CCC([O+])c1ccc(C)cc1C#Cc1ccccc1</smiles>

Flash chromatography of the crude reaction product [n-hexane:EtOAc (100:1)] gave a colorless oil (50\%). $[\alpha]_{\mathrm{D}}{ }^{25}=+82.0\left(\mathrm{c} 1.0 ; \mathrm{CHCl}_{3}\right)$. ${ }^{1} \mathrm{H} \mathrm{NMR}\left(300 \mathrm{MHz}, \mathrm{CDCl}_{3}\right) \delta-0.09$ (s, 3H), -0.06 (s, 3H), $0.90(\mathrm{~s}, 9 \mathrm{H}), 2.34(\mathrm{~s}, 3 \mathrm{H}), 2.40-2.56(\mathrm{~m}, 2 \mathrm{H}), 5.00-5.01(\mathrm{~m}, 1 \mathrm{H})$, 5.04-5.08 (m, 1H), $5.28\left(\mathrm{dd}, J_{1}=7.4 J_{2}=4.6,1 \mathrm{H}\right), 5.90$ (ddt, $\left.J_{1}=16.7 J_{2}=10.5 J_{3}=7.1,1 \mathrm{H}\right)$, $7.15\left(\mathrm{dd}, J_{1}=8.0 J_{2}=1.8,1 \mathrm{H}\right), 7.33-7.40(\mathrm{~m}, 4 \mathrm{H}), 7.45(\mathrm{~d}, J=8.0,1 \mathrm{H}), 7.49-7.53(\mathrm{~m}, 2 \mathrm{H})$. ${ }^{13} \mathrm{C}$ NMR $\left(75.5 \mathrm{MHz}, \mathrm{CDCl}_{3}\right) \delta-5.0\left(\mathrm{CH}_{3}\right),-4.7\left(\mathrm{CH}_{3}\right), 18.3(\mathrm{C}), 20.9\left(\mathrm{CH}_{3}\right), 25.9$ $\left(3 \mathrm{xCH}_{3}\right), 44.5\left(\mathrm{CH}_{2}\right), 72.5(\mathrm{CH}), 87.5(\mathrm{C}), 93.6(\mathrm{C}), 116.7\left(\mathrm{CH}_{2}\right), 119.8(\mathrm{C}), 123.5(\mathrm{C})$, $126.0(\mathrm{CH}), 128.2(\mathrm{CH}), 128.4(2 \times \mathrm{CH}), 129.4(\mathrm{CH}), 131.4(2 \times \mathrm{CH}), 132.0(\mathrm{CH}), 135.6$ (CH), 136.2 (C), 144.2 (C). HRMS (ESI) calcd for $\mathrm{C}_{25} \mathrm{H}_{32} \mathrm{OSi}[\mathrm{M}+\mathrm{Na}]^{+}$: 399.2115, found: 399.2102 . 
g) General procedure for the intramolecular PKR:

To a solution of the corresponding protected alcohol 7d,l in dry DCM $(0.1 \mathrm{M}), \mathrm{Co}_{2}(\mathrm{CO}) 8$ ( 1 equiv.) was added and the reaction mixture was stirred at room temperature for 2 hours. Then, NMO (10 equiv.) was added and the reaction mixture was stirred overnight. Upon completion of the reaction, solvents were removed under reduced pressure and the residue purified by flash chromatography using mixtures of $n$ hexanes:ethyl acetate as eluent. The enantiomeric excess of the product was determined by HPLC analysis: Chiralpak IC (25 cm x $0.46 \mathrm{~cm}$ column), hexane:isopropanol 90:10 as eluent and flow $=1 \mathrm{ml} / \mathrm{min}$, unless otherwise indicated.

(3aS,5R)-5-((tert-Butyldimethylsilyl)oxy)-1-phenyl-3,3a,4,5-tetrahydro-2Hcyclopenta[a]naphthalen-2-one $(8 \mathrm{~d})$ :<smiles>O=C1C[C@H]2C[C@H](O[Sb])c3ccccc3C2=C1c1ccccc1</smiles>

Flash chromatography of the crude reaction product [n-hexane:EtOAc (100:1)] gave a white solid $(92 \%, 98 \%$ ee $) .[\alpha]_{\mathrm{D}}{ }^{25}=+77.8\left(\mathrm{c} 1.0 ; \mathrm{CHCl}_{3}\right)$. mp 140-142 ${ }^{\circ} \mathrm{C} .{ }^{1} \mathrm{H}$ NMR $(300$ $\left.\mathrm{MHz}_{\mathrm{CDCl}}\right) \delta 0.08(\mathrm{~s}, 3 \mathrm{H}), 0.14(\mathrm{~s}, 3 \mathrm{H}), 0.82(\mathrm{~s}, 9 \mathrm{H}), 1.88\left(\mathrm{td}, J_{1}=13.2 J_{2}=3.4,1 \mathrm{H}\right)$, $2.22\left(\mathrm{dd}, J_{1}=18.6 J_{2}=3.4,1 \mathrm{H}\right), 2.30\left(\mathrm{ddd}, J_{1}=13.2 J_{2}=4.0 J_{3}=2.5,1 \mathrm{H}\right), 2.83\left(\mathrm{dd}, J_{1}=18.6\right.$ $\left.J_{2}=6.8,1 \mathrm{H}\right), 3.53\left(\mathrm{ddt}, J_{1}=13.2 J_{2}=7.1 J_{3}=3.6,1 \mathrm{H}\right), 4.89(\mathrm{t}, J=2.9,1 \mathrm{H}), 6.91-6.97(\mathrm{~m}$, $1 \mathrm{H}), 7.12-7.18(\mathrm{~m}, 3 \mathrm{H}), 7.23-7.37(\mathrm{~m}, 5 \mathrm{H}) .{ }^{13} \mathrm{C} \mathrm{NMR}\left(75.5 \mathrm{MHz}, \mathrm{CDCl}_{3}\right) \delta-4.3\left(\mathrm{CH}_{3}\right)$,

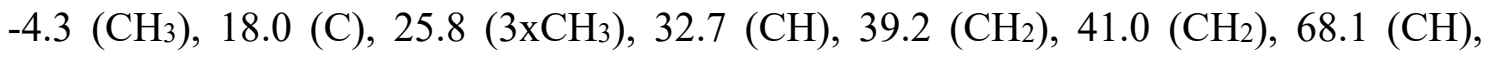
$127.4(\mathrm{CH}), 127.9(\mathrm{CH}), 128.4(\mathrm{CH}), 128.7(2 \mathrm{xCH}), 129.1(2 \mathrm{xCH}), 129.9(\mathrm{C}), 130.5$ (CH), $130.6(\mathrm{CH}), 132.9$ (C), $137.4(\mathrm{C}), 140.5$ (C), 166.8 (C), 206.6 (C). HRMS (ESI) calcd for $\mathrm{C}_{25} \mathrm{H}_{30} \mathrm{O}_{2} \mathrm{Si}[\mathrm{M}+\mathrm{H}]^{+}:$391.2088, found: 391.2082. The enantiomeric excess was determined as described in the intramolecular PKR procedure: $t_{\mathrm{R} \text { minor }}=22.4 \mathrm{~min}, t_{\mathrm{R}}$ major $=16.8 \mathrm{~min}$. 


\section{$2 H$-cyclopenta[a]naphthalen-2-one (81):}<smiles>Cc1ccc2c(c1)C1=C(c3ccccc3)C(=O)C[C@H]1C[C@H]2O[Sb]</smiles>

Flash chromatography of the crude reaction product [n-hexane:EtOAc (50:1)] gave a white solid $\left(81 \%, 98 \%\right.$ ee). mp $126-128{ }^{\circ} \mathrm{C}$. $[\alpha]_{\mathrm{D}}{ }^{25}=+18.9\left(\right.$ c $\left.1.0 ; \mathrm{CHCl}_{3}\right) .{ }^{1} \mathrm{H} \mathrm{NMR}$ $\left(300 \mathrm{MHz}, \mathrm{CDCl}_{3}\right) \delta 0.15(\mathrm{~s}, 3 \mathrm{H}), 0.21(\mathrm{~s}, 3 \mathrm{H}), 0.89(\mathrm{~s}, 9 \mathrm{H}), 1.93\left(\mathrm{td}, J_{1}=13.1 J_{2}=3.3\right.$, $1 \mathrm{H}), 2.04(\mathrm{~s}, 3 \mathrm{H}), 2.28\left(\mathrm{dd}, J_{1}=18.6 J_{2}=3.3,1 \mathrm{H}\right), 2.36\left(\mathrm{ddd}, J_{1}=13.1 J_{2}=4.0 J_{3}=2.4,1 \mathrm{H}\right)$, $2.90\left(\mathrm{dd}, J_{1}=18.6 J_{2}=6.8,1 \mathrm{H}\right), 3.59\left(\mathrm{ddt}, J_{1}=13.7 J_{2}=7.1 J_{3}=3.7,1 \mathrm{H}\right), 4.94(\mathrm{t}, J=2.9$, 1H), 6.98 (br s, 1H), 7.11-7.14 (m, 1H), 7.19-7.25 (m, 3H), 7.33-7.44 (m, 3H). ${ }^{13} \mathrm{C}$ NMR (75.5 MHz, $\left.\mathrm{CDCl}_{3}\right) \delta-4.3\left(\mathrm{CH}_{3}\right),-4.2\left(\mathrm{CH}_{3}\right), 18.0(\mathrm{C}), 21.0\left(\mathrm{CH}_{3}\right), 25.8\left(3 \mathrm{xCH}_{3}\right)$, $32.8(\mathrm{CH}), 39.4\left(\mathrm{CH}_{2}\right), 41.1\left(\mathrm{CH}_{2}\right), 68.0(\mathrm{CH}), 127.9(\mathrm{CH}), 128.7(2 \times \mathrm{CH}), 129.0(\mathrm{CH})$, $129.2(2 \times \mathrm{xH}), 129.7(\mathrm{C}), 130.4(\mathrm{CH}), 131.5(\mathrm{CH}), 133.0(\mathrm{C}), 136.9(\mathrm{C}), 137.3(\mathrm{C})$, 137.7 (C), 167.2 (C), 206.7 (C). HRMS (EI) calcd for $\mathrm{C}_{26} \mathrm{H}_{32} \mathrm{O}_{2} \mathrm{Si}[\mathrm{M}+\mathrm{H}]^{+}$: 405.2244 , found: 405.2236. The enantiomeric excess was determined as described in the intramolecular PKR procedure: $t_{\mathrm{R} \text { minor }}=25.6 \mathrm{~min}, t_{\mathrm{R} \text { major }}=17.8 \mathrm{~min}$.

\section{h) General procedure for the deprotection:}

To a solution of the corresponding protected alcohol 8d,l in THF (0.2M), TBAF $(1.2$ equiv.) was added at $0^{\circ} \mathrm{C}$ and the reaction mixture was stirred at room temperature. When the starting material was consumed (approximately 2-4 hours), the reaction mixture was quenched with water and extracted with diethyl ether. The organic layers were combined, dried over $\mathrm{Na}_{2} \mathrm{SO}_{4}$, filtered and evaporated under reduced pressure. The crude product was purified by flash chromatography employing mixtures of $n$ hexane:ethyl acetate as eluents. The enantiomeric excess of the product was determined by HPLC analysis: Chiralcel OD-H (25 cm x $0.46 \mathrm{~cm}$ column), hexane:isopropanol 90:10 as eluent and flow $=1 \mathrm{ml} / \mathrm{min}$. 

one (9d):<smiles>O=C1C[C@H]2C[C@H](O)c3ccccc3C2=C1c1ccccc1</smiles>

Flash chromatography of the crude reaction product [ $n$-hexane:EtOAc (3:1)] gave a white solid $\left(91 \%, 97 \%\right.$ ee). $[\alpha] \mathrm{D}^{25}=+43.9\left(\mathrm{c} 1.0 ; \mathrm{CHCl}_{3}\right)$. mp 170-172 ${ }^{\circ} \mathrm{C} .{ }^{1} \mathrm{H}$ NMR $(300$ $\mathrm{MHz}_{\mathrm{CDCl}}$ ) $\delta 1.63($ br s, $\mathrm{OH}), 1.91\left(\mathrm{td}, J_{1}=13.5 J_{2}=3.6,1 \mathrm{H}\right), 2.23\left(\mathrm{dd}, J_{1}=18.8 J_{2}=3.2\right.$, $1 \mathrm{H}), 2.45\left(\mathrm{ddd}, J_{1}=13.6 J_{2}=4.0 J_{3}=2.2,1 \mathrm{H}\right), 2.84\left(\mathrm{dd}, J_{1}=18.7 J_{2}=6.8,1 \mathrm{H}\right), 3.45$ (ddt, $\left.J_{1}=13.7 J_{2}=7.0 J_{3}=3.6,1 \mathrm{H}\right), 4.94\left(\mathrm{dd}, J_{1}=3.7 J_{2}=2.2,1 \mathrm{H}\right), 6.97-7.02(\mathrm{~m}, 1 \mathrm{H}), 7.14-7.17$ $(\mathrm{m}, 3 \mathrm{H}), 7.34-7.40(\mathrm{~m}, 5 \mathrm{H}) .{ }^{13} \mathrm{C} \mathrm{NMR}\left(75.5 \mathrm{MHz}, \mathrm{CDCl}_{3}\right) \delta 32.7(\mathrm{CH}), 38.2\left(\mathrm{CH}_{2}\right)$, $40.9\left(\mathrm{CH}_{2}\right), 67.6(\mathrm{CH}), 128.1(2 \mathrm{xCH}), 128.7(\mathrm{CH}), 128.8(2 \mathrm{xCH}), 129.1(2 \mathrm{xCH}), 130.1$ (C), $130.3(\mathrm{CH}), 131.1(\mathrm{CH}), 132.5$ (C), 137.8 (C), 139.7 (C), 166.0 (C), 206.5 (C). HRMS (ESI) calcd for $\mathrm{C}_{19} \mathrm{H}_{16} \mathrm{O}_{2}[\mathrm{M}+\mathrm{H}]^{+}:$277.1223, found: 277.1216. The enantiomeric excess was determined as described in the deprotection procedure: $t_{\mathrm{R}}$ minor $=24.2 \mathrm{~min}, t_{\mathrm{R} \text { major }}=18.6 \mathrm{~min}$.

(3aS,5R)-5-Hydroxy-8-methyl-1-phenyl-3,3a,4,5-tetrahydro-2Hcyclopenta $[a]$ naphthalen-2-one (91):<smiles>Cc1ccc2c(c1)C1=C(c3ccccc3)C(=O)C[C@H]1C[C@H]2O</smiles>

Flash chromatography of the crude reaction product [ $n$-hexane:EtOAc (5:1)] gave a white solid $\left(75 \%, 96 \%\right.$ ee). mp 97-99 ${ }^{\circ} \mathrm{C} .[\alpha]_{\mathrm{D}}{ }^{25}=-62.9\left(\mathrm{c} 1.0 ; \mathrm{CHCl}_{3}\right) .{ }^{1} \mathrm{H} \mathrm{NMR}(300$ $\left.\mathrm{MHz}_{\mathrm{CDCl}}\right) \delta 1.96\left(\mathrm{td}, J_{1}=13.5 J_{2}=3.6,1 \mathrm{H}\right), 2.05\left(\mathrm{~s}, \mathrm{CH}_{3}\right), 2.30\left(\mathrm{dd}, J_{1}=18.8 J_{2}=3.3\right.$, $1 \mathrm{H}), 2.51\left(\mathrm{ddd}, J_{1}=13.5 J_{2}=4.0 J_{3}=2.2,1 \mathrm{H}\right), 2.91\left(\mathrm{dd}, J_{1}=18.7 J_{2}=6.8,1 \mathrm{H}\right), 3.51$ (ddt, $\left.J_{1}=13.8 J_{2}=7.0 J_{3}=3.6,1 \mathrm{H}\right), 4.98(\mathrm{t}, J=2.9,1 \mathrm{H}), 7.00(\mathrm{~m}, 1 \mathrm{H}), 7.16-7.25(\mathrm{~m}, 3 \mathrm{H}), 7.33-$ $7.44(\mathrm{~m}, 4 \mathrm{H}) .{ }^{13} \mathrm{C}$ NMR (75.5 MHz, $\left.\mathrm{CDCl}_{3}\right) \delta 21.0\left(\mathrm{CH}_{3}\right), 32.7(\mathrm{CH}), 38.4\left(\mathrm{CH}_{2}\right), 40.9$ $\left(\mathrm{CH}_{2}\right), 67.4(\mathrm{CH}), 128.0(\mathrm{CH}), 128.7(2 \times \mathrm{CH}), 129.1(2 \times \mathrm{CH}), 129.2(\mathrm{CH}), 129.9(\mathrm{C})$, $130.1(\mathrm{CH}), 132.0(\mathrm{CH}), 132.6(\mathrm{C}), 136.9(\mathrm{C}), 137.7$ (C), $137.8(\mathrm{C}), 166.4(\mathrm{C}), 206.5$ 
(C). HRMS (ESI) calcd for $\mathrm{C}_{20} \mathrm{H}_{18} \mathrm{O}_{2}[\mathrm{M}+\mathrm{H}]^{+}$: 291.1380, found: 291.1378. The enantiomeric excess was determined as described in the deprotection procedure: $t_{\mathrm{R}}$ minor $=24.9 \mathrm{~min}, t_{\mathrm{R} \text { major }}=19.7 \mathrm{~min}$.

i) Procedure for the intramolecular hydroalkoxylation:

To a solution of IPrAuCl (5 mol\%) in dry DCM (0.1M), AgOTf (5 mol\%) was added. After stirring for $10 \mathrm{~min}$, a white precipitate was formed and the corresponding substrate 3d was added. Upon completion of the reaction (approximately 10 minutes), solvents were removed under reduced pressure and the residue purified by flash chromatography on deactivated silica gel (by adding $1 \% \mathrm{Et}_{3} \mathrm{~N}$ to the eluent) employing mixtures of $n$-hexane:ethyl acetate as eluents.

(R,Z)-1-Allyl-3-benzylidene-1,3-dihydroisobenzofuran (10d):<smiles>C=CCC1OC(=Cc2ccccc2)c2ccccc21</smiles>

Flash chromatography of the crude reaction product [ $n$-hexane] gave a colorless oil $(95 \%, 99 \%$ ee $) .[\alpha]_{\mathrm{D}}{ }^{25}=-30.5\left(\mathrm{c} 1.0 ; \mathrm{CHCl}_{3}\right) .{ }^{1} \mathrm{H} \mathrm{NMR}\left(300 \mathrm{MHz}, \mathrm{CDCl}_{3}\right) \delta 2.59-2.80$ (m, 2H), 5.14-5.25 (m, 2H), 5.69-5.73 (m, 1H), 5.85-5.99 (m, 2H), 7.12-7.17 (m, 1H), 7.28-7.39 (m, 5H), 7.55-7.58 (m, 1H), 7.73-7.77 (m, 2H). ${ }^{13} \mathrm{C}$ NMR (75.5 MHz, $\left.\mathrm{CDCl}_{3}\right)$ $\delta 40.3\left(\mathrm{CH}_{2}\right), 85.1(\mathrm{CH}), 96.0(\mathrm{CH}), 118.5\left(\mathrm{CH}_{2}\right), 119.9(\mathrm{CH}), 121.4(\mathrm{CH}), 125.2(\mathrm{CH})$, $127.8(2 \mathrm{xCH}), 128.3(\mathrm{CH}), 128.3(2 \mathrm{xCH}), 128.6(\mathrm{CH}), 132.8(\mathrm{CH}), 135.0(\mathrm{C}), 136.4$ (C), 142.0 (C), 155.3 (C). HRMS (ESI) calcd for $\mathrm{C}_{18} \mathrm{H}_{16} \mathrm{O}[\mathrm{M}+\mathrm{H}]^{+}: 249.1210$, found: 249.1216. The enantiomeric excess was determined with the aid of HPLC analysis: Chiralcel OD-H (25 cm x 0.46 cm column), hexane:isopropanol 99:1 as eluent and flow $=1 \mathrm{~mL} / \mathrm{min}: t_{\mathrm{R} \text { minor }}=10.3 \mathrm{~min}, t_{\mathrm{R} \text { major }}=8.6 \mathrm{~min}$.

(R,Z)-1-Benzylidene-3-(prop-2-yn-1-yl)-1,3-dihydroisobenzofuran (11d):<smiles>C#CCC1OC(=Cc2ccccc2)c2ccccc21</smiles> 
Flash chromatography of the crude reaction product [ $n$-hexane:EtOAc (50:1)] gave a colorless oil $\left(67 \%, 91 \%\right.$ ee). $[\alpha]_{\mathrm{D}}{ }^{25}=-95.0$ (c 1.0; $\left.\mathrm{CHCl}_{3}\right) .{ }^{1} \mathrm{H} \mathrm{NMR}\left(300 \mathrm{MHz}, \mathrm{CDCl}_{3}\right)$ $\delta 2.09(\mathrm{t}, J=2.7,1 \mathrm{H}), 2.72\left(\mathrm{ddd}, J_{1}=16.6 J_{2}=7.4 J_{3}=2.7,1 \mathrm{H}\right), 2.93\left(\mathrm{ddd}, J_{1}=16.6 J_{2}=5.4\right.$ $\left.J_{3}=2.7,1 \mathrm{H}\right), 5.75\left(\mathrm{dd}, J_{1}=7.4 J_{2}=5.4,1 \mathrm{H}\right), 5.94(\mathrm{~s}, 1 \mathrm{H}), 7.13-7.18(\mathrm{~m}, 1 \mathrm{H}), 7.31-7.40$ $(\mathrm{m}, 5 \mathrm{H}), 7.52-7.59(\mathrm{~m}, 2 \mathrm{H}), 7.73-7.76(\mathrm{~m}, 1 \mathrm{H}) .{ }^{13} \mathrm{C} \mathrm{NMR}\left(75.5 \mathrm{MHz}, \mathrm{CD}_{2} \mathrm{Cl}_{2}\right) \delta 26.6$ $\left(\mathrm{CH}_{2}\right), 71.3(\mathrm{CH}), 79.6(\mathrm{C}), 83.7(\mathrm{CH}), 96.8(\mathrm{CH}), 120.4(\mathrm{CH}), 122.4(\mathrm{CH}), 126.0(\mathrm{CH})$, $128.4(2 \times C H), 128.9$ (2xCH), $129.4(\mathrm{CH}), 129.4(\mathrm{CH}), 130.1(\mathrm{C}), 136.8(\mathrm{C}), 141.7(\mathrm{C})$, 155.6 (C). HRMS (ESI) calcd for $\mathrm{C}_{18} \mathrm{H}_{14} \mathrm{O}[\mathrm{M}+\mathrm{H}]^{+}: 247.1117$, found: 247.1110. The enantiomeric excess was determined with the aid of HPLC analysis: Chiralpak IC (25 $\mathrm{cm} \times 0.46 \mathrm{~cm}$ column), hexane:isopropanol $99: 1$ as eluent and flow $=1 \mathrm{~mL} / \mathrm{min}: t_{\mathrm{R}}$ minor $=6.5 \mathrm{~min}, t_{\mathrm{R} \text { major }}=5.9 \mathrm{~min}$.

\section{Determination of the absolute configuration of compounds $3 d$ and $5 d$ by X-ray diffraction analysis}

Suitable crystals for X-ray analysis were obtained by slow evaporation of solutions of 3d and $\mathbf{5 d}$ in hexane.

Intensity data were collected on an Agilent Technologies Super-Nova diffractometer, which was equipped with monochromated $\mathrm{Cu} k \alpha$ radiation $(\lambda=1.54184 \AA)$ and Atlas CCD detector. Measurements were carried out at 100(2) K with the help of an Oxford Cryostream 700 PLUS temperature device. Data frames were processed (unit cell determination, analytical absorption correction with face indexing, intensity data integration and correction for Lorentz and polarization effects) using the CrysAlis software package. ${ }^{5}$ The structure was solved using Superflip ${ }^{6}$ and refined by full-matrix least-squares with SHELXL-97. ${ }^{7}$ Final geometrical calculations were carried out with Mercury ${ }^{8}$ and PLATON $^{9}$ as integrated in WinGX. ${ }^{10}$ 


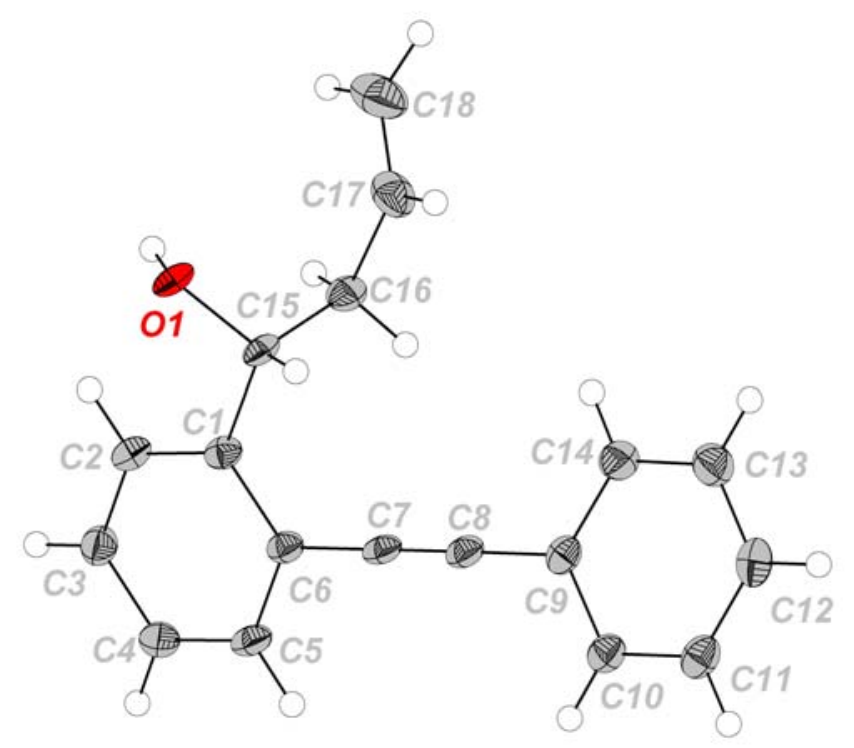

Figure S1. ORTEP diagram of $\mathbf{3 d}$.

Table S3. Crystal and refinement data for 3d.

Crystal Data Formula

Formula Weight

Crystal System

Space group

a, b, c $(\AA)$

$\alpha, \beta, y\left(^{\circ}\right)$

$\mathrm{V}\left(\AA^{3}\right)$

Z

$\mathrm{D}$ (calc) $\left(\mathrm{g} \cdot \mathrm{cm}^{-3}\right)$

$\mu(\mathrm{CuK \alpha})\left(\mathrm{mm}^{-1}\right)$

$\mathrm{F}(000)$

Crystal Size $(\mathrm{mm})$

Data Collection Temperature $(\mathrm{K})$

Radiation $(\AA)$ CuKa

Interval of $\theta\left(^{\circ}\right)$

Dataset

Tot., Uniq. Data, R(int)

Observed data $[I>2 \sigma(I)]$

Refinement Nref, Npar

$R(F), R_{w}\left(F^{2}\right), S(G O F)$

$w=1 /\left[\sigma^{2}\left(F_{0}^{2}\right)+(0.0836 P)^{2}+0.0926 P\right]$ where

Max. and Av. Shift/Error

Flack $x$

Hooft y

Min. and Max. Resd. Dens. $\left(e \AA^{-3}\right)$
$\mathrm{C}_{18} \mathrm{H}_{16} \mathrm{O}$

248.31

Monoclinic

P21 (No. 4)

$10.5879(2) 4.8182(1) 13.6534(2)$

$9096.876(2) 90$

691.51(2)

2

1.192

0.559

264

$0.31 \times 0.06 \times 0.05$

$100(1)^{\circ}$

1.54184

$3.3,72.5$

$-13: 12 ;-5: 5 ;-16: 12$

$13261,2711(0.032)$

2582

2711,181

$0.0464,0.1219,1.06$

$\mathrm{P}=\left[\operatorname{Max}\left(\mathrm{F}_{\mathrm{o}}{ }^{2}, 0+2 \mathrm{~F}_{\mathrm{c}}{ }^{2}\right) / 3\right.$

$0.00,0.00$

$0.2(3)$

$0.08(14)$

$-0.19,0.33$ 


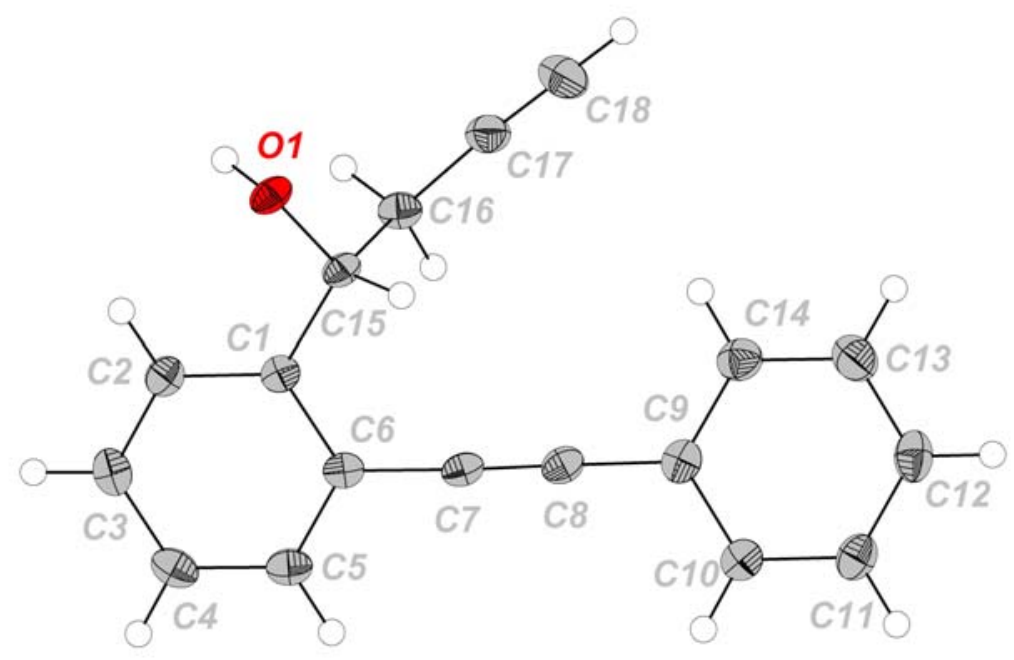

Figure S2. ORTEP diagram of 5d.

Table S4. Crystal and refinement data for $\mathbf{5 d}$.

Crystal Data Formula

Formula Weight

Crystal System

Space group

$a, b, c(\AA)$

$\alpha, \beta, \gamma\left({ }^{\circ}\right)$

$\mathrm{V}\left(\AA^{3}\right)$

$Z$

$\mathrm{D}$ (calc) $\left(\mathrm{g} \cdot \mathrm{cm}^{-3}\right)$

$\mu(\mathrm{CuK \alpha})\left(\mathrm{mm}^{-1}\right)$

$\mathrm{F}(000)$

Crystal Size $(\mathrm{mm})$

Data Collection Temperature $(\mathrm{K})$

$\mathrm{D}$ (calc) $\left(\mathrm{g} \cdot \mathrm{cm}^{-3}\right)$

$\mu($ CuKa $)\left(\mathrm{mm}^{-1}\right)$

Dataset

Tot., Uniq. Data, R(int)

Observed data $[I>2 \sigma(I)]$
$\mathrm{C}_{18} \mathrm{H}_{14} \mathrm{O}$

246.29

Monoclinic

P21 (No. 4)

10.5044(2) 4.7708(1) 13.7318(3)

$9097.605(2) 90$

$682.11(2)$

2

1.199

0.566

260

$0.37 \times 0.06 \times 0.05$

100

1.54184

$3.3,72.4$

$-12: 12 ;-5: 5 ;-16: 16$

$13241,2623,0.032$

2532

2623, 176

$0.0309,0.0807,1.07$

Refinement Nref, Npar

$R(F), R_{w}\left(F^{2}\right), S(G O F)$

$\mathrm{w}=1 /\left[\sigma^{2}\left(\mathrm{~F}_{\mathrm{o}}^{2}\right)+(0.0462 \mathrm{P})^{2}+0.0988 \mathrm{P}\right]$ where $\mathrm{P}=\left[\operatorname{Max}\left(\mathrm{F}_{\mathrm{o}}^{2}, 0+2 \mathrm{~F}_{\mathrm{c}}^{2}\right) / 3\right.$

Max. and Av. Shift/Error

$0.00,0.00$

Flack $x$

$0.1(2)$

Hooft y

$0.12(10)$

Min. and Max. Resd. Dens. $\left(\mathrm{e}^{-3}\right)$

$-0.15,0.16$

For both structures, analysis of the absolute structure using likelihood methods ${ }^{11}$ were performed using Olex2. ${ }^{12}$ The results indicated that the absolute structures had been 
correctly assigned. The method calculated that the probability that the structures are inverted is smaller than $10^{-9}$ and $10^{-16}$, respectively. The absolute structure parameter $y^{10}$ was calculated using Olex2. ${ }^{12}$ The resulting values were $y=0.08$ (14) and 0.12(10), respectively, which together with Flack parameter values, indicate that the absolute structures have probably been determined correctly.

\section{Full list of authors in the Gaussian 09 reference}

Gaussian 09, Revision D.01,

M. J. Frisch, G. W. Trucks, H. B. Schlegel, G. E. Scuseria, M. A. Robb, J. R. Cheeseman, G. Scalmani, V. Barone, B. Mennucci, G. A. Petersson, H. Nakatsuji, M. Caricato, X. Li, H. P. Hratchian, A. F. Izmaylov, J. Bloino, G. Zheng, J. L. Sonnenberg, M. Hada, M. Ehara, K. Toyota, R. Fukuda, J. Hasegawa, M. Ishida, T. Nakajima, Y. Honda, O. Kitao, H. Nakai, T. Vreven, J. A. Montgomery, Jr., J. E. Peralta, F. Ogliaro, M. Bearpark, J. J. Heyd, E. Brothers, K. N. Kudin, V. N. Staroverov, T. Keith, R. Kobayashi, J. Normand, K. Raghavachari, A. Rendell, J. C. Burant, S. S. Iyengar, J. Tomasi, M. Cossi, N. Rega, J. M. Millam, M. Klene, J. E. Knox, J. B. Cross, V. Bakken, C. Adamo, J. Jaramillo, R. Gomperts, R. E. Stratmann, O. Yazyev, A. J. Austin, R. Cammi, C. Pomelli, J. W. Ochterski, R. L. Martin, K. Morokuma, V. G. Zakrzewski, G. A. Voth, P. Salvador, J. J. Dannenberg, S. Dapprich, A. D. Daniels, O. Farkas, J. B. Foresman, J. V. Ortiz, J. Cioslowski, and D. J. Fox, Gaussian, Inc., Wallingford CT, 2013.

\section{Computational Data}

Table S5 Preferred conformations of o-alkynyl benzaldehydes using M06-2X (solution phase) and B3LYP (gas phase) methods.. All free energies in $\mathrm{kcal} \mathrm{mol}^{-1}$.<smiles>[R]C#Cc1ccccc1C=O</smiles>

Conformation A<smiles>[R]C#Cc1ccccc1C=O</smiles>

Conformation B

M06-2X/6-311+G(d,p)-IEFPCM//B3LYP/6-31G(d)

\begin{tabular}{cccc}
\hline & \multicolumn{3}{c}{ Conformation } \\
R & A & BCM & Toluene \\
\hline H (1a) & 0 & 1.6 & 2.2 \\
TMS (1e) & 0 & 1.6 & 2.3 \\
Cl (1g) & 0 & 1.2 & 1.8
\end{tabular}


B3LYP/6-31G(d)

\begin{tabular}{ccc}
\hline & \multicolumn{2}{c}{ Conformation } \\
$\mathrm{R}$ & $\mathrm{A}$ & $\mathrm{B}$ \\
\hline $\mathrm{H}(\mathbf{1 a})$ & 0 & 2.4 \\
$\mathrm{TMS}(\mathbf{1 e})$ & 0 & 2.3 \\
$\mathrm{Cl}(\mathbf{1 g})$ & 0 & 2.0
\end{tabular}

\section{$1 g$ conformation A}

B3LYP Energy $=$

$-881.303336$

B3LYP Free Energy =

$-881.228244$

M06-2X Derived Solution Free Energy $(\mathrm{DCM})=$

$-881.172013$

M06-2X Derived Solution Free Energy (toluene) $=$

$-881.169366$

Number of Imaginary Frequencies $=$

B3LYP/6-31G(d) Geometry

$\begin{array}{lrrr}\mathrm{C} & -0.838047 & -1.828803 & 0.000005 \\ \mathrm{C} & -0.317303 & -0.520635 & 0.000020 \\ \mathrm{C} & -1.217259 & 0.573653 & 0.000003 \\ \mathrm{C} & -2.597992 & 0.337658 & -0.000006 \\ \mathrm{C} & -3.098998 & -0.958994 & -0.000011 \\ \mathrm{C} & -2.213065 & -2.042539 & -0.000004 \\ \mathrm{H} & -0.150292 & -2.668370 & 0.000009 \\ \mathrm{H} & -3.257288 & 1.200207 & -0.000011 \\ \mathrm{H} & -4.171527 & -1.130651 & -0.000022 \\ \mathrm{H} & -2.596167 & -3.059272 & -0.000008 \\ \mathrm{C} & -0.730859 & 1.975600 & 0.000009 \\ \mathrm{H} & 0.370061 & 2.096203 & 0.000017 \\ \mathrm{O} & -1.465854 & 2.946035 & -0.000006 \\ \mathrm{C} & 1.100659 & -0.338847 & 0.000041 \\ \mathrm{C} & 2.308447 & -0.246989 & -0.000042 \\ \mathrm{Cl} & 3.950503 & -0.100413 & -0.000002\end{array}$

1 1g conformation B

B3LYP Energy =

$-881.300140$

B3LYP Free Energy =

$-881.225104$

M06-2X Derived Solution Free Energy $($ DCM $)=$

$-881.170093$

M06-2X Derived Solution Free Energy (toluene) =

$-881.166531$

Number of Imaginary Frequencies $=$

B3LYP/6-31G(d) Geometry

$\begin{array}{lrrr}\mathrm{C} & -1.112418 & -1.659274 & 0.000168 \\ \mathrm{C} & -0.492288 & -0.395663 & 0.000040 \\ \mathrm{C} & -1.315726 & 0.761279 & -0.000007 \\ \mathrm{C} & -2.709038 & 0.616349 & 0.000076 \\ \mathrm{C} & -3.307970 & -0.639845 & 0.000204 \\ \mathrm{C} & -2.499982 & -1.779530 & 0.000251 \\ \mathrm{H} & -0.485869 & -2.545474 & 0.000204\end{array}$




$\begin{array}{lrrr}\mathrm{H} & -3.325115 & 1.513113 & 0.000038 \\ \mathrm{H} & -4.389989 & -0.732158 & 0.000265 \\ \mathrm{H} & -2.951091 & -2.767959 & 0.000351 \\ \mathrm{C} & -0.776115 & 2.140877 & -0.000136 \\ \mathrm{H} & -1.568473 & 2.923660 & -0.000149 \\ \mathrm{O} & 0.397426 & 2.452214 & -0.000202 \\ \mathrm{C} & 0.932455 & -0.339133 & -0.000025 \\ \mathrm{C} & 2.140003 & -0.429049 & -0.000161 \\ \mathrm{Cl} & 3.787506 & -0.450880 & -0.000091\end{array}$

\section{1a conformation $A$}

B3LYP Energy =

$-421.716074$

B3LYP Free Energy =

M06-2X Derived Solution Free Energy $(\mathrm{DCM})=$

M06-2X Derived Solution Free Energy (toluene) $=$ $-421.570604$

Number of Imaginary Frequencies =

B3LYP/6-31G(d) Geometry

$\begin{array}{lrrr}\mathrm{C} & -0.656024 & -1.736251 & 0.000043 \\ \mathrm{C} & 0.347639 & -0.749687 & 0.000019 \\ \mathrm{C} & -0.033680 & 0.614399 & -0.000026 \\ \mathrm{C} & -1.392122 & 0.955892 & -0.000057 \\ \mathrm{C} & -2.373791 & -0.028516 & -0.000036 \\ \mathrm{C} & -2.000772 & -1.377336 & 0.000020 \\ \mathrm{H} & -0.364861 & -2.781790 & 0.000078 \\ \mathrm{H} & -1.647564 & 2.011092 & -0.000096 \\ \mathrm{H} & -3.424446 & 0.247356 & -0.000058 \\ \mathrm{H} & -2.761787 & -2.152874 & 0.000038 \\ \mathrm{C} & 0.977411 & 1.701360 & -0.000056 \\ \mathrm{H} & 2.033073 & 1.368099 & 0.000055 \\ \mathrm{O} & 0.695040 & 2.885849 & 0.000075 \\ \mathrm{C} & 1.719558 & -1.155028 & 0.000050 \\ \mathrm{C} & 2.865985 & -1.542423 & 0.000026 \\ \mathrm{H} & 3.880050 & -1.873136 & -0.000518\end{array}$

\section{1a conformation B}

B3LYP Energy $=$

$-421.712046$

B3LYP Free Energy =

$-421.626526$

$-421.571168$

M06-2X Derived Solution Free Energy $(\mathrm{DCM})=$

$-421.567021$

Number of Imaginary Frequencies $=$

B3LYP/6-31G(d) Geometry

$\begin{array}{lrrr}\mathrm{C} & -0.959178 & -1.496087 & 0.000018 \\ \mathrm{C} & 0.149453 & -0.629416 & 0.000016 \\ \mathrm{C} & -0.088025 & 0.770242 & 0.000006 \\ \mathrm{C} & -1.405700 & 1.246266 & -0.000019 \\ \mathrm{C} & -2.492174 & 0.376556 & -0.000028 \\ \mathrm{C} & -2.261181 & -1.000992 & -0.000006 \\ \mathrm{H} & -0.780205 & -2.566498 & 0.000037 \\ \mathrm{H} & -1.569690 & 2.321803 & -0.000029\end{array}$




$\begin{array}{lrrr}\mathrm{H} & -3.506365 & 0.764860 & -0.000046 \\ \mathrm{H} & -3.097625 & -1.694463 & -0.000007 \\ \mathrm{C} & 0.995363 & 1.781664 & 0.000023 \\ \mathrm{H} & 0.616721 & 2.829454 & -0.000019 \\ \mathrm{O} & 2.188365 & 1.558280 & -0.000005 \\ \mathrm{C} & 1.454937 & -1.207277 & 0.000060 \\ \mathrm{C} & 2.502033 & -1.813256 & 0.000039 \\ \mathrm{H} & 3.457072 & -2.287591 & -0.000556\end{array}$

\section{1e conformation A}

B3LYP Energy =

$-830.416350$

B3LYP Free Energy =

$-830.235740$

M06-2X Derived Solution Free Energy $(\mathrm{DCM})=$

M06-2X Derived Solution Free Energy (toluene) = $-830.125972$

Number of Imaginary Frequencies =

B3LYP/6-31G(d) Geometry

$\begin{array}{lrrr}\mathrm{C} & 1.947559 & -1.842397 & 0.001257 \\ \mathrm{C} & 1.430084 & -0.532166 & 0.001899 \\ \mathrm{C} & 2.336219 & 0.558040 & 0.000809 \\ \mathrm{C} & 3.716041 & 0.316787 & -0.001042 \\ \mathrm{C} & 4.212061 & -0.981756 & -0.001791 \\ \mathrm{C} & 3.321502 & -2.061769 & -0.000576 \\ \mathrm{H} & 1.256007 & -2.678889 & 0.002031 \\ \mathrm{H} & 4.378747 & 1.176795 & -0.001927 \\ \mathrm{H} & 5.283953 & -1.157769 & -0.003329 \\ \mathrm{H} & 3.700837 & -3.080022 & -0.001115 \\ \mathrm{C} & 1.854967 & 1.961476 & 0.001759 \\ \mathrm{H} & 0.754580 & 2.084419 & 0.003797 \\ \mathrm{O} & 2.593481 & 2.929917 & 0.000572 \\ \mathrm{C} & 0.013634 & -0.345572 & 0.002952 \\ \mathrm{C} & -1.202719 & -0.232529 & 0.003026 \\ \mathrm{Si} & -3.039997 & -0.058859 & -0.000005 \\ \mathrm{C} & -3.772566 & -1.487146 & -0.996968 \\ \mathrm{H} & -3.508861 & -2.456627 & -0.559354 \\ \mathrm{H} & -4.867222 & -1.419103 & -1.023799 \\ \mathrm{H} & -3.411607 & -1.477666 & -2.031583 \\ \mathrm{C} & -3.484039 & 1.596465 & -0.794696 \\ \mathrm{H} & -3.050715 & 2.435782 & -0.239094 \\ \mathrm{H} & -3.118352 & 1.654193 & -1.826088 \\ \mathrm{H} & -4.571905 & 1.736800 & -0.817190 \\ \mathrm{C} & -3.652699 & -0.121263 & 1.786370 \\ \mathrm{H} & -4.744515 & -0.020414 & 1.823418 \\ \mathrm{H} & -3.387772 & -1.070189 & 2.266101 \\ \mathrm{H} & -3.221333 & 0.688364 & 2.385633\end{array}$

\section{1e conformation B}

B3LYP Energy = 
B3LYP/6-31G(d) Geometry

\begin{tabular}{|c|c|c|c|}
\hline $\mathrm{C}$ & 2.142947 & -1.687799 & -0.001291 \\
\hline $\mathrm{C}$ & 1.558629 & -0.406340 & -0.001551 \\
\hline $\mathrm{C}$ & 2.417389 & 0.725986 & -0.000491 \\
\hline $\mathrm{C}$ & 3.806001 & 0.538607 & 0.000712 \\
\hline $\mathrm{C}$ & 4.367617 & -0.734576 & 0.001026 \\
\hline $\mathrm{C}$ & 3.525964 & -1.849819 & 0.000020 \\
\hline $\mathrm{H}$ & 1.489572 & -2.554429 & -0.002042 \\
\hline $\mathrm{H}$ & 4.449014 & 1.416369 & 0.001501 \\
\hline $\mathrm{H}$ & 5.446498 & -0.858709 & 0.002066 \\
\hline $\mathrm{H}$ & 3.947598 & -2.851265 & 0.000291 \\
\hline $\mathrm{C}$ & 1.926471 & 2.123714 & -0.000344 \\
\hline $\mathrm{H}$ & 2.748744 & 2.875595 & 0.000012 \\
\hline $\mathrm{O}$ & 0.766628 & 2.483291 & -0.000475 \\
\hline $\mathrm{C}$ & 0.137088 & -0.311008 & -0.002531 \\
\hline $\mathrm{C}$ & -1.083661 & -0.331333 & -0.003050 \\
\hline $\mathrm{Si}$ & -2.919862 & -0.188634 & 0.000075 \\
\hline $\mathrm{C}$ & -3.673070 & -1.920807 & -0.090194 \\
\hline $\mathrm{H}$ & -3.363160 & -2.537053 & 0.761560 \\
\hline $\mathrm{H}$ & -4.768912 & -1.868188 & -0.082819 \\
\hline $\mathrm{H}$ & -3.370454 & -2.440207 & -1.006654 \\
\hline $\mathrm{C}$ & -3.437907 & 0.831257 & -1.503261 \\
\hline $\mathrm{H}$ & -2.978474 & 1.825470 & -1.479065 \\
\hline $\mathrm{H}$ & -3.135266 & 0.345995 & -2.438101 \\
\hline $\mathrm{H}$ & -4.527036 & 0.961424 & -1.529877 \\
\hline $\mathrm{C}$ & -3.443012 & 0.672428 & 1.598374 \\
\hline $\mathrm{H}$ & -4.532331 & 0.798671 & 1.635070 \\
\hline $\mathrm{H}$ & -3.142662 & 0.094541 & 2.479719 \\
\hline $\mathrm{H}$ & -2.984835 & 1.664470 & 1.676565 \\
\hline
\end{tabular}

\section{1d allylboration TS 1}

B3LYP Energy =

$-3456.170733$

B3LYP Free Energy =

$-3454.943569$

$-3454.464009$

M06-2X Derived Solution Free Energy $(\mathrm{DCM})=$ M06-2X Derived Solution Free Energy (toluene) $=$ $-3454.454125$ Number of Imaginary Frequencies =

$1(-312.87)$

B3LYP/6-31G(d) Geometry

$\begin{array}{lrrr}\text { B } & -0.310090 & 3.456398 & -0.501687 \\ \mathrm{C} & 0.149193 & 3.524320 & 1.167406 \\ \mathrm{C} & -0.406073 & 4.786051 & 1.584376 \\ \mathrm{C} & -1.727306 & 4.960176 & 1.933810 \\ \mathrm{H} & 1.240441 & 3.491584 & 1.158345 \\ \mathrm{H} & -0.267587 & 2.645224 & 1.662445 \\ \mathrm{H} & 0.184684 & 5.676597 & 1.368581 \\ \mathrm{H} & -2.112151 & 5.953233 & 2.150707 \\ \mathrm{H} & -2.298263 & 4.126371 & 2.331570 \\ \mathrm{O} & 0.175848 & 4.609246 & -1.203658 \\ \mathrm{C} & 0.607268 & 4.196568 & -2.512357 \\ \mathrm{O} & 0.154038 & 2.286360 & -1.224322 \\ \mathrm{C} & 1.088988 & 2.722937 & -2.254183\end{array}$




\begin{tabular}{|c|c|c|c|}
\hline $\mathrm{C}$ & -0.591456 & 4.272351 & -3.474468 \\
\hline $\mathrm{H}$ & -1.384434 & 3.578565 & -3.180078 \\
\hline $\mathrm{H}$ & -0.998712 & 5.288871 & -3.448513 \\
\hline $\mathrm{H}$ & -0.302307 & 4.050844 & -4.507532 \\
\hline $\mathrm{C}$ & 1.703689 & 5.154891 & -2.980455 \\
\hline $\mathrm{H}$ & 1.281438 & 6.156756 & -3.113947 \\
\hline $\mathrm{H}$ & 2.512535 & 5.226759 & -2.249691 \\
\hline $\mathrm{H}$ & 2.125273 & 4.836388 & -3.941023 \\
\hline $\mathrm{C}$ & 0.972289 & 1.790769 & -3.460243 \\
\hline $\mathrm{H}$ & -0.059376 & 1.709848 & -3.809265 \\
\hline $\mathrm{H}$ & 1.318842 & 0.786381 & -3.198525 \\
\hline $\mathrm{H}$ & 1.593630 & 2.158373 & -4.285234 \\
\hline $\mathrm{C}$ & 2.504361 & 2.648035 & -1.667011 \\
\hline $\mathrm{H}$ & 2.625080 & 3.340757 & -0.829515 \\
\hline $\mathrm{H}$ & 2.696210 & 1.635916 & -1.298463 \\
\hline $\mathrm{H}$ & 3.264434 & 2.882494 & -2.419624 \\
\hline $\mathrm{O}$ & -1.825807 & 3.408265 & -0.381678 \\
\hline $\mathrm{C}$ & -2.423006 & 4.523608 & -0.107874 \\
\hline $\mathrm{H}$ & -1.977875 & 5.424389 & -0.532313 \\
\hline $\mathrm{C}$ & -3.886895 & 4.569183 & 0.071209 \\
\hline $\mathrm{C}$ & -4.536904 & 5.670838 & -0.507166 \\
\hline $\mathrm{C}$ & -4.660736 & 3.581834 & 0.739924 \\
\hline $\mathrm{C}$ & -5.922239 & 5.797871 & -0.480045 \\
\hline $\mathrm{C}$ & -6.063520 & 3.727560 & 0.754353 \\
\hline $\mathrm{C}$ & -6.687411 & 4.812701 & 0.148319 \\
\hline $\mathrm{H}$ & -3.937551 & 6.428808 & -1.005542 \\
\hline $\mathrm{H}$ & -6.399750 & 6.653627 & -0.947927 \\
\hline $\mathrm{H}$ & -6.652059 & 2.972015 & 1.264549 \\
\hline $\mathrm{H}$ & -7.770384 & 4.894419 & 0.176165 \\
\hline $\mathrm{O}$ & 0.037781 & -2.516594 & 0.194083 \\
\hline $\mathrm{P}$ & 0.750551 & -1.062454 & 0.119656 \\
\hline $\mathrm{O}$ & 2.203819 & -1.389050 & -0.553777 \\
\hline $\mathrm{O}$ & 0.787362 & -0.418044 & 1.450530 \\
\hline $\mathrm{O}$ & 0.020581 & -0.307369 & -1.054801 \\
\hline $\mathrm{H}$ & 0.046952 & 0.706829 & -1.013513 \\
\hline $\mathrm{C}$ & 0.140234 & -3.477266 & -0.810089 \\
\hline $\mathrm{C}$ & 1.382988 & -4.093934 & -1.038967 \\
\hline $\mathrm{C}$ & -1.037256 & -3.890623 & -1.454641 \\
\hline $\mathrm{C}$ & 1.439700 & -5.114776 & -2.001943 \\
\hline $\mathrm{C}$ & -0.928880 & -4.925359 & -2.394849 \\
\hline $\mathrm{C}$ & 0.297025 & -5.523954 & -2.680183 \\
\hline $\mathrm{H}$ & 2.397724 & -5.578552 & -2.216845 \\
\hline $\mathrm{H}$ & -1.831244 & -5.268232 & -2.892162 \\
\hline $\mathrm{H}$ & 0.358207 & -6.315256 & -3.421956 \\
\hline $\mathrm{C}$ & 2.999526 & -2.413874 & -0.042566 \\
\hline $\mathrm{C}$ & 2.606434 & -3.744643 & -0.271975 \\
\hline $\mathrm{C}$ & 4.220405 & -2.084016 & 0.567818 \\
\hline $\mathrm{C}$ & 3.446651 & -4.766978 & 0.197962 \\
\hline $\mathrm{C}$ & 5.029868 & -3.141623 & 1.007159 \\
\hline $\mathrm{C}$ & 4.643646 & -4.470505 & 0.840644 \\
\hline $\mathrm{H}$ & 3.141983 & -5.800017 & 0.060015 \\
\hline $\mathrm{H}$ & 5.982106 & -2.905754 & 1.473342 \\
\hline $\mathrm{H}$ & 5.280816 & -5.272812 & 1.201779 \\
\hline
\end{tabular}




\begin{tabular}{|c|c|c|c|}
\hline $\mathrm{C}$ & 4.724023 & -0.672753 & 0.680962 \\
\hline $\mathrm{C}$ & 4.573812 & 0.054427 & 1.883209 \\
\hline $\mathrm{C}$ & 5.437229 & -0.109470 & -0.404736 \\
\hline $\mathrm{C}$ & 5.143960 & 1.330760 & 1.968594 \\
\hline $\mathrm{C}$ & 5.982868 & 1.171035 & -0.264880 \\
\hline $\mathrm{C}$ & 5.851823 & 1.909558 & 0.914300 \\
\hline $\mathrm{H}$ & 5.039790 & 1.893684 & 2.892844 \\
\hline $\mathrm{H}$ & 6.539023 & 1.595506 & -1.097466 \\
\hline $\mathrm{C}$ & -2.392920 & -3.336739 & -1.116489 \\
\hline $\mathrm{C}$ & -3.021178 & -2.396088 & -1.967895 \\
\hline $\mathrm{C}$ & -3.076468 & -3.827928 & 0.018406 \\
\hline $\mathrm{C}$ & -4.320986 & -1.978172 & -1.666127 \\
\hline $\mathrm{C}$ & -4.370538 & -3.363172 & 0.280691 \\
\hline $\mathrm{C}$ & -5.015163 & -2.443747 & -0.546048 \\
\hline $\mathrm{H}$ & -4.805659 & -1.263024 & -2.324830 \\
\hline $\mathrm{H}$ & -4.896355 & -3.735855 & 1.157183 \\
\hline $\mathrm{C}$ & 3.864353 & -0.531470 & 3.104293 \\
\hline $\mathrm{C}$ & 2.901625 & 0.460583 & 3.780183 \\
\hline $\mathrm{C}$ & 4.890760 & -1.068206 & 4.123544 \\
\hline $\mathrm{H}$ & 3.257576 & -1.376278 & 2.764687 \\
\hline $\mathrm{H}$ & 2.164938 & 0.833956 & 3.065628 \\
\hline $\mathrm{H}$ & 2.359801 & -0.044147 & 4.589249 \\
\hline $\mathrm{H}$ & 3.431354 & 1.310148 & 4.227809 \\
\hline $\mathrm{H}$ & 5.538095 & -1.833473 & 3.681639 \\
\hline $\mathrm{H}$ & 5.533763 & -0.260162 & 4.493800 \\
\hline $\mathrm{H}$ & 4.380063 & -1.513997 & 4.985880 \\
\hline $\mathrm{C}$ & 5.677232 & -0.873745 & -1.707867 \\
\hline $\mathrm{C}$ & 7.169170 & -1.221443 & -1.880957 \\
\hline $\mathrm{C}$ & 5.138856 & -0.124786 & -2.940595 \\
\hline $\mathrm{H}$ & 5.134044 & -1.821196 & -1.652052 \\
\hline $\mathrm{H}$ & 7.541424 & -1.802715 & -1.029929 \\
\hline $\mathrm{H}$ & 7.323788 & -1.813914 & -2.791033 \\
\hline $\mathrm{H}$ & 7.784977 & -0.317628 & -1.961557 \\
\hline $\mathrm{H}$ & 4.066849 & 0.073292 & -2.842242 \\
\hline $\mathrm{H}$ & 5.647658 & 0.835002 & -3.090201 \\
\hline $\mathrm{H}$ & 5.292403 & -0.724224 & -3.846218 \\
\hline $\mathrm{C}$ & 6.482325 & 3.288064 & 1.068816 \\
\hline $\mathrm{C}$ & 5.956206 & 4.294466 & 0.029037 \\
\hline $\mathrm{C}$ & 8.021105 & 3.213397 & 1.032284 \\
\hline $\mathrm{H}$ & 6.195934 & 3.662158 & 2.061310 \\
\hline $\mathrm{H}$ & 4.864964 & 4.378667 & 0.076084 \\
\hline $\mathrm{H}$ & 6.383589 & 5.289440 & 0.202971 \\
\hline $\mathrm{H}$ & 6.223718 & 3.992412 & -0.990548 \\
\hline $\mathrm{H}$ & 8.401491 & 2.528342 & 1.797841 \\
\hline $\mathrm{H}$ & 8.377595 & 2.856101 & 0.058688 \\
\hline $\mathrm{H}$ & 8.462986 & 4.201913 & 1.207523 \\
\hline $\mathrm{C}$ & -2.467937 & -4.869508 & 0.956759 \\
\hline $\mathrm{C}$ & -3.267595 & -6.186975 & 0.931134 \\
\hline $\mathrm{C}$ & -2.322412 & -4.333285 & 2.392432 \\
\hline $\mathrm{H}$ & -1.461183 & -5.103527 & 0.599419 \\
\hline $\mathrm{H}$ & -3.334575 & -6.590988 & -0.085429 \\
\hline $\mathrm{H}$ & -2.785336 & -6.940685 & 1.565639 \\
\hline $\mathrm{H}$ & -4.289877 & -6.045468 & 1.301563 \\
\hline
\end{tabular}




\begin{tabular}{|c|c|c|c|}
\hline $\mathrm{H}$ & -1.721747 & -3.419440 & 2.407856 \\
\hline $\mathrm{H}$ & -3.296128 & -4.100746 & 2.839423 \\
\hline $\mathrm{H}$ & -1.833021 & -5.079845 & 3.030488 \\
\hline $\mathrm{C}$ & -2.338357 & -1.855039 & -3.224296 \\
\hline $\mathrm{C}$ & -2.484779 & -0.330257 & -3.379491 \\
\hline $\mathrm{C}$ & -2.857436 & -2.570061 & -4.488977 \\
\hline $\mathrm{H}$ & -1.267581 & -2.064790 & -3.138459 \\
\hline $\mathrm{H}$ & -2.136751 & 0.195493 & -2.486589 \\
\hline $\mathrm{H}$ & -1.889200 & 0.012896 & -4.234177 \\
\hline $\mathrm{H}$ & -3.522866 & -0.034021 & -3.570991 \\
\hline $\mathrm{H}$ & -2.697203 & -3.652097 & -4.437336 \\
\hline $\mathrm{H}$ & -3.932873 & -2.399125 & -4.621773 \\
\hline $\mathrm{H}$ & -2.344090 & -2.195314 & -5.383214 \\
\hline $\mathrm{C}$ & -6.436373 & -1.987188 & -0.241819 \\
\hline $\mathrm{C}$ & -7.434951 & -2.522174 & -1.286670 \\
\hline $\mathrm{C}$ & -6.545355 & -0.456971 & -0.109051 \\
\hline $\mathrm{H}$ & -6.712382 & -2.421983 & 0.728787 \\
\hline $\mathrm{H}$ & -7.395126 & -3.615300 & -1.348794 \\
\hline $\mathrm{H}$ & -8.461027 & -2.230909 & -1.030153 \\
\hline $\mathrm{H}$ & -7.213436 & -2.123728 & -2.284062 \\
\hline $\mathrm{H}$ & -5.847019 & -0.071710 & 0.641231 \\
\hline $\mathrm{H}$ & -6.320642 & 0.043803 & -1.058156 \\
\hline $\mathrm{H}$ & -7.563094 & -0.169335 & 0.183473 \\
\hline $\mathrm{C}$ & -4.096547 & 2.504045 & 1.476957 \\
\hline $\mathrm{C}$ & -3.729271 & 1.614291 & 2.223365 \\
\hline $\mathrm{C}$ & -3.291598 & 0.608990 & 3.133975 \\
\hline $\mathrm{C}$ & -4.166716 & 0.132122 & 4.132154 \\
\hline $\mathrm{C}$ & -1.980067 & 0.101036 & 3.069790 \\
\hline $\mathrm{C}$ & -3.731149 & -0.821754 & 5.046906 \\
\hline $\mathrm{H}$ & -5.178937 & 0.522332 & 4.181911 \\
\hline $\mathrm{C}$ & -1.554086 & -0.852898 & 3.989945 \\
\hline $\mathrm{H}$ & -1.296372 & 0.436483 & 2.298676 \\
\hline $\mathrm{C}$ & -2.424105 & -1.314123 & 4.981069 \\
\hline $\mathrm{H}$ & -4.411399 & -1.180955 & 5.814628 \\
\hline $\mathrm{H}$ & -0.539223 & -1.231111 & 3.915836 \\
\hline $\mathrm{H}$ & -2.087816 & -2.057848 & 5.698645 \\
\hline
\end{tabular}

\section{1a allylboration TS 1 (TS-1Re-A)}

B3LYP Energy $=$

$-3225.109005$

B3LYP Free Energy =

$-3223.960735$

$-3223.525367$

$-3223.516991$

M06-2X Derived Solution Free Energy (toluene) $=$

$1(-306.54)$

B3LYP/6-31G(d) Geometry

$\begin{array}{lrrr}\mathrm{C} & 4.051480 & -1.578031 & 0.660590 \\ \mathrm{C} & 4.372780 & -0.461991 & 1.466070 \\ \mathrm{C} & 5.295000 & 0.473359 & 0.979580 \\ \mathrm{C} & 5.899490 & 0.351099 & -0.271710 \\ \mathrm{C} & 5.549190 & -0.751071 & -1.056790 \\ \mathrm{C} & 4.643750 & -1.722021 & -0.617570 \\ \mathrm{O} & 1.304960 & -1.912371 & -0.159180\end{array}$




\begin{tabular}{|c|c|c|c|}
\hline $\mathrm{P}$ & 0.154440 & -0.862981 & 0.316640 \\
\hline $\mathrm{C}$ & 1.821460 & -2.814691 & 0.772540 \\
\hline $\mathrm{O}$ & -0.354910 & -0.314491 & -1.070980 \\
\hline $\mathrm{O}$ & -1.023270 & -1.834981 & 0.871890 \\
\hline $\mathrm{C}$ & 1.020350 & -3.899101 & 1.169970 \\
\hline $\mathrm{C}$ & 3.154710 & -2.663881 & 1.184060 \\
\hline $\mathrm{C}$ & -1.291940 & -3.125951 & 0.428610 \\
\hline $\mathrm{C}$ & -0.332180 & -4.140371 & 0.603890 \\
\hline $\mathrm{C}$ & 3.665960 & -3.621361 & 2.071600 \\
\hline $\mathrm{C}$ & 1.573660 & -4.818691 & 2.076590 \\
\hline $\mathrm{C}$ & 2.880640 & -4.678121 & 2.530010 \\
\hline $\mathrm{C}$ & -2.587340 & -3.390130 & -0.047720 \\
\hline $\mathrm{C}$ & -0.694270 & -5.449201 & 0.242990 \\
\hline $\mathrm{C}$ & -2.903660 & -4.717410 & -0.369990 \\
\hline $\mathrm{C}$ & -1.966110 & -5.739171 & -0.237150 \\
\hline $\mathrm{C}$ & -3.638840 & -2.327370 & -0.192630 \\
\hline $\mathrm{C}$ & -4.019240 & -1.881310 & -1.482180 \\
\hline $\mathrm{C}$ & -5.072400 & -0.967040 & -1.598070 \\
\hline $\mathrm{C}$ & -5.761300 & -0.474960 & -0.486000 \\
\hline $\mathrm{C}$ & -5.356680 & -0.918650 & 0.773720 \\
\hline $\mathrm{C}$ & -4.313980 & -1.836520 & 0.948420 \\
\hline $\mathrm{O}$ & 0.529700 & 0.127569 & 1.349240 \\
\hline $\mathrm{H}$ & 5.554610 & 1.326829 & 1.601230 \\
\hline $\mathrm{H}$ & 6.006540 & -0.869911 & -2.036220 \\
\hline $\mathrm{H}$ & 4.699030 & -3.530491 & 2.393600 \\
\hline $\mathrm{H}$ & 0.961290 & -5.643881 & 2.427300 \\
\hline $\mathrm{H}$ & 3.292530 & -5.398341 & 3.231270 \\
\hline $\mathrm{H}$ & 0.044830 & -6.238971 & 0.335480 \\
\hline $\mathrm{H}$ & -3.904670 & -4.938550 & -0.728530 \\
\hline $\mathrm{H}$ & -2.226850 & -6.758480 & -0.507240 \\
\hline $\mathrm{H}$ & -5.367190 & -0.635010 & -2.590490 \\
\hline $\mathrm{H}$ & -5.884090 & -0.547950 & 1.650210 \\
\hline $\mathrm{H}$ & -0.138020 & 0.675319 & -1.245220 \\
\hline $\mathrm{C}$ & -2.420419 & 2.831250 & -1.669600 \\
\hline $\mathrm{C}$ & -2.696829 & 2.184010 & -0.415900 \\
\hline $\mathrm{C}$ & -2.911859 & 2.877660 & 0.757010 \\
\hline $\mathrm{O}$ & -0.412299 & 4.109309 & -2.727050 \\
\hline B & -0.745689 & 3.288609 & -1.620740 \\
\hline $\mathrm{O}$ & -0.661259 & 4.070169 & -0.328770 \\
\hline $\mathrm{O}$ & 0.173221 & 2.142259 & -1.636870 \\
\hline $\mathrm{C}$ & -0.857539 & 3.562909 & 0.850100 \\
\hline $\mathrm{C}$ & 1.049051 & 2.269319 & -2.799340 \\
\hline $\mathrm{C}$ & -0.987799 & 4.524539 & 1.968510 \\
\hline $\mathrm{C}$ & -1.125759 & 5.892959 & 1.694780 \\
\hline $\mathrm{C}$ & -0.953919 & 4.085409 & 3.317470 \\
\hline $\mathrm{C}$ & -1.059949 & 5.036359 & 4.348260 \\
\hline $\mathrm{C}$ & -1.228709 & 6.820229 & 2.726790 \\
\hline $\mathrm{C}$ & -1.196619 & 6.390059 & 4.056980 \\
\hline $\mathrm{C}$ & 0.936061 & 3.806989 & -3.118760 \\
\hline $\mathrm{C}$ & 1.899831 & 4.664849 & -2.279080 \\
\hline $\mathrm{C}$ & 1.094141 & 4.164669 & -4.597930 \\
\hline $\mathrm{C}$ & 2.442950 & 1.783889 & -2.404550 \\
\hline $\mathrm{C}$ & 0.481580 & 1.387629 & -3.921610 \\
\hline
\end{tabular}




\begin{tabular}{|c|c|c|c|}
\hline $\mathrm{H}$ & -2.499099 & 2.179410 & -2.542090 \\
\hline $\mathrm{H}$ & -2.953289 & 3.772250 & -1.827290 \\
\hline $\mathrm{H}$ & -2.496030 & 1.115480 & -0.344670 \\
\hline $\mathrm{H}$ & -3.294769 & 3.893470 & 0.719790 \\
\hline $\mathrm{H}$ & -3.046509 & 2.346430 & 1.694310 \\
\hline $\mathrm{H}$ & -0.446329 & 2.577699 & 1.074790 \\
\hline $\mathrm{H}$ & -1.137129 & 6.212919 & 0.658350 \\
\hline $\mathrm{H}$ & -1.030739 & 4.693569 & 5.377630 \\
\hline $\mathrm{H}$ & -1.329519 & 7.877009 & 2.496360 \\
\hline $\mathrm{H}$ & -1.275239 & 7.110099 & 4.866700 \\
\hline $\mathrm{H}$ & 1.834581 & 4.421079 & -1.215310 \\
\hline $\mathrm{H}$ & 1.624681 & 5.717329 & -2.400740 \\
\hline $\mathrm{H}$ & 2.939791 & 4.541499 & -2.601270 \\
\hline $\mathrm{H}$ & 0.988761 & 5.247539 & -4.721500 \\
\hline $\mathrm{H}$ & 0.328901 & 3.682409 & -5.210340 \\
\hline $\mathrm{H}$ & 2.082321 & 3.876019 & -4.975340 \\
\hline $\mathrm{H}$ & 2.424930 & 0.711139 & -2.185800 \\
\hline $\mathrm{H}$ & 2.810951 & 2.298739 & -1.514580 \\
\hline $\mathrm{H}$ & 3.154640 & 1.943969 & -3.222760 \\
\hline $\mathrm{H}$ & -0.512980 & 1.725939 & -4.227270 \\
\hline $\mathrm{H}$ & 0.402510 & 0.353329 & -3.573200 \\
\hline $\mathrm{H}$ & 1.134060 & 1.396839 & -4.801140 \\
\hline $\mathrm{C}$ & -3.984060 & -2.308400 & 2.363660 \\
\hline $\mathrm{C}$ & -5.142770 & -3.132560 & 2.959870 \\
\hline $\mathrm{C}$ & -3.599730 & -1.140610 & 3.290690 \\
\hline $\mathrm{H}$ & -3.116500 & -2.971360 & 2.308870 \\
\hline $\mathrm{H}$ & -5.384980 & -3.992350 & 2.325170 \\
\hline $\mathrm{H}$ & -4.874900 & -3.509090 & 3.954630 \\
\hline $\mathrm{H}$ & -6.052230 & -2.529030 & 3.064810 \\
\hline $\mathrm{H}$ & -2.753980 & -0.579370 & 2.881370 \\
\hline $\mathrm{H}$ & -4.433540 & -0.443210 & 3.434760 \\
\hline $\mathrm{H}$ & -3.313840 & -1.518760 & 4.279940 \\
\hline $\mathrm{C}$ & -3.334000 & -2.375450 & -2.757510 \\
\hline $\mathrm{C}$ & -2.774010 & -1.218260 & -3.605270 \\
\hline $\mathrm{C}$ & -4.277300 & -3.260460 & -3.596370 \\
\hline $\mathrm{H}$ & -2.480770 & -2.993940 & -2.464500 \\
\hline $\mathrm{H}$ & -2.066000 & -0.620760 & -3.024410 \\
\hline $\mathrm{H}$ & -2.247290 & -1.613560 & -4.482370 \\
\hline $\mathrm{H}$ & -3.568950 & -0.557200 & -3.970190 \\
\hline $\mathrm{H}$ & -4.636140 & -4.122290 & -3.022710 \\
\hline $\mathrm{H}$ & -5.156240 & -2.698210 & -3.933670 \\
\hline $\mathrm{H}$ & -3.759760 & -3.637470 & -4.486730 \\
\hline $\mathrm{C}$ & -6.935190 & 0.485530 & -0.632450 \\
\hline $\mathrm{C}$ & -8.137230 & -0.195930 & -1.314950 \\
\hline $\mathrm{C}$ & -6.547930 & 1.781940 & -1.366490 \\
\hline $\mathrm{H}$ & -7.249420 & 0.764670 & 0.382780 \\
\hline $\mathrm{H}$ & -8.444550 & -1.095470 & -0.770400 \\
\hline $\mathrm{H}$ & -8.994790 & 0.486220 & -1.361100 \\
\hline $\mathrm{H}$ & -7.892470 & -0.494550 & -2.341360 \\
\hline $\mathrm{H}$ & -5.714039 & 2.285330 & -0.866560 \\
\hline $\mathrm{H}$ & -6.244500 & 1.581260 & -2.400730 \\
\hline $\mathrm{H}$ & -7.398049 & 2.473970 & -1.399530 \\
\hline $\mathrm{C}$ & 3.776460 & -0.256971 & 2.858790 \\
\hline
\end{tabular}




$\begin{array}{lrrr}\mathrm{C} & 3.204390 & 1.158579 & 3.057090 \\ \mathrm{C} & 4.809170 & -0.584211 & 3.956690 \\ \mathrm{H} & 2.939010 & -0.952601 & 2.969660 \\ \mathrm{H} & 2.452490 & 1.381539 & 2.297000 \\ \mathrm{H} & 2.720370 & 1.231049 & 4.038410 \\ \mathrm{H} & 3.986780 & 1.926459 & 3.022740 \\ \mathrm{H} & 5.176480 & -1.613231 & 3.875560 \\ \mathrm{H} & 5.676980 & 0.083629 & 3.892360 \\ \mathrm{H} & 4.364730 & -0.461181 & 4.951950 \\ \mathrm{C} & 4.362220 & -2.921421 & -1.523440 \\ \mathrm{C} & 5.637610 & -3.749771 & -1.775030 \\ \mathrm{C} & 3.702740 & -2.500051 & -2.850290 \\ \mathrm{H} & 3.654870 & -3.578911 & -1.010260 \\ \mathrm{H} & 6.083800 & -4.085461 & -0.832140 \\ \mathrm{H} & 5.406490 & -4.636851 & -2.377330 \\ \mathrm{H} & 6.396300 & -3.170341 & -2.314180 \\ \mathrm{H} & 2.762230 & -1.970611 & -2.667680 \\ \mathrm{H} & 4.356560 & -1.842141 & -3.435080 \\ \mathrm{H} & 3.483310 & -3.381601 & -3.465000 \\ \mathrm{C} & 6.926360 & 1.372739 & -0.744190 \\ \mathrm{C} & 6.497801 & 2.077249 & -0.894510 \\ \mathrm{C} & 8.323800 & 0.741269 & 0.036840 \\ \mathrm{H} & 6.998211 & 2.142169 & -1.927040 \\ \mathrm{H} & 5.525441 & 2.567249 & -2.331230 \\ \mathrm{H} & 7.232811 & 2.839089 & -2.875360 \\ \mathrm{H} & 6.415670 & 1.366259 & 0.037980 \\ \mathrm{H} & 8.651350 & 0.273499 & -1.675250 \\ \mathrm{H} & 8.326720 & -0.031161 & 3.635990 \\ \mathrm{H} & 9.063990 & 1.500498 & 4.028360 \\ \mathrm{C} & -0.810419 & 2.702529 & \\ \mathrm{C} & -0.677950 & 1.524819 & 0.480849 \\ \mathrm{H} & -0.519710 & & \end{array}$

\section{1a allylboration TS 2 (TS-1Re-B)}

B3LYP Energy =

$-3225.108536$

B3LYP Free Energy =

$-3223.959790$

$-3223.525835$

M06-2X Derived Solution Free Energy $($ DCM $)=$

$-3223.517091$

M06-2X Derived Solution Free Energy (toluene) $=$

$1(-332.82)$

B3LYP/6-31G(d) Geometry

$\begin{array}{lrrr}\mathrm{C} & 4.041830 & -1.591190 & 0.611180 \\ \mathrm{C} & 4.343110 & -0.470970 & 1.418160 \\ \mathrm{C} & 5.257480 & 0.475370 & 0.938300 \\ \mathrm{C} & 5.873090 & 0.359070 & -0.308500 \\ \mathrm{C} & 5.544050 & -0.748910 & -1.094240 \\ \mathrm{C} & 4.647180 & -1.730520 & -0.661020 \\ \mathrm{O} & 1.291820 & -1.904830 & -0.187020 \\ \mathrm{P} & 0.124770 & -0.880650 & 0.297220 \\ \mathrm{C} & 1.813450 & -2.829760 & 0.720480 \\ \mathrm{O} & -0.391890 & -0.323130 & -1.082240 \\ \mathrm{O} & -1.033430 & -1.870330 & 0.851370\end{array}$




\begin{tabular}{|c|c|c|c|}
\hline $\mathrm{C}$ & 1.018940 & -3.927330 & 1.095560 \\
\hline $\mathrm{C}$ & 3.149770 & -2.686030 & 1.124500 \\
\hline $\mathrm{C}$ & -1.301760 & -3.153080 & 0.385150 \\
\hline $\mathrm{C}$ & -0.336010 & -4.165090 & 0.533320 \\
\hline $\mathrm{C}$ & 3.670370 & -3.661670 & 1.986240 \\
\hline $\mathrm{C}$ & 1.581919 & -4.865060 & 1.977580 \\
\hline $\mathrm{C}$ & 2.891069 & -4.730630 & 2.426180 \\
\hline $\mathrm{C}$ & -2.600250 & -3.410350 & -0.084640 \\
\hline $\mathrm{C}$ & -0.696731 & -5.467900 & 0.149690 \\
\hline $\mathrm{C}$ & -2.915231 & -4.732230 & -0.429340 \\
\hline $\mathrm{C}$ & -1.972231 & -5.752500 & -0.324520 \\
\hline $\mathrm{C}$ & -3.650570 & -2.343330 & -0.200990 \\
\hline $\mathrm{C}$ & -4.052080 & -1.885330 & -1.480120 \\
\hline $\mathrm{C}$ & -5.101300 & -0.963299 & -1.569810 \\
\hline $\mathrm{C}$ & -5.764970 & -0.473569 & -0.441470 \\
\hline $\mathrm{C}$ & -5.337780 & -0.927339 & 0.807380 \\
\hline $\mathrm{C}$ & -4.298470 & -1.853610 & 0.956570 \\
\hline $\mathrm{O}$ & 0.483650 & 0.106030 & 1.343220 \\
\hline $\mathrm{H}$ & 5.501680 & 1.332990 & 1.560180 \\
\hline $\mathrm{H}$ & 6.009970 & -0.862510 & -2.070200 \\
\hline $\mathrm{H}$ & 4.706170 & -3.576440 & 2.300800 \\
\hline $\mathrm{H}$ & 0.974729 & -5.700340 & 2.312890 \\
\hline $\mathrm{H}$ & 3.309519 & -5.465420 & 3.108110 \\
\hline $\mathrm{H}$ & 0.045359 & -6.257140 & 0.220010 \\
\hline $\mathrm{H}$ & -3.918581 & -4.950580 & -0.783060 \\
\hline $\mathrm{H}$ & -2.231771 & -6.767360 & -0.611900 \\
\hline $\mathrm{H}$ & -5.412720 & -0.623189 & -2.554390 \\
\hline $\mathrm{H}$ & -5.844930 & -0.557719 & 1.696260 \\
\hline $\mathrm{H}$ & -0.183880 & 0.669050 & -1.263510 \\
\hline $\mathrm{C}$ & -2.401570 & 3.090470 & -1.610370 \\
\hline $\mathrm{C}$ & -2.755430 & 2.230690 & -0.516170 \\
\hline $\mathrm{C}$ & -2.892200 & 2.684930 & 0.781440 \\
\hline $\mathrm{O}$ & -0.236750 & 4.297160 & -2.417900 \\
\hline B & -0.688410 & 3.364300 & -1.452340 \\
\hline $\mathrm{O}$ & -0.578350 & 3.895450 & -0.050220 \\
\hline $\mathrm{O}$ & 0.111640 & 2.137580 & -1.630730 \\
\hline $\mathrm{C}$ & -0.810110 & 3.126550 & 0.971590 \\
\hline $\mathrm{C}$ & 1.006520 & 2.331680 & -2.765810 \\
\hline $\mathrm{C}$ & -0.805120 & 3.702250 & 2.333780 \\
\hline $\mathrm{C}$ & -1.159880 & 5.041560 & 2.641130 \\
\hline $\mathrm{C}$ & -0.398710 & 2.842320 & 3.366080 \\
\hline $\mathrm{C}$ & -0.320490 & 3.288670 & 4.682890 \\
\hline $\mathrm{C}$ & -1.072660 & 5.472640 & 3.977790 \\
\hline $\mathrm{C}$ & -0.655630 & 4.609480 & 4.987580 \\
\hline $\mathrm{C}$ & 1.075190 & 3.902390 & -2.848570 \\
\hline $\mathrm{C}$ & 2.112640 & 4.505760 & -1.884790 \\
\hline $\mathrm{C}$ & 1.302560 & 4.456100 & -4.256600 \\
\hline $\mathrm{C}$ & 2.332440 & 1.636230 & -2.461280 \\
\hline $\mathrm{C}$ & 0.347570 & 1.696620 & -3.999060 \\
\hline $\mathrm{H}$ & -2.520260 & 2.642140 & -2.598490 \\
\hline $\mathrm{H}$ & -2.833300 & 4.093140 & -1.568250 \\
\hline $\mathrm{H}$ & -2.672860 & 1.154820 & -0.668890 \\
\hline $\mathrm{H}$ & -3.173480 & 3.718980 & 0.961710 \\
\hline
\end{tabular}




\begin{tabular}{|c|c|c|c|}
\hline $\mathrm{H}$ & -3.097880 & 1.983690 & 1.585040 \\
\hline $\mathrm{H}$ & -0.461660 & 2.095410 & 0.920950 \\
\hline $\mathrm{H}$ & 0.004980 & 2.609640 & 5.465660 \\
\hline $\mathrm{H}$ & -1.344340 & 6.497820 & 4.208760 \\
\hline $\mathrm{H}$ & -0.595970 & 4.968750 & 6.011190 \\
\hline $\mathrm{H}$ & 1.998260 & 4.111200 & -0.871610 \\
\hline $\mathrm{H}$ & 1.958350 & 5.588400 & -1.841580 \\
\hline $\mathrm{H}$ & 3.138750 & 4.316220 & -2.219790 \\
\hline $\mathrm{H}$ & 1.326810 & 5.550050 & -4.216300 \\
\hline $\mathrm{H}$ & 0.498410 & 4.164700 & -4.936350 \\
\hline $\mathrm{H}$ & 2.257440 & 4.111740 & -4.671300 \\
\hline $\mathrm{H}$ & 2.190380 & 0.551470 & -2.412420 \\
\hline $\mathrm{H}$ & 2.748420 & 1.961280 & -1.505280 \\
\hline $\mathrm{H}$ & 3.065630 & 1.839770 & -3.250090 \\
\hline $\mathrm{H}$ & -0.593290 & 2.196650 & -4.246780 \\
\hline $\mathrm{H}$ & 0.135520 & 0.641690 & -3.796580 \\
\hline $\mathrm{H}$ & 1.005060 & 1.745720 & -4.873690 \\
\hline $\mathrm{C}$ & -3.940630 & -2.330020 & 2.363780 \\
\hline $\mathrm{C}$ & -5.107330 & -3.108639 & 3.004060 \\
\hline $\mathrm{C}$ & -3.483540 & -1.171330 & 3.269890 \\
\hline $\mathrm{H}$ & -3.100040 & -3.024710 & 2.285770 \\
\hline $\mathrm{H}$ & -5.406230 & -3.958799 & 2.380630 \\
\hline $\mathrm{H}$ & -4.816180 & -3.494649 & 3.988510 \\
\hline $\mathrm{H}$ & -5.988820 & -2.471629 & 3.143350 \\
\hline $\mathrm{H}$ & -2.617610 & -0.657830 & 2.840360 \\
\hline $\mathrm{H}$ & -4.282710 & -0.435310 & 3.420770 \\
\hline $\mathrm{H}$ & -3.195820 & -1.551660 & 4.257560 \\
\hline $\mathrm{C}$ & -3.390920 & -2.369620 & -2.771980 \\
\hline $\mathrm{C}$ & -2.822850 & -1.206720 & -3.607040 \\
\hline $\mathrm{C}$ & -4.357340 & -3.226660 & -3.613280 \\
\hline $\mathrm{H}$ & -2.543860 & -3.005930 & -2.500340 \\
\hline $\mathrm{H}$ & -2.094080 & -0.633360 & -3.027110 \\
\hline $\mathrm{H}$ & -2.318460 & -1.595050 & -4.500260 \\
\hline $\mathrm{H}$ & -3.610590 & -0.523380 & -3.945420 \\
\hline $\mathrm{H}$ & -4.725450 & -4.089789 & -3.047410 \\
\hline $\mathrm{H}$ & -5.229770 & -2.645679 & -3.935250 \\
\hline $\mathrm{H}$ & -3.854720 & -3.600550 & -4.513420 \\
\hline $\mathrm{C}$ & -6.939080 & 0.490831 & -0.558110 \\
\hline $\mathrm{C}$ & -8.169750 & -0.200449 & -1.177650 \\
\hline $\mathrm{C}$ & -6.580490 & 1.771921 & -1.331900 \\
\hline $\mathrm{H}$ & -7.210910 & 0.789791 & 0.463680 \\
\hline $\mathrm{H}$ & -8.457460 & -1.087819 & -0.603150 \\
\hline $\mathrm{H}$ & -9.026830 & 0.483391 & -1.203430 \\
\hline $\mathrm{H}$ & -7.966240 & -0.520769 & -2.206540 \\
\hline $\mathrm{H}$ & -5.724350 & 2.282121 & -0.878620 \\
\hline $\mathrm{H}$ & -6.324730 & 1.552401 & -2.375190 \\
\hline $\mathrm{H}$ & -7.429480 & 2.465951 & -1.339550 \\
\hline $\mathrm{C}$ & 3.732830 & -0.278500 & 2.806380 \\
\hline $\mathrm{C}$ & 3.196060 & 1.146740 & 3.028510 \\
\hline $\mathrm{C}$ & 4.740260 & -0.654880 & 3.911910 \\
\hline $\mathrm{H}$ & 2.875140 & -0.953070 & 2.890600 \\
\hline $\mathrm{H}$ & 2.476850 & 1.414850 & 2.251130 \\
\hline $\mathrm{H}$ & 2.685890 & 1.204120 & 3.997680 \\
\hline
\end{tabular}




$\begin{array}{rrrr}\mathrm{H} & 3.998510 & 1.893970 & 3.040770 \\ \mathrm{H} & 5.078100 & -1.692430 & 3.816710 \\ \mathrm{H} & 5.627640 & -0.011360 & 3.870750 \\ \mathrm{H} & 4.285790 & -0.537550 & 4.903380 \\ \mathrm{C} & 4.383690 & -2.932710 & -1.568530 \\ \mathrm{C} & 5.669760 & -3.744530 & -1.818880 \\ \mathrm{C} & 3.721370 & -2.517790 & -2.896000 \\ \mathrm{H} & 3.683600 & -3.599580 & -1.057390 \\ \mathrm{H} & 6.118640 & -4.075800 & -0.875720 \\ \mathrm{H} & 5.450559 & -4.633660 & -2.422570 \\ \mathrm{H} & 6.422070 & -3.155270 & -2.356310 \\ \mathrm{H} & 2.774530 & -1.999340 & -2.714310 \\ \mathrm{H} & 4.368230 & -1.851190 & -3.478620 \\ \mathrm{H} & 3.513530 & -3.401110 & -3.512180 \\ \mathrm{C} & 6.886300 & 1.396100 & -0.776980 \\ \mathrm{C} & 6.446480 & 2.098510 & -2.074820 \\ \mathrm{C} & 8.292440 & 0.785129 & -0.932410 \\ \mathrm{H} & 6.947340 & 2.163890 & 0.006500 \\ \mathrm{H} & 5.467380 & 2.574470 & -1.954490 \\ \mathrm{H} & 7.170140 & 2.871600 & -2.360060 \\ \mathrm{H} & 6.373200 & 1.388790 & -2.907580 \\ \mathrm{H} & 8.627260 & 0.319129 & -1.713320 \\ \mathrm{H} & 8.306360 & 0.015429 & -1.210780 \\ \mathrm{H} & 9.021410 & 1.555859 & 1.663450 \\ \mathrm{C} & -1.637830 & 5.967430 & 0.905540 \\ \mathrm{C} & -2.079340 & 6.801310 & 0.198350 \\ \mathrm{H} & -2.434410 & 7.516130 & 3.114680 \\ \mathrm{H} & -0.127410 & 1.820310 & \\ & & & \end{array}$

\section{1d allylboration TS 2 (TS-3Re-B)}

B3LYP Energy =

$-3456.181120$

B3LYP Free Energy =

$-3454.953936$

$-3454.472979$

M06-2X Derived Solution Free Energy $(\mathrm{DCM})=$

M06-2X Derived Solution Free Energy (toluene) $=$

$-3454.464439$

Number of Imaginary Frequencies $=$

$1(-352.17)$

B3LYP/6-31G(d) Geometry

$\begin{array}{lrrr}\mathrm{C} & -4.438190 & -1.888612 & 0.443450 \\ \mathrm{C} & -3.860969 & -2.784171 & 1.371870 \\ \mathrm{C} & -3.633319 & -4.108151 & 0.974830 \\ \mathrm{C} & -3.946469 & -4.572551 & -0.302870 \\ \mathrm{C} & -4.505679 & -3.663492 & -1.205640 \\ \mathrm{C} & -4.764289 & -2.333312 & -0.860770 \\ \mathrm{O} & -2.915990 & 0.418659 & -0.357380 \\ \mathrm{P} & -1.429480 & 0.705979 & 0.233750 \\ \mathrm{C} & -4.032550 & 0.628869 & 0.455430 \\ \mathrm{O} & -0.567630 & 0.712400 & -1.085900 \\ \mathrm{O} & -1.518301 & 2.247599 & 0.728570 \\ \mathrm{C} & -4.418661 & 1.950068 & 0.739860 \\ \mathrm{C} & -4.786180 & -0.486222 & 0.853820 \\ \mathrm{C} & -2.303181 & 3.243419 & 0.156190 \\ \mathrm{C} & -3.705271 & 3.130749 & 0.187240\end{array}$




\begin{tabular}{|c|c|c|c|}
\hline $\mathrm{C}$ & -5.940200 & -0.244922 & 1.612310 \\
\hline $\mathrm{C}$ & -5.572361 & 2.135678 & 1.520110 \\
\hline $\mathrm{C}$ & -6.322351 & 1.050228 & 1.959000 \\
\hline $\mathrm{C}$ & -1.647902 & 4.398209 & -0.301920 \\
\hline $\mathrm{C}$ & -4.454782 & 4.212618 & -0.305070 \\
\hline $\mathrm{C}$ & -2.444292 & 5.457239 & -0.759870 \\
\hline $\mathrm{C}$ & -3.834432 & 5.365529 & -0.772800 \\
\hline $\mathrm{C}$ & -0.153602 & 4.546190 & -0.289950 \\
\hline $\mathrm{C}$ & 0.565798 & 4.536410 & -1.510590 \\
\hline $\mathrm{C}$ & 1.943968 & 4.778501 & -1.485570 \\
\hline $\mathrm{C}$ & 2.639468 & 5.023481 & -0.298290 \\
\hline $\mathrm{C}$ & 1.910508 & 5.007881 & 0.891740 \\
\hline $\mathrm{C}$ & 0.529248 & 4.780760 & 0.925960 \\
\hline $\mathrm{O}$ & -0.965370 & -0.151800 & 1.349200 \\
\hline $\mathrm{H}$ & -3.198448 & -4.804641 & 1.687900 \\
\hline $\mathrm{H}$ & -4.764259 & -4.002422 & -2.206170 \\
\hline $\mathrm{H}$ & -6.543340 & -1.092832 & 1.923040 \\
\hline $\mathrm{H}$ & -5.869741 & 3.145858 & 1.784430 \\
\hline $\mathrm{H}$ & -7.211131 & 1.212567 & 2.562130 \\
\hline $\mathrm{H}$ & -5.537232 & 4.131738 & -0.324510 \\
\hline $\mathrm{H}$ & -1.953783 & 6.361729 & -1.107300 \\
\hline $\mathrm{H}$ & -4.432522 & 6.191928 & -1.145980 \\
\hline $\mathrm{H}$ & 2.487918 & 4.783331 & -2.426790 \\
\hline $\mathrm{H}$ & 2.435068 & 5.198971 & 1.825480 \\
\hline $\mathrm{H}$ & 0.098740 & -0.063460 & -1.180850 \\
\hline $\mathrm{C}$ & 3.382880 & 0.233121 & -1.186370 \\
\hline $\mathrm{C}$ & 2.824109 & 1.038821 & -0.139820 \\
\hline $\mathrm{C}$ & 3.151189 & 0.863101 & 1.192800 \\
\hline $\mathrm{O}$ & 3.130701 & -2.233059 & -1.987510 \\
\hline B & 2.548030 & -1.297999 & -1.086830 \\
\hline $\mathrm{O}$ & 2.754680 & -1.693579 & 0.339440 \\
\hline $\mathrm{O}$ & 1.120820 & -1.204030 & -1.433010 \\
\hline $\mathrm{C}$ & 2.215010 & -1.024769 & 1.319590 \\
\hline $\mathrm{C}$ & 0.863741 & -2.054310 & -2.588240 \\
\hline $\mathrm{C}$ & 2.560060 & -1.376579 & 2.713380 \\
\hline $\mathrm{C}$ & 3.791480 & -1.968209 & 3.108140 \\
\hline $\mathrm{C}$ & 1.587180 & -1.091569 & 3.684140 \\
\hline $\mathrm{C}$ & 1.803470 & -1.385949 & 5.027370 \\
\hline $\mathrm{C}$ & 3.989241 & -2.254829 & 4.474130 \\
\hline $\mathrm{C}$ & 3.010720 & -1.969449 & 5.420940 \\
\hline $\mathrm{C}$ & 2.080371 & -3.053679 & -2.527840 \\
\hline $\mathrm{C}$ & 1.836831 & -4.235129 & -1.573130 \\
\hline $\mathrm{C}$ & 2.537681 & -3.579729 & -3.889530 \\
\hline $\mathrm{C}$ & -0.512749 & -2.696020 & -2.418140 \\
\hline $\mathrm{C}$ & 0.881080 & -1.165280 & -3.839910 \\
\hline $\mathrm{H}$ & 3.184130 & 0.593551 & -2.197140 \\
\hline $\mathrm{H}$ & 4.433750 & -0.033418 & -1.051550 \\
\hline $\mathrm{H}$ & 1.939629 & 1.632011 & -0.370950 \\
\hline $\mathrm{H}$ & 4.125440 & 0.459062 & 1.455470 \\
\hline $\mathrm{H}$ & 2.659249 & 1.459321 & 1.955780 \\
\hline $\mathrm{H}$ & 1.193310 & -0.668680 & 1.186540 \\
\hline $\mathrm{H}$ & 1.034120 & -1.164950 & 5.761470 \\
\hline $\mathrm{H}$ & 4.929051 & -2.707078 & 4.775060 \\
\hline
\end{tabular}




\begin{tabular}{|c|c|c|c|}
\hline $\mathrm{H}$ & 3.190561 & -2.203889 & 6.466590 \\
\hline $\mathrm{H}$ & 1.496111 & -3.894949 & -0.591750 \\
\hline $\mathrm{H}$ & 2.780322 & -4.771789 & -1.431890 \\
\hline $\mathrm{H}$ & 1.098892 & -4.938150 & -1.975720 \\
\hline $\mathrm{H}$ & 3.392511 & -4.250229 & -3.751830 \\
\hline $\mathrm{H}$ & 2.851611 & -2.767129 & -4.549060 \\
\hline $\mathrm{H}$ & 1.740971 & -4.147609 & -4.384390 \\
\hline $\mathrm{H}$ & -1.294990 & -1.930720 & -2.458780 \\
\hline $\mathrm{H}$ & -0.601199 & -3.212450 & -1.459890 \\
\hline $\mathrm{H}$ & -0.708589 & -3.414280 & -3.222680 \\
\hline $\mathrm{H}$ & 1.866030 & -0.714209 & -3.991660 \\
\hline $\mathrm{H}$ & 0.147270 & -0.361050 & -3.724230 \\
\hline $\mathrm{H}$ & 0.618790 & -1.733550 & -4.738690 \\
\hline $\mathrm{C}$ & -0.187512 & 4.845650 & 2.274600 \\
\hline $\mathrm{C}$ & -0.095752 & 6.256000 & 2.890560 \\
\hline $\mathrm{C}$ & 0.329428 & 3.776880 & 3.256190 \\
\hline $\mathrm{H}$ & -1.248562 & 4.641020 & 2.108270 \\
\hline $\mathrm{H}$ & -0.503493 & 7.012780 & 2.211020 \\
\hline $\mathrm{H}$ & -0.661463 & 6.301280 & 3.829000 \\
\hline $\mathrm{H}$ & 0.941537 & 6.532360 & 3.113280 \\
\hline $\mathrm{H}$ & 0.206159 & 2.772340 & 2.839540 \\
\hline $\mathrm{H}$ & 1.389698 & 3.926071 & 3.494250 \\
\hline $\mathrm{H}$ & -0.229382 & 3.823260 & 4.198810 \\
\hline $\mathrm{C}$ & -0.106402 & 4.285890 & -2.861880 \\
\hline $\mathrm{C}$ & 0.514989 & 3.089330 & -3.606740 \\
\hline $\mathrm{C}$ & -0.085872 & 5.549130 & -3.745530 \\
\hline $\mathrm{H}$ & -1.154792 & 4.034140 & -2.678020 \\
\hline $\mathrm{H}$ & 0.457329 & 2.181980 & -2.998770 \\
\hline $\mathrm{H}$ & -0.024031 & 2.906400 & -4.544360 \\
\hline $\mathrm{H}$ & 1.566289 & 3.268431 & -3.861860 \\
\hline $\mathrm{H}$ & -0.568643 & 6.397140 & -3.247030 \\
\hline $\mathrm{H}$ & 0.940188 & 5.849510 & -3.989450 \\
\hline $\mathrm{H}$ & -0.613182 & 5.365840 & -4.689470 \\
\hline $\mathrm{C}$ & 4.130158 & 5.338582 & -0.293240 \\
\hline $\mathrm{C}$ & 4.415517 & 6.700382 & -0.956810 \\
\hline $\mathrm{C}$ & 4.974548 & 4.225052 & -0.938200 \\
\hline $\mathrm{H}$ & 4.438098 & 5.416302 & 0.758640 \\
\hline $\mathrm{H}$ & 3.847767 & 7.503401 & -0.474090 \\
\hline $\mathrm{H}$ & 5.481957 & 6.947632 & -0.890990 \\
\hline $\mathrm{H}$ & 4.140237 & 6.688592 & -2.018270 \\
\hline $\mathrm{H}$ & 4.802939 & 3.261762 & -0.446910 \\
\hline $\mathrm{H}$ & 4.735268 & 4.105192 & -2.001440 \\
\hline $\mathrm{H}$ & 6.042808 & 4.461642 & -0.865030 \\
\hline $\mathrm{C}$ & -3.505799 & -2.369141 & 2.799550 \\
\hline $\mathrm{C}$ & -2.067349 & -2.756531 & 3.187790 \\
\hline $\mathrm{C}$ & -4.515849 & -2.946042 & 3.812170 \\
\hline $\mathrm{H}$ & -3.564050 & -1.277901 & 2.856160 \\
\hline $\mathrm{H}$ & -1.350029 & -2.331090 & 2.482300 \\
\hline $\mathrm{H}$ & -1.835009 & -2.368831 & 4.187290 \\
\hline $\mathrm{H}$ & -1.928459 & -3.843651 & 3.221120 \\
\hline $\mathrm{H}$ & -5.540229 & -2.626802 & 3.590310 \\
\hline $\mathrm{H}$ & -4.500779 & -4.042732 & 3.802180 \\
\hline $\mathrm{H}$ & -4.271869 & -2.616622 & 4.829590 \\
\hline
\end{tabular}




\begin{tabular}{|c|c|c|c|}
\hline $\mathrm{C}$ & -5.429010 & -1.424742 & -1.895650 \\
\hline $\mathrm{C}$ & -6.834990 & -1.933802 & -2.270120 \\
\hline $\mathrm{C}$ & -4.553790 & -1.236962 & -3.149000 \\
\hline $\mathrm{H}$ & -5.560970 & -0.435952 & -1.447360 \\
\hline $\mathrm{H}$ & -7.472129 & -2.024283 & -1.383160 \\
\hline $\mathrm{H}$ & -7.320260 & -1.241193 & -2.968560 \\
\hline $\mathrm{H}$ & -6.793979 & -2.917812 & -2.751760 \\
\hline $\mathrm{H}$ & -3.580240 & -0.812401 & -2.884310 \\
\hline $\mathrm{H}$ & -4.381939 & -2.187292 & -3.668330 \\
\hline $\mathrm{H}$ & -5.041360 & -0.555612 & -3.857020 \\
\hline $\mathrm{C}$ & -3.705358 & -6.028551 & -0.681710 \\
\hline $\mathrm{C}$ & -2.723788 & -6.170741 & -1.859580 \\
\hline $\mathrm{C}$ & -5.026578 & -6.765392 & -0.974900 \\
\hline $\mathrm{H}$ & -3.244788 & -6.515191 & 0.188960 \\
\hline $\mathrm{H}$ & -1.766318 & -5.687211 & -1.637470 \\
\hline $\mathrm{H}$ & -2.530638 & -7.228371 & -2.076090 \\
\hline $\mathrm{H}$ & -3.125688 & -5.713031 & -2.771360 \\
\hline $\mathrm{H}$ & -5.713138 & -6.700992 & -0.123640 \\
\hline $\mathrm{H}$ & -5.535088 & -6.336572 & -1.846620 \\
\hline $\mathrm{H}$ & -4.840907 & -7.825632 & -1.185230 \\
\hline $\mathrm{C}$ & 4.835251 & -2.266518 & 2.188810 \\
\hline $\mathrm{C}$ & 5.755751 & -2.542408 & 1.442500 \\
\hline $\mathrm{H}$ & 0.649200 & -0.645450 & 3.363410 \\
\hline $\mathrm{C}$ & 6.772921 & -2.865298 & 0.498240 \\
\hline $\mathrm{C}$ & 8.102341 & -3.078327 & 0.913870 \\
\hline $\mathrm{C}$ & 6.452581 & -2.975418 & -0.871860 \\
\hline $\mathrm{C}$ & 9.088261 & -3.390577 & -0.018040 \\
\hline $\mathrm{H}$ & 8.347671 & -2.994267 & 1.968390 \\
\hline $\mathrm{C}$ & 7.448781 & -3.288597 & -1.793300 \\
\hline $\mathrm{H}$ & 5.427081 & -2.820378 & -1.196830 \\
\hline $\mathrm{C}$ & 8.765501 & -3.496187 & -1.373450 \\
\hline $\mathrm{H}$ & 10.110631 & -3.552026 & 0.313290 \\
\hline $\mathrm{H}$ & 7.194411 & -3.371177 & -2.846680 \\
\hline $\mathrm{H}$ & 9.537181 & -3.740017 & -2.098850 \\
\hline
\end{tabular}

\section{1d allylboration TS 3 (TS-3Re-A)}

B3LYP Energy $=$

$-3456.178903$

B3LYP Free Energy =

$-3454.952101$

M06-2X Derived Solution Free Energy $($ DCM $)=$

$-3454.475364$

M06-2X Derived Solution Free Energy (toluene) $=$

$-3454.466470$

Number of Imaginary Frequencies $=$

$1(-310.97)$

B3LYP/6-31G(d) Geometry

$\begin{array}{lrrr}\mathrm{C} & 4.069651 & -1.550057 & 0.046969 \\ \mathrm{C} & 4.434330 & -0.548177 & 0.975319 \\ \mathrm{C} & 5.357340 & 0.428884 & 0.580909 \\ \mathrm{C} & 5.924140 & 0.456204 & -0.693061 \\ \mathrm{C} & 5.534730 & -0.536146 & -1.597211 \\ \mathrm{C} & 4.623981 & -1.540827 & -1.256491 \\ \mathrm{O} & 1.334801 & -1.793519 & -0.831291 \\ \mathrm{P} & 0.192760 & -0.773490 & -0.280451 \\ \mathrm{C} & 1.832522 & -2.777039 & 0.025359\end{array}$




\begin{tabular}{|c|c|c|c|}
\hline $\mathrm{O}$ & -0.288300 & -0.087000 & -1.610051 \\
\hline $\mathrm{O}$ & -0.999689 & -1.776330 & 0.181929 \\
\hline $\mathrm{C}$ & 1.015533 & -3.881419 & 0.323819 \\
\hline $\mathrm{C}$ & 3.163042 & -2.673858 & 0.460639 \\
\hline $\mathrm{C}$ & -1.278668 & -3.020681 & -0.374781 \\
\hline $\mathrm{C}$ & -0.333557 & -4.058830 & -0.273241 \\
\hline $\mathrm{C}$ & 3.655642 & -3.708057 & 1.269239 \\
\hline $\mathrm{C}$ & 1.551233 & -4.880439 & 1.154859 \\
\hline $\mathrm{C}$ & 2.855533 & -4.791248 & 1.630469 \\
\hline $\mathrm{C}$ & -2.568188 & -3.225612 & -0.893581 \\
\hline $\mathrm{C}$ & -0.706167 & -5.328500 & -0.745841 \\
\hline $\mathrm{C}$ & -2.895697 & -4.517462 & -1.329381 \\
\hline $\mathrm{C}$ & -1.974086 & -5.560181 & -1.266191 \\
\hline $\mathrm{C}$ & -3.600109 & -2.137412 & -0.980561 \\
\hline $\mathrm{C}$ & -3.927809 & -1.577142 & -2.240071 \\
\hline $\mathrm{C}$ & -4.964430 & -0.640193 & -2.313971 \\
\hline $\mathrm{C}$ & -5.686360 & -0.234124 & -1.188061 \\
\hline $\mathrm{C}$ & -5.332980 & -0.789823 & 0.042249 \\
\hline $\mathrm{C}$ & -4.309119 & -1.735793 & 0.174309 \\
\hline $\mathrm{O}$ & 0.568450 & 0.112561 & 0.846099 \\
\hline $\mathrm{H}$ & 5.646439 & 1.195524 & 1.295859 \\
\hline $\mathrm{H}$ & 5.963890 & -0.540436 & -2.596301 \\
\hline $\mathrm{H}$ & 4.684942 & -3.653717 & 1.610619 \\
\hline $\mathrm{H}$ & 0.928684 & -5.728129 & 1.424909 \\
\hline $\mathrm{H}$ & 3.254424 & -5.572498 & 2.271289 \\
\hline $\mathrm{H}$ & 0.021814 & -6.133140 & -0.710111 \\
\hline $\mathrm{H}$ & -3.892717 & -4.693722 & -1.722141 \\
\hline $\mathrm{H}$ & -2.243466 & -6.549951 & -1.623511 \\
\hline $\mathrm{H}$ & -5.220440 & -0.221903 & -3.284271 \\
\hline $\mathrm{H}$ & -5.887130 & -0.487384 & 0.928189 \\
\hline $\mathrm{H}$ & -0.089861 & 0.930350 & -1.641061 \\
\hline $\mathrm{C}$ & -2.402162 & 3.003199 & -1.489691 \\
\hline $\mathrm{C}$ & -2.634902 & 2.196338 & -0.322231 \\
\hline $\mathrm{C}$ & -2.812312 & 2.734128 & 0.935799 \\
\hline $\mathrm{O}$ & -0.500293 & 4.514790 & -2.408301 \\
\hline B & -0.737633 & 3.492690 & -1.452221 \\
\hline $\mathrm{O}$ & -0.576813 & 4.034760 & -0.054781 \\
\hline $\mathrm{O}$ & 0.207128 & 2.405440 & -1.749021 \\
\hline $\mathrm{C}$ & -0.766413 & 3.410490 & 1.069809 \\
\hline $\mathrm{C}$ & 1.001858 & 2.780191 & -2.915931 \\
\hline $\mathrm{C}$ & -0.879123 & 4.278360 & 2.265929 \\
\hline $\mathrm{C}$ & -1.009714 & 5.664660 & 2.082659 \\
\hline $\mathrm{C}$ & -0.847733 & 3.753660 & 3.584779 \\
\hline $\mathrm{C}$ & -0.949843 & 4.649050 & 4.671329 \\
\hline $\mathrm{C}$ & -1.107685 & 6.529329 & 3.166209 \\
\hline $\mathrm{C}$ & -1.078384 & 6.016989 & 4.467609 \\
\hline $\mathrm{C}$ & 0.825037 & 4.344981 & -2.931951 \\
\hline $\mathrm{C}$ & 1.820566 & 5.069141 & -2.007361 \\
\hline $\mathrm{C}$ & 0.872296 & 4.978051 & -4.324151 \\
\hline $\mathrm{C}$ & 2.431058 & 2.284722 & -2.700611 \\
\hline $\mathrm{C}$ & 0.388428 & 2.101740 & -4.149301 \\
\hline $\mathrm{H}$ & -2.511792 & 2.468938 & -2.435691 \\
\hline $\mathrm{H}$ & -2.947893 & 3.950128 & -1.504181 \\
\hline
\end{tabular}




\begin{tabular}{|c|c|c|c|}
\hline $\mathrm{H}$ & -2.438361 & 1.127569 & -0.398771 \\
\hline $\mathrm{H}$ & -3.202303 & 3.742608 & 1.038139 \\
\hline $\mathrm{H}$ & -2.919492 & 2.088288 & 1.802439 \\
\hline $\mathrm{H}$ & -0.359342 & 2.405160 & 1.194929 \\
\hline $\mathrm{H}$ & -1.019874 & 6.047489 & 1.068229 \\
\hline $\mathrm{H}$ & -0.923153 & 4.242730 & 5.677299 \\
\hline $\mathrm{H}$ & -1.202445 & 7.598539 & 2.999549 \\
\hline $\mathrm{H}$ & -1.152865 & 6.684239 & 5.321889 \\
\hline $\mathrm{H}$ & 1.825347 & 4.638041 & -1.002691 \\
\hline $\mathrm{H}$ & 1.516106 & 6.117131 & -1.921411 \\
\hline $\mathrm{H}$ & 2.841166 & 5.038972 & -2.404921 \\
\hline $\mathrm{H}$ & 0.725476 & 6.059401 & -4.234211 \\
\hline $\mathrm{H}$ & 0.082767 & 4.586730 & -4.969711 \\
\hline $\mathrm{H}$ & 1.841257 & 4.807841 & -4.808341 \\
\hline $\mathrm{H}$ & 2.456989 & 1.189862 & -2.688251 \\
\hline $\mathrm{H}$ & 2.840138 & 2.636752 & -1.751261 \\
\hline $\mathrm{H}$ & 3.084118 & 2.624212 & -3.513211 \\
\hline $\mathrm{H}$ & -0.633162 & 2.451110 & -4.325741 \\
\hline $\mathrm{H}$ & 0.362749 & 1.017900 & -4.000411 \\
\hline $\mathrm{H}$ & 0.981338 & 2.301991 & -5.048001 \\
\hline $\mathrm{C}$ & -4.037179 & -2.332523 & 1.554209 \\
\hline $\mathrm{C}$ & -5.221748 & -3.198313 & 2.027629 \\
\hline $\mathrm{C}$ & -3.687569 & -1.254662 & 2.596309 \\
\hline $\mathrm{H}$ & -3.169738 & -2.993672 & 1.475909 \\
\hline $\mathrm{H}$ & -5.439117 & -3.998584 & 1.311179 \\
\hline $\mathrm{H}$ & -4.996598 & -3.660023 & 2.996599 \\
\hline $\mathrm{H}$ & -6.132878 & -2.599794 & 2.146009 \\
\hline $\mathrm{H}$ & -2.822910 & -0.665832 & 2.275129 \\
\hline $\mathrm{H}$ & -4.525970 & -0.568833 & 2.768839 \\
\hline $\mathrm{H}$ & -3.436399 & -1.718762 & 3.556839 \\
\hline $\mathrm{C}$ & -3.206339 & -1.976622 & -3.528451 \\
\hline $\mathrm{C}$ & -2.623880 & -0.762212 & -4.274921 \\
\hline $\mathrm{C}$ & -4.125488 & -2.797293 & -4.454871 \\
\hline $\mathrm{H}$ & -2.360478 & -2.615351 & -3.257981 \\
\hline $\mathrm{H}$ & -1.939480 & -0.204621 & -3.629941 \\
\hline $\mathrm{H}$ & -2.065769 & -1.095141 & -5.158431 \\
\hline $\mathrm{H}$ & -3.408190 & -0.078872 & -4.621401 \\
\hline $\mathrm{H}$ & -4.500998 & -3.697113 & -3.955131 \\
\hline $\mathrm{H}$ & -4.993999 & -2.209483 & -4.775231 \\
\hline $\mathrm{H}$ & -3.583178 & -3.110922 & -5.355091 \\
\hline $\mathrm{C}$ & -6.840951 & 0.754646 & -1.291331 \\
\hline $\mathrm{C}$ & -8.022700 & 0.158755 & -2.081031 \\
\hline $\mathrm{C}$ & -6.407182 & 2.106536 & -1.886051 \\
\hline $\mathrm{H}$ & -7.193311 & 0.944275 & -0.268021 \\
\hline $\mathrm{H}$ & -8.361750 & -0.783146 & -1.636011 \\
\hline $\mathrm{H}$ & -8.869741 & 0.855314 & -2.095531 \\
\hline $\mathrm{H}$ & -7.741190 & -0.046005 & -3.120971 \\
\hline $\mathrm{H}$ & -5.590972 & 2.550016 & -1.306421 \\
\hline $\mathrm{H}$ & -6.060662 & 1.996156 & -2.920421 \\
\hline $\mathrm{H}$ & -7.246992 & 2.811815 & -1.892151 \\
\hline $\mathrm{C}$ & 3.878830 & -0.499127 & 2.398679 \\
\hline $\mathrm{C}$ & 3.271649 & 0.872842 & 2.745919 \\
\hline $\mathrm{C}$ & 4.953260 & -0.897036 & 3.430929 \\
\hline
\end{tabular}




\begin{tabular}{|c|c|c|c|}
\hline $\mathrm{H}$ & 3.067291 & -1.229248 & 2.471149 \\
\hline $\mathrm{H}$ & 2.492849 & 1.142382 & 2.028749 \\
\hline $\mathrm{H}$ & 2.816299 & 0.837222 & 3.742519 \\
\hline $\mathrm{H}$ & 4.030369 & 1.664633 & 2.759189 \\
\hline $\mathrm{H}$ & 5.354881 & -1.897606 & 3.233889 \\
\hline $\mathrm{H}$ & 5.795460 & -0.194396 & 3.417449 \\
\hline $\mathrm{H}$ & 4.531110 & -0.894727 & 4.443059 \\
\hline $\mathrm{C}$ & 4.297432 & -2.611517 & -2.298331 \\
\hline $\mathrm{C}$ & 5.546722 & -3.434246 & -2.671661 \\
\hline $\mathrm{C}$ & 3.633671 & -2.012417 & -3.552731 \\
\hline $\mathrm{H}$ & 3.580002 & -3.310447 & -1.859531 \\
\hline $\mathrm{H}$ & 5.997883 & -3.894156 & -1.785231 \\
\hline $\mathrm{H}$ & 5.282783 & -4.234796 & -3.373361 \\
\hline $\mathrm{H}$ & 6.312232 & -2.811905 & -3.150011 \\
\hline $\mathrm{H}$ & 2.715151 & -1.477778 & -3.290991 \\
\hline $\mathrm{H}$ & 4.302591 & -1.312417 & -4.067641 \\
\hline $\mathrm{H}$ & 3.374212 & -2.806337 & -4.263931 \\
\hline $\mathrm{C}$ & 6.950429 & 1.519105 & -1.065621 \\
\hline $\mathrm{C}$ & 6.501848 & 2.372615 & -2.265981 \\
\hline $\mathrm{C}$ & 8.338559 & 0.899986 & -1.319641 \\
\hline $\mathrm{H}$ & 7.044008 & 2.191655 & -0.201931 \\
\hline $\mathrm{H}$ & 5.534448 & 2.848704 & -2.073881 \\
\hline $\mathrm{H}$ & 7.235738 & 3.160445 & -2.474721 \\
\hline $\mathrm{H}$ & 6.400859 & 1.764865 & -3.173091 \\
\hline $\mathrm{H}$ & 8.682170 & 0.328436 & -0.450511 \\
\hline $\mathrm{H}$ & 8.317400 & 0.219136 & -2.179001 \\
\hline $\mathrm{H}$ & 9.079449 & 1.680796 & -1.530371 \\
\hline $\mathrm{C}$ & -0.724982 & 2.366350 & 3.877349 \\
\hline $\mathrm{C}$ & -0.614621 & 1.215950 & 4.260389 \\
\hline $\mathrm{C}$ & -0.480730 & -0.120860 & 4.737109 \\
\hline $\mathrm{C}$ & -0.039609 & -1.149980 & 3.881079 \\
\hline $\mathrm{C}$ & -0.785430 & -0.423500 & 6.080119 \\
\hline $\mathrm{C}$ & 0.090912 & -2.449060 & 4.367049 \\
\hline $\mathrm{H}$ & 0.193640 & -0.910620 & 2.848939 \\
\hline $\mathrm{C}$ & -0.653109 & -1.726000 & 6.552649 \\
\hline $\mathrm{H}$ & -1.125090 & 0.369889 & 6.739229 \\
\hline $\mathrm{C}$ & -0.214358 & -2.741930 & 5.698849 \\
\hline $\mathrm{H}$ & 0.434472 & -3.235160 & 3.699899 \\
\hline $\mathrm{H}$ & -0.891649 & -1.949230 & 7.589169 \\
\hline $\mathrm{H}$ & -0.110608 & -3.757600 & 6.071609 \\
\hline
\end{tabular}

\section{1d propargylboration TS 1}

B3LYP Energy =

$-3454.928914$

B3LYP Free Energy =

$-3453.726398$

M06-2X Derived Solution Free Energy (toluene) =

$-3453.241214$

$1(-365.93)$

B3LYP/6-31G(d) Geometry

$\begin{array}{lrrr}\mathrm{C} & -4.559421 & -1.626638 & 0.455380 \\ \mathrm{C} & -4.054381 & -2.514169 & 1.432000 \\ \mathrm{C} & -3.906232 & -3.866279 & 1.097830 \\ \mathrm{C} & -4.231132 & -4.367269 & -0.163000\end{array}$




\begin{tabular}{|c|c|c|c|}
\hline $\mathrm{C}$ & -4.716621 & -3.466118 & -1.115050 \\
\hline $\mathrm{C}$ & -4.893481 & -2.107918 & -0.833660 \\
\hline $\mathrm{O}$ & -2.876060 & 0.544011 & -0.398380 \\
\hline $\mathrm{P}$ & -1.378580 & 0.770550 & 0.192980 \\
\hline $\mathrm{C}$ & -3.992760 & 0.858501 & 0.377440 \\
\hline $\mathrm{O}$ & -0.519550 & 0.701220 & -1.125610 \\
\hline $\mathrm{O}$ & -1.393629 & 2.323460 & 0.659300 \\
\hline $\mathrm{C}$ & -4.302719 & 2.212031 & 0.595720 \\
\hline $\mathrm{C}$ & -4.826200 & -0.189038 & 0.799100 \\
\hline $\mathrm{C}$ & -2.094909 & 3.349751 & 0.034800 \\
\hline $\mathrm{C}$ & -3.501279 & 3.322771 & 0.018980 \\
\hline $\mathrm{C}$ & -5.981890 & 0.155112 & 1.514140 \\
\hline $\mathrm{C}$ & -5.461899 & 2.501822 & 1.335030 \\
\hline $\mathrm{C}$ & -6.290420 & 1.484962 & 1.796530 \\
\hline $\mathrm{C}$ & -1.354598 & 4.450130 & -0.427200 \\
\hline $\mathrm{C}$ & -4.165568 & 4.428061 & -0.539380 \\
\hline $\mathrm{C}$ & -2.068008 & 5.536661 & -0.952530 \\
\hline $\mathrm{C}$ & -3.459578 & 5.524101 & -1.022260 \\
\hline $\mathrm{C}$ & 0.142842 & 4.520690 & -0.337810 \\
\hline $\mathrm{C}$ & 0.927652 & 4.420539 & -1.512020 \\
\hline $\mathrm{C}$ & 2.311632 & 4.603429 & -1.418490 \\
\hline $\mathrm{C}$ & 2.950682 & 4.866369 & -0.204220 \\
\hline $\mathrm{C}$ & 2.157492 & 4.935039 & 0.942050 \\
\hline $\mathrm{C}$ & 0.766952 & 4.775030 & 0.905300 \\
\hline $\mathrm{O}$ & -0.959750 & -0.081840 & 1.330540 \\
\hline $\mathrm{H}$ & -3.527582 & -4.555639 & 1.848850 \\
\hline $\mathrm{H}$ & -4.982232 & -3.832528 & -2.103980 \\
\hline $\mathrm{H}$ & -6.645000 & -0.640068 & 1.841650 \\
\hline $\mathrm{H}$ & -5.701079 & 3.538852 & 1.549650 \\
\hline $\mathrm{H}$ & -7.182249 & 1.727893 & 2.367200 \\
\hline $\mathrm{H}$ & -5.249528 & 4.411222 & -0.597680 \\
\hline $\mathrm{H}$ & -1.511848 & 6.400810 & -1.303470 \\
\hline $\mathrm{H}$ & -3.992348 & 6.369691 & -1.447770 \\
\hline $\mathrm{H}$ & 2.906582 & 4.540849 & -2.326130 \\
\hline $\mathrm{H}$ & 2.638642 & 5.140869 & 1.896100 \\
\hline $\mathrm{H}$ & 0.089120 & -0.116350 & -1.205070 \\
\hline $\mathrm{C}$ & 3.197640 & 0.058409 & -1.264070 \\
\hline $\mathrm{C}$ & 3.196240 & 0.662519 & -0.148750 \\
\hline $\mathrm{C}$ & 3.032560 & 0.728259 & 1.207090 \\
\hline $\mathrm{O}$ & 2.995829 & -2.440501 & -1.973420 \\
\hline B & 2.419409 & -1.503671 & -1.075310 \\
\hline $\mathrm{O}$ & 2.638149 & -1.853061 & 0.343410 \\
\hline $\mathrm{O}$ & 0.999989 & -1.380721 & -1.424440 \\
\hline $\mathrm{C}$ & 2.175260 & -1.088961 & 1.310320 \\
\hline $\mathrm{C}$ & 0.735029 & -2.210540 & -2.591360 \\
\hline $\mathrm{C}$ & 2.496749 & -1.466491 & 2.709610 \\
\hline $\mathrm{C}$ & 3.706349 & -2.100132 & 3.103500 \\
\hline $\mathrm{C}$ & 1.734289 & -1.461351 & 5.020630 \\
\hline $\mathrm{C}$ & 3.890329 & -2.401262 & 4.468320 \\
\hline $\mathrm{C}$ & 2.919059 & -2.088211 & 5.414150 \\
\hline $\mathrm{C}$ & 1.932049 & -3.234411 & -2.534030 \\
\hline $\mathrm{C}$ & 1.661758 & -4.418071 & -1.590280 \\
\hline $\mathrm{C}$ & 2.390349 & -3.754611 & -3.896990 \\
\hline
\end{tabular}




\begin{tabular}{|c|c|c|c|}
\hline $\mathrm{C}$ & -0.654781 & -2.826120 & -2.433030 \\
\hline $\mathrm{C}$ & 0.774029 & -1.309030 & -3.833550 \\
\hline $\mathrm{H}$ & 3.523060 & 0.338528 & -2.255100 \\
\hline $\mathrm{H}$ & 3.924810 & 0.646298 & 1.826940 \\
\hline $\mathrm{H}$ & 2.247660 & 1.361489 & 1.618070 \\
\hline $\mathrm{H}$ & 1.160600 & -0.708361 & 1.177300 \\
\hline $\mathrm{H}$ & 0.970449 & -1.218351 & 5.753520 \\
\hline $\mathrm{H}$ & 4.813429 & -2.886822 & 4.769130 \\
\hline $\mathrm{H}$ & 3.087549 & -2.334761 & 6.458920 \\
\hline $\mathrm{H}$ & 1.325448 & -4.077631 & -0.607260 \\
\hline $\mathrm{H}$ & 2.593728 & -4.974671 & -1.450600 \\
\hline $\mathrm{H}$ & 0.910638 & -5.102301 & -2.000510 \\
\hline $\mathrm{H}$ & 3.234118 & -4.439041 & -3.760190 \\
\hline $\mathrm{H}$ & 2.719919 & -2.941361 & -4.548170 \\
\hline $\mathrm{H}$ & 1.588018 & -4.305881 & -4.401280 \\
\hline $\mathrm{H}$ & -1.420101 & -2.043750 & -2.468650 \\
\hline $\mathrm{H}$ & -0.756781 & -3.348030 & -1.479230 \\
\hline $\mathrm{H}$ & -0.861991 & -3.533040 & -3.244820 \\
\hline $\mathrm{H}$ & 1.768640 & -0.878151 & -3.981570 \\
\hline $\mathrm{H}$ & 0.059700 & -0.488970 & -3.709280 \\
\hline $\mathrm{H}$ & 0.499939 & -1.862170 & -4.738160 \\
\hline $\mathrm{C}$ & -0.019398 & 4.925120 & 2.207620 \\
\hline $\mathrm{C}$ & 0.116942 & 6.347180 & 2.787000 \\
\hline $\mathrm{C}$ & 0.379831 & 3.861480 & 3.248180 \\
\hline $\mathrm{H}$ & -1.079448 & 4.773630 & 1.986650 \\
\hline $\mathrm{H}$ & -0.208527 & 7.102980 & 2.063390 \\
\hline $\mathrm{H}$ & -0.497578 & 6.453280 & 3.689190 \\
\hline $\mathrm{H}$ & 1.153752 & 6.573849 & 3.061880 \\
\hline $\mathrm{H}$ & 0.214921 & 2.852450 & 2.857040 \\
\hline $\mathrm{H}$ & 1.433971 & 3.953709 & 3.536630 \\
\hline $\mathrm{H}$ & -0.222079 & 3.973650 & 4.158170 \\
\hline $\mathrm{C}$ & 0.319331 & 4.125490 & -2.883940 \\
\hline $\mathrm{C}$ & 0.967381 & 2.898569 & -3.552490 \\
\hline $\mathrm{C}$ & 0.392832 & 5.354260 & -3.811800 \\
\hline $\mathrm{H}$ & -0.738359 & 3.885560 & -2.740190 \\
\hline $\mathrm{H}$ & 0.910181 & 2.025419 & -2.897050 \\
\hline $\mathrm{H}$ & 0.447961 & 2.661060 & -4.488900 \\
\hline $\mathrm{H}$ & 2.021011 & 3.077449 & -3.798880 \\
\hline $\mathrm{H}$ & -0.117998 & 6.219850 & -3.375710 \\
\hline $\mathrm{H}$ & 1.432522 & 5.645759 & -4.003600 \\
\hline $\mathrm{H}$ & -0.076958 & 5.135520 & -4.778410 \\
\hline $\mathrm{C}$ & 4.452822 & 5.108978 & -0.125240 \\
\hline $\mathrm{C}$ & 4.839672 & 6.442148 & -0.795380 \\
\hline $\mathrm{C}$ & 5.275071 & 3.944718 & -0.706200 \\
\hline $\mathrm{H}$ & 4.708812 & 5.191738 & 0.940140 \\
\hline $\mathrm{H}$ & 4.290653 & 7.281068 & -0.353940 \\
\hline $\mathrm{H}$ & 5.912982 & 6.637058 & -0.681580 \\
\hline $\mathrm{H}$ & 4.614722 & 6.424128 & -1.868520 \\
\hline $\mathrm{H}$ & 5.023221 & 2.997168 & -0.218040 \\
\hline $\mathrm{H}$ & 5.096081 & 3.824018 & -1.781160 \\
\hline $\mathrm{H}$ & 6.347931 & 4.125247 & -0.569300 \\
\hline $\mathrm{C}$ & -3.694461 & -2.057079 & 2.845510 \\
\hline $\mathrm{C}$ & -2.288441 & -2.514099 & 3.274570 \\
\hline
\end{tabular}




\begin{tabular}{|c|c|c|c|}
\hline $\mathrm{C}$ & -4.753611 & -2.522208 & 3.865430 \\
\hline $\mathrm{H}$ & -3.685710 & -0.962829 & 2.851920 \\
\hline $\mathrm{H}$ & -1.536461 & -2.164830 & 2.563240 \\
\hline $\mathrm{H}$ & -2.047501 & -2.097619 & 4.260420 \\
\hline $\mathrm{H}$ & -2.217161 & -3.605149 & 3.356780 \\
\hline $\mathrm{H}$ & -5.751761 & -2.147488 & 3.612620 \\
\hline $\mathrm{H}$ & -4.810131 & -3.616918 & 3.903760 \\
\hline $\mathrm{H}$ & -4.502781 & -2.163938 & 4.871390 \\
\hline $\mathrm{C}$ & -5.480561 & -1.204948 & -1.919130 \\
\hline $\mathrm{C}$ & -6.912681 & -1.634488 & -2.293940 \\
\hline $\mathrm{C}$ & -4.577801 & -1.134978 & -3.164930 \\
\hline $\mathrm{H}$ & -5.549620 & -0.189968 & -1.517880 \\
\hline $\mathrm{H}$ & -7.566341 & -1.643307 & -1.414480 \\
\hline $\mathrm{H}$ & -7.340940 & -0.942687 & -3.029440 \\
\hline $\mathrm{H}$ & -6.931011 & -2.639358 & -2.732090 \\
\hline $\mathrm{H}$ & -3.580540 & -0.767759 & -2.902550 \\
\hline $\mathrm{H}$ & -4.466391 & -2.117138 & -3.639690 \\
\hline $\mathrm{H}$ & -5.006210 & -0.453368 & -3.910020 \\
\hline $\mathrm{C}$ & -4.087052 & -5.852719 & -0.470010 \\
\hline $\mathrm{C}$ & -3.128162 & -6.118519 & -1.644850 \\
\hline $\mathrm{C}$ & -5.457333 & -6.512888 & -0.717710 \\
\hline $\mathrm{H}$ & -3.652042 & -6.324789 & 0.421610 \\
\hline $\mathrm{H}$ & -2.137592 & -5.692839 & -1.451530 \\
\hline $\mathrm{H}$ & -3.010143 & -7.196119 & -1.811060 \\
\hline $\mathrm{H}$ & -3.505242 & -5.678439 & -2.575750 \\
\hline $\mathrm{H}$ & -6.130202 & -6.357828 & 0.132750 \\
\hline $\mathrm{H}$ & -5.943272 & -6.095358 & -1.607620 \\
\hline $\mathrm{H}$ & -5.345713 & -7.592788 & -0.873760 \\
\hline $\mathrm{C}$ & 4.742359 & -2.423592 & 2.183750 \\
\hline $\mathrm{C}$ & 5.654809 & -2.721742 & 1.436710 \\
\hline $\mathrm{C}$ & 1.532229 & -1.151981 & 3.677590 \\
\hline $\mathrm{H}$ & 0.610540 & -0.672910 & 3.357110 \\
\hline $\mathrm{C}$ & 6.663789 & -3.071413 & 0.493460 \\
\hline $\mathrm{C}$ & 7.986279 & -3.321613 & 0.910630 \\
\hline $\mathrm{C}$ & 6.342109 & -3.173663 & -0.876680 \\
\hline $\mathrm{C}$ & 8.964029 & -3.662894 & -0.019710 \\
\hline $\mathrm{H}$ & 8.232639 & -3.243723 & 1.965410 \\
\hline $\mathrm{C}$ & 7.329719 & -3.517013 & -1.796530 \\
\hline $\mathrm{H}$ & 5.321779 & -2.989452 & -1.201770 \\
\hline $\mathrm{C}$ & 8.639659 & -3.761483 & -1.375220 \\
\hline $\mathrm{H}$ & 9.981068 & -3.852944 & 0.312950 \\
\hline $\mathrm{H}$ & 7.074269 & -3.594533 & -2.850100 \\
\hline $\mathrm{H}$ & 9.404648 & -4.028714 & -2.099510 \\
\hline
\end{tabular}

\section{1d propargylboration TS 2}

B3LYP Energy =

B3LYP Free Energy =

$-3454.928446$

$-3453.726259$

$-3453.244635$

$1(-341.11)$

M06-2X Derived Solution Free Energy (toluene) = Number of Imaginary Frequencies $=$$$
1(-341.11)
$$

B3LYP/6-31G(d) Geometry

C

4.006840

$-1.635108$

$-0.107270$ 


\begin{tabular}{|c|c|c|c|}
\hline $\mathrm{C}$ & 4.455850 & -0.639838 & 0.790290 \\
\hline $\mathrm{C}$ & 5.382109 & 0.307272 & 0.335150 \\
\hline $\mathrm{C}$ & 5.870889 & 0.312572 & -0.970720 \\
\hline $\mathrm{C}$ & 5.398530 & -0.671838 & -1.843490 \\
\hline $\mathrm{C}$ & 4.481520 & -1.647718 & -1.442150 \\
\hline $\mathrm{O}$ & 1.235500 & -1.816360 & -0.850970 \\
\hline $\mathrm{P}$ & 0.118000 & -0.771340 & -0.295860 \\
\hline $\mathrm{C}$ & 1.742211 & -2.803189 & -0.007110 \\
\hline $\mathrm{O}$ & -0.355370 & -0.105230 & -1.638290 \\
\hline $\mathrm{O}$ & -1.074870 & -1.749761 & 0.213230 \\
\hline $\mathrm{C}$ & 0.915341 & -3.888420 & 0.331190 \\
\hline $\mathrm{C}$ & 3.093261 & -2.728929 & 0.366910 \\
\hline $\mathrm{C}$ & -1.388519 & -3.000581 & -0.308670 \\
\hline $\mathrm{C}$ & -0.454859 & -4.050360 & -0.221590 \\
\hline $\mathrm{C}$ & 3.595681 & -3.764659 & 1.167140 \\
\hline $\mathrm{C}$ & 1.462112 & -4.889290 & 1.153040 \\
\hline $\mathrm{C}$ & 2.785902 & -4.823599 & 1.575840 \\
\hline $\mathrm{C}$ & -2.700439 & -3.197462 & -0.772600 \\
\hline $\mathrm{C}$ & -0.860178 & -5.320671 & -0.665710 \\
\hline $\mathrm{C}$ & -3.058858 & -4.489222 & -1.182400 \\
\hline $\mathrm{C}$ & -2.147388 & -5.542041 & -1.141090 \\
\hline $\mathrm{C}$ & -3.724309 & -2.099172 & -0.812550 \\
\hline $\mathrm{C}$ & -4.103060 & -1.530632 & -2.052710 \\
\hline $\mathrm{C}$ & -5.122700 & -0.572843 & -2.074200 \\
\hline $\mathrm{C}$ & -5.776800 & -0.152043 & -0.912900 \\
\hline $\mathrm{C}$ & -5.378220 & -0.721703 & 0.297020 \\
\hline $\mathrm{C}$ & -4.371060 & -1.691022 & 0.375830 \\
\hline $\mathrm{O}$ & 0.520779 & 0.130750 & 0.809130 \\
\hline $\mathrm{H}$ & 5.734949 & 1.069562 & 1.025860 \\
\hline $\mathrm{H}$ & 5.764610 & -0.691798 & -2.867200 \\
\hline $\mathrm{H}$ & 4.639811 & -3.731448 & 1.463310 \\
\hline $\mathrm{H}$ & 0.833552 & -5.721230 & 1.456200 \\
\hline $\mathrm{H}$ & 3.192592 & -5.606109 & 2.210190 \\
\hline $\mathrm{H}$ & -0.142108 & -6.134600 & -0.643550 \\
\hline $\mathrm{H}$ & -4.072238 & -4.658012 & -1.534490 \\
\hline $\mathrm{H}$ & -2.440467 & -6.532291 & -1.477880 \\
\hline $\mathrm{H}$ & -5.417980 & -0.148343 & -3.030600 \\
\hline $\mathrm{H}$ & -5.882860 & -0.411583 & 1.209650 \\
\hline $\mathrm{H}$ & -0.149921 & 0.902410 & -1.679810 \\
\hline $\mathrm{C}$ & -2.346192 & 2.700019 & -1.249600 \\
\hline $\mathrm{C}$ & -2.625112 & 2.490259 & -0.028080 \\
\hline $\mathrm{C}$ & -2.446882 & 2.603509 & 1.318970 \\
\hline $\mathrm{O}$ & -0.778283 & 4.432639 & -2.413570 \\
\hline B & -0.777632 & 3.432509 & -1.408410 \\
\hline $\mathrm{O}$ & -0.497072 & 4.016910 & -0.069000 \\
\hline $\mathrm{O}$ & 0.184008 & 2.403270 & -1.823870 \\
\hline $\mathrm{C}$ & -0.598202 & 3.418699 & 1.097870 \\
\hline $\mathrm{C}$ & 0.765058 & 2.796080 & -3.103710 \\
\hline $\mathrm{C}$ & -0.553922 & 4.347639 & 2.261660 \\
\hline $\mathrm{C}$ & -0.673273 & 5.725829 & 2.030590 \\
\hline $\mathrm{C}$ & -0.387842 & 3.879580 & 3.591530 \\
\hline $\mathrm{C}$ & -0.341163 & 4.821970 & 4.640830 \\
\hline $\mathrm{C}$ & -0.626074 & 6.638909 & 3.078960 \\
\hline
\end{tabular}




\begin{tabular}{|c|c|c|c|}
\hline $\mathrm{C}$ & -0.458513 & 6.183550 & 4.390170 \\
\hline $\mathrm{C}$ & 0.462568 & 4.342100 & -3.133240 \\
\hline $\mathrm{C}$ & 1.521567 & 5.173230 & -2.386850 \\
\hline $\mathrm{C}$ & 0.249227 & 4.924840 & -4.531340 \\
\hline $\mathrm{C}$ & 2.245828 & 2.417981 & -3.085030 \\
\hline $\mathrm{C}$ & 0.040139 & 2.028720 & -4.218480 \\
\hline $\mathrm{H}$ & -2.874852 & 2.448578 & -2.158420 \\
\hline $\mathrm{H}$ & -3.030612 & 3.354908 & 1.849240 \\
\hline $\mathrm{H}$ & -2.190261 & 1.724079 & 1.905950 \\
\hline $\mathrm{H}$ & -0.111662 & 2.445500 & 1.204290 \\
\hline $\mathrm{H}$ & -0.790663 & 6.064689 & 1.007530 \\
\hline $\mathrm{H}$ & -0.209633 & 4.458120 & 5.654850 \\
\hline $\mathrm{H}$ & -0.715224 & 7.702119 & 2.874790 \\
\hline $\mathrm{H}$ & -0.417464 & 6.889010 & 5.215530 \\
\hline $\mathrm{H}$ & 1.706737 & 4.779381 & -1.383790 \\
\hline $\mathrm{H}$ & 1.149237 & 6.197240 & -2.281960 \\
\hline $\mathrm{H}$ & 2.472067 & 5.206601 & -2.930990 \\
\hline $\mathrm{H}$ & 0.036677 & 5.995780 & -4.449170 \\
\hline $\mathrm{H}$ & -0.596783 & 4.454419 & -5.037670 \\
\hline $\mathrm{H}$ & 1.143987 & 4.804040 & -5.153180 \\
\hline $\mathrm{H}$ & 2.356409 & 1.329351 & -3.039470 \\
\hline $\mathrm{H}$ & 2.755258 & 2.840631 & -2.216490 \\
\hline $\mathrm{H}$ & 2.748508 & 2.768861 & -3.993990 \\
\hline $\mathrm{H}$ & -1.022302 & 2.288259 & -4.252260 \\
\hline $\mathrm{H}$ & 0.125989 & 0.952260 & -4.043000 \\
\hline $\mathrm{H}$ & 0.477949 & 2.245140 & -5.198670 \\
\hline $\mathrm{C}$ & -4.048579 & -2.304032 & 1.737910 \\
\hline $\mathrm{C}$ & -5.241719 & -3.117933 & 2.277770 \\
\hline $\mathrm{C}$ & -3.589720 & -1.248152 & 2.759520 \\
\hline $\mathrm{H}$ & -3.218769 & -3.004502 & 1.608740 \\
\hline $\mathrm{H}$ & -5.534809 & -3.905413 & 1.574240 \\
\hline $\mathrm{H}$ & -4.982069 & -3.592603 & 3.231780 \\
\hline $\mathrm{H}$ & -6.117899 & -2.481313 & 2.449100 \\
\hline $\mathrm{H}$ & -2.711550 & -0.708862 & 2.391810 \\
\hline $\mathrm{H}$ & -4.379470 & -0.517472 & 2.971640 \\
\hline $\mathrm{H}$ & -3.316250 & -1.725962 & 3.707360 \\
\hline $\mathrm{C}$ & -3.450960 & -1.938982 & -3.374280 \\
\hline $\mathrm{C}$ & -2.882000 & -0.731342 & -4.141260 \\
\hline $\mathrm{C}$ & -4.425539 & -2.740652 & -4.259600 \\
\hline $\mathrm{H}$ & -2.604519 & -2.593191 & -3.145500 \\
\hline $\mathrm{H}$ & -2.169580 & -0.182521 & -3.519720 \\
\hline $\mathrm{H}$ & -2.359430 & -1.069341 & -5.044370 \\
\hline $\mathrm{H}$ & -3.671260 & -0.039682 & -4.459900 \\
\hline $\mathrm{H}$ & -4.793769 & -3.634992 & -3.744680 \\
\hline $\mathrm{H}$ & -5.297459 & -2.137343 & -4.539700 \\
\hline $\mathrm{H}$ & -3.930249 & -3.061582 & -5.184070 \\
\hline $\mathrm{C}$ & -6.906851 & 0.869007 & -0.956550 \\
\hline $\mathrm{C}$ & -8.143261 & 0.311486 & -1.688350 \\
\hline $\mathrm{C}$ & -6.464171 & 2.210247 & -1.569220 \\
\hline $\mathrm{H}$ & -7.202631 & 1.065136 & 0.083350 \\
\hline $\mathrm{H}$ & -8.486630 & -0.622164 & -1.229600 \\
\hline $\mathrm{H}$ & -8.969541 & 1.032076 & -1.658610 \\
\hline $\mathrm{H}$ & -7.919781 & 0.102976 & -2.741520 \\
\hline
\end{tabular}




\begin{tabular}{|c|c|c|c|}
\hline $\mathrm{H}$ & -5.607382 & 2.630217 & -1.031300 \\
\hline $\mathrm{H}$ & -6.175021 & 2.092987 & -2.620550 \\
\hline $\mathrm{H}$ & -7.281502 & 2.940426 & -1.531810 \\
\hline $\mathrm{C}$ & 3.979310 & -0.558508 & 2.240080 \\
\hline $\mathrm{C}$ & 3.407439 & 0.828841 & 2.586350 \\
\hline $\mathrm{C}$ & 5.102070 & -0.948868 & 3.222100 \\
\hline $\mathrm{H}$ & 3.163900 & -1.276219 & 2.370900 \\
\hline $\mathrm{H}$ & 2.591809 & 1.089611 & 1.907270 \\
\hline $\mathrm{H}$ & 3.010129 & 0.825551 & 3.607820 \\
\hline $\mathrm{H}$ & 4.173929 & 1.611462 & 2.533140 \\
\hline $\mathrm{H}$ & 5.480350 & -1.958568 & 3.024740 \\
\hline $\mathrm{H}$ & 5.950920 & -0.258057 & 3.149220 \\
\hline $\mathrm{H}$ & 4.734540 & -0.920548 & 4.254890 \\
\hline $\mathrm{C}$ & 4.059351 & -2.709158 & -2.458770 \\
\hline $\mathrm{C}$ & 5.257471 & -3.569318 & -2.907510 \\
\hline $\mathrm{C}$ & 3.337631 & -2.089189 & -3.670550 \\
\hline $\mathrm{H}$ & 3.349161 & -3.385159 & -1.974890 \\
\hline $\mathrm{H}$ & 5.746321 & -4.046358 & -2.050640 \\
\hline $\mathrm{H}$ & 4.926972 & -4.358458 & -3.593850 \\
\hline $\mathrm{H}$ & 6.012021 & -2.969187 & -3.429420 \\
\hline $\mathrm{H}$ & 2.456130 & -1.524169 & -3.351830 \\
\hline $\mathrm{H}$ & 3.995990 & -1.412468 & -4.228520 \\
\hline $\mathrm{H}$ & 3.008301 & -2.874809 & -4.361700 \\
\hline $\mathrm{C}$ & 6.895749 & 1.348503 & -1.414520 \\
\hline $\mathrm{C}$ & 6.379679 & 2.219723 & -2.574720 \\
\hline $\mathrm{C}$ & 8.244369 & 0.695984 & -1.774370 \\
\hline $\mathrm{H}$ & 7.071559 & 2.014013 & -0.558150 \\
\hline $\mathrm{H}$ & 5.437628 & 2.712402 & -2.311200 \\
\hline $\mathrm{H}$ & 7.111948 & 2.995073 & -2.830690 \\
\hline $\mathrm{H}$ & 6.202569 & 1.620383 & -3.475740 \\
\hline $\mathrm{H}$ & 8.635979 & 0.108554 & -0.936690 \\
\hline $\mathrm{H}$ & 8.142810 & 0.023494 & -2.634590 \\
\hline $\mathrm{H}$ & 8.987899 & 1.459434 & -2.033860 \\
\hline $\mathrm{C}$ & -0.277602 & 2.500560 & 3.927250 \\
\hline $\mathrm{C}$ & -0.176911 & 1.354640 & 4.326160 \\
\hline $\mathrm{C}$ & -0.051960 & 0.020490 & 4.812830 \\
\hline $\mathrm{C}$ & -0.154680 & -0.239050 & 6.194870 \\
\hline $\mathrm{C}$ & 0.181000 & -1.049480 & 3.925090 \\
\hline $\mathrm{C}$ & -0.027190 & -1.539050 & 6.675320 \\
\hline $\mathrm{H}$ & -0.333801 & 0.585830 & 6.878060 \\
\hline $\mathrm{C}$ & 0.308481 & -2.345850 & 4.419230 \\
\hline $\mathrm{H}$ & 0.263310 & -0.843740 & 2.862710 \\
\hline $\mathrm{C}$ & 0.205031 & -2.595540 & 5.790260 \\
\hline $\mathrm{H}$ & -0.107870 & -1.728730 & 7.742370 \\
\hline $\mathrm{H}$ & 0.493321 & -3.163440 & 3.727610 \\
\hline $\mathrm{H}$ & 0.306021 & -3.609240 & 6.169050 \\
\hline
\end{tabular}

1d propargylboration TS 3

B3LYP Energy $=$

$-3454.928001$

$-3453.725661$

$-3453.239452$

M06-2X Derived Solution Free Energy (toluene) =

$1(-317.53)$ 
B3LYP/6-31G(d) Geometry

\begin{tabular}{|c|c|c|c|}
\hline B & 2.010370 & -1.266720 & -0.711179 \\
\hline $\mathrm{C}$ & 1.757650 & -0.834370 & 0.939041 \\
\hline $\mathrm{C}$ & 2.857350 & -0.456060 & 1.453421 \\
\hline $\mathrm{C}$ & 4.148970 & -0.031640 & 1.451231 \\
\hline $\mathrm{H}$ & 0.754070 & -0.927910 & 1.344881 \\
\hline $\mathrm{H}$ & 4.939160 & -0.653880 & 1.867231 \\
\hline $\mathrm{H}$ & 4.342150 & 1.040160 & 1.471371 \\
\hline $\mathrm{O}$ & 2.334380 & -2.653380 & -0.815939 \\
\hline $\mathrm{C}$ & 1.716240 & -3.153040 & -2.017519 \\
\hline $\mathrm{O}$ & 0.844720 & -1.018650 & -1.538739 \\
\hline $\mathrm{C}$ & 0.403020 & -2.287440 & -2.108979 \\
\hline $\mathrm{C}$ & 2.671750 & -2.915090 & -3.200819 \\
\hline $\mathrm{H}$ & 2.846470 & -1.848590 & -3.368069 \\
\hline $\mathrm{H}$ & 3.634170 & -3.385650 & -2.973809 \\
\hline $\mathrm{H}$ & 3.912690 & 3.501560 & 1.990931 \\
\hline $\mathrm{C}$ & 1.483461 & -4.654180 & -1.841169 \\
\hline $\mathrm{H}$ & 2.449141 & -5.167150 & -1.779319 \\
\hline $\mathrm{H}$ & 0.930811 & -4.866020 & -0.923189 \\
\hline $\mathrm{H}$ & 0.931921 & -5.074000 & -2.690509 \\
\hline $\mathrm{C}$ & -0.102500 & -2.015330 & -3.525789 \\
\hline $\mathrm{H}$ & 0.649590 & -1.505640 & -4.132209 \\
\hline $\mathrm{H}$ & -0.992360 & -1.378140 & -3.485339 \\
\hline $\mathrm{H}$ & -0.382660 & -2.950160 & -4.025119 \\
\hline $\mathrm{C}$ & -0.733300 & -2.841740 & -1.240759 \\
\hline $\mathrm{H}$ & -0.403490 & -3.017160 & -0.213349 \\
\hline $\mathrm{H}$ & -1.564690 & -2.132290 & -1.210799 \\
\hline $\mathrm{H}$ & -1.121889 & -3.780450 & -1.648089 \\
\hline $\mathrm{O}$ & 3.128390 & -0.348730 & -1.153089 \\
\hline $\mathrm{C}$ & 4.297200 & -0.521750 & -0.600919 \\
\hline $\mathrm{H}$ & 4.574180 & -1.549270 & -0.363929 \\
\hline $\mathrm{C}$ & 5.388610 & 0.383110 & -1.018019 \\
\hline $\mathrm{C}$ & 6.739790 & 0.051501 & -0.731159 \\
\hline $\mathrm{C}$ & 7.759910 & 0.917551 & -1.170979 \\
\hline $\mathrm{C}$ & 6.122090 & 2.394341 & -2.161779 \\
\hline $\mathrm{C}$ & 7.454110 & 2.076391 & -1.876469 \\
\hline $\mathrm{H}$ & 8.791800 & 0.661171 & -0.953119 \\
\hline $\mathrm{H}$ & 5.883360 & 3.294071 & -2.721539 \\
\hline $\mathrm{H}$ & 8.255070 & 2.730311 & -2.209679 \\
\hline $\mathrm{H}$ & -0.268930 & 0.057840 & -1.198649 \\
\hline $\mathrm{O}$ & -1.003290 & 0.752650 & -1.070889 \\
\hline $\mathrm{P}$ & -1.875180 & 0.591970 & 0.232451 \\
\hline $\mathrm{O}$ & -1.363790 & -0.283880 & 1.309511 \\
\hline $\mathrm{O}$ & -3.314580 & 0.179080 & -0.414759 \\
\hline $\mathrm{O}$ & -2.144960 & 2.091640 & 0.791031 \\
\hline $\mathrm{C}$ & -4.521220 & 0.443970 & 0.228291 \\
\hline $\mathrm{C}$ & -4.969070 & 1.774820 & 0.304221 \\
\hline $\mathrm{C}$ & -5.304230 & -0.639850 & 0.656641 \\
\hline $\mathrm{C}$ & -6.232950 & 2.008830 & 0.870361 \\
\hline $\mathrm{C}$ & -6.565250 & -0.353841 & 1.199311 \\
\hline $\mathrm{C}$ & -7.025400 & 0.956439 & 1.315241 \\
\hline $\mathrm{H}$ & -6.581080 & 3.032569 & 0.968891 \\
\hline $\mathrm{H}$ & -7.187470 & -1.181531 & 1.527111 \\
\hline
\end{tabular}




\begin{tabular}{|c|c|c|c|}
\hline $\mathrm{H}$ & -8.000320 & 1.155839 & 1.750961 \\
\hline $\mathrm{C}$ & -2.802900 & 3.065270 & 0.042831 \\
\hline $\mathrm{C}$ & -4.178130 & 2.920920 & -0.211429 \\
\hline $\mathrm{C}$ & -2.086760 & 4.221030 & -0.305959 \\
\hline $\mathrm{C}$ & -4.822370 & 3.950350 & -0.917209 \\
\hline $\mathrm{C}$ & -2.781710 & 5.228980 & -0.989349 \\
\hline $\mathrm{C}$ & -4.130800 & 5.090200 & -1.311749 \\
\hline $\mathrm{H}$ & -5.875890 & 3.841080 & -1.156229 \\
\hline $\mathrm{H}$ & -2.249150 & 6.137350 & -1.254209 \\
\hline $\mathrm{H}$ & -4.644890 & 5.877330 & -1.856019 \\
\hline $\mathrm{C}$ & -4.867420 & -2.069920 & 0.516841 \\
\hline $\mathrm{C}$ & -4.383580 & -2.776590 & 1.641011 \\
\hline $\mathrm{C}$ & -5.023660 & -2.733080 & -0.723619 \\
\hline $\mathrm{C}$ & -4.080729 & -4.136620 & 1.498911 \\
\hline $\mathrm{C}$ & -4.698999 & -4.090470 & -0.810039 \\
\hline $\mathrm{C}$ & -4.232259 & -4.816170 & 0.289991 \\
\hline $\mathrm{H}$ & -3.717789 & -4.687910 & 2.363341 \\
\hline $\mathrm{H}$ & -4.829619 & -4.598580 & -1.762689 \\
\hline $\mathrm{C}$ & -0.658170 & 4.448350 & 0.099581 \\
\hline $\mathrm{C}$ & -0.367120 & 4.827070 & 1.430551 \\
\hline $\mathrm{C}$ & 0.382100 & 4.380230 & -0.858229 \\
\hline $\mathrm{C}$ & 0.951930 & 5.152800 & 1.765381 \\
\hline $\mathrm{C}$ & 1.681940 & 4.722080 & -0.468449 \\
\hline $\mathrm{C}$ & 1.990520 & 5.120440 & 0.835151 \\
\hline $\mathrm{H}$ & 1.176670 & 5.453870 & 2.786541 \\
\hline $\mathrm{H}$ & 2.475560 & 4.679590 & -1.209859 \\
\hline $\mathrm{C}$ & -4.216310 & -2.121190 & 3.011931 \\
\hline $\mathrm{C}$ & -2.811650 & -2.334260 & 3.606291 \\
\hline $\mathrm{C}$ & -5.305010 & -2.604740 & 3.991471 \\
\hline $\mathrm{H}$ & -4.344910 & -1.041810 & 2.885631 \\
\hline $\mathrm{H}$ & -2.044560 & -1.942640 & 2.933891 \\
\hline $\mathrm{H}$ & -2.729720 & -1.804610 & 4.563401 \\
\hline $\mathrm{H}$ & -2.605930 & -3.393750 & 3.801431 \\
\hline $\mathrm{H}$ & -6.311840 & -2.402451 & 3.608941 \\
\hline $\mathrm{H}$ & -5.227689 & -3.684700 & 4.167211 \\
\hline $\mathrm{H}$ & -5.202530 & -2.099660 & 4.959671 \\
\hline $\mathrm{C}$ & -3.917109 & -6.303480 & 0.188731 \\
\hline $\mathrm{C}$ & -2.776869 & -6.590220 & -0.805769 \\
\hline $\mathrm{C}$ & -5.170299 & -7.129610 & -0.159119 \\
\hline $\mathrm{H}$ & -3.575069 & -6.627800 & 1.181121 \\
\hline $\mathrm{H}$ & -1.868569 & -6.041460 & -0.534279 \\
\hline $\mathrm{H}$ & -2.537229 & -7.660440 & -0.822859 \\
\hline $\mathrm{H}$ & -3.054299 & -6.295870 & -1.825149 \\
\hline $\mathrm{H}$ & -5.971479 & -6.958520 & 0.568141 \\
\hline $\mathrm{H}$ & -5.557749 & -6.865090 & -1.150289 \\
\hline $\mathrm{H}$ & -4.938419 & -8.201600 & -0.167229 \\
\hline $\mathrm{C}$ & -5.573890 & -2.027340 & -1.963199 \\
\hline $\mathrm{C}$ & -6.921940 & -2.631111 & -2.403979 \\
\hline $\mathrm{C}$ & -4.562120 & -2.023090 & -3.124199 \\
\hline $\mathrm{H}$ & -5.764550 & -0.982320 & -1.702689 \\
\hline $\mathrm{H}$ & -7.657110 & -2.592541 & -1.592289 \\
\hline $\mathrm{H}$ & -7.329730 & -2.077611 & -3.258609 \\
\hline $\mathrm{H}$ & -6.815159 & -3.679581 & -2.706519 \\
\hline
\end{tabular}




\begin{tabular}{|c|c|c|c|}
\hline $\mathrm{H}$ & -3.627660 & -1.538700 & -2.824089 \\
\hline $\mathrm{H}$ & -4.326290 & -3.040070 & -3.459809 \\
\hline $\mathrm{H}$ & -4.968310 & -1.475750 & -3.983589 \\
\hline $\mathrm{C}$ & 0.126350 & 3.971200 & -2.309659 \\
\hline $\mathrm{C}$ & 1.108960 & 2.896140 & -2.810999 \\
\hline $\mathrm{C}$ & 0.155100 & 5.194980 & -3.248259 \\
\hline $\mathrm{H}$ & -0.874710 & 3.532840 & -2.362269 \\
\hline $\mathrm{H}$ & 1.122170 & 2.022780 & -2.153739 \\
\hline $\mathrm{H}$ & 0.814840 & 2.560440 & -3.812579 \\
\hline $\mathrm{H}$ & 2.132210 & 3.284960 & -2.889079 \\
\hline $\mathrm{H}$ & -0.590730 & 5.943930 & -2.961529 \\
\hline $\mathrm{H}$ & 1.138130 & 5.681470 & -3.228439 \\
\hline $\mathrm{H}$ & -0.050130 & 4.893090 & -4.282669 \\
\hline $\mathrm{C}$ & -1.437550 & 4.921860 & 2.517461 \\
\hline $\mathrm{C}$ & -1.589070 & 6.363550 & 3.041151 \\
\hline $\mathrm{C}$ & -1.166320 & 3.935480 & 3.669571 \\
\hline $\mathrm{H}$ & -2.398640 & 4.642980 & 2.077351 \\
\hline $\mathrm{H}$ & -1.826330 & 7.058570 & 2.227731 \\
\hline $\mathrm{H}$ & -2.396230 & 6.418350 & 3.781671 \\
\hline $\mathrm{H}$ & -0.670560 & 6.716940 & 3.524451 \\
\hline $\mathrm{H}$ & -1.105080 & 2.908230 & 3.297961 \\
\hline $\mathrm{H}$ & -0.227650 & 4.167990 & 4.186941 \\
\hline $\mathrm{H}$ & -1.973750 & 3.984180 & 4.410451 \\
\hline $\mathrm{C}$ & 3.407600 & 5.494980 & 1.250961 \\
\hline $\mathrm{C}$ & 4.016610 & 6.588730 & 0.355221 \\
\hline $\mathrm{C}$ & 4.325770 & 4.259180 & 1.315111 \\
\hline $\mathrm{H}$ & 3.343720 & 5.905060 & 2.268331 \\
\hline $\mathrm{H}$ & 3.382090 & 7.481460 & 0.333131 \\
\hline $\mathrm{H}$ & 5.006820 & 6.881930 & 0.724271 \\
\hline $\mathrm{H}$ & 4.139150 & 6.241170 & -0.677419 \\
\hline $\mathrm{H}$ & 2.288070 & -3.354450 & -4.128229 \\
\hline $\mathrm{H}$ & 4.444030 & 3.800010 & 0.326541 \\
\hline $\mathrm{H}$ & 5.324000 & 4.533350 & 1.678221 \\
\hline $\mathrm{C}$ & 7.079610 & -1.133299 & -0.018059 \\
\hline $\mathrm{C}$ & 7.373840 & -2.149199 & 0.584981 \\
\hline $\mathrm{C}$ & 5.099720 & 1.551590 & -1.734229 \\
\hline $\mathrm{H}$ & 4.062270 & 1.776790 & -1.958239 \\
\hline $\mathrm{C}$ & 7.707090 & -3.341289 & 1.290991 \\
\hline $\mathrm{C}$ & 9.043611 & -3.614449 & 1.645111 \\
\hline $\mathrm{C}$ & 6.702201 & -4.264359 & 1.645731 \\
\hline $\mathrm{C}$ & 9.361881 & -4.779539 & 2.336831 \\
\hline $\mathrm{H}$ & 9.819380 & -2.905639 & 1.372361 \\
\hline $\mathrm{C}$ & 7.031191 & -5.426239 & 2.337891 \\
\hline $\mathrm{H}$ & 5.671611 & -4.060820 & 1.370881 \\
\hline $\mathrm{C}$ & 8.358981 & -5.687819 & 2.685651 \\
\hline $\mathrm{H}$ & 10.395541 & -4.979959 & 2.605021 \\
\hline $\mathrm{H}$ & 6.248671 & -6.130439 & 2.606381 \\
\hline $\mathrm{H}$ & 8.611231 & -6.596089 & 3.225851 \\
\hline
\end{tabular}

\section{1d propargylboration TS 4}

B3LYP Energy =

$-3454.920526$

B3LYP Free Energy =

$-3453.717902$

M06-2X Derived Solution Free Energy (toluene) $=$

$-3453.235019$ 
B3LYP/6-31G(d) Geometry

\begin{tabular}{|c|c|c|c|}
\hline B & -0.504505 & 3.371224 & -0.560423 \\
\hline $\mathrm{C}$ & 0.013844 & 3.281912 & 1.092406 \\
\hline $\mathrm{C}$ & -0.753129 & 4.017472 & 1.792517 \\
\hline $\mathrm{C}$ & -1.881073 & 4.728920 & 2.052357 \\
\hline $\mathrm{H}$ & 0.900251 & 2.711785 & 1.337297 \\
\hline $\mathrm{H}$ & -1.834148 & 5.813922 & 2.137637 \\
\hline $\mathrm{H}$ & -2.675283 & 4.244803 & 2.617475 \\
\hline $\mathrm{O}$ & 0.027007 & 4.564506 & -1.146074 \\
\hline $\mathrm{C}$ & 0.466694 & 4.251098 & -2.479981 \\
\hline $\mathrm{O}$ & -0.066607 & 2.253124 & -1.370949 \\
\hline $\mathrm{C}$ & 0.907728 & 2.750114 & -2.338541 \\
\hline $\mathrm{C}$ & -0.717435 & 4.434974 & -3.445771 \\
\hline $\mathrm{H}$ & -1.534140 & 3.745374 & -3.212979 \\
\hline $\mathrm{H}$ & -1.095511 & 5.458200 & -3.347671 \\
\hline $\mathrm{H}$ & -5.814203 & -0.144709 & 0.527974 \\
\hline $\mathrm{C}$ & 1.592682 & 5.216557 & -2.852471 \\
\hline $\mathrm{H}$ & 1.196835 & 6.236333 & -2.905904 \\
\hline $\mathrm{H}$ & 2.391185 & 5.205692 & -2.107292 \\
\hline $\mathrm{H}$ & 2.020313 & 4.968970 & -3.831074 \\
\hline $\mathrm{C}$ & 0.795479 & 1.919978 & -3.616606 \\
\hline $\mathrm{H}$ & -0.230382 & 1.895501 & -3.989985 \\
\hline $\mathrm{H}$ & 1.110566 & 0.889331 & -3.426142 \\
\hline $\mathrm{H}$ & 1.443747 & 2.332839 & -4.398301 \\
\hline $\mathrm{C}$ & 2.306272 & 2.595441 & -1.726931 \\
\hline $\mathrm{H}$ & 2.421538 & 3.216271 & -0.833959 \\
\hline $\mathrm{H}$ & 2.470910 & 1.552311 & -1.441118 \\
\hline $\mathrm{H}$ & 3.087894 & 2.873506 & -2.441372 \\
\hline $\mathrm{O}$ & -2.003627 & 3.371687 & -0.426905 \\
\hline $\mathrm{C}$ & -2.538968 & 4.463222 & 0.043508 \\
\hline $\mathrm{H}$ & -2.054766 & 5.391262 & -0.267732 \\
\hline $\mathrm{C}$ & -4.013230 & 4.530948 & 0.180940 \\
\hline $\mathrm{C}$ & -4.637624 & 5.652310 & -0.381538 \\
\hline $\mathrm{C}$ & -4.808976 & 3.527853 & 0.795758 \\
\hline $\mathrm{C}$ & -6.024734 & 5.781474 & -0.392908 \\
\hline $\mathrm{C}$ & -6.210459 & 3.674314 & 0.772345 \\
\hline $\mathrm{C}$ & -6.811637 & 4.780139 & 0.179413 \\
\hline $\mathrm{H}$ & -4.021620 & 6.422882 & -0.838616 \\
\hline $\mathrm{H}$ & -6.485681 & 6.653010 & -0.848206 \\
\hline $\mathrm{H}$ & -6.816306 & 2.906621 & 1.242789 \\
\hline $\mathrm{H}$ & -7.894677 & 4.865712 & 0.175719 \\
\hline $\mathrm{H}$ & -0.003906 & 0.670793 & -1.132352 \\
\hline $\mathrm{O}$ & 0.095999 & -0.338162 & -1.174202 \\
\hline $\mathrm{P}$ & 0.817306 & -1.013762 & 0.053162 \\
\hline $\mathrm{O}$ & 0.824441 & -0.290030 & 1.344075 \\
\hline $\mathrm{O}$ & 2.283741 & -1.345833 & -0.584966 \\
\hline $\mathrm{O}$ & 0.139620 & -2.479301 & 0.200537 \\
\hline $\mathrm{C}$ & 3.092223 & -2.334857 & -0.026129 \\
\hline $\mathrm{C}$ & 2.730025 & -3.680588 & -0.212523 \\
\hline $\mathrm{C}$ & 4.296975 & -1.956115 & 0.587739 \\
\hline $\mathrm{C}$ & 3.592087 & -4.666882 & 0.293458 \\
\hline
\end{tabular}




\begin{tabular}{|c|c|c|c|}
\hline $\mathrm{C}$ & 5.131141 & -2.979282 & 1.060516 \\
\hline $\mathrm{C}$ & 4.779634 & -4.321798 & 0.929413 \\
\hline $\mathrm{H}$ & 3.312284 & -5.710772 & 0.188841 \\
\hline $\mathrm{H}$ & 6.073199 & -2.705960 & 1.526723 \\
\hline $\mathrm{H}$ & 5.434900 & -5.097124 & 1.316170 \\
\hline $\mathrm{C}$ & 0.261105 & -3.477953 & -0.765777 \\
\hline $\mathrm{C}$ & 1.515432 & -4.081429 & -0.967404 \\
\hline $\mathrm{C}$ & -0.906062 & -3.935548 & -1.399263 \\
\hline $\mathrm{C}$ & 1.593545 & -5.138303 & -1.889046 \\
\hline $\mathrm{C}$ & -0.775820 & -5.005555 & -2.296524 \\
\hline C & 0.460766 & -5.594532 & -2.553378 \\
\hline $\mathrm{H}$ & 2.560367 & -5.592823 & -2.083349 \\
\hline $\mathrm{H}$ & -1.670229 & -5.383192 & -2.782743 \\
\hline $\mathrm{H}$ & 0.538235 & -6.413862 & -3.262465 \\
\hline $\mathrm{C}$ & 4.746769 & -0.525797 & 0.682599 \\
\hline $\mathrm{C}$ & 4.563787 & 0.207802 & 1.876730 \\
\hline $\mathrm{C}$ & 5.431739 & 0.056723 & -0.410449 \\
\hline $\mathrm{C}$ & 5.070133 & 1.511670 & 1.944856 \\
\hline $\mathrm{C}$ & 5.914597 & 1.363847 & -0.287670 \\
\hline $\mathrm{C}$ & 5.746325 & 2.111320 & 0.881180 \\
\hline $\mathrm{H}$ & 4.940362 & 2.080827 & 2.862142 \\
\hline $\mathrm{H}$ & 6.449664 & 1.803785 & -1.125941 \\
\hline $\mathrm{C}$ & -2.274068 & -3.392678 & -1.094554 \\
\hline $\mathrm{C}$ & -2.959892 & -3.852979 & 0.051786 \\
\hline C & -2.912408 & -2.500204 & -1.989531 \\
\hline $\mathrm{C}$ & -4.265856 & -3.404880 & 0.282607 \\
\hline $\mathrm{C}$ & -4.223114 & -2.096701 & -1.716583 \\
\hline $\mathrm{C}$ & -4.919945 & -2.532057 & -0.585995 \\
\hline $\mathrm{H}$ & -4.793730 & -3.754584 & 1.167262 \\
\hline $\mathrm{H}$ & -4.714916 & -1.417875 & -2.407641 \\
\hline $\mathrm{C}$ & 3.889678 & -0.399454 & 3.107760 \\
\hline $\mathrm{C}$ & 2.877995 & 0.546162 & 3.778299 \\
\hline $\mathrm{C}$ & 4.946655 & -0.869946 & 4.128629 \\
\hline $\mathrm{H}$ & 3.327620 & -1.279300 & 2.779913 \\
\hline $\mathrm{H}$ & 2.108296 & 0.854547 & 3.067307 \\
\hline $\mathrm{H}$ & 2.381087 & 0.027929 & 4.607622 \\
\hline $\mathrm{H}$ & 3.359168 & 1.437463 & 4.198380 \\
\hline $\mathrm{H}$ & 5.632104 & -1.604442 & 3.692272 \\
\hline $\mathrm{H}$ & 5.547526 & -0.025229 & 4.487470 \\
\hline $\mathrm{H}$ & 4.463786 & -1.333077 & 4.997827 \\
\hline $\mathrm{C}$ & 6.305514 & 3.522222 & 1.015412 \\
\hline $\mathrm{C}$ & 5.739350 & 4.481897 & -0.047206 \\
\hline $\mathrm{C}$ & 7.846355 & 3.524520 & 0.993428 \\
\hline $\mathrm{H}$ & 5.991538 & 3.899403 & 1.998375 \\
\hline $\mathrm{H}$ & 4.644777 & 4.509811 & -0.012783 \\
\hline $\mathrm{H}$ & 6.112163 & 5.500557 & 0.114412 \\
\hline $\mathrm{H}$ & 6.034483 & 4.177725 & -1.058553 \\
\hline $\mathrm{H}$ & 8.253827 & 2.874747 & 1.775660 \\
\hline $\mathrm{H}$ & 8.229522 & 3.167068 & 0.030111 \\
\hline $\mathrm{H}$ & 8.235705 & 4.537464 & 1.152770 \\
\hline $\mathrm{C}$ & 5.710207 & -0.714275 & -1.701675 \\
\hline C & 7.216456 & -1.002732 & -1.860274 \\
\hline $\mathrm{C}$ & 5.149815 & -0.006265 & -2.948557 \\
\hline
\end{tabular}




\begin{tabular}{|c|c|c|c|}
\hline $\mathrm{H}$ & 5.206135 & -1.682485 & -1.634926 \\
\hline $\mathrm{H}$ & 7.605609 & -1.559224 & -1.000275 \\
\hline $\mathrm{H}$ & 7.402096 & -1.597985 & -2.762682 \\
\hline $\mathrm{H}$ & 7.795130 & -0.075278 & -1.946059 \\
\hline $\mathrm{H}$ & 4.070178 & 0.149415 & -2.859269 \\
\hline $\mathrm{H}$ & 5.620598 & 0.970736 & -3.110896 \\
\hline $\mathrm{H}$ & 5.333194 & -0.612961 & -3.843693 \\
\hline $\mathrm{C}$ & -2.230000 & -1.998223 & -3.262410 \\
\hline $\mathrm{C}$ & -2.410471 & -0.485662 & -3.486520 \\
\hline C & -2.720901 & -2.778543 & -4.499529 \\
\hline $\mathrm{H}$ & -1.155652 & -2.179215 & -3.158945 \\
\hline $\mathrm{H}$ & -2.092962 & 0.088873 & -2.612593 \\
\hline $\mathrm{H}$ & -1.807346 & -0.164617 & -4.344380 \\
\hline $\mathrm{H}$ & -3.451810 & -0.224761 & -3.709388 \\
\hline $\mathrm{H}$ & -2.534055 & -3.853057 & -4.402206 \\
\hline $\mathrm{H}$ & -3.799126 & -2.640400 & -4.646515 \\
\hline $\mathrm{H}$ & -2.210060 & -2.428021 & -5.404949 \\
\hline $\mathrm{C}$ & -2.340850 & -4.843392 & 1.037431 \\
\hline $\mathrm{C}$ & -3.129597 & -6.166858 & 1.081090 \\
\hline $\mathrm{C}$ & -2.195637 & -4.234574 & 2.443894 \\
\hline $\mathrm{H}$ & -1.333239 & -5.087100 & 0.688830 \\
\hline $\mathrm{H}$ & -3.198349 & -6.621511 & 0.086269 \\
\hline $\mathrm{H}$ & -2.637481 & -6.883844 & 1.749663 \\
\hline $\mathrm{H}$ & -4.151063 & -6.016238 & 1.449996 \\
\hline $\mathrm{H}$ & -1.599187 & -3.318515 & 2.412334 \\
\hline $\mathrm{H}$ & -3.169924 & -3.983414 & 2.879365 \\
\hline $\mathrm{H}$ & -1.702443 & -4.945876 & 3.118417 \\
\hline $\mathrm{C}$ & -6.353300 & -2.093180 & -0.314875 \\
\hline $\mathrm{C}$ & -7.327346 & -2.673856 & -1.358456 \\
\hline $\mathrm{C}$ & -6.490117 & -0.562086 & -0.225577 \\
\hline $\mathrm{H}$ & -6.636237 & -2.506228 & 0.663243 \\
\hline $\mathrm{H}$ & -7.267198 & -3.767362 & -1.390730 \\
\hline $\mathrm{H}$ & -8.361816 & -2.394491 & -1.123265 \\
\hline $\mathrm{H}$ & -7.099808 & -2.297962 & -2.363237 \\
\hline $\mathrm{H}$ & -0.420504 & 4.282128 & -4.489140 \\
\hline $\mathrm{H}$ & -6.254027 & -0.083338 & -1.183208 \\
\hline $\mathrm{H}$ & -7.518308 & -0.284094 & 0.038373 \\
\hline $\mathrm{C}$ & -4.245402 & 2.443268 & 1.522476 \\
\hline $\mathrm{C}$ & -3.821795 & 1.568889 & 2.256401 \\
\hline $\mathrm{C}$ & -3.296129 & 0.586413 & 3.144569 \\
\hline $\mathrm{C}$ & -1.945425 & 0.196950 & 3.056329 \\
\hline $\mathrm{C}$ & -4.114032 & 0.019978 & 4.143810 \\
\hline $\mathrm{C}$ & -1.427315 & -0.731779 & 3.954846 \\
\hline $\mathrm{H}$ & -1.306676 & 0.613187 & 2.285927 \\
\hline $\mathrm{C}$ & -3.584983 & -0.904984 & 5.038918 \\
\hline $\mathrm{H}$ & -5.155925 & 0.318950 & 4.210614 \\
\hline $\mathrm{C}$ & -2.241046 & -1.280436 & 4.949436 \\
\hline $\mathrm{H}$ & -0.386087 & -1.024028 & 3.860843 \\
\hline $\mathrm{H}$ & -4.220926 & -1.333212 & 5.809232 \\
\hline $\mathrm{H}$ & -1.832137 & -2.003183 & 5.650593 \\
\hline
\end{tabular}

1a allylboration TS 3

B3LYP Energy =

$-3225.109439$ 
B3LYP Free Energy =

$-3223.961741$

M06-2X Derived Solution Free Energy $(\mathrm{DCM})=$

$-3223.522077$

M06-2X Derived Solution Free Energy (toluene) =

B3LYP/6-31G(d) Geometry

\begin{tabular}{|c|c|c|c|}
\hline B & 0.355603 & 3.055200 & -1.243790 \\
\hline $\mathrm{C}$ & 2.057113 & 2.868439 & -0.944320 \\
\hline $\mathrm{C}$ & 2.383164 & 4.060789 & -0.211560 \\
\hline $\mathrm{C}$ & 2.182884 & 4.186849 & 1.145470 \\
\hline $\mathrm{H}$ & 2.493313 & 2.813979 & -1.943960 \\
\hline $\mathrm{H}$ & 2.183292 & 1.936449 & -0.389770 \\
\hline $\mathrm{H}$ & 2.550384 & 4.969529 & -0.790720 \\
\hline $\mathrm{H}$ & 2.354224 & 5.135349 & 1.646850 \\
\hline $\mathrm{H}$ & 2.182983 & 3.302009 & 1.773770 \\
\hline $\mathrm{O}$ & 0.097944 & 4.237071 & -2.009660 \\
\hline $\mathrm{C}$ & -0.954826 & 3.939201 & -2.943240 \\
\hline $\mathrm{O}$ & -0.235708 & 1.934391 & -1.953440 \\
\hline $\mathrm{C}$ & -0.720678 & 2.410851 & -3.237110 \\
\hline $\mathrm{C}$ & -2.308846 & 4.211422 & -2.263800 \\
\hline $\mathrm{H}$ & -2.462157 & 3.551463 & -1.404830 \\
\hline $\mathrm{H}$ & -2.319245 & 5.245883 & -1.904700 \\
\hline $\mathrm{H}$ & -3.148796 & 4.082063 & -2.955170 \\
\hline $\mathrm{C}$ & -0.793726 & 4.853901 & -4.158290 \\
\hline $\mathrm{H}$ & -0.961655 & 5.893911 & -3.858740 \\
\hline $\mathrm{H}$ & 0.211874 & 4.784910 & -4.579560 \\
\hline $\mathrm{H}$ & -1.520746 & 4.606582 & -4.940790 \\
\hline $\mathrm{C}$ & -1.979028 & 1.631542 & -3.614510 \\
\hline $\mathrm{H}$ & -2.712578 & 1.630413 & -2.805010 \\
\hline $\mathrm{H}$ & -1.725049 & 0.591832 & -3.840460 \\
\hline $\mathrm{H}$ & -2.443918 & 2.062473 & -4.509220 \\
\hline $\mathrm{C}$ & 0.379802 & 2.159970 & -4.279300 \\
\hline $\mathrm{H}$ & 1.274513 & 2.751890 & -4.065980 \\
\hline $\mathrm{H}$ & 0.656701 & 1.100860 & -4.256740 \\
\hline $\mathrm{H}$ & 0.038992 & 2.402731 & -5.291850 \\
\hline $\mathrm{O}$ & -0.198887 & 3.081741 & 0.158620 \\
\hline $\mathrm{C}$ & -0.008446 & 4.114951 & 0.913040 \\
\hline $\mathrm{H}$ & -0.049036 & 5.098611 & 0.448040 \\
\hline $\mathrm{C}$ & -0.379146 & 4.004581 & 2.334540 \\
\hline $\mathrm{C}$ & -0.572545 & 5.171471 & 3.117420 \\
\hline $\mathrm{C}$ & -0.557417 & 2.739381 & 2.914080 \\
\hline $\mathrm{C}$ & -0.955186 & 5.034431 & 4.463640 \\
\hline $\mathrm{C}$ & -0.931048 & 2.625531 & 4.249890 \\
\hline $\mathrm{C}$ & -1.133937 & 3.772982 & 5.023640 \\
\hline $\mathrm{H}$ & -1.107635 & 5.929582 & 5.058220 \\
\hline $\mathrm{H}$ & -1.064398 & 1.640431 & 4.687030 \\
\hline $\mathrm{H}$ & -1.428507 & 3.684862 & 6.065700 \\
\hline $\mathrm{O}$ & -1.030041 & -1.854799 & 0.945330 \\
\hline $\mathrm{P}$ & 0.135349 & -1.018330 & 0.183310 \\
\hline $\mathrm{O}$ & 1.171468 & -2.190590 & -0.260020 \\
\hline $\mathrm{O}$ & 0.656100 & 0.048280 & 1.066840 \\
\hline $\mathrm{O}$ & -0.428850 & -0.593719 & -1.227040 \\
\hline $\mathrm{H}$ & -0.347639 & 0.394091 & -1.443060 \\
\hline
\end{tabular}




\begin{tabular}{|c|c|c|c|}
\hline $\mathrm{C}$ & -1.405812 & -3.172928 & 0.710850 \\
\hline $\mathrm{C}$ & -0.490523 & -4.222299 & 0.922850 \\
\hline $\mathrm{C}$ & -2.758533 & -3.406947 & 0.405930 \\
\hline $\mathrm{C}$ & -0.970914 & -5.537719 & 0.794770 \\
\hline $\mathrm{C}$ & -3.190564 & -4.737487 & 0.325440 \\
\hline $\mathrm{C}$ & -2.305974 & -5.797478 & 0.510070 \\
\hline $\mathrm{H}$ & -0.273455 & -6.360969 & 0.912930 \\
\hline $\mathrm{H}$ & -4.236414 & -4.929916 & 0.103870 \\
\hline $\mathrm{H}$ & -2.654855 & -6.822627 & 0.424420 \\
\hline $\mathrm{C}$ & 1.733928 & -3.019491 & 0.709970 \\
\hline $\mathrm{C}$ & 0.932417 & -4.014520 & 1.295830 \\
\hline $\mathrm{C}$ & 3.108188 & -2.896582 & 0.971720 \\
\hline $\mathrm{C}$ & 1.545406 & -4.881011 & 2.217410 \\
\hline $\mathrm{C}$ & 3.675887 & -3.807882 & 1.873050 \\
\hline $\mathrm{C}$ & 2.902386 & -4.781682 & 2.502560 \\
\hline $\mathrm{H}$ & 0.939846 & -5.632290 & 2.714640 \\
\hline $\mathrm{H}$ & 4.739597 & -3.738023 & 2.080640 \\
\hline $\mathrm{H}$ & 3.359156 & -5.463962 & 3.213900 \\
\hline $\mathrm{C}$ & 3.963249 & -1.849893 & 0.319530 \\
\hline $\mathrm{C}$ & 4.477450 & -0.781573 & 1.091670 \\
\hline $\mathrm{C}$ & 4.303719 & -1.956643 & -1.051280 \\
\hline $\mathrm{C}$ & 5.316740 & 0.153056 & 0.471800 \\
\hline $\mathrm{C}$ & 5.147559 & -0.995744 & -1.615860 \\
\hline $\mathrm{C}$ & 5.667220 & 0.069556 & -0.875460 \\
\hline $\mathrm{H}$ & 5.712181 & 0.977006 & 1.061950 \\
\hline $\mathrm{H}$ & 5.411359 & -1.087744 & -2.666590 \\
\hline $\mathrm{C}$ & -3.728752 & -2.290456 & 0.155920 \\
\hline $\mathrm{C}$ & -4.200121 & -2.056896 & -1.159660 \\
\hline $\mathrm{C}$ & -4.210591 & -1.503916 & 1.226420 \\
\hline $\mathrm{C}$ & -5.122511 & -1.026685 & -1.372410 \\
\hline $\mathrm{C}$ & -5.136300 & -0.489935 & 0.955980 \\
\hline $\mathrm{C}$ & -5.602650 & -0.226525 & -0.332310 \\
\hline $\mathrm{H}$ & -5.479020 & -0.848105 & -2.384120 \\
\hline $\mathrm{H}$ & -5.513190 & 0.112575 & 1.779670 \\
\hline $\mathrm{C}$ & 4.166790 & -0.604633 & 2.579020 \\
\hline $\mathrm{C}$ & 3.501711 & 0.751988 & 2.878790 \\
\hline $\mathrm{C}$ & 5.429190 & -0.802454 & 3.442030 \\
\hline $\mathrm{H}$ & 3.448329 & -1.374962 & 2.871340 \\
\hline $\mathrm{H}$ & 2.566761 & 0.846719 & 2.319620 \\
\hline $\mathrm{H}$ & 3.273371 & 0.829668 & 3.949170 \\
\hline $\mathrm{H}$ & 4.157022 & 1.592587 & 2.620120 \\
\hline $\mathrm{H}$ & 5.885009 & -1.784764 & 3.271850 \\
\hline $\mathrm{H}$ & 6.189050 & -0.043624 & 3.219670 \\
\hline $\mathrm{H}$ & 5.181780 & -0.723744 & 4.507720 \\
\hline $\mathrm{C}$ & 3.815838 & -3.105913 & -1.933350 \\
\hline $\mathrm{C}$ & 4.988267 & -3.994613 & -2.394710 \\
\hline $\mathrm{C}$ & 2.992948 & -2.604232 & -3.135140 \\
\hline $\mathrm{H}$ & 3.155317 & -3.739352 & -1.335310 \\
\hline $\mathrm{H}$ & 5.548007 & -4.389224 & -1.539190 \\
\hline $\mathrm{H}$ & 4.617676 & -4.844773 & -2.980310 \\
\hline $\mathrm{H}$ & 5.691917 & -3.437834 & -3.024770 \\
\hline $\mathrm{H}$ & 2.140179 & -2.005621 & -2.801720 \\
\hline $\mathrm{H}$ & 3.599669 & -1.991312 & -3.812580 \\
\hline
\end{tabular}




\begin{tabular}{|c|c|c|c|}
\hline $\mathrm{H}$ & 2.609787 & -3.453772 & -3.713780 \\
\hline $\mathrm{C}$ & 6.598251 & 1.099165 & -1.503070 \\
\hline $\mathrm{C}$ & 5.938272 & 1.836596 & -2.682600 \\
\hline $\mathrm{C}$ & 7.939241 & 0.469514 & -1.926820 \\
\hline $\mathrm{H}$ & 6.817252 & 1.847795 & -0.729280 \\
\hline $\mathrm{H}$ & 4.999752 & 2.310287 & -2.375220 \\
\hline $\mathrm{H}$ & 6.604332 & 2.615555 & -3.073260 \\
\hline $\mathrm{H}$ & 5.712221 & 1.149726 & -3.506770 \\
\hline $\mathrm{H}$ & 8.432430 & -0.018496 & -1.078990 \\
\hline $\mathrm{H}$ & 7.793730 & -0.286546 & -2.707650 \\
\hline $\mathrm{H}$ & 8.618561 & 1.233454 & -2.324400 \\
\hline $\mathrm{C}$ & -3.801571 & -1.755776 & 2.676910 \\
\hline $\mathrm{C}$ & -4.993842 & -2.277515 & 3.504310 \\
\hline $\mathrm{C}$ & -3.172280 & -0.512897 & 3.332320 \\
\hline $\mathrm{H}$ & -3.041362 & -2.541677 & 2.685390 \\
\hline $\mathrm{H}$ & -5.411262 & -3.190725 & 3.065220 \\
\hline $\mathrm{H}$ & -4.679472 & -2.506476 & 4.530020 \\
\hline $\mathrm{H}$ & -5.800001 & -1.536165 & 3.559450 \\
\hline $\mathrm{H}$ & -2.299960 & -0.175068 & 2.765310 \\
\hline $\mathrm{H}$ & -3.883789 & 0.319024 & 3.394540 \\
\hline $\mathrm{H}$ & -2.848760 & -0.748357 & 4.354090 \\
\hline $\mathrm{C}$ & -3.751132 & -2.893406 & -2.359890 \\
\hline $\mathrm{C}$ & -3.029881 & -2.045847 & -3.423020 \\
\hline $\mathrm{C}$ & -4.929693 & -3.671365 & -2.977730 \\
\hline $\mathrm{H}$ & -3.027883 & -3.633497 & -2.008240 \\
\hline $\mathrm{H}$ & -2.157521 & -1.548348 & -2.989590 \\
\hline $\mathrm{H}$ & -2.688762 & -2.682497 & -4.248850 \\
\hline $\mathrm{H}$ & -3.688031 & -1.277806 & -3.846450 \\
\hline $\mathrm{H}$ & -5.418303 & -4.314665 & -2.236820 \\
\hline $\mathrm{H}$ & -5.692442 & -2.994715 & -3.381180 \\
\hline $\mathrm{H}$ & -4.580183 & -4.306666 & -3.800640 \\
\hline $\mathrm{C}$ & -6.609079 & 0.889326 & -0.582310 \\
\hline $\mathrm{C}$ & -7.940679 & 0.349927 & -1.138340 \\
\hline $\mathrm{C}$ & -6.034318 & 1.987456 & -1.496700 \\
\hline $\mathrm{H}$ & -6.823779 & 1.352286 & 0.390690 \\
\hline $\mathrm{H}$ & -8.370290 & -0.406113 & -0.472030 \\
\hline $\mathrm{H}$ & -8.670239 & 1.160998 & -1.251260 \\
\hline $\mathrm{H}$ & -7.803140 & -0.112903 & -2.122820 \\
\hline $\mathrm{H}$ & -5.109998 & 2.404345 & -1.081880 \\
\hline $\mathrm{H}$ & -5.805568 & 1.593905 & -2.494340 \\
\hline $\mathrm{H}$ & -6.753667 & 2.806446 & -1.618820 \\
\hline $\mathrm{C}$ & -0.379604 & 6.479191 & 2.572830 \\
\hline $\mathrm{C}$ & -0.213223 & 7.594001 & 2.130990 \\
\hline $\mathrm{H}$ & -0.073593 & 8.577071 & 1.741650 \\
\hline $\mathrm{H}$ & -0.384168 & 1.855181 & 2.306660 \\
\hline
\end{tabular}

\section{1a allylboration TS 4}

B3LYP Energy =

$-3225.105595$

$-3223.957522$

$-3223.518239$

M06-2X Derived Solution Free Energy $(\mathrm{DCM})=$

$-3223.508811$

$1(-295.32)$ 
B3LYP/6-31G(d) Geometry

\begin{tabular}{|c|c|c|c|}
\hline B & -0.061419 & 3.449970 & 0.569060 \\
\hline $\mathrm{C}$ & -1.070689 & 3.448631 & -0.838140 \\
\hline $\mathrm{C}$ & -0.719409 & 4.681381 & -1.491080 \\
\hline $\mathrm{C}$ & 0.380011 & 4.817970 & -2.307900 \\
\hline $\mathrm{H}$ & -2.087379 & 3.424161 & -0.440090 \\
\hline $\mathrm{H}$ & -0.836859 & 2.538151 & -1.393830 \\
\hline $\mathrm{H}$ & -1.201408 & 5.589091 & -1.126210 \\
\hline $\mathrm{H}$ & 0.656992 & 5.790260 & -2.706730 \\
\hline $\mathrm{H}$ & 0.774771 & 3.951200 & -2.829240 \\
\hline $\mathrm{O}$ & -0.314529 & 4.598020 & 1.388850 \\
\hline $\mathrm{C}$ & -0.256189 & 4.185110 & 2.766490 \\
\hline $\mathrm{O}$ & -0.217759 & 2.274210 & 1.406940 \\
\hline $\mathrm{C}$ & -0.760809 & 2.697371 & 2.688230 \\
\hline $\mathrm{C}$ & 1.199851 & 4.295230 & 3.252100 \\
\hline $\mathrm{H}$ & 1.855951 & 3.616140 & 2.699840 \\
\hline $\mathrm{H}$ & 1.548352 & 5.319730 & 3.083110 \\
\hline $\mathrm{H}$ & 1.294651 & 4.077350 & 4.321610 \\
\hline $\mathrm{C}$ & -1.146019 & 5.119981 & 3.586860 \\
\hline $\mathrm{H}$ & -0.729418 & 6.132811 & 3.567370 \\
\hline $\mathrm{H}$ & -2.159289 & 5.166891 & 3.181310 \\
\hline $\mathrm{H}$ & -1.200609 & 4.798011 & 4.633430 \\
\hline $\mathrm{C}$ & -0.225889 & 1.774410 & 3.782030 \\
\hline $\mathrm{H}$ & 0.865171 & 1.735490 & 3.781520 \\
\hline $\mathrm{H}$ & -0.598650 & 0.756110 & 3.630900 \\
\hline $\mathrm{H}$ & -0.563539 & 2.113210 & 4.768410 \\
\hline $\mathrm{C}$ & -2.290549 & 2.589371 & 2.613720 \\
\hline $\mathrm{H}$ & -2.703459 & 3.287761 & 1.880740 \\
\hline $\mathrm{H}$ & -2.566889 & 1.575631 & 2.308990 \\
\hline $\mathrm{H}$ & -2.755479 & 2.793381 & 3.584510 \\
\hline $\mathrm{O}$ & 1.321061 & 3.427740 & -0.054870 \\
\hline $\mathrm{C}$ & 1.775701 & 4.499390 & -0.612130 \\
\hline $\mathrm{H}$ & 1.522942 & 5.448610 & -0.136010 \\
\hline $\mathrm{C}$ & 3.049131 & 4.466570 & -1.355930 \\
\hline $\mathrm{C}$ & 3.851822 & 5.614889 & -1.290390 \\
\hline $\mathrm{C}$ & 3.501771 & 3.344319 & -2.102070 \\
\hline $\mathrm{C}$ & 5.094722 & 5.666409 & -1.915260 \\
\hline $\mathrm{C}$ & 4.759831 & 3.415089 & -2.727470 \\
\hline $\mathrm{C}$ & 5.550321 & 4.557659 & -2.631770 \\
\hline $\mathrm{H}$ & 3.496322 & 6.472449 & -0.724000 \\
\hline $\mathrm{H}$ & 5.703942 & 6.562359 & -1.840800 \\
\hline $\mathrm{H}$ & 5.099931 & 2.558169 & -3.300100 \\
\hline $\mathrm{H}$ & 6.518611 & 4.584429 & -3.123520 \\
\hline $\mathrm{O}$ & 0.558770 & -2.270580 & -0.541330 \\
\hline $\mathrm{P}$ & -0.415080 & -1.009840 & -0.236640 \\
\hline $\mathrm{O}$ & -1.743810 & -1.709929 & 0.393220 \\
\hline $\mathrm{O}$ & -0.609520 & -0.212900 & -1.469310 \\
\hline $\mathrm{O}$ & 0.181440 & -0.290200 & 1.031850 \\
\hline $\mathrm{H}$ & 0.032670 & 0.713050 & 1.083230 \\
\hline $\mathrm{C}$ & 0.655949 & -3.420940 & 0.236680 \\
\hline $\mathrm{C}$ & -0.454621 & -4.276260 & 0.362030 \\
\hline $\mathrm{C}$ & 1.923539 & -3.754390 & 0.742530 \\
\hline $\mathrm{C}$ & -0.283321 & -5.462120 & 1.096410 \\
\hline
\end{tabular}




\begin{tabular}{|c|c|c|c|}
\hline $\mathrm{C}$ & 2.044559 & -4.959710 & 1.447990 \\
\hline $\mathrm{C}$ & 0.949879 & -5.800010 & 1.641640 \\
\hline $\mathrm{H}$ & -1.138711 & -6.115589 & 1.238080 \\
\hline $\mathrm{H}$ & 3.020269 & -5.236240 & 1.836520 \\
\hline $\mathrm{H}$ & 1.061709 & -6.721580 & 2.205750 \\
\hline $\mathrm{C}$ & -2.377890 & -2.751889 & -0.282630 \\
\hline $\mathrm{C}$ & -1.761611 & -4.014519 & -0.294420 \\
\hline $\mathrm{C}$ & -3.656830 & -2.527239 & -0.816200 \\
\hline $\mathrm{C}$ & -2.449921 & -5.069569 & -0.916650 \\
\hline $\mathrm{C}$ & -4.310131 & -3.617679 & -1.407210 \\
\hline $\mathrm{C}$ & -3.710461 & -4.874489 & -1.470470 \\
\hline $\mathrm{H}$ & -1.976701 & -6.045279 & -0.969660 \\
\hline $\mathrm{H}$ & -5.305211 & -3.467198 & -1.815210 \\
\hline $\mathrm{H}$ & -4.228231 & -5.701789 & -1.947530 \\
\hline $\mathrm{C}$ & -4.345430 & -1.195909 & -0.725530 \\
\hline $\mathrm{C}$ & -4.417010 & -0.351749 & -1.857040 \\
\hline $\mathrm{C}$ & -4.977910 & -0.817348 & 0.483000 \\
\hline $\mathrm{C}$ & -5.119530 & 0.854982 & -1.748620 \\
\hline $\mathrm{C}$ & -5.656900 & 0.403882 & 0.537120 \\
\hline $\mathrm{C}$ & -5.743239 & 1.256552 & -0.567170 \\
\hline $\mathrm{H}$ & -5.184999 & 1.505852 & -2.617430 \\
\hline $\mathrm{H}$ & -6.143180 & 0.690512 & 1.466720 \\
\hline $\mathrm{C}$ & 3.143989 & -2.913580 & 0.498600 \\
\hline $\mathrm{C}$ & 3.697260 & -2.138831 & 1.545580 \\
\hline $\mathrm{C}$ & 3.790459 & -2.963551 & -0.757570 \\
\hline $\mathrm{C}$ & 4.889350 & -1.444931 & 1.315190 \\
\hline $\mathrm{C}$ & 4.975600 & -2.240371 & -0.935640 \\
\hline $\mathrm{C}$ & 5.547710 & -1.479181 & 0.083430 \\
\hline $\mathrm{H}$ & 5.313970 & -0.855881 & 2.124010 \\
\hline $\mathrm{H}$ & 5.475570 & -2.278941 & -1.901180 \\
\hline $\mathrm{C}$ & -3.793860 & -0.728639 & -3.201680 \\
\hline $\mathrm{C}$ & -2.930940 & 0.396361 & -3.801700 \\
\hline $\mathrm{C}$ & -4.878460 & -1.174658 & -4.203660 \\
\hline $\mathrm{H}$ & -3.127270 & -1.580239 & -3.035200 \\
\hline $\mathrm{H}$ & -2.134240 & 0.679411 & -3.110290 \\
\hline $\mathrm{H}$ & -2.467630 & 0.049841 & -4.733670 \\
\hline $\mathrm{H}$ & -3.524319 & 1.285781 & -4.045210 \\
\hline $\mathrm{H}$ & -5.463890 & -2.017458 & -3.819510 \\
\hline $\mathrm{H}$ & -5.576910 & -0.356818 & -4.419390 \\
\hline $\mathrm{H}$ & -4.421760 & -1.483949 & -5.151780 \\
\hline $\mathrm{C}$ & -4.979450 & -1.715688 & 1.720390 \\
\hline $\mathrm{C}$ & -6.409050 & -2.149748 & 2.099980 \\
\hline $\mathrm{C}$ & -4.263790 & -1.059249 & 2.915750 \\
\hline $\mathrm{H}$ & -4.425770 & -2.627379 & 1.478800 \\
\hline $\mathrm{H}$ & -6.906730 & -2.650528 & 1.261970 \\
\hline $\mathrm{H}$ & -6.385971 & -2.845728 & 2.947360 \\
\hline $\mathrm{H}$ & -7.027930 & -1.292798 & 2.390720 \\
\hline $\mathrm{H}$ & -3.225640 & -0.821629 & 2.663250 \\
\hline $\mathrm{H}$ & -4.761600 & -0.132578 & 3.225230 \\
\hline $\mathrm{H}$ & -4.258020 & -1.737279 & 3.778100 \\
\hline $\mathrm{C}$ & -6.504779 & 2.574342 & -0.499420 \\
\hline $\mathrm{C}$ & -5.890999 & 3.543952 & 0.527430 \\
\hline $\mathrm{C}$ & -8.004509 & 2.355782 & -0.223030 \\
\hline
\end{tabular}




\begin{tabular}{|c|c|c|c|}
\hline $\mathrm{H}$ & -6.418989 & 3.046102 & -1.487950 \\
\hline $\mathrm{H}$ & -4.834969 & 3.735452 & 0.306680 \\
\hline $\mathrm{H}$ & -6.421119 & 4.504162 & 0.520400 \\
\hline $\mathrm{H}$ & -5.950499 & 3.137722 & 1.544150 \\
\hline $\mathrm{H}$ & -8.452379 & 1.695142 & -0.973400 \\
\hline $\mathrm{H}$ & -8.165539 & 1.899622 & 0.761040 \\
\hline $\mathrm{H}$ & -8.545349 & 3.309802 & -0.240560 \\
\hline $\mathrm{C}$ & 3.266389 & -3.804280 & -1.921380 \\
\hline $\mathrm{C}$ & 4.248929 & -4.935491 & -2.284550 \\
\hline $\mathrm{C}$ & 2.934699 & -2.940720 & -3.152720 \\
\hline $\mathrm{H}$ & 2.335329 & -4.282880 & -1.605600 \\
\hline $\mathrm{H}$ & 4.451279 & -5.577471 & -1.419740 \\
\hline $\mathrm{H}$ & 3.834509 & -5.561991 & -3.083820 \\
\hline $\mathrm{H}$ & 5.208419 & -4.538251 & -2.636370 \\
\hline $\mathrm{H}$ & 2.203730 & -2.167010 & -2.899580 \\
\hline $\mathrm{H}$ & 3.827890 & -2.446801 & -3.553240 \\
\hline $\mathrm{H}$ & 2.512109 & -3.561630 & -3.952110 \\
\hline $\mathrm{C}$ & 3.044670 & -2.044300 & 2.924530 \\
\hline $\mathrm{C}$ & 2.842870 & -0.585980 & 3.375090 \\
\hline $\mathrm{C}$ & 3.843649 & -2.832971 & 3.981260 \\
\hline $\mathrm{H}$ & 2.050040 & -2.495850 & 2.858000 \\
\hline $\mathrm{H}$ & 2.281070 & -0.023240 & 2.624920 \\
\hline $\mathrm{H}$ & 2.282430 & -0.558860 & 4.317520 \\
\hline $\mathrm{H}$ & 3.797480 & -0.074701 & 3.547460 \\
\hline $\mathrm{H}$ & 3.940649 & -3.889571 & 3.708460 \\
\hline $\mathrm{H}$ & 4.855710 & -2.426051 & 4.095840 \\
\hline $\mathrm{H}$ & 3.348140 & -2.779860 & 4.958470 \\
\hline $\mathrm{C}$ & 6.850520 & -0.723631 & -0.144670 \\
\hline $\mathrm{C}$ & 7.986990 & -1.266282 & 0.742910 \\
\hline $\mathrm{C}$ & 6.680180 & 0.795109 & 0.045590 \\
\hline $\mathrm{H}$ & 7.142010 & -0.893481 & -1.190510 \\
\hline $\mathrm{H}$ & 8.138710 & -2.339062 & 0.580410 \\
\hline $\mathrm{H}$ & 8.930000 & -0.750162 & 0.524820 \\
\hline $\mathrm{H}$ & 7.764640 & -1.118382 & 1.806480 \\
\hline $\mathrm{H}$ & 5.897530 & 1.192799 & -0.609060 \\
\hline $\mathrm{H}$ & 6.403710 & 1.038379 & 1.078250 \\
\hline $\mathrm{H}$ & 7.616701 & 1.320138 & -0.179580 \\
\hline $\mathrm{C}$ & 2.692751 & 2.184610 & -2.280650 \\
\hline $\mathrm{C}$ & 1.991910 & 1.216240 & -2.473820 \\
\hline $\mathrm{H}$ & 1.291790 & 0.407680 & -2.538700 \\
\hline
\end{tabular}

\section{1d allylboration TS 4}

B3LYP Energy =

B3LYP Free Energy =

M06-2X Derived Solution Free Energy $(\mathrm{DCM})=$

M06-2X Derived Solution Free Energy (toluene) $=$ Number of Imaginary Frequencies $=$

$-3456.178607$

$-3454.951918$

$-3454.467084$

$-3454.458013$

$1(-309.70)$

B3LYP/6-31G(d) Geometry

$\begin{array}{llll}\mathrm{B} & 2.218880 & 0.349330 & 1.431400 \\ \mathrm{C} & 2.045320 & 2.049830 & 1.110950 \\ \mathrm{C} & 3.274130 & 2.370869 & 0.440200\end{array}$




\begin{tabular}{|c|c|c|c|}
\hline $\mathrm{C}$ & 3.476120 & 2.148049 & -0.904880 \\
\hline $\mathrm{H}$ & 1.936971 & 2.493670 & 2.102690 \\
\hline $\mathrm{H}$ & 1.143360 & 2.166600 & 0.506840 \\
\hline $\mathrm{H}$ & 4.149061 & 2.552109 & 1.065540 \\
\hline $\mathrm{H}$ & 4.449470 & 2.319939 & -1.356020 \\
\hline $\mathrm{H}$ & 2.627140 & 2.138159 & -1.580740 \\
\hline $\mathrm{O}$ & 3.355880 & 0.100839 & 2.267620 \\
\hline $\mathrm{C}$ & 3.005970 & -0.946711 & 3.188930 \\
\hline $\mathrm{O}$ & 1.060360 & -0.237460 & 2.082220 \\
\hline $\mathrm{C}$ & 1.463190 & -0.712860 & 3.393900 \\
\hline $\mathrm{C}$ & 3.317659 & -2.304431 & 2.534080 \\
\hline $\mathrm{H}$ & 2.707649 & -2.464061 & 1.640110 \\
\hline $\mathrm{H}$ & 4.370679 & -2.315251 & 2.233600 \\
\hline $\mathrm{H}$ & 3.151129 & -3.140441 & 3.222250 \\
\hline $\mathrm{C}$ & 3.850470 & -0.778691 & 4.452940 \\
\hline $\mathrm{H}$ & 4.906060 & -0.946021 & 4.213100 \\
\hline $\mathrm{H}$ & 3.756660 & 0.228219 & 4.866420 \\
\hline $\mathrm{H}$ & 3.561210 & -1.503631 & 5.222930 \\
\hline $\mathrm{C}$ & 0.664729 & -1.969850 & 3.734630 \\
\hline $\mathrm{H}$ & 0.710019 & -2.708740 & 2.931300 \\
\hline $\mathrm{H}$ & -0.386601 & -1.715970 & 3.899160 \\
\hline $\mathrm{H}$ & 1.044599 & -2.427930 & 4.655510 \\
\hline $\mathrm{C}$ & 1.152470 & 0.394230 & 4.412810 \\
\hline $\mathrm{H}$ & 1.753780 & 1.288670 & 4.226260 \\
\hline $\mathrm{H}$ & 0.095940 & 0.669000 & 4.328650 \\
\hline $\mathrm{H}$ & 1.338260 & 0.061150 & 5.439880 \\
\hline $\mathrm{O}$ & 2.325640 & -0.216700 & 0.040410 \\
\hline $\mathrm{C}$ & 3.403300 & -0.028171 & -0.652770 \\
\hline $\mathrm{H}$ & 4.355640 & -0.063091 & -0.126460 \\
\hline C & 3.388500 & -0.420281 & -2.071370 \\
\hline $\mathrm{C}$ & 4.609390 & -0.628881 & -2.767220 \\
\hline $\mathrm{C}$ & 2.166080 & -0.601200 & -2.735070 \\
\hline $\mathrm{C}$ & 4.560670 & -1.026771 & -4.117470 \\
\hline $\mathrm{C}$ & 2.142150 & -0.991360 & -4.070490 \\
\hline $\mathrm{C}$ & 3.340620 & -1.207121 & -4.760270 \\
\hline $\mathrm{H}$ & 5.493560 & -1.191411 & -4.647360 \\
\hline $\mathrm{H}$ & 1.189210 & -1.127450 & -4.573080 \\
\hline $\mathrm{H}$ & 3.323290 & -1.514041 & -5.802450 \\
\hline $\mathrm{O}$ & -2.543030 & -1.023449 & -1.036720 \\
\hline $\mathrm{P}$ & -1.759110 & 0.142051 & -0.220690 \\
\hline $\mathrm{O}$ & -2.961050 & 1.172401 & 0.151270 \\
\hline $\mathrm{O}$ & -0.642370 & 0.670140 & -1.035190 \\
\hline $\mathrm{O}$ & -1.421930 & -0.424390 & 1.212300 \\
\hline $\mathrm{H}$ & -0.448670 & -0.345580 & 1.487460 \\
\hline $\mathrm{C}$ & -3.871290 & -1.404809 & -0.882490 \\
\hline $\mathrm{C}$ & -4.909470 & -0.493759 & -1.158900 \\
\hline $\mathrm{C}$ & -4.117941 & -2.757789 & -0.589070 \\
\hline $\mathrm{C}$ & -6.228100 & -0.980208 & -1.113660 \\
\hline $\mathrm{C}$ & -5.448901 & -3.196099 & -0.593260 \\
\hline $\mathrm{C}$ & -6.499081 & -2.316668 & -0.846300 \\
\hline $\mathrm{H}$ & -7.045610 & -0.286468 & -1.282870 \\
\hline $\mathrm{H}$ & -5.650311 & -4.242328 & -0.381730 \\
\hline $\mathrm{H}$ & -7.525971 & -2.670328 & -0.825770 \\
\hline
\end{tabular}




\begin{tabular}{|c|c|c|c|}
\hline $\mathrm{C}$ & -3.729680 & 1.733931 & -0.867490 \\
\hline $\mathrm{C}$ & -4.684900 & 0.931021 & -1.514840 \\
\hline $\mathrm{C}$ & -3.593359 & 3.108701 & -1.120050 \\
\hline $\mathrm{C}$ & -5.495940 & 1.544162 & -2.485470 \\
\hline $\mathrm{C}$ & -4.451299 & 3.676601 & -2.071970 \\
\hline $\mathrm{C}$ & -5.384239 & 2.902391 & -2.759920 \\
\hline $\mathrm{H}$ & -6.213780 & 0.937892 & -3.029010 \\
\hline $\mathrm{H}$ & -4.371879 & 4.740931 & -2.272840 \\
\hline $\mathrm{H}$ & -6.024309 & 3.359412 & -3.509310 \\
\hline $\mathrm{C}$ & -2.583449 & 3.962591 & -0.410720 \\
\hline $\mathrm{C}$ & -1.472239 & 4.473210 & -1.122430 \\
\hline $\mathrm{C}$ & -2.764799 & 4.304001 & 0.951960 \\
\hline $\mathrm{C}$ & -0.571139 & 5.310230 & -0.452070 \\
\hline $\mathrm{C}$ & -1.833719 & 5.144571 & 1.568930 \\
\hline $\mathrm{C}$ & -0.727989 & 5.661430 & 0.888380 \\
\hline $\mathrm{H}$ & 0.284901 & 5.703200 & -0.996250 \\
\hline $\mathrm{H}$ & -1.982359 & 5.408391 & 2.613240 \\
\hline $\mathrm{C}$ & -3.015491 & -3.720279 & -0.258990 \\
\hline $\mathrm{C}$ & -2.870061 & -4.181569 & 1.072860 \\
\hline $\mathrm{C}$ & -2.154501 & -4.204259 & -1.269370 \\
\hline $\mathrm{C}$ & -1.853091 & -5.097369 & 1.362220 \\
\hline $\mathrm{C}$ & -1.154541 & -5.120040 & -0.922700 \\
\hline $\mathrm{C}$ & -0.979782 & -5.578750 & 0.383250 \\
\hline $\mathrm{H}$ & -1.745021 & -5.447629 & 2.385960 \\
\hline $\mathrm{H}$ & -0.492191 & -5.495330 & -1.699750 \\
\hline $\mathrm{C}$ & -1.214369 & 4.162470 & -2.598030 \\
\hline $\mathrm{C}$ & 0.157281 & 3.499360 & -2.824750 \\
\hline $\mathrm{C}$ & -1.367589 & 5.424890 & -3.470180 \\
\hline $\mathrm{H}$ & -1.966879 & 3.443171 & -2.931620 \\
\hline $\mathrm{H}$ & 0.223031 & 2.563990 & -2.262140 \\
\hline $\mathrm{H}$ & 0.292571 & 3.272250 & -3.889650 \\
\hline $\mathrm{H}$ & 0.982101 & 4.155220 & -2.520790 \\
\hline $\mathrm{H}$ & -2.357929 & 5.879711 & -3.351890 \\
\hline $\mathrm{H}$ & -0.622289 & 6.185480 & -3.208120 \\
\hline $\mathrm{H}$ & -1.232529 & 5.177620 & -4.530260 \\
\hline $\mathrm{C}$ & -3.962529 & 3.819631 & 1.769020 \\
\hline $\mathrm{C}$ & -4.871179 & 4.994841 & 2.181930 \\
\hline $\mathrm{C}$ & -3.529729 & 2.995091 & 2.996260 \\
\hline $\mathrm{H}$ & -4.564659 & 3.161591 & 1.136770 \\
\hline $\mathrm{H}$ & -5.213979 & 5.558401 & 1.306790 \\
\hline $\mathrm{H}$ & -5.755079 & 4.626612 & 2.716860 \\
\hline $\mathrm{H}$ & -4.348429 & 5.694511 & 2.844810 \\
\hline $\mathrm{H}$ & -2.915780 & 2.140831 & 2.696090 \\
\hline $\mathrm{H}$ & -2.953359 & 3.600601 & 3.706220 \\
\hline $\mathrm{H}$ & -4.410569 & 2.613941 & 3.527470 \\
\hline $\mathrm{C}$ & 0.268072 & 6.589670 & 1.572010 \\
\hline $\mathrm{C}$ & 0.947651 & 5.921930 & 2.781670 \\
\hline $\mathrm{C}$ & -0.383888 & 7.926590 & 1.974280 \\
\hline $\mathrm{H}$ & 1.053462 & 6.815150 & 0.837520 \\
\hline $\mathrm{H}$ & 1.441741 & 4.988910 & 2.490130 \\
\hline $\mathrm{H}$ & 1.701812 & 6.588060 & 3.218200 \\
\hline $\mathrm{H}$ & 0.220321 & 5.684130 & 3.566890 \\
\hline $\mathrm{H}$ & -0.827828 & 8.427040 & 1.106690 \\
\hline
\end{tabular}




\begin{tabular}{|c|c|c|c|}
\hline $\mathrm{H}$ & -1.179388 & 7.773900 & 2.713470 \\
\hline $\mathrm{H}$ & 0.357712 & 8.602820 & 2.416800 \\
\hline $\mathrm{C}$ & -2.307961 & -3.805589 & -2.736420 \\
\hline $\mathrm{C}$ & -2.738451 & -5.010879 & -3.596550 \\
\hline $\mathrm{C}$ & -1.033491 & -3.150430 & -3.299940 \\
\hline $\mathrm{H}$ & -3.108101 & -3.063319 & -2.805270 \\
\hline $\mathrm{H}$ & -3.670951 & -5.450539 & -3.225020 \\
\hline $\mathrm{H}$ & -2.898641 & -4.702539 & -4.636980 \\
\hline $\mathrm{H}$ & -1.975222 & -5.798159 & -3.595400 \\
\hline $\mathrm{H}$ & -0.758571 & -2.268040 & -2.715010 \\
\hline $\mathrm{H}$ & -0.183931 & -3.843530 & -3.294160 \\
\hline $\mathrm{H}$ & -1.197861 & -2.837110 & -4.338740 \\
\hline $\mathrm{C}$ & -3.791381 & -3.731669 & 2.208950 \\
\hline $\mathrm{C}$ & -3.023001 & -3.016439 & 3.334930 \\
\hline $\mathrm{C}$ & -4.616911 & -4.909339 & 2.763450 \\
\hline $\mathrm{H}$ & -4.500641 & -3.004749 & 1.805200 \\
\hline $\mathrm{H}$ & -2.489651 & -2.145929 & 2.942190 \\
\hline $\mathrm{H}$ & -3.718891 & -2.673249 & 4.110490 \\
\hline $\mathrm{H}$ & -2.293251 & -3.679499 & 3.814670 \\
\hline $\mathrm{H}$ & -5.203801 & -5.393909 & 1.974640 \\
\hline $\mathrm{H}$ & -3.974392 & -5.675209 & 3.214100 \\
\hline $\mathrm{H}$ & -5.311491 & -4.560399 & 3.537340 \\
\hline $\mathrm{C}$ & 0.114968 & -6.585080 & 0.714840 \\
\hline $\mathrm{C}$ & -0.469892 & -7.926110 & 1.198080 \\
\hline $\mathrm{C}$ & 1.127018 & -6.026650 & 1.732310 \\
\hline $\mathrm{H}$ & 0.662688 & -6.782930 & -0.216810 \\
\hline $\mathrm{H}$ & -1.156082 & -8.348720 & 0.455960 \\
\hline $\mathrm{H}$ & 0.329258 & -8.655060 & 1.379730 \\
\hline $\mathrm{H}$ & -1.026922 & -7.802070 & 2.134420 \\
\hline $\mathrm{H}$ & 1.575659 & -5.094080 & 1.372960 \\
\hline $\mathrm{H}$ & 0.648918 & -5.818410 & 2.696950 \\
\hline $\mathrm{H}$ & 1.934388 & -6.747380 & 1.910290 \\
\hline $\mathrm{C}$ & 5.871270 & -0.444151 & -2.133280 \\
\hline $\mathrm{C}$ & 6.956700 & -0.292082 & -1.603400 \\
\hline $\mathrm{H}$ & 1.243540 & -0.417100 & -2.191270 \\
\hline $\mathrm{C}$ & 8.226320 & -0.119912 & -0.980010 \\
\hline $\mathrm{C}$ & 8.316640 & 0.137448 & 0.403170 \\
\hline $\mathrm{C}$ & 9.412260 & -0.207252 & -1.736830 \\
\hline $\mathrm{C}$ & 9.560100 & 0.301748 & 1.006750 \\
\hline $\mathrm{H}$ & 7.406130 & 0.202878 & 0.991250 \\
\hline $\mathrm{C}$ & 10.650810 & -0.041083 & -1.123940 \\
\hline $\mathrm{H}$ & 9.346550 & -0.405752 & -2.802140 \\
\hline $\mathrm{C}$ & 10.729890 & 0.213517 & 0.247650 \\
\hline $\mathrm{H}$ & 9.616950 & 0.498818 & 2.073780 \\
\hline $\mathrm{H}$ & 11.557920 & -0.110783 & -1.718060 \\
\hline $\mathrm{H}$ & 11.698480 & 0.342227 & 0.722770 \\
\hline
\end{tabular}

1a allylboration TS 5 (TS-1Si-B)

B3LYP Energy =

$-3225.103321$

$-3223.955276$

B3LYP Free Energy =

$-3223.523186$

M06-2X Derived Solution Free Energy $(\mathrm{DCM})=$

$-3223.514603$ 
B3LYP/6-31G(d) Geometry

\begin{tabular}{|c|c|c|c|}
\hline $\mathrm{O}$ & 0.090801 & 4.500190 & -2.232410 \\
\hline B & 0.578910 & 3.441230 & -1.425720 \\
\hline $\mathrm{O}$ & 0.911690 & 3.912700 & -0.031460 \\
\hline $\mathrm{O}$ & -0.461220 & 2.395630 & -1.395100 \\
\hline $\mathrm{C}$ & 1.282830 & 3.064680 & 0.877710 \\
\hline $\mathrm{C}$ & -1.574950 & 2.845070 & -2.223580 \\
\hline $\mathrm{C}$ & 1.681850 & 3.557210 & 2.213370 \\
\hline $\mathrm{C}$ & 2.266851 & 4.826740 & 2.459330 \\
\hline $\mathrm{C}$ & 1.422480 & 2.698220 & 3.292360 \\
\hline $\mathrm{C}$ & 1.712260 & 3.077900 & 4.600300 \\
\hline $\mathrm{C}$ & 2.552181 & 5.191750 & 3.788030 \\
\hline $\mathrm{C}$ & 2.275901 & 4.331340 & 4.847320 \\
\hline $\mathrm{C}$ & -1.338229 & 4.400970 & -2.283950 \\
\hline $\mathrm{C}$ & -1.929319 & 5.149450 & -1.074740 \\
\hline $\mathrm{C}$ & -1.819469 & 5.067210 & -3.575150 \\
\hline $\mathrm{C}$ & -2.878950 & 2.424211 & -1.550440 \\
\hline $\mathrm{C}$ & -1.433360 & 2.170850 & -3.596470 \\
\hline $\mathrm{C}$ & 2.111160 & 2.895410 & -2.025770 \\
\hline $\mathrm{C}$ & 2.635840 & 1.983310 & -1.045820 \\
\hline $\mathrm{C}$ & 3.186800 & 2.399800 & 0.148270 \\
\hline $\mathrm{H}$ & 0.825040 & 2.075580 & 0.879550 \\
\hline $\mathrm{H}$ & 1.495890 & 2.400570 & 5.421260 \\
\hline $\mathrm{H}$ & 2.999481 & 6.163410 & 3.972230 \\
\hline $\mathrm{H}$ & 2.504581 & 4.639480 & 5.863760 \\
\hline $\mathrm{H}$ & -1.623169 & 4.694370 & -0.129040 \\
\hline $\mathrm{H}$ & -1.552669 & 6.177060 & -1.086680 \\
\hline $\mathrm{H}$ & -3.024179 & 5.182071 & -1.109530 \\
\hline $\mathrm{H}$ & -1.601949 & 6.139560 & -3.532210 \\
\hline $\mathrm{H}$ & -1.311129 & 4.657980 & -4.451170 \\
\hline $\mathrm{H}$ & -2.901369 & 4.946461 & -3.706670 \\
\hline $\mathrm{H}$ & -2.962420 & 1.335111 & -1.514590 \\
\hline $\mathrm{H}$ & -2.936090 & 2.795921 & -0.524920 \\
\hline $\mathrm{H}$ & -3.741990 & 2.806791 & -2.107200 \\
\hline $\mathrm{H}$ & -0.521860 & 2.501710 & -4.103070 \\
\hline $\mathrm{H}$ & -1.379380 & 1.085370 & -3.468340 \\
\hline $\mathrm{H}$ & -2.288380 & 2.392681 & -4.244090 \\
\hline $\mathrm{H}$ & 1.890110 & 2.443400 & -2.994720 \\
\hline $\mathrm{H}$ & 2.683430 & 3.819540 & -2.139130 \\
\hline $\mathrm{H}$ & 2.379070 & 0.928220 & -1.137640 \\
\hline $\mathrm{H}$ & 3.617630 & 3.394650 & 0.224820 \\
\hline $\mathrm{H}$ & 3.514750 & 1.668730 & 0.878720 \\
\hline $\mathrm{C}$ & -4.193920 & -1.631769 & 0.388050 \\
\hline $\mathrm{C}$ & -4.384210 & -0.968999 & 1.623340 \\
\hline $\mathrm{C}$ & -5.243040 & 0.135261 & 1.670430 \\
\hline $\mathrm{C}$ & -5.928250 & 0.604041 & 0.549280 \\
\hline $\mathrm{C}$ & -5.721520 & -0.063809 & -0.660680 \\
\hline $\mathrm{C}$ & -4.874050 & -1.172069 & -0.769660 \\
\hline $\mathrm{O}$ & -1.359150 & -1.710110 & 0.859760 \\
\hline $\mathrm{P}$ & -0.106010 & -0.950820 & 0.167270 \\
\hline $\mathrm{C}$ & -1.967490 & -2.899970 & 0.470300 \\
\hline
\end{tabular}




\begin{tabular}{|c|c|c|c|}
\hline $\mathrm{O}$ & 0.597440 & -0.122070 & 1.175790 \\
\hline $\mathrm{O}$ & 0.730760 & -2.161820 & -0.517470 \\
\hline $\mathrm{C}$ & -1.222470 & -4.094240 & 0.410010 \\
\hline $\mathrm{C}$ & -3.364990 & -2.880059 & 0.307360 \\
\hline $\mathrm{C}$ & 1.174180 & -3.249540 & 0.236720 \\
\hline $\mathrm{C}$ & 0.233230 & -4.188770 & 0.692610 \\
\hline $\mathrm{C}$ & -4.009800 & -4.101379 & 0.062950 \\
\hline $\mathrm{C}$ & -1.917521 & -5.279830 & 0.111080 \\
\hline $\mathrm{C}$ & -3.296401 & -5.290499 & -0.053140 \\
\hline $\mathrm{C}$ & 2.558670 & -3.424100 & 0.398300 \\
\hline $\mathrm{C}$ & 0.719999 & -5.307130 & 1.392630 \\
\hline $\mathrm{C}$ & 2.990540 & -4.581170 & 1.062640 \\
\hline $\mathrm{C}$ & 2.083239 & -5.506380 & 1.572820 \\
\hline $\mathrm{C}$ & 3.577690 & -2.444370 & -0.103620 \\
\hline $\mathrm{C}$ & 4.353030 & -1.708560 & 0.825120 \\
\hline $\mathrm{C}$ & 5.360960 & -0.865010 & 0.340800 \\
\hline $\mathrm{C}$ & 5.632540 & -0.723330 & -1.020480 \\
\hline $\mathrm{C}$ & 4.848750 & -1.455660 & -1.916410 \\
\hline $\mathrm{C}$ & 3.830150 & -2.314840 & -1.491430 \\
\hline $\mathrm{O}$ & -0.619150 & -0.215490 & -1.130710 \\
\hline $\mathrm{H}$ & -5.400760 & 0.637941 & 2.622260 \\
\hline $\mathrm{H}$ & -6.243730 & 0.280231 & -1.550080 \\
\hline $\mathrm{H}$ & -5.090400 & -4.100859 & -0.044800 \\
\hline $\mathrm{H}$ & -1.354151 & -6.201430 & 0.007310 \\
\hline $\mathrm{H}$ & -3.813081 & -6.221149 & -0.269240 \\
\hline $\mathrm{H}$ & 0.010269 & -6.020430 & 1.798860 \\
\hline $\mathrm{H}$ & 4.058030 & -4.739820 & 1.183620 \\
\hline $\mathrm{H}$ & 2.438999 & -6.382190 & 2.107900 \\
\hline $\mathrm{H}$ & 5.960890 & -0.300170 & 1.051410 \\
\hline $\mathrm{H}$ & 5.045690 & -1.370450 & -2.982150 \\
\hline $\mathrm{H}$ & -0.494880 & 0.797410 & -1.154240 \\
\hline $\mathrm{C}$ & -3.746890 & -1.447079 & 2.928290 \\
\hline $\mathrm{C}$ & -4.818050 & -1.948769 & 3.917830 \\
\hline $\mathrm{C}$ & -2.860050 & -0.366299 & 3.574940 \\
\hline $\mathrm{H}$ & -3.101220 & -2.299559 & 2.703220 \\
\hline $\mathrm{H}$ & -5.425170 & -2.746399 & 3.475060 \\
\hline $\mathrm{H}$ & -4.345160 & -2.344569 & 4.824830 \\
\hline $\mathrm{H}$ & -5.496200 & -1.142459 & 4.220810 \\
\hline $\mathrm{H}$ & -2.059620 & -0.055950 & 2.897190 \\
\hline $\mathrm{H}$ & -3.440780 & 0.521931 & 3.851480 \\
\hline $\mathrm{H}$ & -2.397320 & -0.755739 & 4.490050 \\
\hline $\mathrm{C}$ & -4.734070 & -1.843449 & -2.139510 \\
\hline $\mathrm{C}$ & -4.078120 & -0.914739 & -3.177810 \\
\hline $\mathrm{C}$ & -6.085750 & -2.376059 & -2.654690 \\
\hline $\mathrm{H}$ & -4.070440 & -2.704449 & -2.032960 \\
\hline $\mathrm{H}$ & -3.072830 & -0.624829 & -2.857260 \\
\hline $\mathrm{H}$ & -3.991160 & -1.426389 & -4.144190 \\
\hline $\mathrm{H}$ & -4.662870 & -0.000879 & -3.335460 \\
\hline $\mathrm{H}$ & -6.548030 & -3.061619 & -1.935260 \\
\hline $\mathrm{H}$ & -6.797970 & -1.563409 & -2.839580 \\
\hline $\mathrm{H}$ & -5.948250 & -2.916749 & -3.598790 \\
\hline $\mathrm{C}$ & -6.904290 & 1.768241 & 0.668420 \\
\hline $\mathrm{C}$ & -8.358180 & 1.301981 & 0.456260 \\
\hline
\end{tabular}




\begin{tabular}{|c|c|c|c|}
\hline $\mathrm{C}$ & -6.559950 & 2.935851 & -0.273140 \\
\hline $\mathrm{H}$ & -6.828730 & 2.145051 & 1.697730 \\
\hline $\mathrm{H}$ & -8.621540 & 0.497881 & 1.152040 \\
\hline $\mathrm{H}$ & -9.058120 & 2.132031 & 0.611210 \\
\hline $\mathrm{H}$ & -8.507960 & 0.924301 & -0.562320 \\
\hline $\mathrm{H}$ & -5.543380 & 3.302151 & -0.096280 \\
\hline $\mathrm{H}$ & -6.629650 & 2.636461 & -1.325730 \\
\hline $\mathrm{H}$ & -7.255280 & 3.770021 & -0.120570 \\
\hline $\mathrm{C}$ & 6.770360 & 0.170330 & -1.497820 \\
\hline $\mathrm{C}$ & 6.304860 & 1.232230 & -2.509930 \\
\hline $\mathrm{C}$ & 7.933110 & -0.664221 & -2.070620 \\
\hline $\mathrm{H}$ & 7.152090 & 0.702280 & -0.615500 \\
\hline $\mathrm{H}$ & 5.493960 & 1.842220 & -2.098800 \\
\hline $\mathrm{H}$ & 7.134610 & 1.897540 & -2.777110 \\
\hline $\mathrm{H}$ & 5.941160 & 0.771490 & -3.436000 \\
\hline $\mathrm{H}$ & 8.299540 & -1.390541 & -1.336790 \\
\hline $\mathrm{H}$ & 7.617900 & -1.220391 & -2.961640 \\
\hline $\mathrm{H}$ & 8.770400 & -0.017091 & -2.358930 \\
\hline $\mathrm{C}$ & 4.140250 & -1.793050 & 2.338330 \\
\hline $\mathrm{C}$ & 3.720670 & -0.437850 & 2.937480 \\
\hline $\mathrm{C}$ & 5.381590 & -2.355510 & 3.057930 \\
\hline $\mathrm{H}$ & 3.315200 & -2.483890 & 2.528730 \\
\hline $\mathrm{H}$ & 2.797090 & -0.083360 & 2.470870 \\
\hline $\mathrm{H}$ & 3.541840 & -0.539460 & 4.015160 \\
\hline $\mathrm{H}$ & 4.498230 & 0.324550 & 2.805170 \\
\hline $\mathrm{H}$ & 5.662340 & -3.340320 & 2.667060 \\
\hline $\mathrm{H}$ & 6.248940 & -1.694930 & 2.942160 \\
\hline $\mathrm{H}$ & 5.185730 & -2.460440 & 4.131870 \\
\hline $\mathrm{C}$ & 3.071020 & -3.120100 & -2.546080 \\
\hline $\mathrm{C}$ & 4.007750 & -4.098550 & -3.282850 \\
\hline $\mathrm{C}$ & 2.321970 & -2.212930 & -3.540660 \\
\hline $\mathrm{H}$ & 2.318790 & -3.728190 & -2.036680 \\
\hline $\mathrm{H}$ & 4.509620 & -4.772760 & -2.579720 \\
\hline $\mathrm{H}$ & 3.439240 & -4.710300 & -3.993860 \\
\hline $\mathrm{H}$ & 4.783870 & -3.568070 & -3.847100 \\
\hline $\mathrm{H}$ & 1.617600 & -1.557310 & -3.019870 \\
\hline $\mathrm{H}$ & 3.014160 & -1.587320 & -4.116740 \\
\hline $\mathrm{H}$ & 1.754080 & -2.820700 & -4.255450 \\
\hline $\mathrm{C}$ & 2.614311 & 5.741370 & 1.418200 \\
\hline $\mathrm{C}$ & 2.965851 & 6.556590 & 0.595800 \\
\hline $\mathrm{H}$ & 3.231421 & 7.259890 & -0.160510 \\
\hline $\mathrm{H}$ & 0.980330 & 1.726860 & 3.087680 \\
\hline
\end{tabular}

\section{1a allylboration TS 6}

B3LYP Energy $=$

B3LYP Free Energy =

M06-2X Derived Solution Free Energy $(\mathrm{DCM})=$

$-3225.104997$

$-3223.956478$

$-3223.520948$

$-3223.512782$

M06-2X Derived Solution Free Energy (toluene) $=$

$1(-270.81)$

B3LYP/6-31G(d) Geometry

$\mathrm{O}$

0.384492

4.140840

$-2.717160$ 


\begin{tabular}{|c|c|c|c|}
\hline B & 0.606202 & 3.338920 & -1.567200 \\
\hline $\mathrm{O}$ & 0.439462 & 4.187670 & -0.308900 \\
\hline $\mathrm{O}$ & -0.372199 & 2.242080 & -1.599800 \\
\hline $\mathrm{C}$ & 0.589872 & 3.722000 & 0.888130 \\
\hline $\mathrm{C}$ & -1.208679 & 2.417491 & -2.786520 \\
\hline $\mathrm{C}$ & 0.582172 & 4.698890 & 1.998140 \\
\hline $\mathrm{C}$ & 0.653623 & 6.071270 & 1.719760 \\
\hline $\mathrm{C}$ & 0.463482 & 4.266180 & 3.344520 \\
\hline $\mathrm{C}$ & 0.422753 & 5.227620 & 4.369810 \\
\hline $\mathrm{C}$ & 0.613283 & 7.009010 & 2.746640 \\
\hline $\mathrm{C}$ & 0.498303 & 6.585440 & 4.074560 \\
\hline $\mathrm{C}$ & -0.963358 & 3.929711 & -3.154780 \\
\hline $\mathrm{C}$ & -1.890348 & 4.898011 & -2.395360 \\
\hline $\mathrm{C}$ & -1.035018 & 4.241881 & -4.652140 \\
\hline $\mathrm{C}$ & -2.649499 & 2.077801 & -2.410410 \\
\hline $\mathrm{C}$ & -0.691169 & 1.458740 & -3.869520 \\
\hline $\mathrm{C}$ & 2.252962 & 2.847989 & -1.492540 \\
\hline $\mathrm{C}$ & 2.527631 & 2.360719 & -0.161350 \\
\hline $\mathrm{C}$ & 2.715952 & 3.192599 & 0.918640 \\
\hline $\mathrm{H}$ & 0.254841 & 2.708670 & 1.109710 \\
\hline $\mathrm{H}$ & 0.728493 & 6.384510 & 0.683780 \\
\hline $\mathrm{H}$ & 0.330342 & 4.890440 & 5.397290 \\
\hline $\mathrm{H}$ & 0.666394 & 8.068750 & 2.513980 \\
\hline $\mathrm{H}$ & 0.464414 & 7.314190 & 4.879580 \\
\hline $\mathrm{H}$ & -1.888228 & 4.702761 & -1.319900 \\
\hline $\mathrm{H}$ & -1.525087 & 5.918321 & -2.550280 \\
\hline $\mathrm{H}$ & -2.922828 & 4.843402 & -2.758150 \\
\hline $\mathrm{H}$ & -0.842357 & 5.308501 & -4.808180 \\
\hline $\mathrm{H}$ & -0.285398 & 3.681760 & -5.215440 \\
\hline $\mathrm{H}$ & -2.026728 & 4.014111 & -5.060620 \\
\hline $\mathrm{H}$ & -2.732579 & 1.027191 & -2.120940 \\
\hline $\mathrm{H}$ & -2.993799 & 2.682662 & -1.568800 \\
\hline $\mathrm{H}$ & -3.322159 & 2.247802 & -3.259460 \\
\hline $\mathrm{H}$ & 0.334021 & 1.711590 & -4.156450 \\
\hline $\mathrm{H}$ & -0.697630 & 0.432500 & -3.491500 \\
\hline $\mathrm{H}$ & -1.318989 & 1.492111 & -4.766350 \\
\hline $\mathrm{H}$ & 2.356291 & 2.090109 & -2.272780 \\
\hline $\mathrm{H}$ & 2.800832 & 3.756619 & -1.758300 \\
\hline $\mathrm{H}$ & 2.350211 & 1.307839 & 0.051060 \\
\hline $\mathrm{H}$ & 3.029502 & 4.222339 & 0.769210 \\
\hline $\mathrm{H}$ & 2.859881 & 2.780799 & 1.912210 \\
\hline $\mathrm{C}$ & -3.947421 & -1.976858 & 0.347110 \\
\hline $\mathrm{C}$ & -4.272280 & -1.222918 & 1.497930 \\
\hline $\mathrm{C}$ & -5.183650 & -0.167387 & 1.378290 \\
\hline $\mathrm{C}$ & -5.789130 & 0.167333 & 0.166830 \\
\hline $\mathrm{C}$ & -5.453000 & -0.592257 & -0.957080 \\
\hline $\mathrm{C}$ & -4.550921 & -1.660148 & -0.897030 \\
\hline $\mathrm{O}$ & -1.159521 & -1.796369 & 0.982830 \\
\hline $\mathrm{P}$ & 0.049170 & -0.983520 & 0.270300 \\
\hline $\mathrm{C}$ & -1.650681 & -3.058369 & 0.660250 \\
\hline $\mathrm{O}$ & 0.695100 & -0.068790 & 1.240590 \\
\hline $\mathrm{O}$ & 0.963189 & -2.172740 & -0.353250 \\
\hline $\mathrm{C}$ & -0.810302 & -4.188049 & 0.715050 \\
\hline
\end{tabular}




\begin{tabular}{|c|c|c|c|}
\hline $\mathrm{C}$ & -3.036091 & -3.165348 & 0.438810 \\
\hline $\mathrm{C}$ & 1.500359 & -3.152851 & 0.482910 \\
\hline $\mathrm{C}$ & 0.644538 & -4.134650 & 1.011210 \\
\hline $\mathrm{C}$ & -3.575262 & -4.450078 & 0.284070 \\
\hline $\mathrm{C}$ & -1.400502 & -5.447419 & 0.505410 \\
\hline $\mathrm{C}$ & -2.767983 & -5.583829 & 0.305170 \\
\hline $\mathrm{C}$ & 2.893149 & -3.181021 & 0.663370 \\
\hline $\mathrm{C}$ & 1.226388 & -5.147420 & 1.794160 \\
\hline $\mathrm{C}$ & 3.424248 & -4.240002 & 1.413450 \\
\hline $\mathrm{C}$ & 2.601058 & -5.205361 & 1.988950 \\
\hline $\mathrm{C}$ & 3.811869 & -2.138662 & 0.097930 \\
\hline $\mathrm{C}$ & 4.491170 & -1.258192 & 0.974420 \\
\hline $\mathrm{C}$ & 5.396520 & -0.336452 & 0.433500 \\
\hline $\mathrm{C}$ & 5.660800 & -0.256863 & -0.933920 \\
\hline $\mathrm{C}$ & 4.975600 & -1.135272 & -1.777850 \\
\hline $\mathrm{C}$ & 4.058709 & -2.074392 & -1.295450 \\
\hline $\mathrm{O}$ & -0.516990 & -0.338390 & -1.053440 \\
\hline $\mathrm{H}$ & -5.443500 & 0.406253 & 2.265460 \\
\hline $\mathrm{H}$ & -5.914270 & -0.354867 & -1.912760 \\
\hline $\mathrm{H}$ & -4.646412 & -4.545258 & 0.132330 \\
\hline $\mathrm{H}$ & -0.763433 & -6.325860 & 0.496010 \\
\hline $\mathrm{H}$ & -3.202333 & -6.568678 & 0.159300 \\
\hline $\mathrm{H}$ & 0.582997 & -5.890820 & 2.253750 \\
\hline $\mathrm{H}$ & 4.500358 & -4.289212 & 1.550260 \\
\hline $\mathrm{H}$ & 3.031757 & -6.002261 & 2.588520 \\
\hline $\mathrm{H}$ & 5.921630 & 0.340137 & 1.104250 \\
\hline $\mathrm{H}$ & 5.169680 & -1.099662 & -2.847060 \\
\hline $\mathrm{H}$ & -0.389330 & 0.667280 & -1.148790 \\
\hline $\mathrm{C}$ & -3.720701 & -1.557848 & 2.883150 \\
\hline $\mathrm{C}$ & -4.837121 & -2.073228 & 3.813590 \\
\hline $\mathrm{C}$ & -2.975030 & -0.370258 & 3.519050 \\
\hline $\mathrm{H}$ & -2.997661 & -2.370538 & 2.775150 \\
\hline $\mathrm{H}$ & -5.339731 & -2.947327 & 3.384420 \\
\hline $\mathrm{H}$ & -4.422061 & -2.363138 & 4.786650 \\
\hline $\mathrm{H}$ & -5.598800 & -1.304897 & 3.991470 \\
\hline $\mathrm{H}$ & -2.157080 & -0.029139 & 2.877790 \\
\hline $\mathrm{H}$ & -3.642950 & 0.480222 & 3.699580 \\
\hline $\mathrm{H}$ & -2.551950 & -0.665019 & 4.487570 \\
\hline $\mathrm{C}$ & -4.276701 & -2.450828 & -2.179970 \\
\hline $\mathrm{C}$ & -3.642841 & -1.582678 & -3.281890 \\
\hline $\mathrm{C}$ & -5.551391 & -3.147477 & -2.696460 \\
\hline $\mathrm{H}$ & -3.552541 & -3.235898 & -1.950110 \\
\hline $\mathrm{H}$ & -2.690300 & -1.162679 & -2.945330 \\
\hline $\mathrm{H}$ & -3.449881 & -2.187538 & -4.176430 \\
\hline $\mathrm{H}$ & -4.296010 & -0.752958 & -3.576820 \\
\hline $\mathrm{H}$ & -5.989172 & -3.802047 & -1.934160 \\
\hline $\mathrm{H}$ & -6.318771 & -2.420017 & -2.985800 \\
\hline $\mathrm{H}$ & -5.323432 & -3.758567 & -3.578140 \\
\hline $\mathrm{C}$ & -6.806549 & 1.298923 & 0.093510 \\
\hline $\mathrm{C}$ & -8.211069 & 0.774714 & -0.263310 \\
\hline $\mathrm{C}$ & -6.373669 & 2.415103 & -0.874400 \\
\hline $\mathrm{H}$ & -6.867529 & 1.741243 & 1.097400 \\
\hline $\mathrm{H}$ & -8.539600 & 0.007674 & 0.446530 \\
\hline
\end{tabular}




\begin{tabular}{|c|c|c|c|}
\hline $\mathrm{H}$ & -8.944159 & 1.590344 & -0.249480 \\
\hline $\mathrm{H}$ & -8.225980 & 0.330054 & -1.265600 \\
\hline $\mathrm{H}$ & -5.395159 & 2.821603 & -0.597540 \\
\hline $\mathrm{H}$ & -6.302069 & 2.045483 & -1.904190 \\
\hline $\mathrm{H}$ & -7.100018 & 3.236814 & -0.866160 \\
\hline $\mathrm{C}$ & 6.690890 & 0.728677 & -1.470570 \\
\hline $\mathrm{C}$ & 6.108111 & 1.670027 & -2.539970 \\
\hline $\mathrm{C}$ & 7.940200 & -0.001914 & -2.001030 \\
\hline $\mathrm{H}$ & 7.010381 & 1.351757 & -0.623840 \\
\hline $\mathrm{H}$ & 5.237091 & 2.212368 & -2.158060 \\
\hline $\mathrm{H}$ & 6.858731 & 2.404627 & -2.855330 \\
\hline $\mathrm{H}$ & 5.792601 & 1.117147 & -3.432780 \\
\hline $\mathrm{H}$ & 8.383950 & -0.639684 & -1.228630 \\
\hline $\mathrm{H}$ & 7.690640 & -0.639914 & -2.857300 \\
\hline $\mathrm{H}$ & 8.700310 & 0.717256 & -2.329660 \\
\hline $\mathrm{C}$ & 4.294090 & -1.275202 & 2.491880 \\
\hline $\mathrm{C}$ & 3.787500 & 0.075188 & 3.030860 \\
\hline $\mathrm{C}$ & 5.582179 & -1.709233 & 3.220010 \\
\hline $\mathrm{H}$ & 3.523339 & -2.013212 & 2.728180 \\
\hline $\mathrm{H}$ & 2.830740 & 0.337939 & 2.570990 \\
\hline $\mathrm{H}$ & 3.641150 & 0.016348 & 4.116610 \\
\hline $\mathrm{H}$ & 4.502951 & 0.884098 & 2.839930 \\
\hline $\mathrm{H}$ & 5.928799 & -2.691163 & 2.877770 \\
\hline $\mathrm{H}$ & 6.397540 & -0.995613 & 3.051980 \\
\hline $\mathrm{H}$ & 5.409659 & -1.768742 & 4.301510 \\
\hline $\mathrm{C}$ & 3.401769 & -3.027962 & -2.293390 \\
\hline $\mathrm{C}$ & 4.443638 & -3.964372 & -2.938020 \\
\hline $\mathrm{C}$ & 2.591119 & -2.276501 & -3.365920 \\
\hline $\mathrm{H}$ & 2.699518 & -3.664771 & -1.748910 \\
\hline $\mathrm{H}$ & 4.992418 & -4.531092 & -2.177330 \\
\hline $\mathrm{H}$ & 3.952468 & -4.680822 & -3.607550 \\
\hline $\mathrm{H}$ & 5.177279 & -3.404192 & -3.529630 \\
\hline $\mathrm{H}$ & 1.824139 & -1.646491 & -2.905850 \\
\hline $\mathrm{H}$ & 3.233109 & -1.639461 & -3.986090 \\
\hline $\mathrm{H}$ & 2.091469 & -2.989451 & -4.033110 \\
\hline $\mathrm{C}$ & 0.392422 & 2.877010 & 3.661170 \\
\hline $\mathrm{C}$ & 0.338611 & 1.691320 & 3.896150 \\
\hline $\mathrm{H}$ & 0.295540 & 0.635080 & 4.036780 \\
\hline
\end{tabular}

\section{1d allylboration TS 5 (TS-3Si-B)}

B3LYP Energy =

B3LYP Free Energy =

M06-2X Derived Solution Free Energy $(\mathrm{DCM})=$ M06-2X Derived Solution Free Energy (toluene) $=$ Number of Imaginary Frequencies $=$

$-3456.176276$

$-3454.948750$

$-3454.469768$

$-3454.461412$

$1(-327.61)$

B3LYP/6-31G(d) Geometry

$\begin{array}{lll}\mathrm{O} & -2.919419 & -2.737101 \\ \mathrm{~B} & -2.375959 & -1.658931 \\ \mathrm{O} & -2.795729 & -1.746181 \\ \mathrm{O} & -0.912289 & -1.725630 \\ \mathrm{C} & -2.392969 & -0.876161\end{array}$

$-1.765200$

$-1.007300$

0.431600

$-1.147270$

1.311090 


\begin{tabular}{|c|c|c|c|}
\hline $\mathrm{C}$ & -0.587909 & -2.850700 & -2.016730 \\
\hline $\mathrm{C}$ & -2.915069 & -0.948041 & 2.692040 \\
\hline $\mathrm{C}$ & -4.186489 & -1.481852 & 3.039820 \\
\hline $\mathrm{C}$ & -2.071520 & -0.460971 & 3.702310 \\
\hline $\mathrm{C}$ & -2.452150 & -0.498951 & 5.040590 \\
\hline $\mathrm{C}$ & -4.552739 & -1.507232 & 4.400850 \\
\hline $\mathrm{C}$ & -3.699219 & -1.025292 & 5.387990 \\
\hline $\mathrm{C}$ & -1.894668 & -3.726931 & -1.935750 \\
\hline $\mathrm{C}$ & -1.913788 & -4.673831 & -0.721940 \\
\hline $\mathrm{C}$ & -2.202658 & -4.524271 & -3.205170 \\
\hline $\mathrm{C}$ & 0.666682 & -3.532180 & -1.475360 \\
\hline $\mathrm{C}$ & -0.334479 & -2.288950 & -3.423610 \\
\hline $\mathrm{C}$ & -3.079810 & -0.166101 & -1.547500 \\
\hline $\mathrm{C}$ & -2.737710 & 0.837939 & -0.577390 \\
\hline $\mathrm{C}$ & -3.336530 & 0.909189 & 0.664530 \\
\hline $\mathrm{H}$ & -1.372880 & -0.498791 & 1.231410 \\
\hline $\mathrm{H}$ & -1.780120 & -0.123711 & 5.806810 \\
\hline $\mathrm{H}$ & -5.523169 & -1.915172 & 4.665730 \\
\hline $\mathrm{H}$ & -4.007739 & -1.060902 & 6.429240 \\
\hline $\mathrm{H}$ & -1.689088 & -4.142951 & 0.206690 \\
\hline $\mathrm{H}$ & -2.917668 & -5.099781 & -0.627500 \\
\hline $\mathrm{H}$ & -1.200418 & -5.497371 & -0.837520 \\
\hline $\mathrm{H}$ & -3.127778 & -5.092721 & -3.062080 \\
\hline $\mathrm{H}$ & -2.342098 & -3.868241 & -4.067450 \\
\hline $\mathrm{H}$ & -1.401138 & -5.237551 & -3.430660 \\
\hline $\mathrm{H}$ & 1.526091 & -2.860070 & -1.534130 \\
\hline $\mathrm{H}$ & 0.541732 & -3.821460 & -0.429630 \\
\hline $\mathrm{H}$ & 0.903632 & -4.429600 & -2.058720 \\
\hline $\mathrm{H}$ & -1.240019 & -1.827961 & -3.829320 \\
\hline $\mathrm{H}$ & 0.446751 & -1.523940 & -3.376620 \\
\hline $\mathrm{H}$ & -0.002599 & -3.069070 & -4.117120 \\
\hline $\mathrm{H}$ & -2.664900 & 0.003549 & -2.543030 \\
\hline $\mathrm{H}$ & -4.146410 & -0.400052 & -1.596540 \\
\hline $\mathrm{H}$ & -1.822230 & 1.410029 & -0.723230 \\
\hline $\mathrm{H}$ & -4.334900 & 0.502888 & 0.805720 \\
\hline $\mathrm{H}$ & -3.010950 & 1.654949 & 1.380990 \\
\hline $\mathrm{C}$ & 4.687131 & -1.616668 & 0.222130 \\
\hline $\mathrm{C}$ & 4.422281 & -2.149318 & 1.505450 \\
\hline $\mathrm{C}$ & 4.190722 & -3.524058 & 1.630220 \\
\hline $\mathrm{C}$ & 4.223012 & -4.393828 & 0.539740 \\
\hline $\mathrm{C}$ & 4.484982 & -3.844568 & -0.718270 \\
\hline $\mathrm{C}$ & 4.721221 & -2.477908 & -0.905120 \\
\hline $\mathrm{O}$ & 2.856410 & 0.532831 & 0.738530 \\
\hline$P$ & 1.399930 & 0.879740 & 0.118720 \\
\hline $\mathrm{C}$ & 4.106580 & 0.877321 & 0.231760 \\
\hline $\mathrm{O}$ & 0.379530 & 0.880500 & 1.194340 \\
\hline $\mathrm{O}$ & 1.658679 & 2.277801 & -0.664090 \\
\hline $\mathrm{C}$ & 4.461089 & 2.231212 & 0.069550 \\
\hline $\mathrm{C}$ & 5.032200 & -0.166608 & 0.050710 \\
\hline $\mathrm{C}$ & 2.193049 & 3.384751 & -0.002970 \\
\hline $\mathrm{C}$ & 3.552729 & 3.372161 & 0.352450 \\
\hline $\mathrm{C}$ & 6.342720 & 0.179512 & -0.308630 \\
\hline $\mathrm{C}$ & 5.776319 & 2.513942 & -0.340790 \\
\hline
\end{tabular}




\begin{tabular}{|c|c|c|c|}
\hline $\mathrm{C}$ & 6.713060 & 1.504423 & -0.519470 \\
\hline $\mathrm{C}$ & 1.375028 & 4.514120 & 0.164890 \\
\hline $\mathrm{C}$ & 4.077418 & 4.533361 & 0.947150 \\
\hline $\mathrm{C}$ & 1.962118 & 5.659791 & 0.720690 \\
\hline $\mathrm{C}$ & 3.295248 & 5.668041 & 1.124090 \\
\hline $\mathrm{C}$ & -0.075382 & 4.540110 & -0.216750 \\
\hline $\mathrm{C}$ & -1.060442 & 4.622179 & 0.797150 \\
\hline $\mathrm{C}$ & -2.405832 & 4.722279 & 0.421120 \\
\hline $\mathrm{C}$ & -2.812862 & 4.750459 & -0.913440 \\
\hline $\mathrm{C}$ & -1.820952 & 4.666229 & -1.894610 \\
\hline $\mathrm{C}$ & -0.462312 & 4.561960 & -1.578920 \\
\hline $\mathrm{O}$ & 1.138090 & -0.074060 & -1.110720 \\
\hline $\mathrm{H}$ & 3.998122 & -3.936939 & 2.618220 \\
\hline $\mathrm{H}$ & 4.519532 & -4.501198 & -1.583990 \\
\hline $\mathrm{H}$ & 7.070690 & -0.616887 & -0.431770 \\
\hline $\mathrm{H}$ & 6.054899 & 3.547162 & -0.520870 \\
\hline $\mathrm{H}$ & 7.727069 & 1.748533 & -0.822800 \\
\hline $\mathrm{H}$ & 5.111918 & 4.532942 & 1.274880 \\
\hline $\mathrm{H}$ & 1.349578 & 6.548050 & 0.843510 \\
\hline $\mathrm{H}$ & 3.723188 & 6.558261 & 1.576130 \\
\hline $\mathrm{H}$ & -3.165462 & 4.787029 & 1.197380 \\
\hline $\mathrm{H}$ & -2.110422 & 4.697709 & -2.941980 \\
\hline $\mathrm{H}$ & 0.310740 & -0.665710 & -1.042840 \\
\hline $\mathrm{C}$ & 4.454161 & -1.300658 & 2.776500 \\
\hline $\mathrm{C}$ & 5.632501 & -1.710478 & 3.683410 \\
\hline $\mathrm{C}$ & 3.121321 & -1.346389 & 3.547020 \\
\hline $\mathrm{H}$ & 4.623820 & -0.259758 & 2.490130 \\
\hline $\mathrm{H}$ & 6.587011 & -1.642608 & 3.149280 \\
\hline $\mathrm{H}$ & 5.686491 & -1.054608 & 4.560910 \\
\hline $\mathrm{H}$ & 5.524631 & -2.740398 & 4.043450 \\
\hline $\mathrm{H}$ & 2.293681 & -0.994599 & 2.924290 \\
\hline $\mathrm{H}$ & 2.886811 & -2.361009 & 3.890750 \\
\hline $\mathrm{H}$ & 3.175810 & -0.702869 & 4.433620 \\
\hline $\mathrm{C}$ & 5.025051 & -1.982378 & -2.322850 \\
\hline $\mathrm{C}$ & 3.832511 & -2.174159 & -3.277500 \\
\hline $\mathrm{C}$ & 6.297131 & -2.638848 & -2.895040 \\
\hline $\mathrm{H}$ & 5.214151 & -0.907398 & -2.276960 \\
\hline $\mathrm{H}$ & 2.958361 & -1.620109 & -2.922170 \\
\hline $\mathrm{H}$ & 4.084501 & -1.803119 & -4.278580 \\
\hline $\mathrm{H}$ & 3.552361 & -3.229589 & -3.374040 \\
\hline $\mathrm{H}$ & 7.160581 & -2.481287 & -2.238590 \\
\hline $\mathrm{H}$ & 6.173242 & -3.720598 & -3.022860 \\
\hline $\mathrm{H}$ & 6.535991 & -2.214848 & -3.877790 \\
\hline $\mathrm{C}$ & 4.038562 & -5.893189 & 0.740330 \\
\hline $\mathrm{C}$ & 5.361723 & -6.650578 & 0.510850 \\
\hline $\mathrm{C}$ & 2.912893 & -6.482589 & -0.127640 \\
\hline $\mathrm{H}$ & 3.754233 & -6.042979 & 1.790980 \\
\hline $\mathrm{H}$ & 6.155803 & -6.265288 & 1.159760 \\
\hline $\mathrm{H}$ & 5.238123 & -7.720338 & 0.719050 \\
\hline $\mathrm{H}$ & 5.700423 & -6.548288 & -0.527210 \\
\hline $\mathrm{H}$ & 1.960183 & -5.976149 & 0.058690 \\
\hline $\mathrm{H}$ & 3.138993 & -6.388989 & -1.196520 \\
\hline $\mathrm{H}$ & 2.780663 & -7.549479 & 0.088730 \\
\hline
\end{tabular}




\begin{tabular}{|c|c|c|c|}
\hline $\mathrm{C}$ & -4.284272 & 4.916788 & -1.272420 \\
\hline $\mathrm{C}$ & -4.787961 & 3.840188 & -2.249860 \\
\hline $\mathrm{C}$ & -4.564692 & 6.330838 & -1.819210 \\
\hline $\mathrm{H}$ & -4.855872 & 4.807158 & -0.340490 \\
\hline $\mathrm{H}$ & -4.610581 & 2.833448 & -1.857800 \\
\hline $\mathrm{H}$ & -5.864441 & 3.955118 & -2.423950 \\
\hline $\mathrm{H}$ & -4.288621 & 3.914158 & -3.223240 \\
\hline $\mathrm{H}$ & -4.249383 & 7.101158 & -1.106870 \\
\hline $\mathrm{H}$ & -4.025532 & 6.504538 & -2.758330 \\
\hline $\mathrm{H}$ & -5.635042 & 6.465288 & -2.017360 \\
\hline $\mathrm{C}$ & -0.719922 & 4.614320 & 2.289180 \\
\hline $\mathrm{C}$ & -1.362241 & 3.424469 & 3.025510 \\
\hline $\mathrm{C}$ & -1.096412 & 5.948739 & 2.962850 \\
\hline $\mathrm{H}$ & 0.361348 & 4.493530 & 2.391050 \\
\hline $\mathrm{H}$ & -1.033231 & 2.479709 & 2.583300 \\
\hline $\mathrm{H}$ & -1.066861 & 3.429719 & 4.082060 \\
\hline $\mathrm{H}$ & -2.457811 & 3.468269 & 2.992580 \\
\hline $\mathrm{H}$ & -0.605532 & 6.799060 & 2.475640 \\
\hline $\mathrm{H}$ & -2.177672 & 6.126869 & 2.926220 \\
\hline $\mathrm{H}$ & -0.795992 & 5.942620 & 4.017590 \\
\hline $\mathrm{C}$ & 0.551458 & 4.531810 & -2.722620 \\
\hline $\mathrm{C}$ & 0.537048 & 5.854080 & -3.515730 \\
\hline $\mathrm{C}$ & 0.340169 & 3.323840 & -3.655120 \\
\hline $\mathrm{H}$ & 1.551138 & 4.432500 & -2.291280 \\
\hline $\mathrm{H}$ & 0.729188 & 6.711980 & -2.861560 \\
\hline $\mathrm{H}$ & 1.308358 & 5.841280 & -4.295280 \\
\hline $\mathrm{H}$ & -0.429572 & 6.018880 & -4.006120 \\
\hline $\mathrm{H}$ & 0.406209 & 2.383330 & -3.099960 \\
\hline $\mathrm{H}$ & -0.636461 & 3.362480 & -4.152540 \\
\hline $\mathrm{H}$ & 1.108269 & 3.310240 & -4.437910 \\
\hline $\mathrm{C}$ & -5.108539 & -1.972832 & 2.074690 \\
\hline $\mathrm{C}$ & -5.931309 & -2.404482 & 1.289170 \\
\hline $\mathrm{H}$ & -1.103020 & -0.056561 & 3.419270 \\
\hline $\mathrm{C}$ & -6.830939 & -2.918773 & 0.311290 \\
\hline $\mathrm{C}$ & -8.194619 & -3.104723 & 0.613630 \\
\hline $\mathrm{C}$ & -6.358239 & -3.248263 & -0.977010 \\
\hline $\mathrm{C}$ & -9.065818 & -3.604644 & -0.350190 \\
\hline $\mathrm{H}$ & -8.556729 & -2.851843 & 1.605710 \\
\hline $\mathrm{C}$ & -7.241058 & -3.747183 & -1.931630 \\
\hline $\mathrm{H}$ & -5.304909 & -3.116582 & -1.210770 \\
\hline $\mathrm{C}$ & -8.592798 & -3.926433 & -1.625020 \\
\hline $\mathrm{H}$ & -10.115908 & -3.743474 & -0.107000 \\
\hline $\mathrm{H}$ & -6.870198 & -3.998273 & -2.921870 \\
\hline $\mathrm{H}$ & -9.275148 & -4.316224 & -2.375780 \\
\hline
\end{tabular}

\section{1d allylboration TS 6}

B3LYP Energy =

B3LYP Free Energy =

M06-2X Derived Solution Free Energy $(\mathrm{DCM})=$

M06-2X Derived Solution Free Energy (toluene) $=$ Number of Imaginary Frequencies $=$

$-3456.174679$

$-3454.946898$

$-3454.468851$

$-3454.460247$

$1(-270.34)$

B3LYP/6-31G(d) Geometry 


\begin{tabular}{|c|c|c|c|}
\hline $\mathrm{O}$ & -0.007752 & 4.313460 & -2.845240 \\
\hline B & 0.324088 & 3.421890 & -1.789190 \\
\hline $\mathrm{O}$ & 0.224868 & 4.156870 & -0.458540 \\
\hline $\mathrm{O}$ & -0.623941 & 2.298999 & -1.859830 \\
\hline $\mathrm{C}$ & 0.570338 & 3.713150 & 0.707450 \\
\hline $\mathrm{C}$ & -1.532561 & 2.536649 & -2.980430 \\
\hline $\mathrm{C}$ & 0.662958 & 4.720200 & 1.787170 \\
\hline $\mathrm{C}$ & 0.631847 & 6.082180 & 1.446250 \\
\hline $\mathrm{C}$ & 0.753908 & 4.347910 & 3.154470 \\
\hline $\mathrm{C}$ & 0.807708 & 5.367180 & 4.129620 \\
\hline $\mathrm{C}$ & 0.688887 & 7.069920 & 2.421940 \\
\hline $\mathrm{C}$ & 0.777007 & 6.708710 & 3.770860 \\
\hline $\mathrm{C}$ & -1.369692 & 4.083879 & -3.222570 \\
\hline $\mathrm{C}$ & -2.284932 & 4.937609 & -2.324500 \\
\hline $\mathrm{C}$ & -1.544912 & 4.522159 & -4.678880 \\
\hline $\mathrm{C}$ & -2.934591 & 2.099368 & -2.561200 \\
\hline $\mathrm{C}$ & -1.038641 & 1.699669 & -4.170190 \\
\hline $\mathrm{C}$ & 1.988659 & 2.999201 & -1.895430 \\
\hline $\mathrm{C}$ & 2.430349 & 2.485421 & -0.620970 \\
\hline $\mathrm{C}$ & 2.704478 & 3.296311 & 0.456100 \\
\hline $\mathrm{H}$ & 0.329839 & 2.685440 & 0.984270 \\
\hline $\mathrm{H}$ & 0.549837 & 6.345220 & 0.397330 \\
\hline $\mathrm{H}$ & 0.873998 & 5.078240 & 5.173610 \\
\hline $\mathrm{H}$ & 0.659966 & 8.117310 & 2.135470 \\
\hline $\mathrm{H}$ & 0.819217 & 7.473290 & 4.541680 \\
\hline $\mathrm{H}$ & -2.207152 & 4.646019 & -1.273810 \\
\hline $\mathrm{H}$ & -1.971123 & 5.983289 & -2.406200 \\
\hline $\mathrm{H}$ & -3.335142 & 4.869398 & -2.629390 \\
\hline $\mathrm{H}$ & -1.405923 & 5.606059 & -4.749950 \\
\hline $\mathrm{H}$ & -0.808382 & 4.047569 & -5.331050 \\
\hline $\mathrm{H}$ & -2.549262 & 4.284919 & -5.049390 \\
\hline $\mathrm{H}$ & -2.961571 & 1.022598 & -2.377240 \\
\hline $\mathrm{H}$ & -3.249101 & 2.601818 & -1.644050 \\
\hline $\mathrm{H}$ & -3.662711 & 2.324178 & -3.349510 \\
\hline $\mathrm{H}$ & -0.043191 & 2.022760 & -4.489240 \\
\hline $\mathrm{H}$ & -0.981740 & 0.645059 & -3.885180 \\
\hline $\mathrm{H}$ & -1.718451 & 1.781409 & -5.024950 \\
\hline $\mathrm{H}$ & 2.042839 & 2.272711 & -2.709920 \\
\hline $\mathrm{H}$ & 2.463568 & 3.941531 & -2.183040 \\
\hline $\mathrm{H}$ & 2.320039 & 1.419841 & -0.428760 \\
\hline $\mathrm{H}$ & 2.952478 & 4.343211 & 0.303170 \\
\hline $\mathrm{H}$ & 2.980699 & 2.869501 & 1.414850 \\
\hline $\mathrm{C}$ & -3.967209 & -1.958972 & 0.096050 \\
\hline $\mathrm{C}$ & -4.279560 & -1.188282 & 1.238680 \\
\hline $\mathrm{C}$ & -5.238400 & -0.174363 & 1.126430 \\
\hline $\mathrm{C}$ & -5.903070 & 0.101747 & -0.068610 \\
\hline $\mathrm{C}$ & -5.570330 & -0.666913 & -1.187440 \\
\hline $\mathrm{C}$ & -4.620569 & -1.692932 & -1.134860 \\
\hline $\mathrm{O}$ & -1.140449 & -1.725381 & 0.607580 \\
\hline $\mathrm{P}$ & 0.011500 & -0.920260 & -0.202040 \\
\hline $\mathrm{C}$ & -1.636259 & -2.998801 & 0.338440 \\
\hline $\mathrm{O}$ & 0.723100 & 0.004730 & 0.713900 \\
\hline $\mathrm{O}$ & 0.884441 & -2.114720 & -0.870500 \\
\hline
\end{tabular}




\begin{tabular}{|c|c|c|c|}
\hline $\mathrm{C}$ & -0.779718 & -4.117031 & 0.374120 \\
\hline $\mathrm{C}$ & -3.028719 & -3.126932 & 0.185760 \\
\hline $\mathrm{C}$ & 1.495461 & -3.070720 & -0.057020 \\
\hline $\mathrm{C}$ & 0.691122 & -4.038520 & 0.569250 \\
\hline $\mathrm{C}$ & -3.557628 & -4.422322 & 0.092690 \\
\hline $\mathrm{C}$ & -1.362628 & -5.388771 & 0.230610 \\
\hline $\mathrm{C}$ & -2.736828 & -5.546182 & 0.106000 \\
\hline $\mathrm{C}$ & 2.899061 & -3.092569 & 0.001560 \\
\hline $\mathrm{C}$ & 1.340532 & -5.022500 & 1.336320 \\
\hline $\mathrm{C}$ & 3.494942 & -4.125039 & 0.740140 \\
\hline $\mathrm{C}$ & 2.727342 & -5.069059 & 1.418770 \\
\hline $\mathrm{C}$ & 3.758611 & -2.071369 & -0.683230 \\
\hline $\mathrm{C}$ & 4.534260 & -1.176188 & 0.093220 \\
\hline $\mathrm{C}$ & 5.370700 & -0.265338 & -0.565070 \\
\hline $\mathrm{C}$ & 5.473530 & -0.208408 & -1.954640 \\
\hline $\mathrm{C}$ & 4.702090 & -1.106188 & -2.697920 \\
\hline $\mathrm{C}$ & 3.850541 & -2.037839 & -2.097200 \\
\hline $\mathrm{O}$ & -0.639420 & -0.282311 & -1.484910 \\
\hline $\mathrm{H}$ & -5.490210 & 0.410847 & 2.008270 \\
\hline $\mathrm{H}$ & -6.072940 & -0.472453 & -2.131860 \\
\hline $\mathrm{H}$ & -4.633448 & -4.533392 & -0.005380 \\
\hline $\mathrm{H}$ & -0.714997 & -6.259211 & 0.208430 \\
\hline $\mathrm{H}$ & -3.165737 & -6.539432 & 0.009480 \\
\hline $\mathrm{H}$ & 0.740542 & -5.755880 & 1.865750 \\
\hline $\mathrm{H}$ & 4.578802 & -4.170878 & 0.784450 \\
\hline $\mathrm{H}$ & 3.211053 & -5.844039 & 2.006500 \\
\hline $\mathrm{H}$ & 5.967620 & 0.422722 & 0.029340 \\
\hline $\mathrm{H}$ & 4.773960 & -1.091418 & -3.782580 \\
\hline $\mathrm{H}$ & -0.565440 & 0.738469 & -1.542620 \\
\hline $\mathrm{C}$ & -3.659949 & -1.459292 & 2.608540 \\
\hline $\mathrm{C}$ & -4.712029 & -2.005352 & 3.594720 \\
\hline $\mathrm{C}$ & -2.953150 & -0.218862 & 3.184540 \\
\hline $\mathrm{H}$ & -2.899459 & -2.235912 & 2.491950 \\
\hline $\mathrm{H}$ & -5.182759 & -2.917183 & 3.209860 \\
\hline $\mathrm{H}$ & -4.246419 & -2.243122 & 4.558840 \\
\hline $\mathrm{H}$ & -5.506450 & -1.272533 & 3.779140 \\
\hline $\mathrm{H}$ & -2.185530 & 0.151319 & 2.498570 \\
\hline $\mathrm{H}$ & -3.659600 & 0.596948 & 3.378830 \\
\hline $\mathrm{H}$ & -2.464410 & -0.466691 & 4.133650 \\
\hline $\mathrm{C}$ & -4.347459 & -2.494452 & -2.411490 \\
\hline $\mathrm{C}$ & -3.782349 & -1.616842 & -3.543700 \\
\hline $\mathrm{C}$ & -5.603599 & -3.254083 & -2.882350 \\
\hline $\mathrm{H}$ & -3.583599 & -3.243392 & -2.188920 \\
\hline $\mathrm{H}$ & -2.839190 & -1.151862 & -3.241210 \\
\hline $\mathrm{H}$ & -3.589559 & -2.226342 & -4.435130 \\
\hline $\mathrm{H}$ & -4.479360 & -0.820602 & -3.830340 \\
\hline $\mathrm{H}$ & -5.990688 & -3.915103 & -2.098580 \\
\hline $\mathrm{H}$ & -6.410099 & -2.565623 & -3.160570 \\
\hline $\mathrm{H}$ & -5.373118 & -3.868513 & -3.761060 \\
\hline $\mathrm{C}$ & -6.982171 & 1.175917 & -0.126170 \\
\hline $\mathrm{C}$ & -8.372320 & 0.562146 & -0.383530 \\
\hline $\mathrm{C}$ & -6.669381 & 2.275687 & -1.156750 \\
\hline $\mathrm{H}$ & -7.013001 & 1.652907 & 0.863090 \\
\hline
\end{tabular}




\begin{tabular}{|c|c|c|c|}
\hline $\mathrm{H}$ & -8.618310 & -0.191824 & 0.372200 \\
\hline $\mathrm{H}$ & -9.148721 & 1.336506 & -0.359920 \\
\hline $\mathrm{H}$ & -8.415080 & 0.076236 & -1.365710 \\
\hline $\mathrm{H}$ & -5.701431 & 2.745927 & -0.954430 \\
\hline $\mathrm{H}$ & -6.638221 & 1.871777 & -2.175680 \\
\hline $\mathrm{H}$ & -7.440101 & 3.055496 & -1.132970 \\
\hline $\mathrm{C}$ & 6.409480 & 0.787423 & -2.627430 \\
\hline $\mathrm{C}$ & 5.649729 & 1.772902 & -3.534820 \\
\hline $\mathrm{C}$ & 7.534970 & 0.078193 & -3.404700 \\
\hline $\mathrm{H}$ & 6.882689 & 1.374673 & -1.828410 \\
\hline $\mathrm{H}$ & 4.876219 & 2.310292 & -2.976210 \\
\hline $\mathrm{H}$ & 6.337349 & 2.511382 & -3.964540 \\
\hline $\mathrm{H}$ & 5.160139 & 1.252072 & -4.366340 \\
\hline $\mathrm{H}$ & 8.098350 & -0.601137 & -2.755620 \\
\hline $\mathrm{H}$ & 7.132740 & -0.511847 & -4.236890 \\
\hline $\mathrm{H}$ & 8.235500 & 0.810183 & -3.824410 \\
\hline $\mathrm{C}$ & 4.514740 & -1.161858 & 1.623480 \\
\hline $\mathrm{C}$ & 4.084370 & 0.203151 & 2.190070 \\
\hline $\mathrm{C}$ & 5.876301 & -1.590878 & 2.206010 \\
\hline $\mathrm{H}$ & 3.771341 & -1.886249 & 1.965430 \\
\hline $\mathrm{H}$ & 3.084520 & 0.467241 & 1.834770 \\
\hline $\mathrm{H}$ & 4.054030 & 0.164431 & 3.285110 \\
\hline $\mathrm{H}$ & 4.780009 & 1.001102 & 1.904200 \\
\hline $\mathrm{H}$ & 6.175801 & -2.582628 & 1.847900 \\
\hline $\mathrm{H}$ & 6.669980 & -0.887467 & 1.927580 \\
\hline $\mathrm{H}$ & 5.830071 & -1.624998 & 3.301120 \\
\hline $\mathrm{C}$ & 3.100801 & -3.020319 & -2.997360 \\
\hline $\mathrm{C}$ & 4.079862 & -3.968449 & -3.718990 \\
\hline $\mathrm{C}$ & 2.179071 & -2.305449 & -4.003080 \\
\hline $\mathrm{H}$ & 2.463812 & -3.646179 & -2.366870 \\
\hline $\mathrm{H}$ & 4.706902 & -4.511968 & -3.003210 \\
\hline $\mathrm{H}$ & 3.529402 & -4.704729 & -4.317220 \\
\hline $\mathrm{H}$ & 4.745921 & -3.420278 & -4.395880 \\
\hline $\mathrm{H}$ & 1.459621 & -1.662810 & -3.487480 \\
\hline $\mathrm{H}$ & 2.748991 & -1.686919 & -4.706660 \\
\hline $\mathrm{H}$ & 1.618451 & -3.041400 & -4.592300 \\
\hline $\mathrm{C}$ & 0.801339 & 2.997180 & 3.600260 \\
\hline $\mathrm{C}$ & 0.851839 & 1.891030 & 4.107020 \\
\hline $\mathrm{C}$ & 0.914900 & 0.613810 & 4.736990 \\
\hline $\mathrm{C}$ & 1.073260 & 0.528840 & 6.135760 \\
\hline $\mathrm{C}$ & 0.821870 & -0.571730 & 3.980250 \\
\hline $\mathrm{C}$ & 1.137740 & -0.713370 & 6.760040 \\
\hline $\mathrm{H}$ & 1.144109 & 1.442540 & 6.718270 \\
\hline $\mathrm{C}$ & 0.889171 & -1.808880 & 4.617540 \\
\hline $\mathrm{H}$ & 0.701490 & -0.503030 & 2.904080 \\
\hline $\mathrm{C}$ & 1.046861 & -1.885320 & 6.003830 \\
\hline $\mathrm{H}$ & 1.259560 & -0.768030 & 7.838570 \\
\hline $\mathrm{H}$ & 0.819751 & -2.717340 & 4.025000 \\
\hline $\mathrm{H}$ & 1.098821 & -2.853760 & 6.494440 \\
\hline
\end{tabular}

1d propargylboration TS 5

B3LYP Energy =

$-3454.925782$

B3LYP Free Energy =

$-3453.722619$ 
M06-2X Derived Solution Free Energy (toluene) = Number of Imaginary Frequencies $=$

B3LYP/6-31G(d) Geometry

\begin{tabular}{|c|c|c|c|}
\hline $\mathrm{O}$ & -2.649511 & -3.016469 & -1.715120 \\
\hline B & -2.107231 & -1.956079 & -0.936100 \\
\hline $\mathrm{O}$ & -2.556911 & -2.018409 & 0.478180 \\
\hline $\mathrm{O}$ & -0.644781 & -2.018160 & -1.064960 \\
\hline $\mathrm{C}$ & -2.217411 & -1.081919 & 1.333920 \\
\hline $\mathrm{C}$ & -0.315441 & -3.113640 & -1.966980 \\
\hline $\mathrm{C}$ & -2.742991 & -1.177639 & 2.718410 \\
\hline $\mathrm{C}$ & -4.009371 & -1.729809 & 3.051730 \\
\hline $\mathrm{C}$ & -2.304730 & -0.736989 & 5.072570 \\
\hline $\mathrm{C}$ & -4.384651 & -1.772389 & 4.410010 \\
\hline $\mathrm{C}$ & -3.545101 & -1.286109 & 5.407120 \\
\hline $\mathrm{C}$ & -1.617421 & -3.999269 & -1.904000 \\
\hline $\mathrm{C}$ & -1.634442 & -4.962869 & -0.704050 \\
\hline $\mathrm{C}$ & -1.921602 & -4.776349 & -3.186140 \\
\hline $\mathrm{C}$ & 0.946539 & -3.797140 & -1.444990 \\
\hline $\mathrm{C}$ & -0.071821 & -2.516400 & -3.360770 \\
\hline $\mathrm{C}$ & -2.688520 & -0.432789 & -1.529830 \\
\hline $\mathrm{C}$ & -2.891050 & 0.366371 & -0.562270 \\
\hline $\mathrm{C}$ & -3.001280 & 0.689721 & 0.757410 \\
\hline $\mathrm{H}$ & -1.194680 & -0.706120 & 1.268300 \\
\hline $\mathrm{H}$ & -1.644230 & -0.356189 & 5.846070 \\
\hline $\mathrm{H}$ & -5.351141 & -2.196028 & 4.664510 \\
\hline $\mathrm{H}$ & -3.860141 & -1.334929 & 6.445900 \\
\hline $\mathrm{H}$ & -1.412061 & -4.442970 & 0.231530 \\
\hline $\mathrm{H}$ & -2.637172 & -5.392669 & -0.616180 \\
\hline $\mathrm{H}$ & -0.918182 & -5.782280 & -0.830350 \\
\hline $\mathrm{H}$ & -2.845502 & -5.349129 & -3.053560 \\
\hline $\mathrm{H}$ & -2.060791 & -4.107649 & -4.038720 \\
\hline $\mathrm{H}$ & -1.117762 & -5.483930 & -3.421030 \\
\hline $\mathrm{H}$ & 1.795149 & -3.109040 & -1.479060 \\
\hline $\mathrm{H}$ & 0.821679 & -4.121740 & -0.409840 \\
\hline $\mathrm{H}$ & 1.197419 & -4.671880 & -2.056790 \\
\hline $\mathrm{H}$ & -0.983101 & -2.056390 & -3.754740 \\
\hline $\mathrm{H}$ & 0.700279 & -1.743760 & -3.299940 \\
\hline $\mathrm{H}$ & 0.265459 & -3.277430 & -4.072520 \\
\hline $\mathrm{H}$ & -2.784370 & -0.310929 & -2.599480 \\
\hline $\mathrm{H}$ & -3.999240 & 0.721581 & 1.193450 \\
\hline $\mathrm{H}$ & -2.272560 & 1.365721 & 1.195860 \\
\hline $\mathrm{C}$ & 4.912069 & -1.102291 & 0.188340 \\
\hline $\mathrm{C}$ & 4.769729 & -1.540781 & 1.525640 \\
\hline $\mathrm{C}$ & 4.701579 & -2.915181 & 1.781250 \\
\hline $\mathrm{C}$ & 4.777119 & -3.872411 & 0.769430 \\
\hline $\mathrm{C}$ & 4.919899 & -3.415831 & -0.543650 \\
\hline $\mathrm{C}$ & 4.995399 & -2.054961 & -0.860060 \\
\hline $\mathrm{O}$ & 2.857410 & 0.847369 & 0.639180 \\
\hline $\mathrm{P}$ & 1.342780 & 0.933540 & 0.069070 \\
\hline $\mathrm{C}$ & 4.023750 & 1.290899 & 0.022780 \\
\hline $\mathrm{O}$ & 0.380510 & 0.840170 & 1.193690 \\
\hline $\mathrm{O}$ & 1.353170 & 2.313120 & -0.785820 \\
\hline
\end{tabular}

$-3453.240432$

$1(-343.45)$

1.715120

6100

064960

333920

410010

904000

$-3.186140$

$-3.360770$

0.562270

.268300

664510

445900

0.830350

$-3.053560$

4.038720

0.409840

$-3.299940$

1.193450

1.195860

1.781250

543650

860060

0.069070

193690 


\begin{tabular}{|c|c|c|c|}
\hline $\mathrm{C}$ & 4.191991 & 2.657299 & -0.274490 \\
\hline $\mathrm{C}$ & 5.063780 & 0.355789 & -0.129780 \\
\hline $\mathrm{C}$ & 1.792431 & 3.517480 & -0.233890 \\
\hline $\mathrm{C}$ & 3.167911 & 3.698739 & -0.003190 \\
\hline $\mathrm{C}$ & 6.296190 & 0.825478 & -0.606040 \\
\hline $\mathrm{C}$ & 5.432931 & 3.059759 & -0.800090 \\
\hline $\mathrm{C}$ & 6.479170 & 2.159998 & -0.956950 \\
\hline $\mathrm{C}$ & 0.854121 & 4.548260 & -0.055180 \\
\hline $\mathrm{C}$ & 3.588031 & 4.956549 & 0.464480 \\
\hline $\mathrm{C}$ & 1.336241 & 5.796160 & 0.366170 \\
\hline $\mathrm{C}$ & 2.686551 & 5.999249 & 0.637890 \\
\hline $\mathrm{C}$ & -0.619419 & 4.379310 & -0.280870 \\
\hline $\mathrm{C}$ & -1.502689 & 4.440971 & 0.825380 \\
\hline $\mathrm{C}$ & -2.881559 & 4.372691 & 0.588800 \\
\hline $\mathrm{C}$ & -3.419979 & 4.255621 & -0.693550 \\
\hline $\mathrm{C}$ & -2.526769 & 4.194491 & -1.766330 \\
\hline $\mathrm{C}$ & -1.140409 & 4.252670 & -1.591070 \\
\hline $\mathrm{O}$ & 1.198260 & -0.118290 & -1.097790 \\
\hline $\mathrm{H}$ & 4.603579 & -3.253551 & 2.810490 \\
\hline $\mathrm{H}$ & 4.986689 & -4.141251 & -1.350800 \\
\hline $\mathrm{H}$ & 7.113650 & 0.117818 & -0.708260 \\
\hline $\mathrm{H}$ & 5.566471 & 4.098179 & -1.085490 \\
\hline $\mathrm{H}$ & 7.434050 & 2.497078 & -1.349920 \\
\hline $\mathrm{H}$ & 4.637491 & 5.104819 & 0.696980 \\
\hline $\mathrm{H}$ & 0.626242 & 6.608450 & 0.490780 \\
\hline $\mathrm{H}$ & 3.034202 & 6.967059 & 0.987790 \\
\hline $\mathrm{H}$ & -3.562839 & 4.419951 & 1.435850 \\
\hline $\mathrm{H}$ & -2.918469 & 4.114651 & -2.777010 \\
\hline $\mathrm{H}$ & 0.453340 & -0.804490 & -1.003050 \\
\hline $\mathrm{C}$ & 4.760660 & -0.580701 & 2.714860 \\
\hline $\mathrm{C}$ & 6.022500 & -0.765872 & 3.582620 \\
\hline $\mathrm{C}$ & 3.481900 & -0.708901 & 3.563980 \\
\hline $\mathrm{H}$ & 4.789850 & 0.442079 & 2.331080 \\
\hline $\mathrm{H}$ & 6.934800 & -0.633142 & 2.990060 \\
\hline $\mathrm{H}$ & 6.037320 & -0.032632 & 4.398220 \\
\hline $\mathrm{H}$ & 6.059819 & -1.765192 & 4.032320 \\
\hline $\mathrm{H}$ & 2.588110 & -0.521081 & 2.961950 \\
\hline $\mathrm{H}$ & 3.392179 & -1.705471 & 4.012800 \\
\hline $\mathrm{H}$ & 3.498520 & 0.020509 & 4.383030 \\
\hline $\mathrm{C}$ & 5.182039 & -1.663771 & -2.329300 \\
\hline $\mathrm{C}$ & 3.981039 & -2.076031 & -3.198950 \\
\hline $\mathrm{C}$ & 6.497089 & -2.225542 & -2.904620 \\
\hline $\mathrm{H}$ & 5.245960 & -0.575111 & -2.389230 \\
\hline $\mathrm{H}$ & 3.064689 & -1.597911 & -2.839810 \\
\hline $\mathrm{H}$ & 4.140209 & -1.766681 & -4.239270 \\
\hline $\mathrm{H}$ & 3.826209 & -3.161311 & -3.194200 \\
\hline $\mathrm{H}$ & 7.362539 & -1.910192 & -2.310520 \\
\hline $\mathrm{H}$ & 6.494149 & -3.321612 & -2.926350 \\
\hline $\mathrm{H}$ & 6.645269 & -1.874322 & -3.932960 \\
\hline $\mathrm{C}$ & 4.748468 & -5.358831 & 1.102440 \\
\hline $\mathrm{C}$ & 6.097818 & -6.032632 & 0.785050 \\
\hline $\mathrm{C}$ & 3.589348 & -6.097421 & 0.409280 \\
\hline $\mathrm{H}$ & 4.588048 & -5.441671 & 2.186200 \\
\hline
\end{tabular}




\begin{tabular}{|c|c|c|c|}
\hline $\mathrm{H}$ & 6.921118 & -5.537052 & 1.311040 \\
\hline $\mathrm{H}$ & 6.084758 & -7.087252 & 1.085810 \\
\hline $\mathrm{H}$ & 6.317078 & -5.994062 & -0.288710 \\
\hline $\mathrm{H}$ & 2.623528 & -5.649931 & 0.666440 \\
\hline $\mathrm{H}$ & 3.691448 & -6.067741 & -0.682090 \\
\hline $\mathrm{H}$ & 3.568818 & -7.151471 & 0.711440 \\
\hline $\mathrm{C}$ & -4.929239 & 4.251671 & -0.904260 \\
\hline $\mathrm{C}$ & -5.412689 & 3.086032 & -1.784830 \\
\hline $\mathrm{C}$ & -5.409469 & 5.602402 & -1.472330 \\
\hline $\mathrm{H}$ & -5.391269 & 4.129312 & 0.085290 \\
\hline $\mathrm{H}$ & -5.088630 & 2.120171 & -1.383240 \\
\hline $\mathrm{H}$ & -6.507399 & 3.080452 & -1.845050 \\
\hline $\mathrm{H}$ & -5.028749 & 3.168321 & -2.808570 \\
\hline $\mathrm{H}$ & -5.111778 & 6.433551 & -0.823710 \\
\hline $\mathrm{H}$ & -4.981349 & 5.784371 & -2.465520 \\
\hline $\mathrm{H}$ & -6.501899 & 5.617452 & -1.569160 \\
\hline $\mathrm{C}$ & -1.019929 & 4.587320 & 2.271410 \\
\hline $\mathrm{C}$ & -1.384319 & 3.364140 & 3.134140 \\
\hline $\mathrm{C}$ & -1.537269 & 5.889881 & 2.913050 \\
\hline $\mathrm{H}$ & 0.071051 & 4.644940 & 2.262690 \\
\hline $\mathrm{H}$ & -0.918860 & 2.458340 & 2.733490 \\
\hline $\mathrm{H}$ & -1.022709 & 3.504750 & 4.160330 \\
\hline $\mathrm{H}$ & -2.468909 & 3.209231 & 3.186280 \\
\hline $\mathrm{H}$ & -1.247708 & 6.769590 & 2.326660 \\
\hline $\mathrm{H}$ & -2.630429 & 5.893881 & 2.995990 \\
\hline $\mathrm{H}$ & -1.127919 & 6.006540 & 3.923740 \\
\hline $\mathrm{C}$ & -0.249079 & 4.233270 & -2.832330 \\
\hline $\mathrm{C}$ & -0.481229 & 5.481890 & -3.706810 \\
\hline $\mathrm{C}$ & -0.425139 & 2.940770 & -3.651620 \\
\hline $\mathrm{H}$ & 0.793451 & 4.266100 & -2.505510 \\
\hline $\mathrm{H}$ & -0.314878 & 6.402590 & -3.136230 \\
\hline $\mathrm{H}$ & 0.204821 & 5.483600 & -4.562540 \\
\hline $\mathrm{H}$ & -1.504119 & 5.514371 & -4.100420 \\
\hline $\mathrm{H}$ & -0.222290 & 2.057610 & -3.038760 \\
\hline $\mathrm{H}$ & -1.441419 & 2.853781 & -4.054930 \\
\hline $\mathrm{H}$ & 0.268141 & 2.932300 & -4.501490 \\
\hline $\mathrm{C}$ & -4.918581 & -2.217299 & 2.072780 \\
\hline $\mathrm{C}$ & -5.730391 & -2.642448 & 1.273000 \\
\hline $\mathrm{C}$ & -1.915440 & -0.683029 & 3.736550 \\
\hline $\mathrm{H}$ & -0.952290 & -0.259330 & 3.463350 \\
\hline $\mathrm{C}$ & -6.614021 & -3.148568 & 0.276720 \\
\hline $\mathrm{C}$ & -7.979261 & -3.352588 & 0.559700 \\
\hline $\mathrm{C}$ & -6.122791 & -3.452858 & -1.010670 \\
\hline $\mathrm{C}$ & -8.834001 & -3.846177 & -0.421950 \\
\hline $\mathrm{H}$ & -8.355481 & -3.119068 & 1.551310 \\
\hline $\mathrm{C}$ & -6.989041 & -3.946468 & -1.983020 \\
\hline $\mathrm{H}$ & -5.068251 & -3.306329 & -1.228670 \\
\hline $\mathrm{C}$ & -8.342491 & -4.143948 & -1.695590 \\
\hline $\mathrm{H}$ & -9.885471 & -3.999187 & -0.193560 \\
\hline $\mathrm{H}$ & -6.604041 & -4.179168 & -2.972420 \\
\hline $\mathrm{H}$ & -9.011761 & -4.529397 & -2.460300 \\
\hline
\end{tabular}

1d propargylboration TS 6 
B3LYP Energy =

$-3454.924703$

B3LYP Free Energy =

$-3453.722600$

M06-2X Derived Solution Free Energy (toluene) =

$-3453.239132$

Number of Imaginary Frequencies $=$

$1(-330.78)$

B3LYP/6-31G(d) Geometry

\begin{tabular}{|c|c|c|c|}
\hline $\mathrm{O}$ & -0.712940 & 4.761070 & -1.806789 \\
\hline B & -0.496060 & 3.659180 & -0.934729 \\
\hline $\mathrm{O}$ & -1.342150 & 3.780910 & 0.296161 \\
\hline $\mathrm{O}$ & -0.778950 & 2.440830 & -1.702209 \\
\hline $\mathrm{C}$ & -1.149370 & 3.208710 & 1.458501 \\
\hline $\mathrm{C}$ & -1.017710 & 2.821960 & -3.088999 \\
\hline $\mathrm{C}$ & -1.978750 & 3.753510 & 2.565561 \\
\hline $\mathrm{C}$ & -2.691310 & 4.943200 & 2.356601 \\
\hline $\mathrm{C}$ & -2.079990 & 3.093530 & 3.818811 \\
\hline $\mathrm{C}$ & -2.907870 & 3.653350 & 4.814341 \\
\hline $\mathrm{C}$ & -3.499580 & 5.481220 & 3.352631 \\
\hline $\mathrm{C}$ & -3.608940 & 4.831390 & 4.585921 \\
\hline $\mathrm{C}$ & -1.477650 & 4.316090 & -2.934559 \\
\hline $\mathrm{C}$ & -2.971870 & 4.449480 & -2.587869 \\
\hline $\mathrm{C}$ & -1.146670 & 5.212530 & -4.129439 \\
\hline $\mathrm{C}$ & -2.071790 & 1.889900 & -3.683549 \\
\hline $\mathrm{C}$ & 0.305450 & 2.674750 & -3.855749 \\
\hline $\mathrm{C}$ & 1.135390 & 3.712360 & -0.378499 \\
\hline $\mathrm{C}$ & 1.213590 & 3.664420 & 0.889941 \\
\hline $\mathrm{C}$ & 0.739710 & 3.632320 & 2.163361 \\
\hline $\mathrm{H}$ & -0.933070 & 2.137740 & 1.469051 \\
\hline $\mathrm{H}$ & -2.605350 & 5.427530 & 1.390441 \\
\hline $\mathrm{H}$ & -2.985180 & 3.142860 & 5.769011 \\
\hline $\mathrm{H}$ & -4.046750 & 6.401180 & 3.167491 \\
\hline $\mathrm{H}$ & -4.241060 & 5.242200 & 5.368351 \\
\hline $\mathrm{H}$ & -3.244080 & 3.807750 & -1.744919 \\
\hline $\mathrm{H}$ & -3.168880 & 5.486650 & -2.298239 \\
\hline $\mathrm{H}$ & -3.615990 & 4.202000 & -3.438969 \\
\hline $\mathrm{H}$ & -1.487280 & 6.232770 & -3.924079 \\
\hline $\mathrm{H}$ & -0.070830 & 5.250990 & -4.315929 \\
\hline $\mathrm{H}$ & -1.649670 & 4.864720 & -5.039579 \\
\hline $\mathrm{H}$ & -1.671370 & 0.878270 & -3.785589 \\
\hline $\mathrm{H}$ & -2.963300 & 1.837120 & -3.054669 \\
\hline $\mathrm{H}$ & -2.366710 & 2.235210 & -4.681669 \\
\hline $\mathrm{H}$ & 1.061860 & 3.372440 & -3.484229 \\
\hline $\mathrm{H}$ & 0.685630 & 1.655940 & -3.729289 \\
\hline $\mathrm{H}$ & 0.169310 & 2.856330 & -4.927319 \\
\hline $\mathrm{H}$ & 1.912610 & 3.801390 & -1.125429 \\
\hline $\mathrm{H}$ & 0.665840 & 4.569540 & 2.714161 \\
\hline $\mathrm{H}$ & 0.875950 & 2.742090 & 2.772441 \\
\hline $\mathrm{C}$ & -3.123980 & -2.780420 & -0.829109 \\
\hline $\mathrm{C}$ & -3.837930 & -2.258090 & 0.272181 \\
\hline $\mathrm{C}$ & -4.940680 & -1.431000 & 0.029481 \\
\hline $\mathrm{C}$ & -5.361100 & -1.102930 & -1.259869 \\
\hline $\mathrm{C}$ & -4.647280 & -1.643310 & -2.332819 \\
\hline $\mathrm{C}$ & -3.542410 & -2.481420 & -2.149839 \\
\hline $\mathrm{O}$ & -0.635700 & -1.908740 & 0.251831 \\
\hline
\end{tabular}




\begin{tabular}{|c|c|c|c|}
\hline $\mathrm{P}$ & 0.360950 & -0.728970 & -0.244469 \\
\hline $\mathrm{C}$ & -0.696980 & -3.224340 & -0.192389 \\
\hline $\mathrm{O}$ & 0.629840 & 0.198360 & 0.880851 \\
\hline $\mathrm{O}$ & 1.617010 & -1.559010 & -0.858079 \\
\hline $\mathrm{C}$ & 0.420240 & -4.072540 & -0.066749 \\
\hline $\mathrm{C}$ & -1.951370 & -3.693330 & -0.622559 \\
\hline $\mathrm{C}$ & 2.352040 & -2.420080 & -0.043989 \\
\hline $\mathrm{C}$ & 1.765880 & -3.626280 & 0.380771 \\
\hline $\mathrm{C}$ & -2.079820 & -5.061440 & -0.896319 \\
\hline $\mathrm{C}$ & 0.242350 & -5.429660 & -0.392189 \\
\hline $\mathrm{C}$ & -0.993860 & -5.926290 & -0.785989 \\
\hline $\mathrm{C}$ & 3.695130 & -2.094300 & 0.211611 \\
\hline $\mathrm{C}$ & 2.552750 & -4.481250 & 1.173861 \\
\hline $\mathrm{C}$ & 4.445600 & -3.002960 & 0.970451 \\
\hline $\mathrm{C}$ & 3.876260 & -4.173510 & 1.469371 \\
\hline $\mathrm{C}$ & 4.335960 & -0.844900 & -0.317499 \\
\hline $\mathrm{C}$ & 4.737620 & 0.175530 & 0.575881 \\
\hline $\mathrm{C}$ & 5.373290 & 1.311910 & 0.059241 \\
\hline $\mathrm{C}$ & 5.628020 & 1.475630 & -1.301529 \\
\hline $\mathrm{C}$ & 5.228970 & 0.449900 & -2.162959 \\
\hline $\mathrm{C}$ & 4.592650 & -0.706910 & -1.704639 \\
\hline $\mathrm{O}$ & -0.236080 & -0.107400 & -1.561449 \\
\hline $\mathrm{H}$ & -5.495720 & -1.031240 & 0.875461 \\
\hline $\mathrm{H}$ & -4.960120 & -1.409340 & -3.347589 \\
\hline $\mathrm{H}$ & -3.048320 & -5.436420 & -1.214599 \\
\hline $\mathrm{H}$ & 1.099280 & -6.093910 & -0.344929 \\
\hline $\mathrm{H}$ & -1.106930 & -6.980640 & -1.021469 \\
\hline $\mathrm{H}$ & 2.111750 & -5.397620 & 1.553301 \\
\hline $\mathrm{H}$ & 5.488480 & -2.775330 & 1.170441 \\
\hline $\mathrm{H}$ & 4.469270 & -4.852550 & 2.075521 \\
\hline $\mathrm{H}$ & 5.677950 & 2.099910 & 0.744451 \\
\hline $\mathrm{H}$ & 5.425620 & 0.547390 & -3.227789 \\
\hline $\mathrm{H}$ & -0.426430 & 0.895020 & -1.513949 \\
\hline $\mathrm{C}$ & -3.483250 & -2.606650 & 1.716541 \\
\hline $\mathrm{C}$ & -4.578970 & -3.482440 & 2.357221 \\
\hline $\mathrm{C}$ & -3.203000 & -1.357010 & 2.569641 \\
\hline $\mathrm{H}$ & -2.565550 & -3.201670 & 1.707691 \\
\hline $\mathrm{H}$ & -4.744060 & -4.398830 & 1.779081 \\
\hline $\mathrm{H}$ & -4.293290 & -3.769480 & 3.376531 \\
\hline $\mathrm{H}$ & -5.534980 & -2.948450 & 2.415911 \\
\hline $\mathrm{H}$ & -2.400040 & -0.755790 & 2.134001 \\
\hline $\mathrm{H}$ & -4.091750 & -0.722050 & 2.665351 \\
\hline $\mathrm{H}$ & -2.894860 & -1.647710 & 3.580531 \\
\hline $\mathrm{C}$ & -2.841880 & -3.045100 & -3.388939 \\
\hline $\mathrm{C}$ & -2.210620 & -1.938350 & -4.252509 \\
\hline $\mathrm{C}$ & -3.790430 & -3.925980 & -4.225629 \\
\hline $\mathrm{H}$ & -2.022160 & -3.686510 & -3.056279 \\
\hline $\mathrm{H}$ & -1.478560 & -1.368080 & -3.672859 \\
\hline $\mathrm{H}$ & -1.697850 & -2.375860 & -5.118139 \\
\hline $\mathrm{H}$ & -2.965750 & -1.239380 & -4.631039 \\
\hline $\mathrm{H}$ & -4.213450 & -4.740190 & -3.626089 \\
\hline $\mathrm{H}$ & -4.627040 & -3.346240 & -4.632709 \\
\hline $\mathrm{H}$ & -3.252470 & -4.370770 & -5.071569 \\
\hline
\end{tabular}




\begin{tabular}{|c|c|c|c|}
\hline $\mathrm{C}$ & -6.564300 & -0.194130 & -1.478219 \\
\hline $\mathrm{C}$ & -7.697660 & -0.909330 & -2.237569 \\
\hline $\mathrm{C}$ & -6.171440 & 1.118610 & -2.181919 \\
\hline $\mathrm{H}$ & -6.951460 & 0.070190 & -0.484589 \\
\hline $\mathrm{H}$ & -8.006160 & -1.823600 & -1.718849 \\
\hline $\mathrm{H}$ & -8.573820 & -0.256170 & -2.329919 \\
\hline $\mathrm{H}$ & -7.384390 & -1.189840 & -3.250249 \\
\hline $\mathrm{H}$ & -5.395190 & 1.650640 & -1.621039 \\
\hline $\mathrm{H}$ & -5.784970 & 0.928330 & -3.190349 \\
\hline $\mathrm{H}$ & -7.039420 & 1.782260 & -2.277599 \\
\hline $\mathrm{C}$ & 6.320500 & 2.729220 & -1.820399 \\
\hline $\mathrm{C}$ & 5.406040 & 3.543770 & -2.754729 \\
\hline $\mathrm{C}$ & 7.661200 & 2.405600 & -2.506399 \\
\hline $\mathrm{H}$ & 6.542270 & 3.359000 & -0.947819 \\
\hline $\mathrm{H}$ & 4.473280 & 3.821250 & -2.250669 \\
\hline $\mathrm{H}$ & 5.904160 & 4.465180 & -3.079519 \\
\hline $\mathrm{H}$ & 5.142310 & 2.971890 & -3.652189 \\
\hline $\mathrm{H}$ & 8.328510 & 1.857930 & -1.831989 \\
\hline $\mathrm{H}$ & 7.512880 & 1.790020 & -3.401499 \\
\hline $\mathrm{H}$ & 8.169110 & 3.326630 & -2.817019 \\
\hline $\mathrm{C}$ & 4.498130 & 0.103900 & 2.084301 \\
\hline $\mathrm{C}$ & 3.591170 & 1.249570 & 2.568351 \\
\hline $\mathrm{C}$ & 5.822360 & 0.070770 & 2.871761 \\
\hline $\mathrm{H}$ & 3.967530 & -0.826270 & 2.303781 \\
\hline $\mathrm{H}$ & 2.639550 & 1.228470 & 2.030791 \\
\hline $\mathrm{H}$ & 3.386950 & 1.143240 & 3.640891 \\
\hline $\mathrm{H}$ & 4.056280 & 2.230540 & 2.412411 \\
\hline $\mathrm{H}$ & 6.455960 & -0.768300 & 2.561171 \\
\hline $\mathrm{H}$ & 6.399640 & 0.991370 & 2.725921 \\
\hline $\mathrm{H}$ & 5.625710 & -0.031620 & 3.945781 \\
\hline $\mathrm{C}$ & 4.247340 & -1.794950 & -2.722119 \\
\hline $\mathrm{C}$ & 5.522590 & -2.406640 & -3.336499 \\
\hline $\mathrm{C}$ & 3.294020 & -1.283820 & -3.818839 \\
\hline $\mathrm{H}$ & 3.730570 & -2.603970 & -2.199299 \\
\hline $\mathrm{H}$ & 6.180170 & -2.815430 & -2.561009 \\
\hline $\mathrm{H}$ & 5.263780 & -3.218820 & -4.026669 \\
\hline $\mathrm{H}$ & 6.095390 & -1.660320 & -3.899429 \\
\hline $\mathrm{H}$ & 2.377230 & -0.877960 & -3.381139 \\
\hline $\mathrm{H}$ & 3.761280 & -0.500140 & -4.427009 \\
\hline $\mathrm{H}$ & 3.017680 & -2.103050 & -4.493879 \\
\hline $\mathrm{C}$ & -1.366690 & 1.900800 & 4.126701 \\
\hline $\mathrm{C}$ & -0.778200 & 0.895940 & 4.482851 \\
\hline $\mathrm{C}$ & -0.094980 & -0.275040 & 4.923351 \\
\hline $\mathrm{C}$ & 0.648250 & -1.058760 & 4.017231 \\
\hline $\mathrm{C}$ & -0.158240 & -0.658120 & 6.278861 \\
\hline $\mathrm{C}$ & 1.310600 & -2.198300 & 4.468731 \\
\hline $\mathrm{H}$ & 0.691340 & -0.763830 & 2.973151 \\
\hline $\mathrm{C}$ & 0.509650 & -1.797810 & 6.716781 \\
\hline $\mathrm{H}$ & -0.732080 & -0.053690 & 6.974951 \\
\hline $\mathrm{C}$ & 1.245650 & -2.570270 & 5.814121 \\
\hline $\mathrm{H}$ & 1.877040 & -2.800700 & 3.763581 \\
\hline $\mathrm{H}$ & 0.455910 & -2.084480 & 7.763661 \\
\hline $\mathrm{H}$ & 1.765310 & -3.460360 & 6.159101 \\
\hline
\end{tabular}


B3LYP Energy =

B3LYP Free Energy =

M06-2X Derived Solution Free Energy (toluene) = Number of Imaginary Frequencies $=$
$-3454.928601$

$-3453.726907$

$-3453.239050$

$1(-362.60)$

B3LYP/6-31G(d) Geometry

\begin{tabular}{|c|c|c|c|}
\hline B & 2.278870 & -0.666429 & -1.689040 \\
\hline $\mathrm{C}$ & 3.098139 & 0.857461 & -1.959110 \\
\hline $\mathrm{C}$ & 3.876839 & 1.102021 & -0.986870 \\
\hline $\mathrm{C}$ & 4.370209 & 0.909232 & 0.270710 \\
\hline $\mathrm{H}$ & 2.902889 & 1.375751 & -2.886780 \\
\hline $\mathrm{H}$ & 5.397559 & 0.579092 & 0.412930 \\
\hline $\mathrm{H}$ & 3.971179 & 1.526632 & 1.074790 \\
\hline $\mathrm{O}$ & 2.972380 & -1.684889 & -2.408580 \\
\hline $\mathrm{C}$ & 1.978480 & -2.554399 & -2.988890 \\
\hline $\mathrm{O}$ & 0.917250 & -0.623649 & -2.180400 \\
\hline $\mathrm{C}$ & 0.792870 & -1.561379 & -3.284820 \\
\hline $\mathrm{C}$ & 1.606551 & -3.629289 & -1.952760 \\
\hline $\mathrm{H}$ & 1.138881 & -3.189509 & -1.067230 \\
\hline $\mathrm{H}$ & 2.521771 & -4.140109 & -1.635960 \\
\hline $\mathrm{H}$ & 0.924541 & -4.378429 & -2.370670 \\
\hline $\mathrm{C}$ & 2.584111 & -3.214259 & -4.227760 \\
\hline $\mathrm{H}$ & 3.403051 & -3.875679 & -3.925970 \\
\hline $\mathrm{H}$ & 2.989500 & -2.471649 & -4.918770 \\
\hline $\mathrm{H}$ & 1.839141 & -3.819469 & -4.757580 \\
\hline $\mathrm{C}$ & -0.603000 & -2.181970 & -3.244820 \\
\hline $\mathrm{H}$ & -0.825870 & -2.621020 & -2.269840 \\
\hline $\mathrm{H}$ & -1.359850 & -1.417700 & -3.450740 \\
\hline $\mathrm{H}$ & -0.699110 & -2.962510 & -4.008490 \\
\hline $\mathrm{C}$ & 0.981860 & -0.774429 & -4.590130 \\
\hline $\mathrm{H}$ & 1.990670 & -0.356249 & -4.659320 \\
\hline $\mathrm{H}$ & 0.263480 & 0.051311 & -4.619370 \\
\hline $\mathrm{H}$ & 0.811140 & -1.405929 & -5.468660 \\
\hline $\mathrm{O}$ & 2.257350 & -0.807449 & -0.209820 \\
\hline $\mathrm{C}$ & 3.384880 & -0.874789 & 0.453450 \\
\hline $\mathrm{H}$ & 4.191460 & -1.433648 & -0.025690 \\
\hline $\mathrm{C}$ & 3.283620 & -0.989809 & 1.926200 \\
\hline $\mathrm{C}$ & 4.416840 & -1.376138 & 2.691290 \\
\hline $\mathrm{C}$ & 4.286760 & -1.486788 & 4.089000 \\
\hline $\mathrm{C}$ & 1.957210 & -0.856059 & 3.949190 \\
\hline $\mathrm{C}$ & 3.069980 & -1.225209 & 4.710890 \\
\hline $\mathrm{H}$ & 5.152430 & -1.784828 & 4.672100 \\
\hline $\mathrm{H}$ & 1.001930 & -0.665459 & 4.429080 \\
\hline $\mathrm{H}$ & 2.988240 & -1.317279 & 5.790490 \\
\hline $\mathrm{H}$ & -0.313430 & 0.160990 & -1.529680 \\
\hline $\mathrm{O}$ & -1.117091 & 0.715510 & -1.256030 \\
\hline $\mathrm{P}$ & -1.667740 & 0.458890 & 0.197840 \\
\hline $\mathrm{O}$ & -0.909310 & -0.434280 & 1.098080 \\
\hline $\mathrm{O}$ & -3.199080 & 0.028610 & -0.152300 \\
\hline $\mathrm{O}$ & -1.858051 & 1.917760 & 0.889910 \\
\hline $\mathrm{C}$ & -4.170210 & 0.039769 & 0.845740 \\
\hline
\end{tabular}




\begin{tabular}{|c|c|c|c|}
\hline $\mathrm{C}$ & -4.663671 & 1.278609 & 1.289060 \\
\hline $\mathrm{C}$ & -4.699870 & -1.187271 & 1.274870 \\
\hline $\mathrm{C}$ & -5.693331 & 1.264669 & 2.244480 \\
\hline $\mathrm{C}$ & -5.739590 & -1.146461 & 2.214210 \\
\hline $\mathrm{C}$ & -6.223750 & 0.064919 & 2.706770 \\
\hline $\mathrm{H}$ & -6.067251 & 2.209529 & 2.627190 \\
\hline $\mathrm{H}$ & -6.170890 & -2.083781 & 2.553070 \\
\hline $\mathrm{H}$ & -7.019910 & 0.072399 & 3.445870 \\
\hline $\mathrm{C}$ & -2.819201 & 2.850530 & 0.517510 \\
\hline $\mathrm{C}$ & -2.371391 & 4.103180 & 0.066950 \\
\hline $\mathrm{C}$ & -4.181391 & 2.569629 & 0.734650 \\
\hline $\mathrm{C}$ & -3.339552 & 5.083580 & -0.193140 \\
\hline $\mathrm{C}$ & -5.111061 & 3.580659 & 0.439690 \\
\hline $\mathrm{C}$ & -4.698042 & 4.825879 & -0.020280 \\
\hline $\mathrm{H}$ & -3.008192 & 6.061990 & -0.528320 \\
\hline $\mathrm{H}$ & -6.168711 & 3.370999 & 0.567520 \\
\hline $\mathrm{H}$ & -5.432022 & 5.595799 & -0.240660 \\
\hline $\mathrm{C}$ & -4.223310 & -2.502151 & 0.728010 \\
\hline $\mathrm{C}$ & -3.411639 & -3.349410 & 1.516360 \\
\hline $\mathrm{C}$ & -4.637710 & -2.918771 & -0.560360 \\
\hline $\mathrm{C}$ & -3.043449 & -4.598180 & 1.000120 \\
\hline $\mathrm{C}$ & -4.233409 & -4.172631 & -1.028280 \\
\hline $\mathrm{C}$ & -3.439139 & -5.032780 & -0.264610 \\
\hline $\mathrm{H}$ & -2.423389 & -5.255530 & 1.605250 \\
\hline $\mathrm{H}$ & -4.558109 & -4.489881 & -2.016490 \\
\hline $\mathrm{C}$ & -0.917892 & 4.447030 & -0.096940 \\
\hline $\mathrm{C}$ & -0.360302 & 4.568120 & -1.392970 \\
\hline $\mathrm{C}$ & -0.131202 & 4.749510 & 1.037640 \\
\hline $\mathrm{C}$ & 0.953998 & 5.029271 & -1.523000 \\
\hline $\mathrm{C}$ & 1.183388 & 5.192661 & 0.848940 \\
\hline $\mathrm{C}$ & 1.743888 & 5.356871 & -0.418000 \\
\hline $\mathrm{H}$ & 1.370038 & 5.140711 & -2.521100 \\
\hline $\mathrm{H}$ & 1.787278 & 5.436151 & 1.720710 \\
\hline $\mathrm{C}$ & -3.025579 & -6.405000 & -0.781190 \\
\hline $\mathrm{C}$ & -2.182479 & -6.310860 & -2.066380 \\
\hline $\mathrm{C}$ & -4.242208 & -7.327901 & -0.985940 \\
\hline $\mathrm{H}$ & -2.393608 & -6.862850 & -0.007790 \\
\hline $\mathrm{H}$ & -1.293879 & -5.689330 & -1.909800 \\
\hline $\mathrm{H}$ & -1.851998 & -7.306450 & -2.386700 \\
\hline $\mathrm{H}$ & -2.757519 & -5.870330 & -2.889540 \\
\hline $\mathrm{H}$ & -4.821778 & -7.428501 & -0.061750 \\
\hline $\mathrm{H}$ & -4.913808 & -6.935211 & -1.758810 \\
\hline $\mathrm{H}$ & -3.922128 & -8.329021 & -1.299700 \\
\hline $\mathrm{C}$ & -2.935920 & -2.963950 & 2.917160 \\
\hline $\mathrm{C}$ & -1.412300 & -3.113340 & 3.083280 \\
\hline $\mathrm{C}$ & -3.685319 & -3.767541 & 3.999120 \\
\hline $\mathrm{H}$ & -3.166740 & -1.905730 & 3.071460 \\
\hline $\mathrm{H}$ & -0.883660 & -2.520050 & 2.333540 \\
\hline $\mathrm{H}$ & -1.110380 & -2.760050 & 4.077330 \\
\hline $\mathrm{H}$ & -1.090689 & -4.158480 & 2.999830 \\
\hline $\mathrm{H}$ & -4.769519 & -3.621571 & 3.934820 \\
\hline $\mathrm{H}$ & -3.489469 & -4.842171 & 3.899680 \\
\hline $\mathrm{H}$ & -3.361299 & -3.459600 & 5.000920 \\
\hline
\end{tabular}




\begin{tabular}{|c|c|c|c|}
\hline $\mathrm{C}$ & -5.540450 & -2.062561 & -1.448850 \\
\hline $\mathrm{C}$ & -6.900870 & -2.745451 & -1.693110 \\
\hline $\mathrm{C}$ & -4.857870 & -1.691651 & -2.779010 \\
\hline $\mathrm{H}$ & -5.747070 & -1.126011 & -0.923570 \\
\hline $\mathrm{H}$ & -7.405840 & -2.971842 & -0.747270 \\
\hline $\mathrm{H}$ & -7.557320 & -2.093162 & -2.281920 \\
\hline $\mathrm{H}$ & -6.787199 & -3.687011 & -2.243210 \\
\hline $\mathrm{H}$ & -3.920940 & -1.155761 & -2.598770 \\
\hline $\mathrm{H}$ & -4.633800 & -2.581591 & -3.379290 \\
\hline $\mathrm{H}$ & -5.512290 & -1.045141 & -3.376600 \\
\hline $\mathrm{C}$ & 3.153108 & 5.914621 & -0.577170 \\
\hline $\mathrm{C}$ & 3.123088 & 7.339951 & -1.163620 \\
\hline $\mathrm{C}$ & 4.068248 & 4.999972 & -1.410530 \\
\hline $\mathrm{H}$ & 3.587028 & 5.983571 & 0.430020 \\
\hline $\mathrm{H}$ & 2.510207 & 8.007561 & -0.548220 \\
\hline $\mathrm{H}$ & 4.134948 & 7.759452 & -1.221320 \\
\hline $\mathrm{H}$ & 2.701668 & 7.339391 & -2.175990 \\
\hline $\mathrm{H}$ & 4.120839 & 3.992652 & -0.983680 \\
\hline $\mathrm{H}$ & 3.709728 & 4.906631 & -2.442570 \\
\hline $\mathrm{H}$ & 5.085638 & 5.407452 & -1.451100 \\
\hline $\mathrm{C}$ & -1.148271 & 4.225680 & -2.658210 \\
\hline $\mathrm{C}$ & -0.392891 & 3.239520 & -3.569460 \\
\hline $\mathrm{C}$ & -1.537072 & 5.494860 & -3.442770 \\
\hline $\mathrm{H}$ & -2.073821 & 3.728010 & -2.353730 \\
\hline $\mathrm{H}$ & -0.119511 & 2.333370 & -3.022380 \\
\hline $\mathrm{H}$ & -1.028001 & 2.949960 & -4.415700 \\
\hline $\mathrm{H}$ & 0.520249 & 3.683421 & -3.984160 \\
\hline $\mathrm{H}$ & -2.131202 & 6.181890 & -2.830480 \\
\hline $\mathrm{H}$ & -0.646602 & 6.038240 & -3.781950 \\
\hline $\mathrm{H}$ & -2.128542 & 5.234720 & -4.329140 \\
\hline $\mathrm{C}$ & -0.670132 & 4.645360 & 2.463680 \\
\hline $\mathrm{C}$ & -0.738312 & 6.027680 & 3.142520 \\
\hline $\mathrm{C}$ & 0.139799 & 3.647380 & 3.312890 \\
\hline $\mathrm{H}$ & -1.693771 & 4.264670 & 2.414470 \\
\hline $\mathrm{H}$ & -1.354132 & 6.723960 & 2.562060 \\
\hline $\mathrm{H}$ & -1.174892 & 5.942170 & 4.145110 \\
\hline $\mathrm{H}$ & 0.257678 & 6.473481 & 3.249800 \\
\hline $\mathrm{H}$ & 0.153099 & 2.657580 & 2.846370 \\
\hline $\mathrm{H}$ & 1.177219 & 3.977491 & 3.445970 \\
\hline $\mathrm{H}$ & -0.305421 & 3.548960 & 4.310540 \\
\hline $\mathrm{C}$ & 5.673040 & -1.658098 & 2.080970 \\
\hline $\mathrm{C}$ & 6.751410 & -1.909338 & 1.574100 \\
\hline $\mathrm{C}$ & 2.062020 & -0.739159 & 2.564790 \\
\hline $\mathrm{H}$ & 1.191380 & -0.480199 & 1.969000 \\
\hline $\mathrm{C}$ & 8.014790 & -2.207617 & 0.985780 \\
\hline $\mathrm{C}$ & 9.141090 & -2.450007 & 1.797980 \\
\hline $\mathrm{C}$ & 8.158250 & -2.266027 & -0.415380 \\
\hline $\mathrm{C}$ & 10.373800 & -2.741037 & 1.220970 \\
\hline $\mathrm{H}$ & 9.034190 & -2.406707 & 2.877470 \\
\hline $\mathrm{C}$ & 9.395460 & -2.557727 & -0.982660 \\
\hline $\mathrm{H}$ & 7.293140 & -2.085078 & -1.046150 \\
\hline $\mathrm{C}$ & 10.506060 & -2.795717 & -0.168940 \\
\hline $\mathrm{H}$ & 11.234580 & -2.926187 & 1.857690 \\
\hline
\end{tabular}




$\begin{array}{lrrr}\mathrm{H} & 9.493100 & -2.600717 & -2.063930 \\ \mathrm{H} & 11.469830 & -3.023506 & -0.615800\end{array}$

\section{1d propargylboration TS 8}

B3LYP Energy =

$-3454.917277$

B3LYP Free Energy =

$-3453.714608$

M06-2X Derived Solution Free Energy (toluene) =

$-3453.230503$

Number of Imaginary Frequencies $=$

$1(-361.27)$

B3LYP/6-31G(d) Geometry

\begin{tabular}{|c|c|c|c|}
\hline B & -1.832251 & 2.341229 & -1.798420 \\
\hline $\mathrm{O}$ & -1.802541 & 3.018799 & -3.058470 \\
\hline $\mathrm{C}$ & -0.446882 & 3.461840 & -3.264500 \\
\hline $\mathrm{O}$ & -0.487031 & 1.885710 & -1.507180 \\
\hline $\mathrm{C}$ & 0.384279 & 2.297230 & -2.610530 \\
\hline $\mathrm{C}$ & -0.242262 & 4.811550 & -2.552040 \\
\hline $\mathrm{H}$ & -0.368972 & 4.722810 & -1.469820 \\
\hline $\mathrm{H}$ & -0.986232 & 5.523600 & -2.925160 \\
\hline $\mathrm{H}$ & 0.752538 & 5.224070 & -2.752400 \\
\hline $\mathrm{C}$ & -0.230282 & 3.643230 & -4.767660 \\
\hline $\mathrm{H}$ & -0.852482 & 4.469600 & -5.127420 \\
\hline $\mathrm{H}$ & -0.510561 & 2.746430 & -5.324160 \\
\hline $\mathrm{H}$ & 0.815058 & 3.886160 & -4.991290 \\
\hline $\mathrm{C}$ & 1.730349 & 2.705691 & -2.016510 \\
\hline $\mathrm{H}$ & 1.610848 & 3.448711 & -1.225030 \\
\hline $\mathrm{H}$ & 2.235939 & 1.836351 & -1.584070 \\
\hline $\mathrm{H}$ & 2.386269 & 3.118511 & -2.791740 \\
\hline $\mathrm{C}$ & 0.566019 & 1.109250 & -3.566280 \\
\hline $\mathrm{H}$ & -0.388870 & 0.804930 & -4.005390 \\
\hline $\mathrm{H}$ & 0.984140 & 0.255170 & -3.027870 \\
\hline $\mathrm{H}$ & 1.253949 & 1.363420 & -4.379340 \\
\hline $\mathrm{O}$ & -2.380261 & 3.200519 & -0.685950 \\
\hline $\mathrm{C}$ & -3.531762 & 3.765469 & -0.956060 \\
\hline $\mathrm{H}$ & -3.671752 & 4.024098 & -2.008690 \\
\hline $\mathrm{C}$ & -4.127522 & 4.755108 & -0.025060 \\
\hline $\mathrm{C}$ & -4.777902 & 5.841328 & -0.631400 \\
\hline $\mathrm{C}$ & -4.089402 & 4.675568 & 1.391120 \\
\hline $\mathrm{C}$ & -5.364803 & 6.854968 & 0.119440 \\
\hline $\mathrm{C}$ & -4.691392 & 5.713008 & 2.135660 \\
\hline $\mathrm{C}$ & -5.316303 & 6.787618 & 1.513740 \\
\hline $\mathrm{H}$ & -4.806733 & 5.893318 & -1.717140 \\
\hline $\mathrm{H}$ & -5.850733 & 7.689348 & -0.377550 \\
\hline $\mathrm{H}$ & -4.658032 & 5.650018 & 3.218510 \\
\hline $\mathrm{H}$ & -5.767643 & 7.570328 & 2.117120 \\
\hline $\mathrm{H}$ & 6.220510 & -1.341898 & 2.055910 \\
\hline $\mathrm{C}$ & 5.355841 & -1.893878 & 1.700030 \\
\hline $\mathrm{C}$ & 4.388110 & -1.207388 & 0.952120 \\
\hline $\mathrm{C}$ & 5.230601 & -3.253498 & 1.979460 \\
\hline $\mathrm{C}$ & 3.286381 & -1.945359 & 0.491420 \\
\hline $\mathrm{C}$ & 4.147281 & -3.964998 & 1.477150 \\
\hline $\mathrm{H}$ & 5.983971 & -3.759048 & 2.576880 \\
\hline $\mathrm{C}$ & 3.161011 & -3.329829 & 0.704230 \\
\hline
\end{tabular}




\begin{tabular}{|c|c|c|c|}
\hline $\mathrm{O}$ & 2.324990 & -1.306309 & -0.297700 \\
\hline $\mathrm{H}$ & 4.047912 & -5.025968 & 1.685000 \\
\hline $\mathrm{C}$ & 2.086232 & -4.140829 & 0.076080 \\
\hline $\mathrm{P}$ & 0.845910 & -1.069770 & 0.353140 \\
\hline $\mathrm{C}$ & 0.737621 & -3.741890 & 0.030530 \\
\hline $\mathrm{C}$ & 2.416852 & -5.387119 & -0.483030 \\
\hline $\mathrm{O}$ & 0.336551 & -2.575120 & 0.672360 \\
\hline $\mathrm{O}$ & 0.800930 & -0.223080 & 1.563370 \\
\hline $\mathrm{O}$ & 0.003600 & -0.616710 & -0.902420 \\
\hline $\mathrm{C}$ & -0.268618 & -4.556900 & -0.516020 \\
\hline $\mathrm{C}$ & 1.442792 & -6.214109 & -1.029940 \\
\hline $\mathrm{H}$ & 3.457832 & -5.695089 & -0.490280 \\
\hline $\mathrm{H}$ & -0.171420 & 0.380360 & -0.999570 \\
\hline $\mathrm{C}$ & 0.112252 & -5.801800 & -1.036010 \\
\hline $\mathrm{H}$ & 1.719173 & -7.176119 & -1.452000 \\
\hline $\mathrm{H}$ & -0.657468 & -6.446610 & -1.450260 \\
\hline $\mathrm{C}$ & 4.619170 & 0.239512 & 0.617500 \\
\hline $\mathrm{C}$ & 5.203570 & 0.575982 & -0.627610 \\
\hline $\mathrm{C}$ & 4.365759 & 1.248352 & 1.574870 \\
\hline $\mathrm{C}$ & 5.532779 & 1.911512 & -0.882100 \\
\hline $\mathrm{C}$ & 4.725169 & 2.567232 & 1.269660 \\
\hline $\mathrm{C}$ & 5.314419 & 2.924142 & 0.056250 \\
\hline $\mathrm{H}$ & 5.990229 & 2.162302 & -1.836320 \\
\hline $\mathrm{H}$ & 4.545129 & 3.344822 & 2.007850 \\
\hline $\mathrm{C}$ & -1.714408 & -4.154571 & -0.545240 \\
\hline $\mathrm{C}$ & -2.321179 & -3.798701 & -1.774480 \\
\hline $\mathrm{C}$ & -2.490388 & -4.200931 & 0.634930 \\
\hline $\mathrm{C}$ & -3.685979 & -3.491052 & -1.790720 \\
\hline $\mathrm{C}$ & -3.851129 & -3.882092 & 0.560590 \\
\hline $\mathrm{C}$ & -4.472599 & -3.524982 & -0.636250 \\
\hline $\mathrm{H}$ & -4.148329 & -3.223562 & -2.738110 \\
\hline $\mathrm{H}$ & -4.450849 & -3.922752 & 1.467230 \\
\hline $\mathrm{C}$ & 5.728888 & 4.365802 & -0.212010 \\
\hline $\mathrm{C}$ & 5.018318 & 4.963932 & -1.439820 \\
\hline $\mathrm{C}$ & 7.258528 & 4.498623 & -0.343540 \\
\hline $\mathrm{H}$ & 5.422688 & 4.955562 & 0.662900 \\
\hline $\mathrm{H}$ & 3.929828 & 4.915172 & -1.329910 \\
\hline $\mathrm{H}$ & 5.301607 & 6.014722 & -1.576190 \\
\hline $\mathrm{H}$ & 5.286568 & 4.426842 & -2.357510 \\
\hline $\mathrm{H}$ & 7.767228 & 4.109203 & 0.545110 \\
\hline $\mathrm{H}$ & 7.631938 & 3.942623 & -1.211910 \\
\hline $\mathrm{H}$ & 7.547688 & 5.549033 & -0.470400 \\
\hline $\mathrm{C}$ & 3.752759 & 0.941881 & 2.941420 \\
\hline $\mathrm{C}$ & 2.608809 & 1.903131 & 3.311460 \\
\hline $\mathrm{C}$ & 4.831409 & 0.937012 & 4.044130 \\
\hline $\mathrm{H}$ & 3.316070 & -0.059909 & 2.894430 \\
\hline $\mathrm{H}$ & 1.841849 & 1.912121 & 2.533990 \\
\hline $\mathrm{H}$ & 2.134959 & 1.574641 & 4.243640 \\
\hline $\mathrm{H}$ & 2.966339 & 2.927631 & 3.470720 \\
\hline $\mathrm{H}$ & 5.618630 & 0.202952 & 3.840270 \\
\hline $\mathrm{H}$ & 5.308609 & 1.920832 & 4.132870 \\
\hline $\mathrm{H}$ & 4.385300 & 0.691962 & 5.015790 \\
\hline $\mathrm{C}$ & 5.526110 & -0.473808 & -1.692040 \\
\hline
\end{tabular}




\begin{tabular}{|c|c|c|c|}
\hline $\mathrm{C}$ & 7.042870 & -0.571307 & -1.948140 \\
\hline $\mathrm{C}$ & 4.758160 & -0.221108 & -3.003150 \\
\hline $\mathrm{H}$ & 5.204480 & -1.449648 & -1.317670 \\
\hline $\mathrm{H}$ & 7.587040 & -0.794597 & -1.023610 \\
\hline $\mathrm{H}$ & 7.259790 & -1.367047 & -2.671220 \\
\hline $\mathrm{H}$ & 7.444710 & 0.364993 & -2.353010 \\
\hline $\mathrm{H}$ & 3.677450 & -0.217869 & -2.828520 \\
\hline $\mathrm{H}$ & 5.032630 & 0.740502 & -3.452830 \\
\hline $\mathrm{H}$ & 4.982520 & -1.006298 & -3.735460 \\
\hline $\mathrm{C}$ & -1.913068 & -4.639541 & 1.979610 \\
\hline $\mathrm{C}$ & -2.496268 & -5.999391 & 2.414190 \\
\hline $\mathrm{C}$ & -2.109079 & -3.576221 & 3.075230 \\
\hline $\mathrm{H}$ & -0.835528 & -4.780360 & 1.858610 \\
\hline $\mathrm{H}$ & -2.314557 & -6.770081 & 1.656460 \\
\hline $\mathrm{H}$ & -2.037588 & -6.331101 & 3.353690 \\
\hline $\mathrm{H}$ & -3.579338 & -5.938192 & 2.574640 \\
\hline $\mathrm{H}$ & -1.667169 & -2.620141 & 2.780790 \\
\hline $\mathrm{H}$ & -3.170099 & -3.406201 & 3.293300 \\
\hline $\mathrm{H}$ & -1.630439 & -3.900621 & 4.007360 \\
\hline $\mathrm{C}$ & -1.546559 & -3.751071 & -3.092990 \\
\hline $\mathrm{C}$ & -1.582609 & -2.352461 & -3.735820 \\
\hline $\mathrm{C}$ & -2.046098 & -4.823881 & -4.080640 \\
\hline $\mathrm{H}$ & -0.497079 & -3.971200 & -2.879330 \\
\hline $\mathrm{H}$ & -1.156059 & -1.609061 & -3.055780 \\
\hline $\mathrm{H}$ & -0.994599 & -2.341910 & -4.661770 \\
\hline $\mathrm{H}$ & -2.604789 & -2.048061 & -3.991720 \\
\hline $\mathrm{H}$ & -1.980598 & -5.828281 & -3.647250 \\
\hline $\mathrm{H}$ & -3.091918 & -4.653721 & -4.362940 \\
\hline $\mathrm{H}$ & -1.446358 & -4.810711 & -4.998840 \\
\hline $\mathrm{C}$ & -5.961349 & -3.204362 & -0.673790 \\
\hline $\mathrm{C}$ & -6.743148 & -4.223053 & -1.525010 \\
\hline $\mathrm{C}$ & -6.232239 & -1.765043 & -1.149150 \\
\hline $\mathrm{H}$ & -6.332769 & -3.284113 & 0.357060 \\
\hline $\mathrm{H}$ & -6.589968 & -5.244143 & -1.158900 \\
\hline $\mathrm{H}$ & -7.818249 & -4.007473 & -1.498280 \\
\hline $\mathrm{H}$ & -6.423088 & -4.193593 & -2.573450 \\
\hline $\mathrm{H}$ & -5.701630 & -1.036912 & -0.526280 \\
\hline $\mathrm{H}$ & -5.904809 & -1.618662 & -2.185410 \\
\hline $\mathrm{H}$ & -7.304480 & -1.538083 & -1.104930 \\
\hline $\mathrm{C}$ & -3.517602 & 3.593229 & 2.113840 \\
\hline $\mathrm{C}$ & -3.093621 & 2.749019 & 2.881460 \\
\hline $\mathrm{C}$ & -2.585331 & 1.773759 & 3.786620 \\
\hline $\mathrm{C}$ & -3.152151 & 1.638759 & 5.071210 \\
\hline $\mathrm{C}$ & -1.503351 & 0.950949 & 3.421250 \\
\hline $\mathrm{C}$ & -2.645900 & 0.699419 & 5.964150 \\
\hline $\mathrm{H}$ & -3.985951 & 2.274918 & 5.353390 \\
\hline $\mathrm{C}$ & -0.999620 & 0.018380 & 4.324970 \\
\hline $\mathrm{H}$ & -1.050251 & 1.043890 & 2.441170 \\
\hline $\mathrm{C}$ & -1.568450 & -0.111601 & 5.594420 \\
\hline $\mathrm{H}$ & -3.089980 & 0.600939 & 6.951280 \\
\hline $\mathrm{H}$ & -0.159940 & -0.597460 & 4.019580 \\
\hline $\mathrm{H}$ & -1.173340 & -0.841411 & 6.296380 \\
\hline $\mathrm{C}$ & -4.108071 & 1.420328 & -1.527170 \\
\hline
\end{tabular}




$\begin{array}{lrrr}\mathrm{C} & -2.955271 & 1.009359 & -1.868950 \\ \mathrm{H} & -2.602310 & 0.040819 & -2.195800 \\ \mathrm{C} & -4.972891 & 2.320598 & -0.976140 \\ \mathrm{H} & -5.238081 & 2.196548 & 0.073580 \\ \mathrm{H} & -5.738801 & 2.787598 & -1.593700\end{array}$

\section{1a allylboration TS 7 (TS-2Si-A)}

\section{B3LYP Energy =}

B3LYP Free Energy =

M06-2X Derived Solution Free Energy $(\mathrm{DCM})=$

$-3225.111022$

$-3223.962992$

$-3223.524279$

M06-2X Derived Solution Free Energy (toluene) $=$ Number of Imaginary Frequencies $=$

$-3223.515413$

$1(-326.08)$

B3LYP/6-31G(d) Geometry

\begin{tabular}{|c|c|c|c|}
\hline B & -1.186210 & 2.951590 & -1.436140 \\
\hline $\mathrm{C}$ & -2.891990 & 2.598070 & -1.547060 \\
\hline $\mathrm{C}$ & -3.505570 & 3.651960 & -0.792380 \\
\hline $\mathrm{C}$ & -3.578310 & 3.648100 & 0.586270 \\
\hline $\mathrm{H}$ & -3.095570 & 2.623170 & -2.618900 \\
\hline $\mathrm{H}$ & -3.025690 & 1.595530 & -1.134200 \\
\hline $\mathrm{H}$ & -3.682470 & 4.596520 & -1.308170 \\
\hline $\mathrm{H}$ & -3.985360 & 4.501340 & 1.120270 \\
\hline $\mathrm{H}$ & -3.571990 & 2.703240 & 1.122430 \\
\hline $\mathrm{O}$ & -0.862200 & 4.176520 & -2.095090 \\
\hline $\mathrm{C}$ & 0.418440 & 4.003340 & -2.733680 \\
\hline $\mathrm{O}$ & -0.371210 & 1.906730 & -2.024870 \\
\hline $\mathrm{C}$ & 0.404230 & 2.473500 & -3.114900 \\
\hline $\mathrm{C}$ & 1.524910 & 4.352870 & -1.722690 \\
\hline $\mathrm{H}$ & 1.518150 & 3.671500 & -0.867050 \\
\hline $\mathrm{H}$ & 1.351470 & 5.368390 & -1.351220 \\
\hline $\mathrm{H}$ & 2.519560 & 4.323760 & -2.181510 \\
\hline $\mathrm{C}$ & 0.490400 & 4.958250 & -3.926050 \\
\hline $\mathrm{H}$ & 0.481230 & 5.992560 & -3.566210 \\
\hline $\mathrm{H}$ & -0.362990 & 4.828830 & -4.595960 \\
\hline $\mathrm{H}$ & 1.413270 & 4.808510 & -4.498560 \\
\hline $\mathrm{C}$ & 1.774410 & 1.797000 & -3.141800 \\
\hline $\mathrm{H}$ & 2.277930 & 1.859870 & -2.174660 \\
\hline $\mathrm{H}$ & 1.663660 & 0.737350 & -3.395490 \\
\hline $\mathrm{H}$ & 2.417800 & 2.256890 & -3.900890 \\
\hline $\mathrm{C}$ & -0.349000 & 2.187260 & -4.421850 \\
\hline $\mathrm{H}$ & -1.323410 & 2.684190 & -4.433570 \\
\hline $\mathrm{H}$ & -0.512860 & 1.108690 & -4.513490 \\
\hline $\mathrm{H}$ & 0.220130 & 2.521160 & -5.296160 \\
\hline $\mathrm{O}$ & -0.961190 & 2.946630 & 0.051110 \\
\hline $\mathrm{C}$ & -1.437470 & 3.902610 & 0.786610 \\
\hline $\mathrm{H}$ & -1.441360 & 4.908540 & 0.368150 \\
\hline $\mathrm{C}$ & -1.297830 & 3.776250 & 2.249770 \\
\hline $\mathrm{C}$ & -1.606750 & 4.872320 & 3.097000 \\
\hline $\mathrm{C}$ & -0.818020 & 2.581210 & 2.805070 \\
\hline $\mathrm{C}$ & -1.434880 & 4.732990 & 4.484910 \\
\hline $\mathrm{C}$ & -0.653910 & 2.464960 & 4.183450 \\
\hline $\mathrm{C}$ & -0.965370 & 3.537820 & 5.023370 \\
\hline
\end{tabular}




\begin{tabular}{|c|c|c|c|}
\hline $\mathrm{H}$ & -1.672300 & 5.573750 & 5.128980 \\
\hline $\mathrm{H}$ & -0.272620 & 1.536430 & 4.597790 \\
\hline $\mathrm{H}$ & -0.836200 & 3.447380 & 6.098350 \\
\hline $\mathrm{H}$ & 5.160720 & -3.284050 & 2.163860 \\
\hline $\mathrm{C}$ & 4.141190 & -3.461320 & 1.834260 \\
\hline $\mathrm{C}$ & 3.527660 & -2.508620 & 1.009050 \\
\hline $\mathrm{C}$ & 3.470020 & -4.618610 & 2.227250 \\
\hline $\mathrm{C}$ & 2.214870 & -2.770510 & 0.586260 \\
\hline $\mathrm{C}$ & 2.175530 & -4.857320 & 1.778110 \\
\hline $\mathrm{H}$ & 3.959030 & -5.336150 & 2.880080 \\
\hline $\mathrm{C}$ & 1.523930 & -3.944850 & 0.931760 \\
\hline $\mathrm{O}$ & 1.622520 & -1.888160 & -0.313260 \\
\hline $\mathrm{H}$ & 1.648890 & -5.755080 & 2.087400 \\
\hline $\mathrm{C}$ & 0.179380 & -4.267040 & 0.388350 \\
\hline $\mathrm{P}$ & 0.369180 & -0.955140 & 0.144350 \\
\hline $\mathrm{C}$ & -0.858430 & -3.320120 & 0.301380 \\
\hline $\mathrm{C}$ & -0.099960 & -5.579580 & -0.027190 \\
\hline $\mathrm{O}$ & -0.656340 & -2.035890 & 0.794630 \\
\hline $\mathrm{O}$ & 0.651840 & 0.121780 & 1.116310 \\
\hline $\mathrm{O}$ & -0.217050 & -0.569120 & -1.266020 \\
\hline $\mathrm{C}$ & -2.150100 & -3.654250 & -0.137200 \\
\hline $\mathrm{C}$ & -1.366130 & -5.937500 & -0.476410 \\
\hline $\mathrm{H}$ & 0.696901 & -6.316610 & -0.002010 \\
\hline $\mathrm{H}$ & -0.239040 & 0.422460 & -1.486800 \\
\hline $\mathrm{C}$ & -2.381190 & -4.983660 & -0.518660 \\
\hline $\mathrm{H}$ & -1.563509 & -6.958410 & -0.790790 \\
\hline $\mathrm{H}$ & -3.378170 & -5.263090 & -0.846130 \\
\hline C & 4.280320 & -1.284040 & 0.574570 \\
\hline $\mathrm{C}$ & 4.829460 & -1.220240 & -0.728940 \\
\hline $\mathrm{C}$ & 4.488410 & -0.219140 & 1.480610 \\
\hline $\mathrm{C}$ & 5.571210 & -0.093590 & -1.096310 \\
\hline $\mathrm{C}$ & 5.249680 & 0.879560 & 1.062590 \\
\hline $\mathrm{C}$ & 5.798720 & 0.968360 & -0.216410 \\
\hline $\mathrm{H}$ & 5.992560 & -0.050590 & -2.097880 \\
\hline $\mathrm{H}$ & 5.418400 & 1.698130 & 1.758610 \\
\hline $\mathrm{C}$ & -3.284910 & -2.670310 & -0.169330 \\
\hline $\mathrm{C}$ & -3.773890 & -2.192570 & -1.409870 \\
\hline $\mathrm{C}$ & -3.929940 & -2.294610 & 1.031240 \\
\hline $\mathrm{C}$ & -4.915850 & -1.383531 & -1.420660 \\
\hline $\mathrm{C}$ & -5.061210 & -1.472981 & 0.961860 \\
\hline $\mathrm{C}$ & -5.584330 & -1.017511 & -0.248950 \\
\hline $\mathrm{H}$ & -5.298760 & -1.037961 & -2.377450 \\
\hline $\mathrm{H}$ & -5.565240 & -1.193911 & 1.884880 \\
\hline $\mathrm{C}$ & 6.622840 & 2.182140 & -0.626720 \\
\hline $\mathrm{C}$ & 5.971140 & 2.953820 & -1.789530 \\
\hline $\mathrm{C}$ & 8.077050 & 1.801740 & -0.963630 \\
\hline $\mathrm{H}$ & 6.652780 & 2.857950 & 0.239000 \\
\hline $\mathrm{H}$ & 4.951350 & 3.262850 & -1.534640 \\
\hline $\mathrm{H}$ & 6.550700 & 3.852970 & -2.032170 \\
\hline $\mathrm{H}$ & 5.917140 & 2.337830 & -2.695150 \\
\hline $\mathrm{H}$ & 8.555020 & 1.284880 & -0.124250 \\
\hline $\mathrm{H}$ & 8.123170 & 1.136680 & -1.834250 \\
\hline $\mathrm{H}$ & 8.667980 & 2.696010 & -1.196190 \\
\hline
\end{tabular}




\begin{tabular}{|c|c|c|c|}
\hline $\mathrm{C}$ & 3.933580 & -0.229760 & 2.905240 \\
\hline $\mathrm{C}$ & 3.132700 & 1.043340 & 3.235770 \\
\hline $\mathrm{C}$ & 5.058290 & -0.452510 & 3.936480 \\
\hline $\mathrm{H}$ & 3.237350 & -1.069560 & 2.988720 \\
\hline $\mathrm{H}$ & 2.318410 & 1.181990 & 2.520520 \\
\hline $\mathrm{H}$ & 2.699330 & 0.959000 & 4.240480 \\
\hline $\mathrm{H}$ & 3.765040 & 1.939240 & 3.230120 \\
\hline $\mathrm{H}$ & 5.599110 & -1.387370 & 3.750800 \\
\hline $\mathrm{H}$ & 5.789450 & 0.364480 & 3.905710 \\
\hline $\mathrm{H}$ & 4.646670 & -0.495490 & 4.952370 \\
\hline $\mathrm{C}$ & 4.672280 & -2.355180 & -1.740880 \\
\hline $\mathrm{C}$ & 6.033590 & -2.984430 & -2.097230 \\
\hline $\mathrm{C}$ & 3.921210 & -1.901970 & -3.007210 \\
\hline $\mathrm{H}$ & 4.071020 & -3.142870 & -1.278340 \\
\hline $\mathrm{H}$ & 6.549810 & -3.346050 & -1.200810 \\
\hline $\mathrm{H}$ & 5.896870 & -3.833880 & -2.777610 \\
\hline $\mathrm{H}$ & 6.694190 & -2.263780 & -2.593600 \\
\hline $\mathrm{H}$ & 2.932110 & -1.509340 & -2.752240 \\
\hline $\mathrm{H}$ & 4.471530 & -1.121190 & -3.546030 \\
\hline $\mathrm{H}$ & 3.787370 & -2.746650 & -3.694120 \\
\hline $\mathrm{C}$ & -3.466390 & -2.781810 & 2.403490 \\
\hline $\mathrm{C}$ & -4.523760 & -3.687870 & 3.065200 \\
\hline $\mathrm{C}$ & -3.079210 & -1.611820 & 3.327780 \\
\hline $\mathrm{H}$ & -2.568950 & -3.390550 & 2.265630 \\
\hline $\mathrm{H}$ & -4.766750 & -4.544910 & 2.426950 \\
\hline $\mathrm{H}$ & -4.153890 & -4.072060 & 4.023680 \\
\hline $\mathrm{H}$ & -5.455260 & -3.143801 & 3.261570 \\
\hline $\mathrm{H}$ & -2.294550 & -0.999040 & 2.873690 \\
\hline $\mathrm{H}$ & -3.938280 & -0.964800 & 3.543060 \\
\hline $\mathrm{H}$ & -2.704110 & -1.992590 & 4.285640 \\
\hline $\mathrm{C}$ & -3.111050 & -2.544000 & -2.742830 \\
\hline $\mathrm{C}$ & -2.766660 & -1.293430 & -3.574110 \\
\hline $\mathrm{C}$ & -3.981110 & -3.515420 & -3.565410 \\
\hline $\mathrm{H}$ & -2.165480 & -3.049510 & -2.525840 \\
\hline $\mathrm{H}$ & -2.133480 & -0.607560 & -3.005180 \\
\hline $\mathrm{H}$ & -2.224960 & -1.585410 & -4.482250 \\
\hline $\mathrm{H}$ & -3.666100 & -0.751620 & -3.890380 \\
\hline $\mathrm{H}$ & -4.192540 & -4.436540 & -3.011480 \\
\hline $\mathrm{H}$ & -4.943190 & -3.059191 & -3.828730 \\
\hline $\mathrm{H}$ & -3.474610 & -3.790470 & -4.498610 \\
\hline $\mathrm{C}$ & -6.869550 & -0.199331 & -0.278100 \\
\hline $\mathrm{C}$ & -8.040350 & -1.030871 & -0.839430 \\
\hline $\mathrm{C}$ & -6.725200 & 1.127579 & -1.043960 \\
\hline $\mathrm{H}$ & -7.115030 & 0.049089 & 0.763840 \\
\hline $\mathrm{H}$ & -8.178330 & -1.955521 & -0.268440 \\
\hline $\mathrm{H}$ & -8.976550 & -0.460611 & -0.800340 \\
\hline $\mathrm{H}$ & -7.859900 & -1.308551 & -1.884860 \\
\hline $\mathrm{H}$ & -5.921630 & 1.744479 & -0.628520 \\
\hline $\mathrm{H}$ & -6.502580 & 0.958739 & -2.104180 \\
\hline $\mathrm{H}$ & -7.657850 & 1.702319 & -0.991760 \\
\hline $\mathrm{C}$ & -2.092420 & 6.110360 & 2.572510 \\
\hline $\mathrm{C}$ & -2.508410 & 7.163640 & 2.143840 \\
\hline $\mathrm{H}$ & -2.865551 & 8.096830 & 1.770770 \\
\hline
\end{tabular}




\section{1a allylboration TS 8}

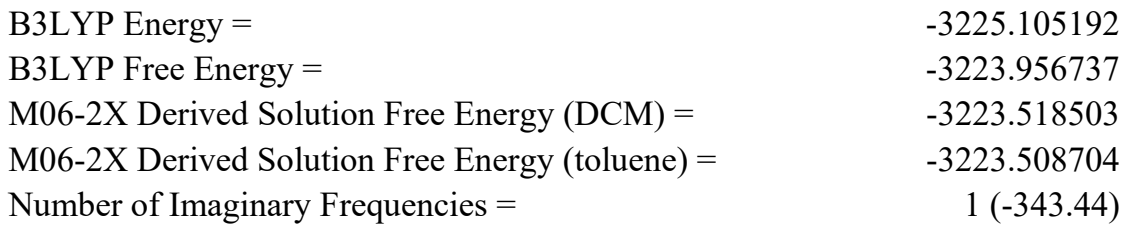

B3LYP/6-31G(d) Geometry

\begin{tabular}{|c|c|c|c|}
\hline B & -1.204981 & 2.996319 & -1.388890 \\
\hline $\mathrm{C}$ & -2.722481 & 2.132069 & -1.335780 \\
\hline $\mathrm{C}$ & -3.697341 & 3.164418 & -1.149270 \\
\hline $\mathrm{C}$ & -3.939912 & 3.753928 & 0.077150 \\
\hline $\mathrm{H}$ & -2.774170 & 1.621349 & -2.298390 \\
\hline $\mathrm{H}$ & -2.633210 & 1.425799 & -0.507650 \\
\hline $\mathrm{H}$ & -4.067291 & 3.671578 & -2.041140 \\
\hline $\mathrm{H}$ & -4.645092 & 4.575858 & 0.163480 \\
\hline $\mathrm{H}$ & -3.747591 & 3.193998 & 0.988450 \\
\hline $\mathrm{O}$ & -1.129262 & 3.867209 & -2.519740 \\
\hline $\mathrm{C}$ & 0.233068 & 3.846750 & -2.989580 \\
\hline $\mathrm{O}$ & -0.072041 & 2.091110 & -1.448400 \\
\hline $\mathrm{C}$ & 0.683549 & 2.371920 & -2.661450 \\
\hline $\mathrm{C}$ & 1.042048 & 4.902200 & -2.214780 \\
\hline $\mathrm{H}$ & 1.077558 & 4.676590 & -1.145350 \\
\hline $\mathrm{H}$ & 0.560017 & 5.877420 & -2.342450 \\
\hline $\mathrm{H}$ & 2.069268 & 4.979511 & -2.587260 \\
\hline $\mathrm{C}$ & 0.229638 & 4.196940 & -4.478270 \\
\hline $\mathrm{H}$ & -0.106582 & 5.231040 & -4.609280 \\
\hline $\mathrm{H}$ & -0.448001 & 3.550690 & -5.040760 \\
\hline $\mathrm{H}$ & 1.234578 & 4.112221 & -4.908060 \\
\hline $\mathrm{C}$ & 2.168429 & 2.199431 & -2.343170 \\
\hline $\mathrm{H}$ & 2.465029 & 2.797561 & -1.478970 \\
\hline $\mathrm{H}$ & 2.385650 & 1.151651 & -2.111160 \\
\hline $\mathrm{H}$ & 2.787849 & 2.486521 & -3.201060 \\
\hline $\mathrm{C}$ & 0.255440 & 1.366260 & -3.740200 \\
\hline $\mathrm{H}$ & -0.799860 & 1.488420 & -4.001850 \\
\hline $\mathrm{H}$ & 0.400650 & 0.347370 & -3.368850 \\
\hline $\mathrm{H}$ & 0.851950 & 1.482360 & -4.651390 \\
\hline $\mathrm{O}$ & -1.202022 & 3.709119 & -0.061020 \\
\hline $\mathrm{C}$ & -2.019262 & 4.695749 & 0.133430 \\
\hline $\mathrm{H}$ & -2.205342 & 5.352459 & -0.719480 \\
\hline $\mathrm{C}$ & -2.102432 & 5.348079 & 1.456460 \\
\hline $\mathrm{C}$ & -2.572653 & 6.670489 & 1.477840 \\
\hline $\mathrm{C}$ & -1.735062 & 4.722399 & 2.678500 \\
\hline $\mathrm{C}$ & -2.680443 & 7.384569 & 2.666260 \\
\hline $\mathrm{C}$ & -1.850332 & 5.462489 & 3.871370 \\
\hline $\mathrm{C}$ & -2.315783 & 6.773449 & 3.868990 \\
\hline $\mathrm{H}$ & -2.850163 & 7.142408 & 0.537920 \\
\hline $\mathrm{H}$ & -3.040784 & 8.409008 & 2.655300 \\
\hline $\mathrm{H}$ & -1.565202 & 4.982369 & 4.801960 \\
\hline $\mathrm{H}$ & -2.392633 & 7.319059 & 4.805300 \\
\hline
\end{tabular}




\begin{tabular}{|c|c|c|c|}
\hline $\mathrm{H}$ & 5.362812 & -3.148987 & 2.106950 \\
\hline $\mathrm{C}$ & 4.347192 & -3.372368 & 1.794400 \\
\hline $\mathrm{C}$ & 3.650882 & -2.413748 & 1.045090 \\
\hline $\mathrm{C}$ & 3.765413 & -4.594458 & 2.128340 \\
\hline $\mathrm{C}$ & 2.346332 & -2.735649 & 0.639800 \\
\hline $\mathrm{C}$ & 2.483373 & -4.896699 & 1.683200 \\
\hline $\mathrm{H}$ & 4.316813 & -5.314878 & 2.725910 \\
\hline $\mathrm{C}$ & 1.751372 & -3.980219 & 0.909520 \\
\hline $\mathrm{O}$ & 1.652731 & -1.826389 & -0.160780 \\
\hline $\mathrm{H}$ & 2.029033 & -5.849319 & 1.937800 \\
\hline $\mathrm{C}$ & 0.434753 & -4.378650 & 0.346460 \\
\hline $\mathrm{P}$ & 0.361701 & -1.056040 & 0.467510 \\
\hline $\mathrm{C}$ & -0.688558 & -3.530980 & 0.320540 \\
\hline $\mathrm{C}$ & 0.278213 & -5.680220 & -0.159820 \\
\hline $\mathrm{O}$ & -0.621368 & -2.270030 & 0.899540 \\
\hline $\mathrm{O}$ & 0.621640 & -0.177420 & 1.628440 \\
\hline $\mathrm{O}$ & -0.283859 & -0.427660 & -0.825890 \\
\hline $\mathrm{C}$ & -1.946138 & -3.960491 & -0.135970 \\
\hline $\mathrm{C}$ & -0.950387 & -6.128751 & -0.630760 \\
\hline $\mathrm{H}$ & 1.142314 & -6.336899 & -0.186200 \\
\hline $\mathrm{H}$ & -0.157760 & 0.574030 & -0.970160 \\
\hline $\mathrm{C}$ & -2.052657 & -5.277221 & -0.605230 \\
\hline $\mathrm{H}$ & -1.050046 & -7.140271 & -1.014020 \\
\hline $\mathrm{H}$ & -3.020797 & -5.626522 & -0.952200 \\
\hline $\mathrm{C}$ & 4.337361 & -1.137778 & 0.646980 \\
\hline $\mathrm{C}$ & 4.921331 & -1.036138 & -0.638780 \\
\hline $\mathrm{C}$ & 4.478310 & -0.079038 & 1.572730 \\
\hline $\mathrm{C}$ & 5.640010 & 0.118013 & -0.966750 \\
\hline $\mathrm{C}$ & 5.218330 & 1.048133 & 1.194190 \\
\hline $\mathrm{C}$ & 5.811130 & 1.170243 & -0.062900 \\
\hline $\mathrm{H}$ & 6.092030 & 0.187713 & -1.953440 \\
\hline $\mathrm{H}$ & 5.339559 & 1.860753 & 1.906300 \\
\hline $\mathrm{C}$ & -3.163708 & -3.082382 & -0.111640 \\
\hline $\mathrm{C}$ & -3.707088 & -2.598452 & -1.326640 \\
\hline $\mathrm{C}$ & -3.815148 & -2.800152 & 1.111190 \\
\hline $\mathrm{C}$ & -4.893839 & -1.858173 & -1.290590 \\
\hline $\mathrm{C}$ & -4.994859 & -2.047233 & 1.088390 \\
\hline $\mathrm{C}$ & -5.559049 & -1.571843 & -0.095880 \\
\hline $\mathrm{H}$ & -5.311129 & -1.497373 & -2.227630 \\
\hline $\mathrm{H}$ & -5.499999 & -1.832503 & 2.027870 \\
\hline $\mathrm{C}$ & 6.639689 & 2.399313 & -0.414590 \\
\hline $\mathrm{C}$ & 6.090349 & 3.147423 & -1.642930 \\
\hline $\mathrm{C}$ & 8.126269 & 2.042254 & -0.607930 \\
\hline $\mathrm{H}$ & 6.576329 & 3.084903 & 0.441620 \\
\hline $\mathrm{H}$ & 5.044279 & 3.436212 & -1.495190 \\
\hline $\mathrm{H}$ & 6.672888 & 4.056923 & -1.834160 \\
\hline $\mathrm{H}$ & 6.141299 & 2.525993 & -2.545020 \\
\hline $\mathrm{H}$ & 8.531590 & 1.543484 & 0.279210 \\
\hline $\mathrm{H}$ & 8.264230 & 1.367904 & -1.461630 \\
\hline $\mathrm{H}$ & 8.721519 & 2.944314 & -0.795350 \\
\hline $\mathrm{C}$ & 3.883190 & -0.138438 & 2.979660 \\
\hline $\mathrm{C}$ & 3.128290 & 1.147102 & 3.363590 \\
\hline $\mathrm{C}$ & 4.970761 & -0.458168 & 4.025930 \\
\hline
\end{tabular}




\begin{tabular}{|c|c|c|c|}
\hline $\mathrm{H}$ & 3.149981 & -0.950138 & 2.998900 \\
\hline $\mathrm{H}$ & 2.361010 & 1.384151 & 2.623160 \\
\hline $\mathrm{H}$ & 2.633370 & 1.009611 & 4.332430 \\
\hline $\mathrm{H}$ & 3.801809 & 2.006892 & 3.462800 \\
\hline $\mathrm{H}$ & 5.471481 & -1.409267 & 3.814330 \\
\hline $\mathrm{H}$ & 5.738780 & 0.324903 & 4.045160 \\
\hline $\mathrm{H}$ & 4.532081 & -0.523338 & 5.029180 \\
\hline $\mathrm{C}$ & 4.825081 & -2.157638 & -1.673820 \\
\hline $\mathrm{C}$ & 6.214982 & -2.716367 & -2.036410 \\
\hline $\mathrm{C}$ & 4.058871 & -1.713878 & -2.934470 \\
\hline $\mathrm{H}$ & 4.258932 & -2.982038 & -1.231080 \\
\hline $\mathrm{H}$ & 6.743382 & -3.071907 & -1.144740 \\
\hline $\mathrm{H}$ & 6.119502 & -3.557387 & -2.733990 \\
\hline $\mathrm{H}$ & 6.844641 & -1.957387 & -2.515470 \\
\hline $\mathrm{H}$ & 3.047081 & -1.383049 & -2.678900 \\
\hline $\mathrm{H}$ & 4.567921 & -0.889118 & -3.447510 \\
\hline $\mathrm{H}$ & 3.975872 & -2.545908 & -3.644510 \\
\hline $\mathrm{C}$ & -3.307298 & -3.318092 & 2.456240 \\
\hline $\mathrm{C}$ & -4.302077 & -4.314142 & 3.084840 \\
\hline $\mathrm{C}$ & -2.980019 & -2.170342 & 3.430320 \\
\hline $\mathrm{H}$ & -2.377638 & -3.866701 & 2.283010 \\
\hline $\mathrm{H}$ & -4.506697 & -5.151382 & 2.407960 \\
\hline $\mathrm{H}$ & -3.896237 & -4.722882 & 4.018280 \\
\hline $\mathrm{H}$ & -5.259218 & -3.834073 & 3.320650 \\
\hline $\mathrm{H}$ & -2.234969 & -1.493671 & 3.001700 \\
\hline $\mathrm{H}$ & -3.872929 & -1.584032 & 3.678770 \\
\hline $\mathrm{H}$ & -2.576398 & -2.571421 & 4.367970 \\
\hline $\mathrm{C}$ & -3.046988 & -2.852692 & -2.682960 \\
\hline $\mathrm{C}$ & -2.687939 & -1.538521 & -3.401520 \\
\hline $\mathrm{C}$ & -3.919858 & -3.750362 & -3.581530 \\
\hline $\mathrm{H}$ & -2.106998 & -3.383671 & -2.508710 \\
\hline $\mathrm{H}$ & -2.034819 & -0.924141 & -2.775350 \\
\hline $\mathrm{H}$ & -2.163869 & -1.750261 & -4.341680 \\
\hline $\mathrm{H}$ & -3.582359 & -0.953152 & -3.646720 \\
\hline $\mathrm{H}$ & -4.138057 & -4.710172 & -3.099660 \\
\hline $\mathrm{H}$ & -4.878798 & -3.272323 & -3.814820 \\
\hline $\mathrm{H}$ & -3.410198 & -3.955872 & -4.530760 \\
\hline $\mathrm{C}$ & -6.873229 & -0.801164 & -0.074180 \\
\hline $\mathrm{C}$ & -8.018909 & -1.633354 & -0.683170 \\
\hline $\mathrm{C}$ & -6.768960 & 0.572696 & -0.759890 \\
\hline $\mathrm{H}$ & -7.122749 & -0.622824 & 0.980930 \\
\hline $\mathrm{H}$ & -8.128488 & -2.593034 & -0.166360 \\
\hline $\mathrm{H}$ & -8.971889 & -1.094875 & -0.611850 \\
\hline $\mathrm{H}$ & -7.831959 & -1.845014 & -1.742850 \\
\hline $\mathrm{H}$ & -5.972070 & 1.180097 & -0.318010 \\
\hline $\mathrm{H}$ & -6.555780 & 0.471237 & -1.830670 \\
\hline $\mathrm{H}$ & -7.712900 & 1.122776 & -0.662860 \\
\hline $\mathrm{C}$ & -1.256991 & 3.381749 & 2.743340 \\
\hline $\mathrm{C}$ & -0.839521 & 2.248669 & 2.836140 \\
\hline $\mathrm{H}$ & -0.418000 & 1.262530 & 2.785610 \\
\hline
\end{tabular}

1d allylboration TS 7 (TS-4Si-A)

B3LYP Energy =

$-3456.180026$ 
B3LYP Free Energy =

$-3454.953444$

M06-2X Derived Solution Free Energy $(\mathrm{DCM})=$

$-3454.470228$

M06-2X Derived Solution Free Energy (toluene) =

$-3454.460738$

Number of Imaginary Frequencies $=$

$1(-339.55)$

B3LYP/6-31G(d) Geometry

\begin{tabular}{|c|c|c|c|}
\hline B & 2.303570 & -0.412328 & -1.676070 \\
\hline $\mathrm{C}$ & 2.999699 & 1.183472 & -1.850880 \\
\hline $\mathrm{C}$ & 4.248389 & 1.081693 & -1.155580 \\
\hline $\mathrm{C}$ & 4.352689 & 1.144353 & 0.221040 \\
\hline $\mathrm{H}$ & 3.083359 & 1.319482 & -2.930310 \\
\hline $\mathrm{H}$ & 2.278449 & 1.875062 & -1.409680 \\
\hline $\mathrm{H}$ & 5.097889 & 0.682594 & -1.711240 \\
\hline $\mathrm{H}$ & 5.310899 & 0.992584 & 0.708570 \\
\hline $\mathrm{H}$ & 3.604959 & 1.688613 & 0.791030 \\
\hline $\mathrm{O}$ & 3.081371 & -1.399618 & -2.356820 \\
\hline $\mathrm{C}$ & 2.164921 & -2.355208 & -2.927500 \\
\hline $\mathrm{O}$ & 0.954070 & -0.474789 & -2.205060 \\
\hline $\mathrm{C}$ & 0.915581 & -1.460129 & -3.273170 \\
\hline $\mathrm{C}$ & 1.848352 & -3.430158 & -1.872750 \\
\hline $\mathrm{H}$ & 1.333222 & -3.004989 & -1.006790 \\
\hline $\mathrm{H}$ & 2.789672 & -3.871188 & -1.528110 \\
\hline $\mathrm{H}$ & 1.227493 & -4.233199 & -2.285600 \\
\hline $\mathrm{C}$ & 2.842412 & -3.001938 & -4.136190 \\
\hline $\mathrm{H}$ & 3.706312 & -3.585607 & -3.800820 \\
\hline $\mathrm{H}$ & 3.198831 & -2.250368 & -4.844350 \\
\hline $\mathrm{H}$ & 2.158622 & -3.681538 & -4.658170 \\
\hline $\mathrm{C}$ & -0.433189 & -2.178200 & -3.226650 \\
\hline $\mathrm{H}$ & -0.636398 & -2.601470 & -2.240310 \\
\hline $\mathrm{H}$ & -1.239069 & -1.475620 & -3.463990 \\
\hline $\mathrm{H}$ & -0.466068 & -2.986700 & -3.966140 \\
\hline $\mathrm{C}$ & 1.063710 & -0.708709 & -4.604360 \\
\hline $\mathrm{H}$ & 2.037630 & -0.215688 & -4.676560 \\
\hline $\mathrm{H}$ & 0.286130 & 0.059021 & -4.670500 \\
\hline $\mathrm{H}$ & 0.952891 & -1.381839 & -5.461480 \\
\hline $\mathrm{O}$ & 2.245770 & -0.569088 & -0.184740 \\
\hline $\mathrm{C}$ & 3.346740 & -0.735058 & 0.484920 \\
\hline $\mathrm{H}$ & 4.135151 & -1.320877 & 0.013780 \\
\hline $\mathrm{C}$ & 3.259150 & -0.784868 & 1.955700 \\
\hline $\mathrm{C}$ & 4.392401 & -1.163497 & 2.725970 \\
\hline $\mathrm{C}$ & 2.045520 & -0.495908 & 2.595440 \\
\hline $\mathrm{C}$ & 4.271881 & -1.223127 & 4.127200 \\
\hline $\mathrm{C}$ & 1.949360 & -0.565658 & 3.983160 \\
\hline $\mathrm{C}$ & 3.063510 & -0.923408 & 4.748620 \\
\hline $\mathrm{H}$ & 5.138151 & -1.511606 & 4.714180 \\
\hline $\mathrm{H}$ & 0.999960 & -0.349179 & 4.463820 \\
\hline $\mathrm{H}$ & 2.988920 & -0.977708 & 5.831280 \\
\hline $\mathrm{H}$ & -6.120119 & -2.364784 & 2.473180 \\
\hline $\mathrm{C}$ & -5.732999 & -1.408593 & 2.133620 \\
\hline $\mathrm{C}$ & -4.672969 & -1.401703 & 1.216400 \\
\hline $\mathrm{C}$ & -6.291510 & -0.220754 & 2.604220 \\
\hline $\mathrm{C}$ & -4.201440 & -0.151792 & 0.785170 \\
\hline $\mathrm{C}$ & -5.813851 & 1.001747 & 2.143920 \\
\hline
\end{tabular}




\begin{tabular}{|c|c|c|c|}
\hline $\mathrm{H}$ & -7.102770 & -0.249534 & 3.326180 \\
\hline $\mathrm{C}$ & -4.766501 & 1.062857 & 1.209860 \\
\hline $\mathrm{O}$ & -3.215490 & -0.119952 & -0.197280 \\
\hline $\mathrm{H}$ & -6.243521 & 1.928456 & 2.512150 \\
\hline $\mathrm{C}$ & -4.335312 & 2.374688 & 0.662170 \\
\hline$P$ & -1.712500 & 0.392669 & 0.161560 \\
\hline $\mathrm{C}$ & -2.983472 & 2.720438 & 0.476150 \\
\hline $\mathrm{C}$ & -5.304652 & 3.341037 & 0.345870 \\
\hline $\mathrm{O}$ & -1.987961 & 1.832029 & 0.866890 \\
\hline $\mathrm{O}$ & -0.906280 & -0.462030 & 1.057740 \\
\hline $\mathrm{O}$ & -1.174241 & 0.692940 & -1.287860 \\
\hline $\mathrm{C}$ & -2.584643 & 3.993849 & 0.038430 \\
\hline $\mathrm{C}$ & -4.940123 & 4.605197 & -0.103500 \\
\hline $\mathrm{H}$ & -6.353962 & 3.081696 & 0.448950 \\
\hline $\mathrm{H}$ & -0.333680 & 0.194580 & -1.566700 \\
\hline $\mathrm{C}$ & -3.591693 & 4.927948 & -0.243250 \\
\hline $\mathrm{H}$ & -5.704404 & 5.339827 & -0.340700 \\
\hline $\mathrm{H}$ & -3.299844 & 5.921998 & -0.568860 \\
\hline $\mathrm{C}$ & -4.109148 & -2.693542 & 0.698640 \\
\hline $\mathrm{C}$ & -4.467868 & -3.150363 & -0.592640 \\
\hline $\mathrm{C}$ & -3.266018 & -3.480042 & 1.516620 \\
\hline $\mathrm{C}$ & -3.975407 & -4.381582 & -1.035630 \\
\hline $\mathrm{C}$ & -2.809247 & -4.709221 & 1.024520 \\
\hline $\mathrm{C}$ & -3.146497 & -5.181322 & -0.243680 \\
\hline $\mathrm{H}$ & -4.256027 & -4.729632 & -2.027060 \\
\hline $\mathrm{H}$ & -2.164027 & -5.320401 & 1.651440 \\
\hline $\mathrm{C}$ & -1.144593 & 4.403680 & -0.087080 \\
\hline $\mathrm{C}$ & -0.560403 & 4.561410 & -1.367660 \\
\hline $\mathrm{C}$ & -0.399353 & 4.726840 & 1.069830 \\
\hline $\mathrm{C}$ & 0.738147 & 5.075151 & -1.460060 \\
\hline $\mathrm{C}$ & 0.899716 & 5.226481 & 0.918660 \\
\hline $\mathrm{C}$ & 1.485316 & 5.426011 & -0.331970 \\
\hline $\mathrm{H}$ & 1.173436 & 5.213861 & -2.446430 \\
\hline $\mathrm{H}$ & 1.469526 & 5.488691 & 1.807830 \\
\hline $\mathrm{C}$ & -2.628736 & -6.526801 & -0.735800 \\
\hline $\mathrm{C}$ & -1.740666 & -6.380241 & -1.985670 \\
\hline $\mathrm{C}$ & -3.773545 & -7.526332 & -0.986690 \\
\hline $\mathrm{H}$ & -2.001176 & -6.942121 & 0.064780 \\
\hline $\mathrm{H}$ & -0.902596 & -5.700440 & -1.795800 \\
\hline $\mathrm{H}$ & -1.331475 & -7.352171 & -2.287410 \\
\hline $\mathrm{H}$ & -2.309516 & -5.980591 & -2.833710 \\
\hline $\mathrm{H}$ & -4.384915 & -7.660462 & -0.087620 \\
\hline $\mathrm{H}$ & -4.434776 & -7.181893 & -1.790850 \\
\hline $\mathrm{H}$ & -3.375775 & -8.505652 & -1.279520 \\
\hline $\mathrm{C}$ & -2.849638 & -3.050842 & 2.923710 \\
\hline $\mathrm{C}$ & -1.323718 & -3.097451 & 3.127290 \\
\hline $\mathrm{C}$ & -3.570608 & -3.891932 & 3.996500 \\
\hline $\mathrm{H}$ & -3.154309 & -2.009162 & 3.061580 \\
\hline $\mathrm{H}$ & -0.817029 & -2.477800 & 2.383680 \\
\hline $\mathrm{H}$ & -1.070088 & -2.715700 & 4.124300 \\
\hline $\mathrm{H}$ & -0.933038 & -4.120000 & 3.062770 \\
\hline $\mathrm{H}$ & -4.660198 & -3.814633 & 3.907890 \\
\hline $\mathrm{H}$ & -3.306057 & -4.952892 & 3.910640 \\
\hline
\end{tabular}




\begin{tabular}{|c|c|c|c|}
\hline $\mathrm{H}$ & -3.288248 & -3.556942 & 5.002200 \\
\hline $\mathrm{C}$ & -5.402179 & -2.362973 & -1.511600 \\
\hline $\mathrm{C}$ & -6.704158 & -3.139534 & -1.790670 \\
\hline $\mathrm{C}$ & -4.708529 & -1.951663 & -2.824220 \\
\hline $\mathrm{H}$ & -5.688119 & -1.440793 & -0.998020 \\
\hline $\mathrm{H}$ & -7.217558 & -3.400754 & -0.858470 \\
\hline $\mathrm{H}$ & -7.388339 & -2.534994 & -2.398520 \\
\hline $\mathrm{H}$ & -6.509818 & -4.070674 & -2.335980 \\
\hline $\mathrm{H}$ & -3.818889 & -1.347812 & -2.620990 \\
\hline $\mathrm{H}$ & -4.401998 & -2.826873 & -3.409640 \\
\hline $\mathrm{H}$ & -5.389709 & -1.358343 & -3.446610 \\
\hline $\mathrm{C}$ & -0.967733 & 4.586910 & 2.481280 \\
\hline $\mathrm{C}$ & -1.092854 & 5.956510 & 3.177700 \\
\hline $\mathrm{C}$ & -0.147222 & 3.601830 & 3.335600 \\
\hline $\mathrm{H}$ & -1.978273 & 4.177169 & 2.403570 \\
\hline $\mathrm{H}$ & -1.713934 & 6.643709 & 2.592080 \\
\hline $\mathrm{H}$ & -1.551804 & 5.843049 & 4.167420 \\
\hline $\mathrm{H}$ & -0.113354 & 6.429190 & 3.317330 \\
\hline $\mathrm{H}$ & -0.095412 & 2.619020 & 2.857240 \\
\hline $\mathrm{H}$ & 0.877037 & 3.960201 & 3.494800 \\
\hline $\mathrm{H}$ & -0.610672 & 3.477750 & 4.322080 \\
\hline $\mathrm{C}$ & -1.305883 & 4.209979 & -2.656340 \\
\hline $\mathrm{C}$ & -0.494122 & 3.271510 & -3.569140 \\
\hline $\mathrm{C}$ & -1.726024 & 5.478219 & -3.426670 \\
\hline $\mathrm{H}$ & -2.218273 & 3.672559 & -2.381210 \\
\hline $\mathrm{H}$ & -0.203882 & 2.360970 & -3.038500 \\
\hline $\mathrm{H}$ & -1.097422 & 2.982080 & -4.438390 \\
\hline $\mathrm{H}$ & 0.414347 & 3.754201 & -3.948550 \\
\hline $\mathrm{H}$ & -2.358284 & 6.131799 & -2.815930 \\
\hline $\mathrm{H}$ & -0.849794 & 6.060400 & -3.737310 \\
\hline $\mathrm{H}$ & -2.288464 & 5.211939 & -4.329920 \\
\hline $\mathrm{C}$ & 2.870116 & 6.051262 & -0.449550 \\
\hline $\mathrm{C}$ & 2.786295 & 7.476422 & -1.031380 \\
\hline $\mathrm{C}$ & 3.852366 & 5.186653 & -1.259300 \\
\hline $\mathrm{H}$ & 3.271086 & 6.136872 & 0.569930 \\
\hline $\mathrm{H}$ & 2.122975 & 8.109922 & -0.432380 \\
\hline $\mathrm{H}$ & 3.777505 & 7.945483 & -1.056620 \\
\hline $\mathrm{H}$ & 2.396365 & 7.460672 & -2.056170 \\
\hline $\mathrm{H}$ & 3.955567 & 4.186003 & -0.826780 \\
\hline $\mathrm{H}$ & 3.520887 & 5.068503 & -2.297640 \\
\hline $\mathrm{H}$ & 4.845066 & 5.652443 & -1.281210 \\
\hline $\mathrm{C}$ & 5.639351 & -1.479986 & 2.115030 \\
\hline $\mathrm{C}$ & 6.709891 & -1.757585 & 1.605640 \\
\hline $\mathrm{H}$ & 1.172690 & -0.254519 & 1.995830 \\
\hline $\mathrm{C}$ & 7.963711 & -2.085965 & 1.013270 \\
\hline $\mathrm{C}$ & 8.087241 & -2.205074 & -0.385980 \\
\hline $\mathrm{C}$ & 9.100881 & -2.297804 & 1.818830 \\
\hline $\mathrm{C}$ & 9.315161 & -2.525304 & -0.957970 \\
\hline $\mathrm{H}$ & 7.213311 & -2.048385 & -1.011170 \\
\hline $\mathrm{C}$ & 10.324392 & -2.617483 & 1.237340 \\
\hline $\mathrm{H}$ & 9.009641 & -2.207964 & 2.896880 \\
\hline $\mathrm{C}$ & 10.436702 & -2.732033 & -0.150660 \\
\hline $\mathrm{H}$ & 9.397242 & -2.615234 & -2.037680 \\
\hline
\end{tabular}




\begin{tabular}{|c|c|c|c|}
\hline $\mathrm{H}$ & 11.193682 & -2.778212 & 1.869110 \\
\hline $\mathrm{H}$ & 11.393332 & -2.981972 & -0.600990 \\
\hline
\end{tabular}

\section{1d allylboration TS 8}

B3LYP Energy =

$-3456.168971$

B3LYP Free Energy =

$-3454.941412$

M06-2X Derived Solution Free Energy $(\mathrm{DCM})=$

$-3454.462311$

M06-2X Derived Solution Free Energy (toluene) =

$-3454.452027$

Number of Imaginary Frequencies $=$

$1(-357.43)$

B3LYP/6-31G(d) Geometry

\begin{tabular}{|c|c|c|c|}
\hline B & -1.846419 & 2.365881 & -1.755500 \\
\hline $\mathrm{C}$ & -2.967820 & 1.043531 & -1.926980 \\
\hline $\mathrm{C}$ & -4.214610 & 1.696392 & -2.198480 \\
\hline $\mathrm{C}$ & -4.973259 & 2.317582 & -1.222370 \\
\hline $\mathrm{H}$ & -2.576010 & 0.450561 & -2.754130 \\
\hline $\mathrm{H}$ & -2.926780 & 0.494291 & -0.983860 \\
\hline $\mathrm{H}$ & -4.435059 & 1.936432 & -3.239230 \\
\hline $\mathrm{H}$ & -5.881719 & 2.848222 & -1.493220 \\
\hline $\mathrm{H}$ & -4.914319 & 1.973342 & -0.192900 \\
\hline $\mathrm{O}$ & -1.751429 & 3.145341 & -2.954170 \\
\hline $\mathrm{C}$ & -0.395419 & 3.623490 & -3.044190 \\
\hline $\mathrm{O}$ & -0.508899 & 1.910190 & -1.421710 \\
\hline $\mathrm{C}$ & 0.418601 & 2.425480 & -2.429010 \\
\hline $\mathrm{C}$ & -0.257918 & 4.920030 & -2.225440 \\
\hline $\mathrm{H}$ & -0.447878 & 4.749920 & -1.162480 \\
\hline $\mathrm{H}$ & -0.990058 & 5.647940 & -2.591710 \\
\hline $\mathrm{H}$ & 0.739362 & 5.360330 & -2.332720 \\
\hline $\mathrm{C}$ & -0.092049 & 3.918800 & -4.514050 \\
\hline $\mathrm{H}$ & -0.705208 & 4.761580 & -4.850930 \\
\hline $\mathrm{H}$ & -0.321459 & 3.061860 & -5.151160 \\
\hline $\mathrm{H}$ & 0.960551 & 4.190409 & -4.654770 \\
\hline $\mathrm{C}$ & 1.715541 & 2.812819 & -1.721180 \\
\hline $\mathrm{H}$ & 1.529011 & 3.485039 & -0.880670 \\
\hline $\mathrm{H}$ & 2.218361 & 1.922369 & -1.330540 \\
\hline $\mathrm{H}$ & 2.405981 & 3.302089 & -2.417670 \\
\hline $\mathrm{C}$ & 0.690270 & 1.319650 & -3.459960 \\
\hline $\mathrm{H}$ & -0.224670 & 1.036160 & -3.988960 \\
\hline $\mathrm{H}$ & 1.085400 & 0.431329 & -2.960790 \\
\hline $\mathrm{H}$ & 1.429140 & 1.646939 & -4.198890 \\
\hline $\mathrm{O}$ & -2.462199 & 3.147881 & -0.603720 \\
\hline $\mathrm{C}$ & -3.529259 & 3.828881 & -0.910210 \\
\hline $\mathrm{H}$ & -3.553289 & 4.229211 & -1.924650 \\
\hline $\mathrm{C}$ & -4.238728 & 4.670892 & 0.075120 \\
\hline $\mathrm{C}$ & -4.910408 & 5.784122 & -0.459240 \\
\hline $\mathrm{C}$ & -4.293958 & 4.439822 & 1.474650 \\
\hline $\mathrm{C}$ & -5.616087 & 6.670942 & 0.345020 \\
\hline $\mathrm{C}$ & -5.017938 & 5.351592 & 2.274640 \\
\hline $\mathrm{C}$ & -5.667678 & 6.449122 & 1.724040 \\
\hline $\mathrm{H}$ & -4.864678 & 5.953532 & -1.532400 \\
\hline $\mathrm{H}$ & -6.117467 & 7.527052 & -0.096590 \\
\hline $\mathrm{H}$ & -5.055288 & 5.172632 & 3.344290 \\
\hline
\end{tabular}




\begin{tabular}{|c|c|c|c|}
\hline $\mathrm{H}$ & -6.213367 & 7.131412 & 2.369850 \\
\hline $\mathrm{H}$ & 6.268499 & -1.298533 & 2.027340 \\
\hline $\mathrm{C}$ & 5.410879 & -1.854502 & 1.660700 \\
\hline $\mathrm{C}$ & 4.433959 & -1.165272 & 0.927120 \\
\hline $\mathrm{C}$ & 5.303598 & -3.220842 & 1.912880 \\
\hline $\mathrm{C}$ & 3.341109 & -1.907452 & 0.452030 \\
\hline $\mathrm{C}$ & 4.228508 & -3.935512 & 1.397660 \\
\hline $\mathrm{H}$ & 6.064048 & -3.728583 & 2.499380 \\
\hline $\mathrm{C}$ & 3.233468 & -3.297352 & 0.638710 \\
\hline $\mathrm{O}$ & 2.371789 & -1.265851 & -0.325100 \\
\hline $\mathrm{H}$ & 4.142178 & -5.001532 & 1.584440 \\
\hline $\mathrm{C}$ & 2.166758 & -4.109191 & -0.001600 \\
\hline $\mathrm{P}$ & 0.889479 & -1.058681 & 0.329680 \\
\hline $\mathrm{C}$ & 0.813518 & -3.725371 & -0.032990 \\
\hline $\mathrm{C}$ & 2.509698 & -5.340251 & -0.586340 \\
\hline $\mathrm{O}$ & 0.404099 & -2.575380 & 0.633990 \\
\hline $\mathrm{O}$ & 0.836610 & -0.226641 & 1.550140 \\
\hline $\mathrm{O}$ & 0.038699 & -0.602880 & -0.918110 \\
\hline $\mathrm{C}$ & -0.185512 & -4.540690 & -0.591980 \\
\hline $\mathrm{C}$ & 1.542837 & -6.167421 & -1.145590 \\
\hline $\mathrm{H}$ & 3.554187 & -5.635802 & -0.603810 \\
\hline $\mathrm{H}$ & -0.170050 & 0.390570 & -0.991910 \\
\hline $\mathrm{C}$ & 0.207587 & -5.770380 & -1.138300 \\
\hline $\mathrm{H}$ & 1.828477 & -7.117531 & -1.587980 \\
\hline $\mathrm{H}$ & -0.556363 & -6.415520 & -1.562470 \\
\hline $\mathrm{C}$ & 4.650570 & 0.291108 & 0.624930 \\
\hline $\mathrm{C}$ & 5.237220 & 0.660918 & -0.609560 \\
\hline $\mathrm{C}$ & 4.389390 & 1.275228 & 1.605680 \\
\hline $\mathrm{C}$ & 5.564731 & 2.003068 & -0.828760 \\
\hline $\mathrm{C}$ & 4.746761 & 2.602318 & 1.335400 \\
\hline $\mathrm{C}$ & 5.341891 & 2.990878 & 0.134600 \\
\hline $\mathrm{H}$ & 6.024921 & 2.278887 & -1.774770 \\
\hline $\mathrm{H}$ & 4.562251 & 3.360398 & 2.092640 \\
\hline $\mathrm{C}$ & -1.635492 & -4.153060 & -0.609050 \\
\hline $\mathrm{C}$ & -2.243682 & -3.758099 & -1.825300 \\
\hline $\mathrm{C}$ & -2.411802 & -4.250929 & 0.567510 \\
\hline $\mathrm{C}$ & -3.610742 & -3.461449 & -1.831480 \\
\hline $\mathrm{C}$ & -3.775022 & -3.940509 & 0.503890 \\
\hline $\mathrm{C}$ & -4.397822 & -3.543458 & -0.679770 \\
\hline $\mathrm{H}$ & -4.074182 & -3.161448 & -2.768360 \\
\hline $\mathrm{H}$ & -4.375352 & -4.020038 & 1.407660 \\
\hline $\mathrm{C}$ & 5.759292 & 4.438667 & -0.093480 \\
\hline $\mathrm{C}$ & 5.051482 & 5.072018 & -1.305170 \\
\hline $\mathrm{C}$ & 7.289382 & 4.571587 & -0.219710 \\
\hline $\mathrm{H}$ & 5.453372 & 5.004808 & 0.796970 \\
\hline $\mathrm{H}$ & 3.962802 & 5.026558 & -1.195280 \\
\hline $\mathrm{H}$ & 5.340312 & 6.124408 & -1.414800 \\
\hline $\mathrm{H}$ & 5.316252 & 4.557138 & -2.236560 \\
\hline $\mathrm{H}$ & 7.796071 & 4.157797 & 0.659000 \\
\hline $\mathrm{H}$ & 7.662611 & 4.037637 & -1.101860 \\
\hline $\mathrm{H}$ & 7.581062 & 5.624317 & -0.318560 \\
\hline $\mathrm{C}$ & 3.771100 & 0.933398 & 2.961510 \\
\hline $\mathrm{C}$ & 2.615751 & 1.876069 & 3.343900 \\
\hline
\end{tabular}




\begin{tabular}{|c|c|c|c|}
\hline $\mathrm{C}$ & 4.842790 & 0.914568 & 4.070730 \\
\hline $\mathrm{H}$ & 3.344070 & -0.071032 & 2.890030 \\
\hline $\mathrm{H}$ & 1.852681 & 1.891639 & 2.562690 \\
\hline $\mathrm{H}$ & 2.140800 & 1.525199 & 4.267440 \\
\hline $\mathrm{H}$ & 2.962281 & 2.900859 & 3.524240 \\
\hline $\mathrm{H}$ & 5.637060 & 0.191097 & 3.856520 \\
\hline $\mathrm{H}$ & 5.311491 & 1.900078 & 4.182840 \\
\hline $\mathrm{H}$ & 4.392760 & 0.645768 & 5.034270 \\
\hline $\mathrm{C}$ & 5.564150 & -0.360392 & -1.699930 \\
\hline $\mathrm{C}$ & 7.082650 & -0.457813 & -1.945400 \\
\hline $\mathrm{C}$ & 4.808550 & -0.067612 & -3.009770 \\
\hline $\mathrm{H}$ & 5.234559 & -1.344662 & -1.355720 \\
\hline $\mathrm{H}$ & 7.617109 & -0.713243 & -1.023530 \\
\hline $\mathrm{H}$ & 7.302459 & -1.231343 & -2.691340 \\
\hline $\mathrm{H}$ & 7.492660 & 0.488967 & -2.316350 \\
\hline $\mathrm{H}$ & 3.726600 & -0.060342 & -2.843350 \\
\hline $\mathrm{H}$ & 5.093970 & 0.902868 & -3.432930 \\
\hline $\mathrm{H}$ & 5.032529 & -0.835532 & -3.760270 \\
\hline $\mathrm{C}$ & -1.830262 & -4.735129 & 1.894530 \\
\hline $\mathrm{C}$ & -2.399053 & -6.116949 & 2.275480 \\
\hline $\mathrm{C}$ & -2.037142 & -3.717219 & 3.030170 \\
\hline $\mathrm{H}$ & -0.751162 & -4.859410 & 1.768290 \\
\hline $\mathrm{H}$ & -2.208523 & -6.855559 & 1.488560 \\
\hline $\mathrm{H}$ & -1.938103 & -6.480389 & 3.202120 \\
\hline $\mathrm{H}$ & -3.482923 & -6.073199 & 2.436470 \\
\hline $\mathrm{H}$ & -1.606791 & -2.745690 & 2.771730 \\
\hline $\mathrm{H}$ & -3.099642 & -3.567139 & 3.255380 \\
\hline $\mathrm{H}$ & -1.553582 & -4.071010 & 3.949020 \\
\hline $\mathrm{C}$ & -1.467422 & -3.661950 & -3.140120 \\
\hline $\mathrm{C}$ & -1.540301 & -2.254390 & -3.759360 \\
\hline $\mathrm{C}$ & -1.935162 & -4.730449 & -4.148100 \\
\hline $\mathrm{H}$ & -0.412752 & -3.856910 & -2.926910 \\
\hline $\mathrm{H}$ & -1.155771 & -1.507950 & -3.058830 \\
\hline $\mathrm{H}$ & -0.937231 & -2.211020 & -4.674720 \\
\hline $\mathrm{H}$ & -2.568021 & -1.981429 & -4.028020 \\
\hline $\mathrm{H}$ & -1.834743 & -5.741319 & -3.736990 \\
\hline $\mathrm{H}$ & -2.987592 & -4.588349 & -4.421590 \\
\hline $\mathrm{H}$ & -1.340722 & -4.677860 & -5.068400 \\
\hline $\mathrm{C}$ & -5.888942 & -3.232308 & -0.707050 \\
\hline $\mathrm{C}$ & -6.662212 & -4.225387 & -1.595580 \\
\hline $\mathrm{C}$ & -6.170351 & -1.778828 & -1.130640 \\
\hline $\mathrm{H}$ & -6.260712 & -3.352438 & 0.319780 \\
\hline $\mathrm{H}$ & -6.499482 & -5.258157 & -1.268360 \\
\hline $\mathrm{H}$ & -7.739232 & -4.020707 & -1.560440 \\
\hline $\mathrm{H}$ & -6.343112 & -4.153528 & -2.642260 \\
\hline $\mathrm{H}$ & -5.652741 & -1.068558 & -0.477150 \\
\hline $\mathrm{H}$ & -5.834861 & -1.590688 & -2.157360 \\
\hline $\mathrm{H}$ & -7.245191 & -1.564367 & -1.087110 \\
\hline $\mathrm{C}$ & -3.684729 & 3.338501 & 2.135070 \\
\hline $\mathrm{C}$ & -3.235409 & 2.481171 & 2.873720 \\
\hline $\mathrm{C}$ & -2.696270 & 1.498591 & 3.752200 \\
\hline $\mathrm{C}$ & -3.305240 & 1.260011 & 5.002160 \\
\hline $\mathrm{C}$ & -1.541410 & 0.775160 & 3.399680 \\
\hline
\end{tabular}




$\begin{array}{lrrr}\mathrm{C} & -2.767650 & 0.318581 & 5.874200 \\ \mathrm{H} & -4.195630 & 1.819052 & 5.274560 \\ \mathrm{C} & -1.007860 & -0.160930 & 4.282310 \\ \mathrm{H} & -1.055820 & 0.946290 & 2.446000 \\ \mathrm{C} & -1.617570 & -0.392540 & 5.517900 \\ \mathrm{H} & -3.244180 & 0.140471 & 6.834670 \\ \mathrm{H} & -0.114221 & -0.700690 & 3.986370 \\ \mathrm{H} & -1.198491 & -1.124510 & 6.203460\end{array}$

\section{REFERENCES}

1. Gottlieb, H. E.; Kotlyar, V.; Nudelman, A. J. Org. Chem. 1997, 62, 7512-7515.

2. 1 a, d, e, l, n: a) Park, J. H.; Bhilare, S. V.; Youn, S. W. Org. Lett. 2011, 13, 22282231. 1b, m: b) Dyker, G.; Stirner, W.; Henkel, G. Eur. J. Org. Chem. 2000, 14331441. 1c: c) Hashmi, A. S. K.; Braun, I.; Nösel, P.; Schädlich, J.; Wieteck, M.; Rudolph, M.; Rominger, F. Angew. Chem. Int. Ed. 2012, 51, 4456-4460. 1f: d) Schmittel, M.; Vavilala, C. J. Org. Chem. 2005, 70, 4865-4868. 1h: e) Alfonsi, M.; Dell'Acqua, M.; Facoetti, D.; Arcadi, A.; Abbiati, G.; Rossi, E. Eur. J. Org. Chem. 2009, 2852-2862. 1i: f) Patil, N. T.; Mutyala, A. K.; Lakshmi, P. G. V. V.; Raju, P. V. K.; Sridhar, B. Eur. J. Org. Chem. 2010, 1999-2007. 1j: g) Saifuddin, M.; Samala, S.; Venkata Krishna, D. G.; Kundu, B. Synthesis 2013, 45, 1553-1563. 1p: h) Zhu, S.; Zhang, Z.; Huang, X.; Jiang, H.; Guo, Z. Chem. Eur. J. 2013, 19, 46954700.

3. Blanco-Urgoiti, J.; Abdi, D.; Domínguez, G.; Pérez-Castells, J. Tetrahedron 2008, 64, 67-74.

4. Asao, N.; Asano, T.; Ohishi, T.; Yamamoto, Y. J. Am. Chem. Soc. 2000, 122, 48174818.

5. CrysAlisPro, Agilent Technologies,Version 1.171.37.31 (release 14-01-2014 CrysAlis171 .NET)(compiled Jan 14 2014,18:38:05).

6. Palatinus, L.; Chapuis, G. J. Appl. Cryst. 2007, 40, 786-790.

7. Sheldrick, G. M. Acta Cryst. 2008, A64,112.

8. Macrae, C. F.; Bruno, I. J.; Chisholm, J. A.; Edgington, P. R.; McCabe, P.; Pidcock, E.; Rodriguez-Monge, L.; Taylor, R.; van de Streek, J.; Wood, P. A. J. Appl. Cryst. 2008, 41, 466-470.

9. Spek, A. L. (2010) PLATON, A Multipurpose Crystallographic Tool, Utrecht University, Utrecht, The Netherlands; A. L. Spek, J. Appl. Cryst. 2003, 36, 7-13.

10. Farrugia, L. J. J. Appl. Cryst. 1999, 32, 837-838.

11. Hooft, R. W. W.; Straver, L. H.; Spek, A. L. J. Appl. Cryst. 2008, 41, 96-103.

12. Dolomanov, O. V.; Bourhis, L. J.; Gildea, R. J.; Howard, J. A. K.; Puschmann, H. 2009 J. Appl. Cryst, 42, 339-341. 


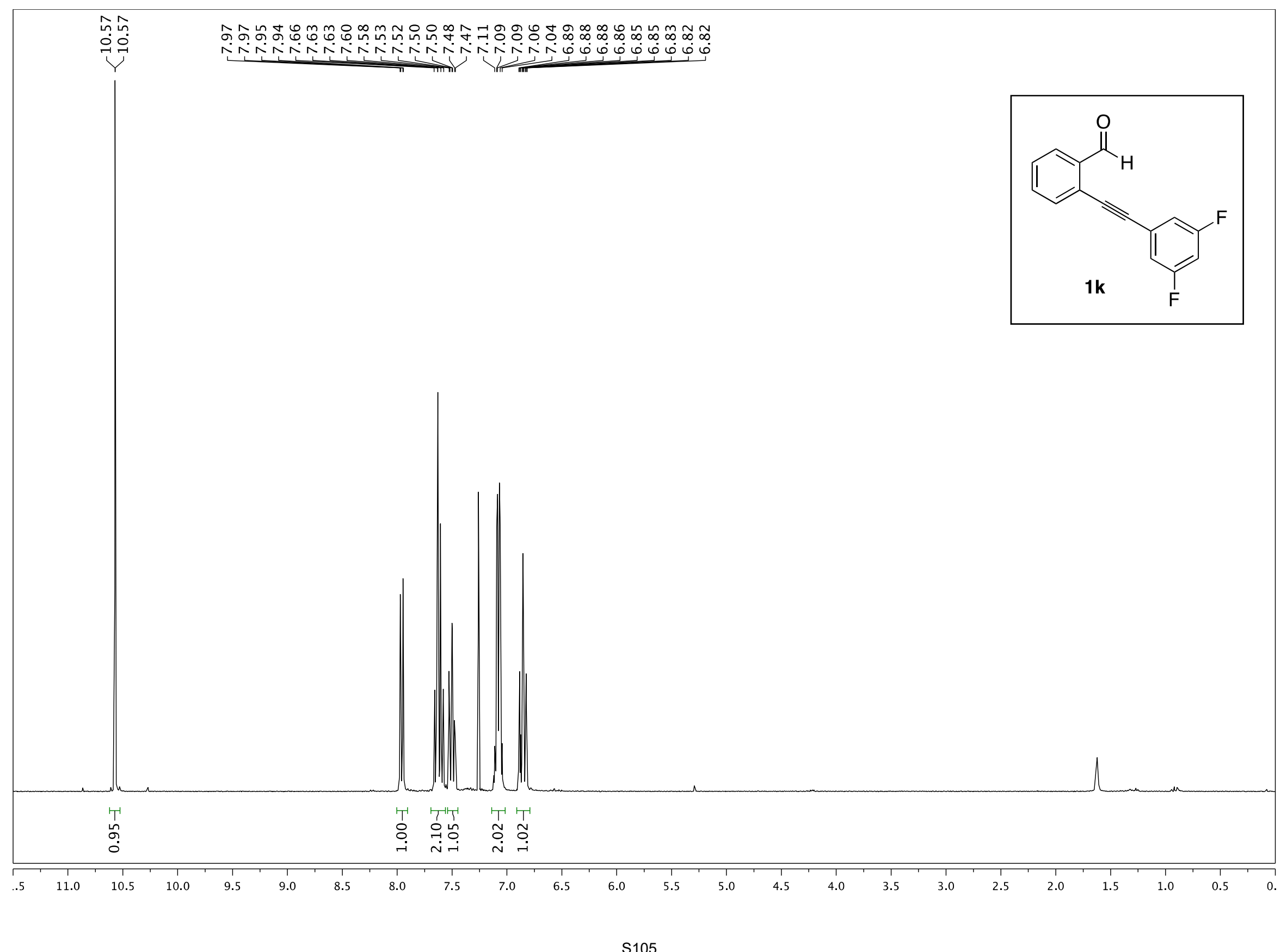




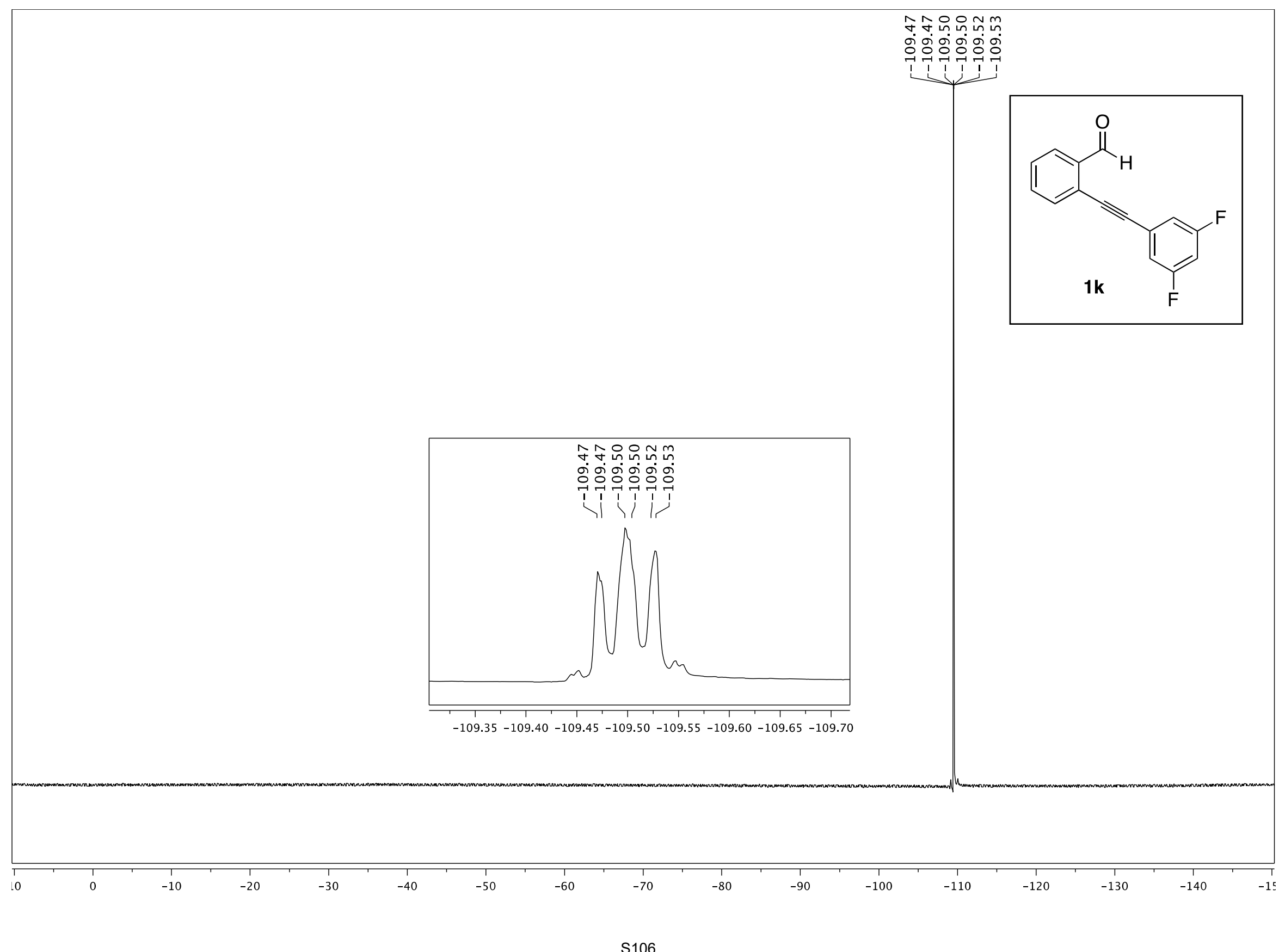




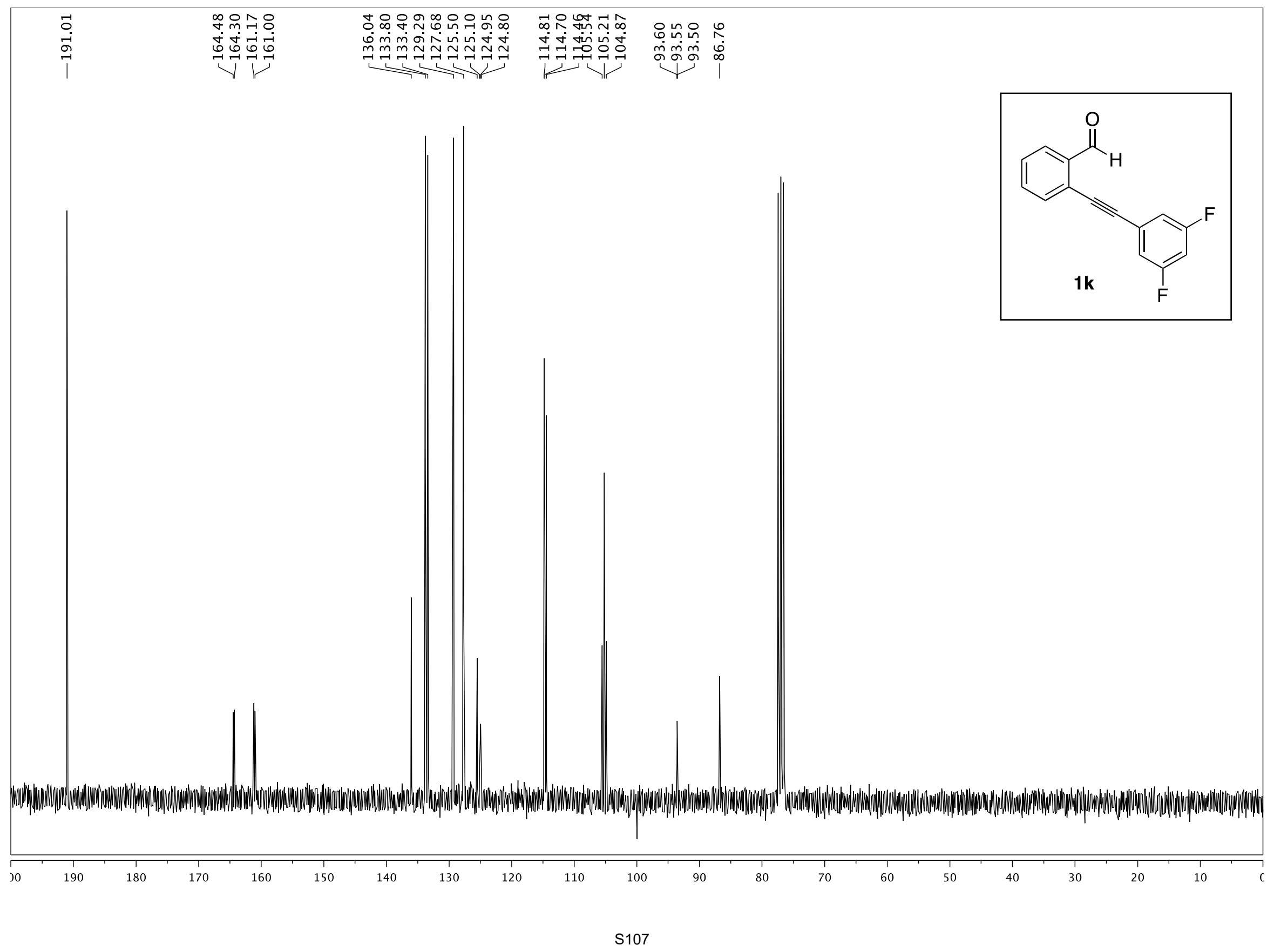




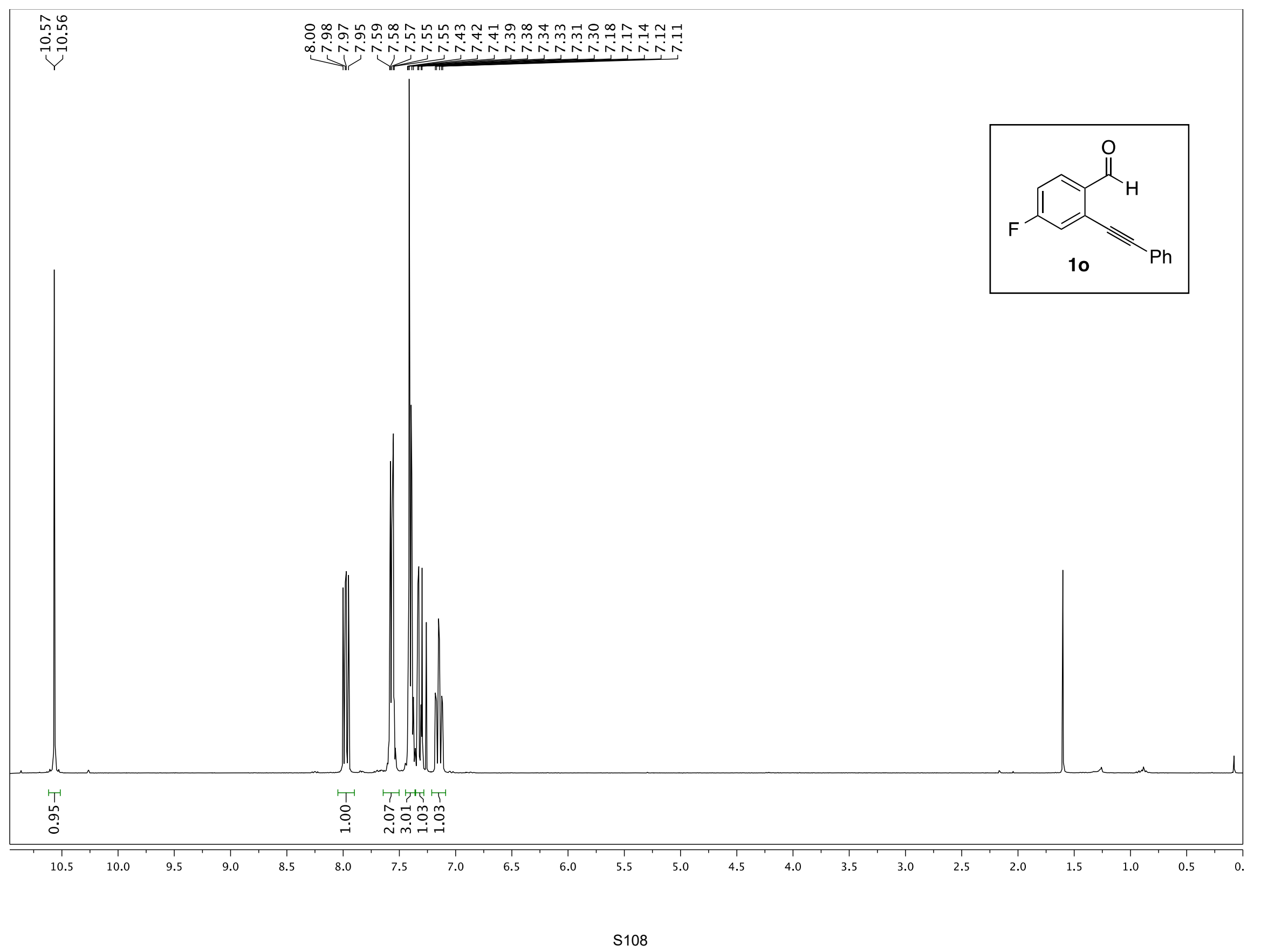




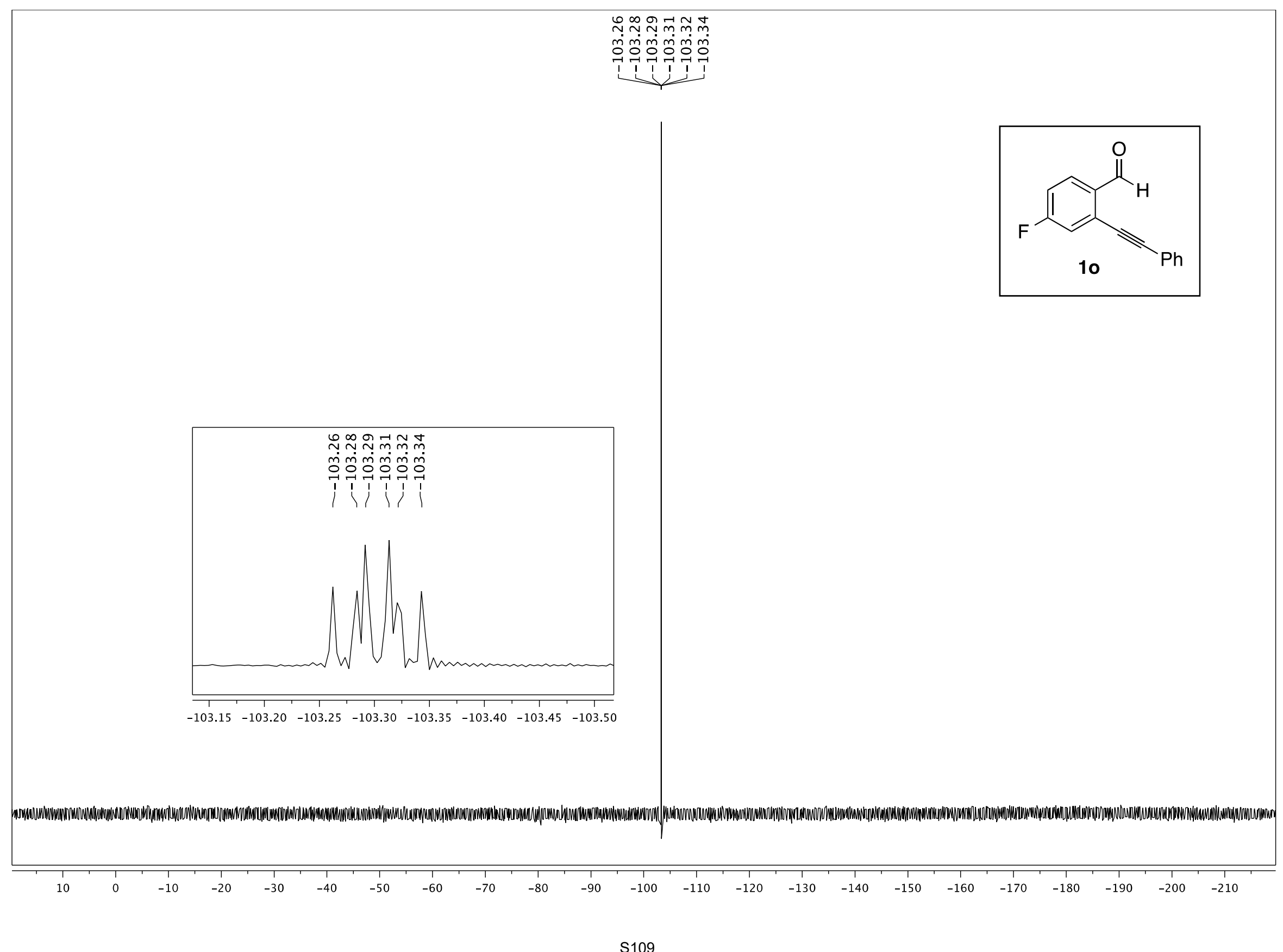




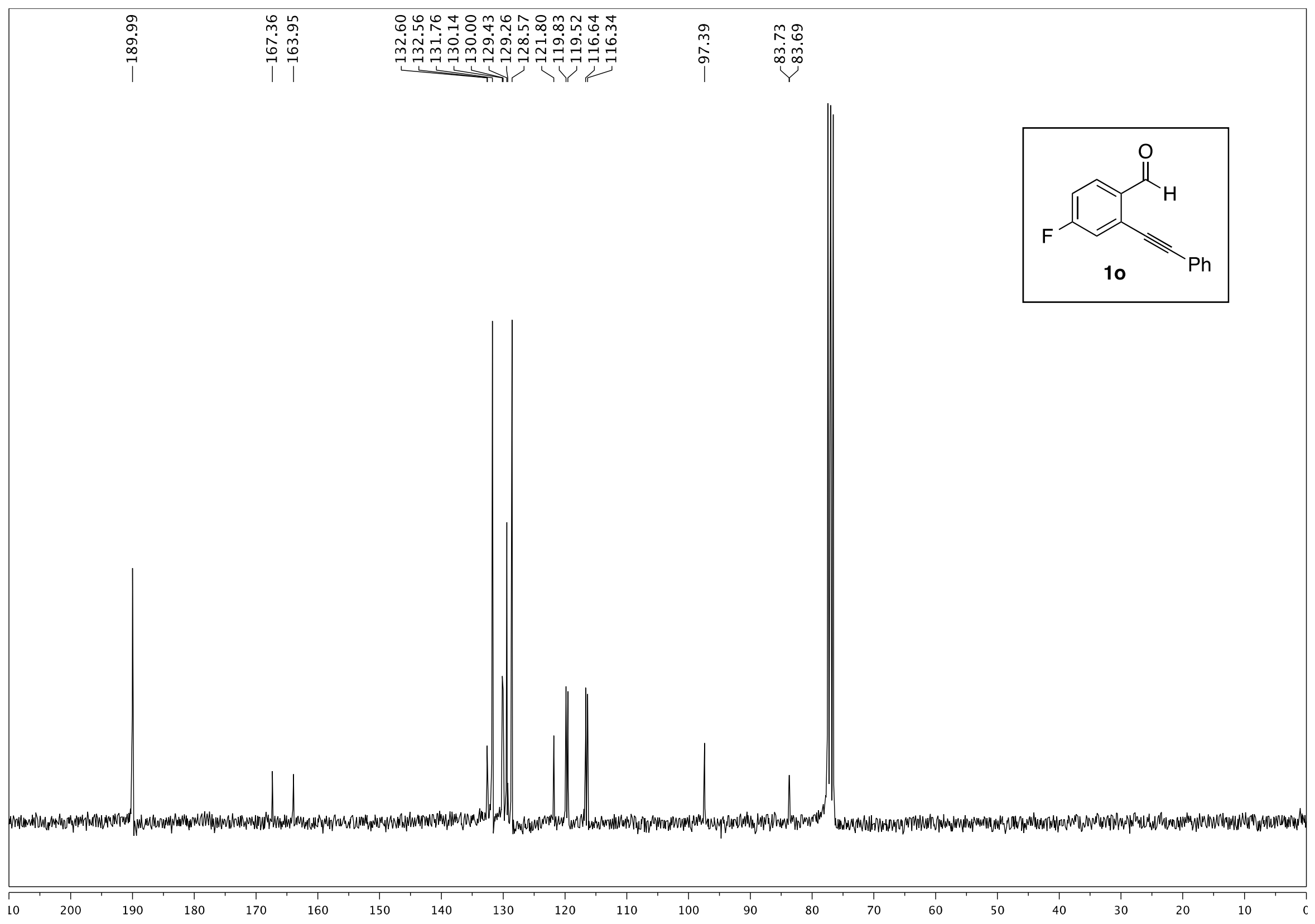




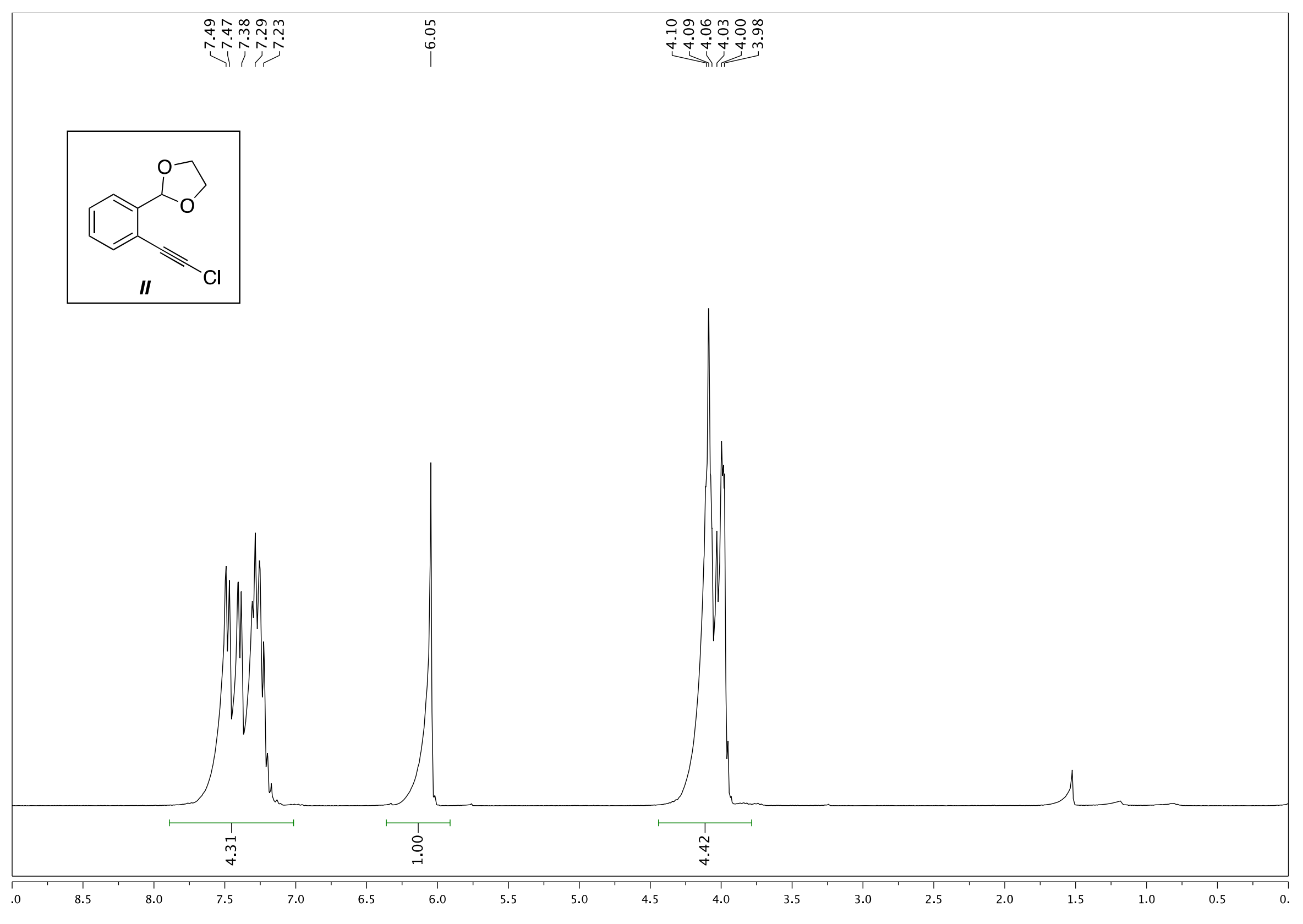




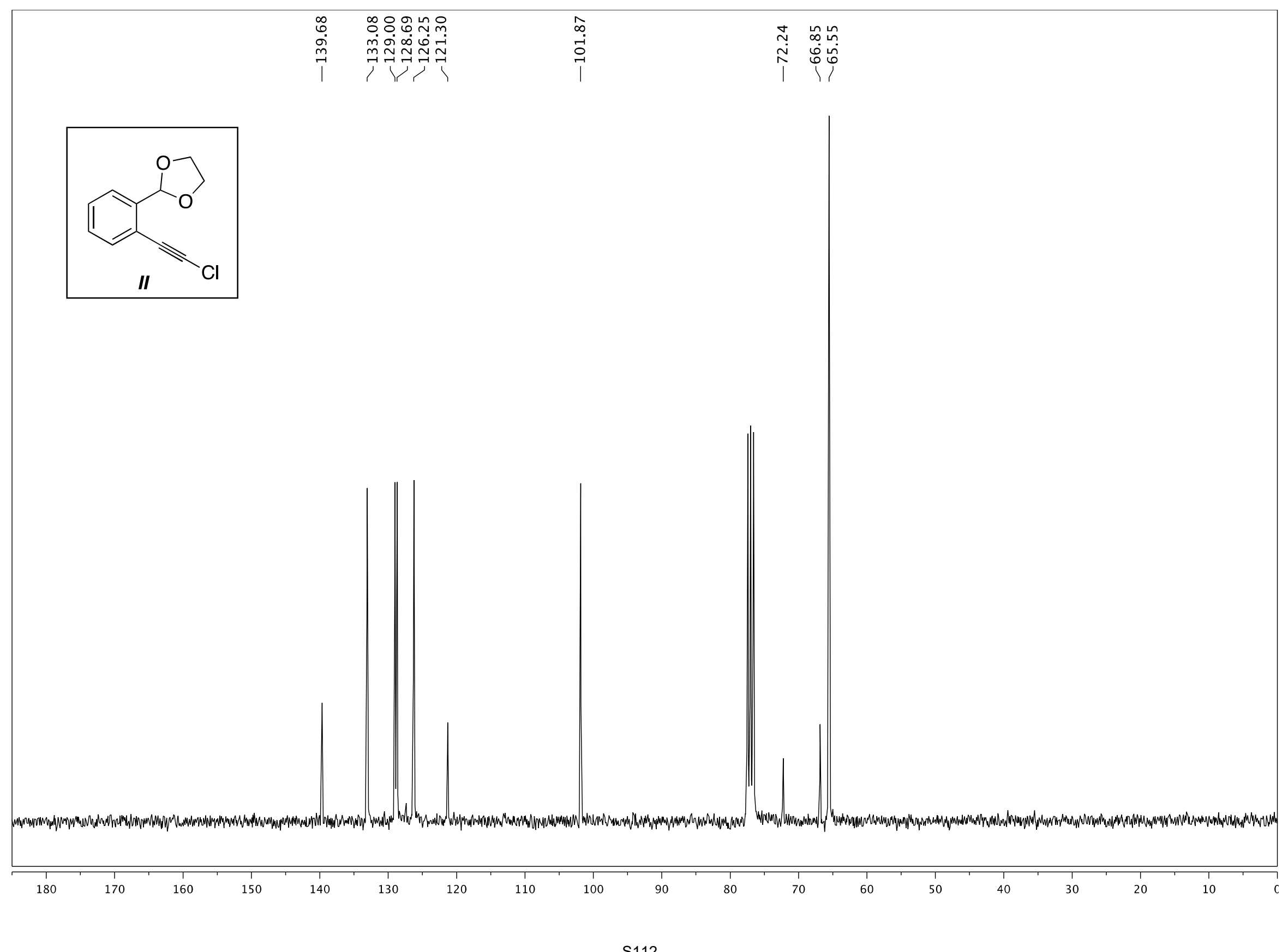




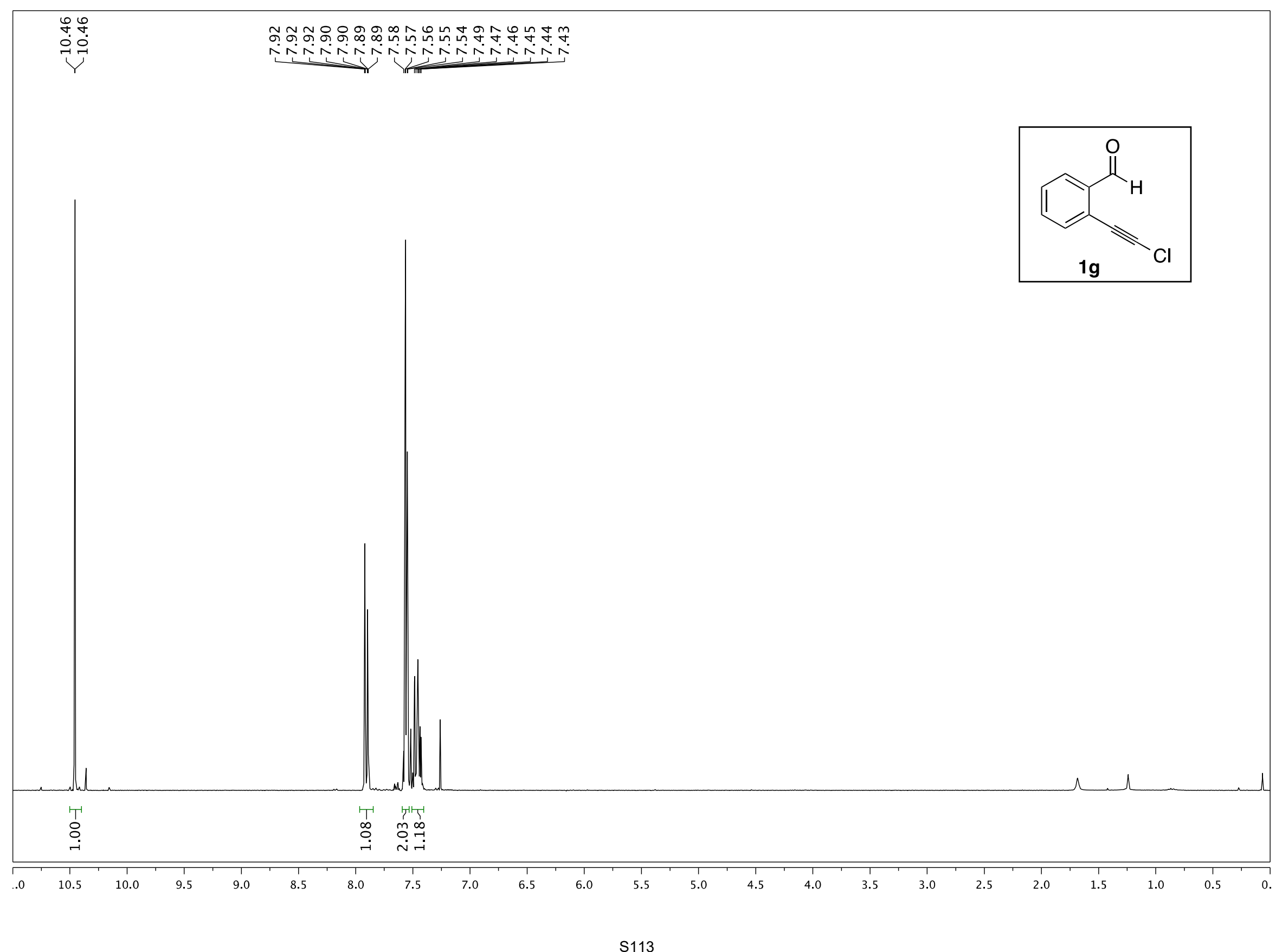




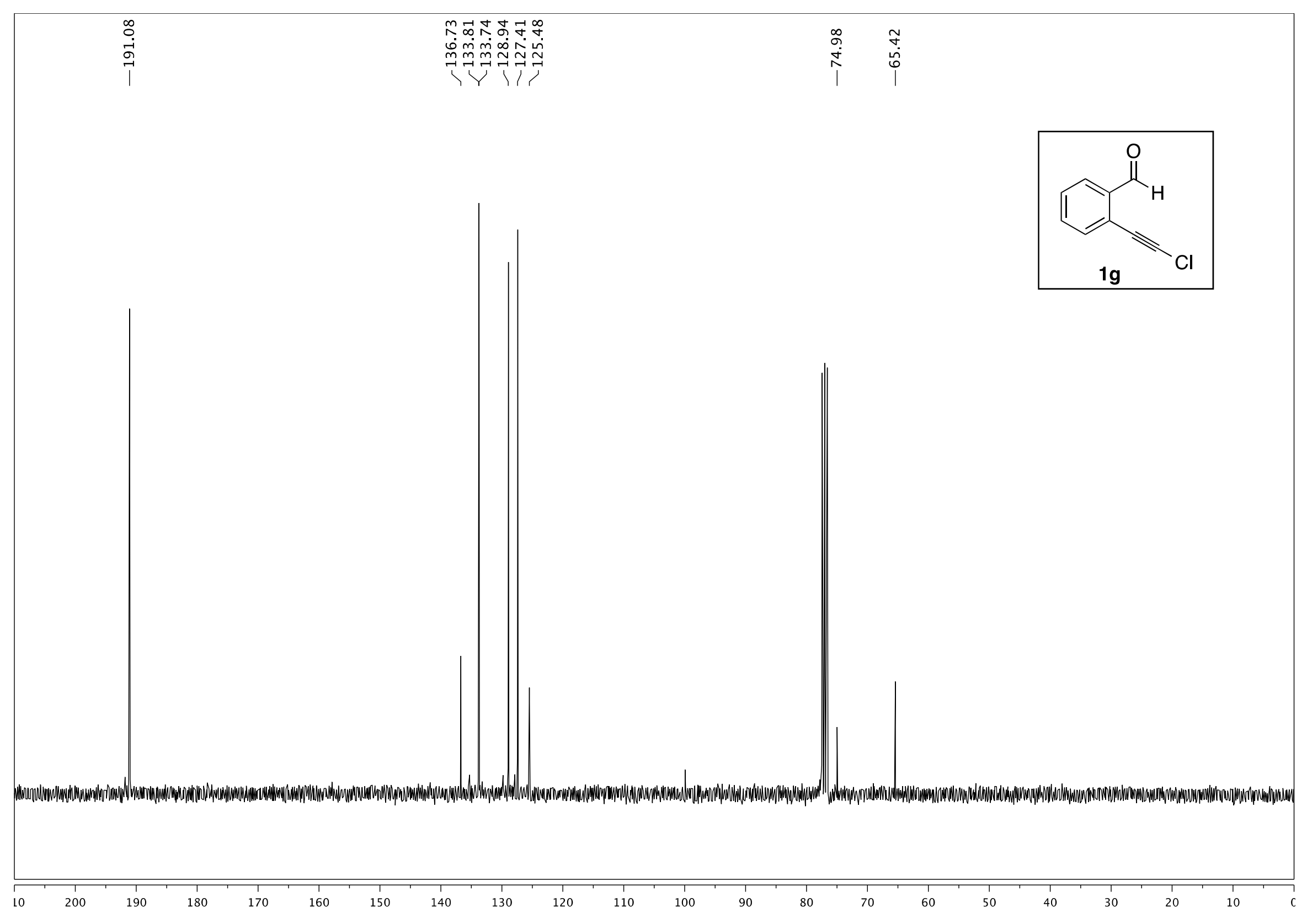




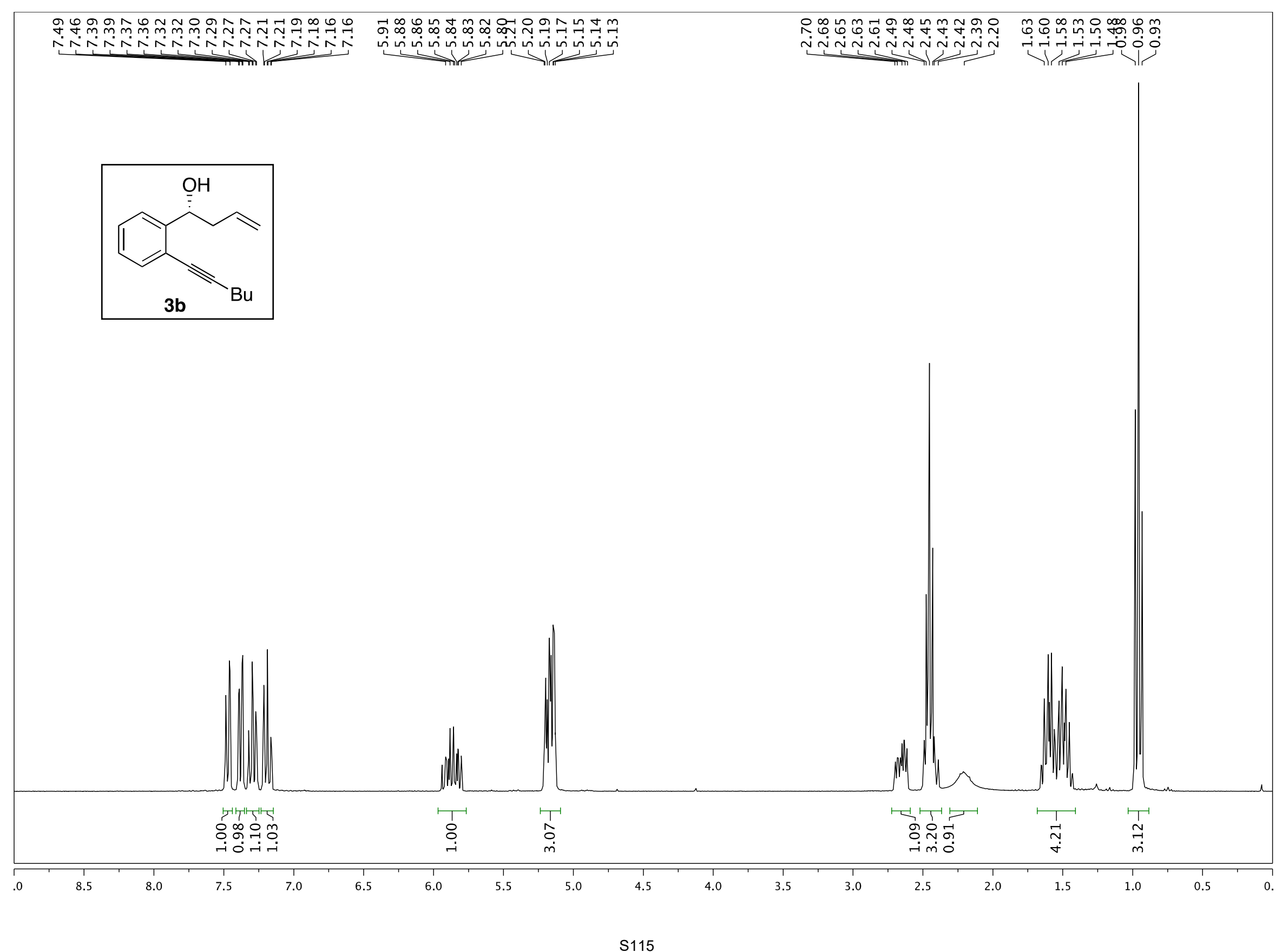




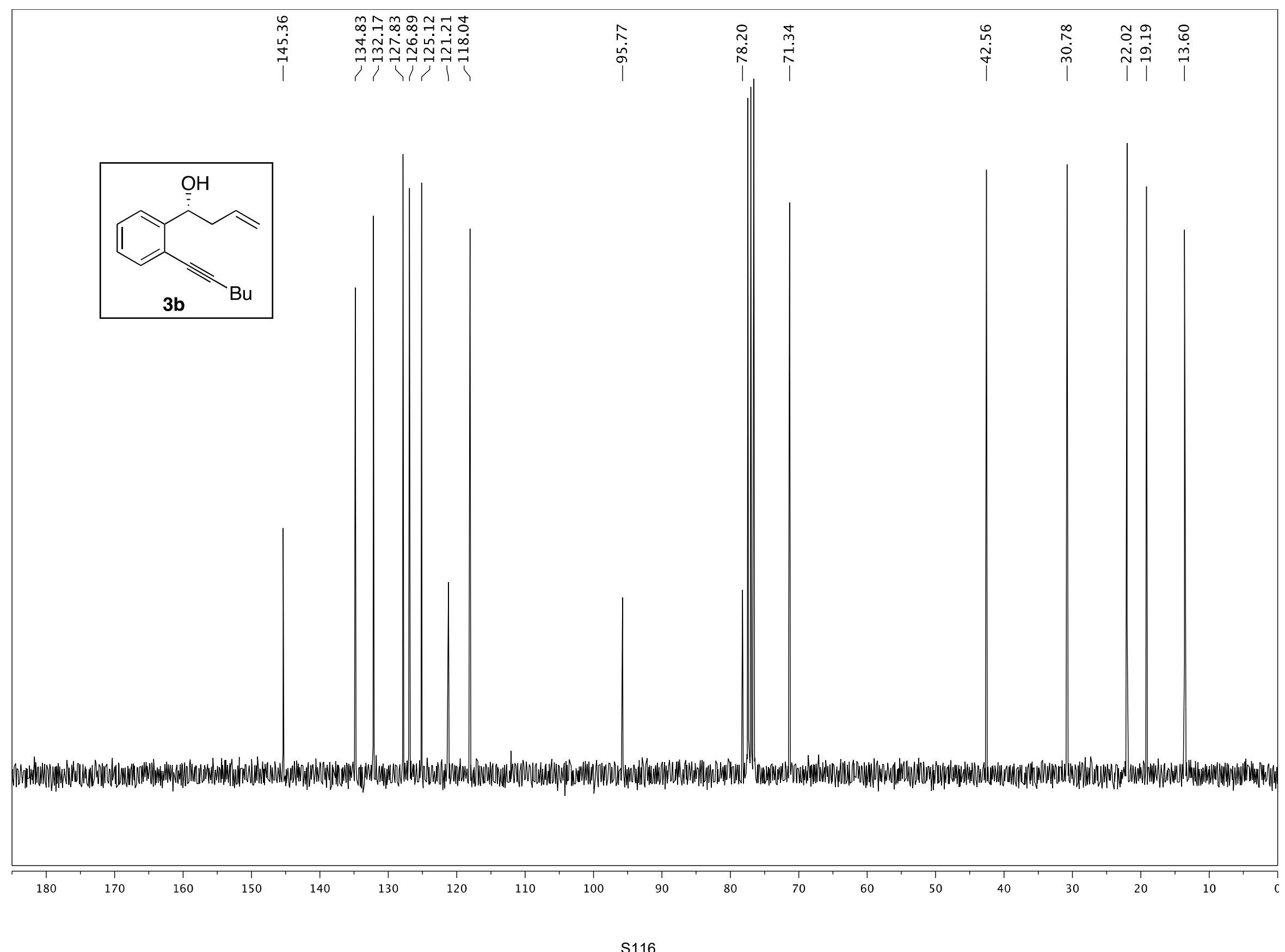




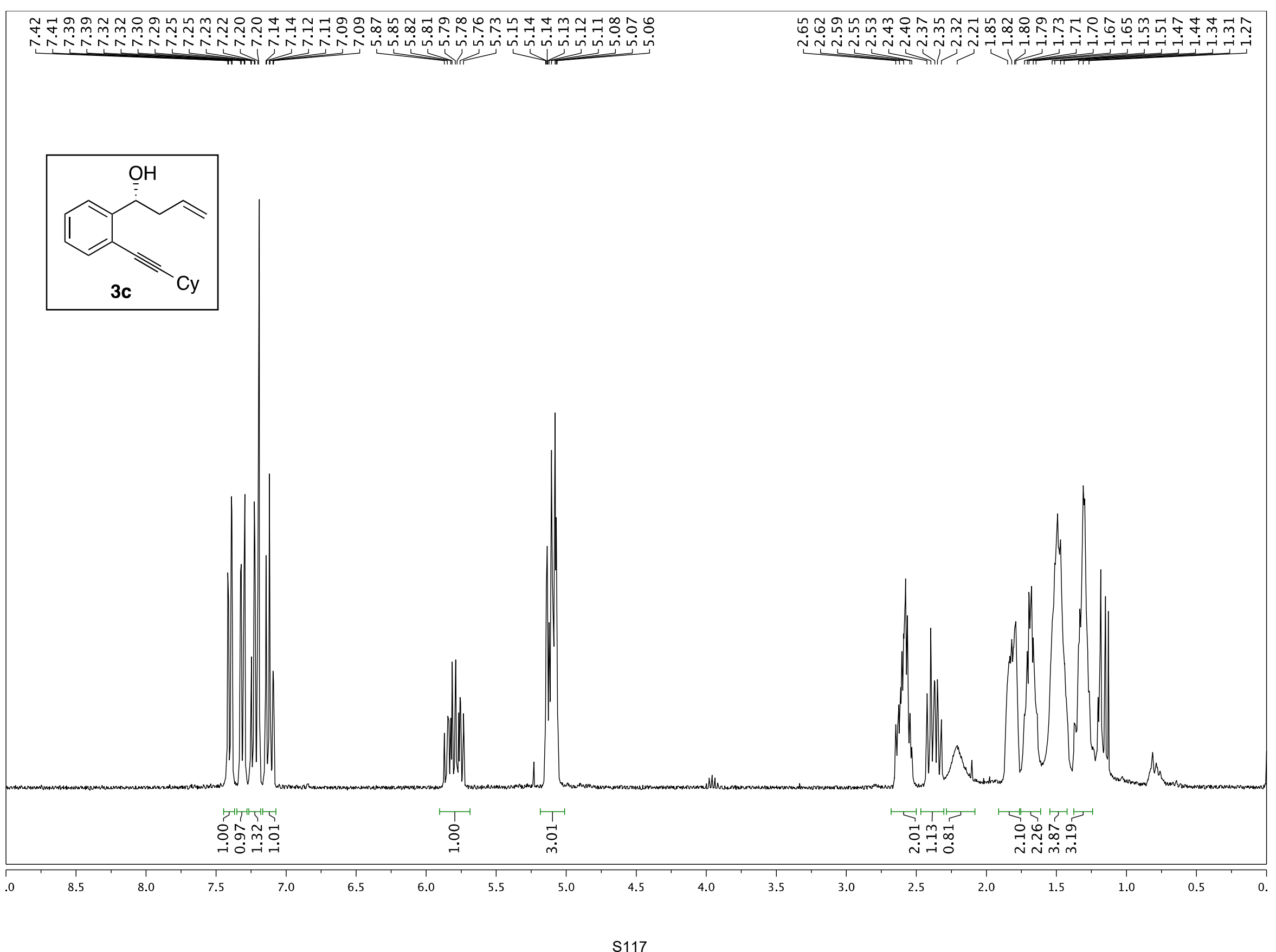




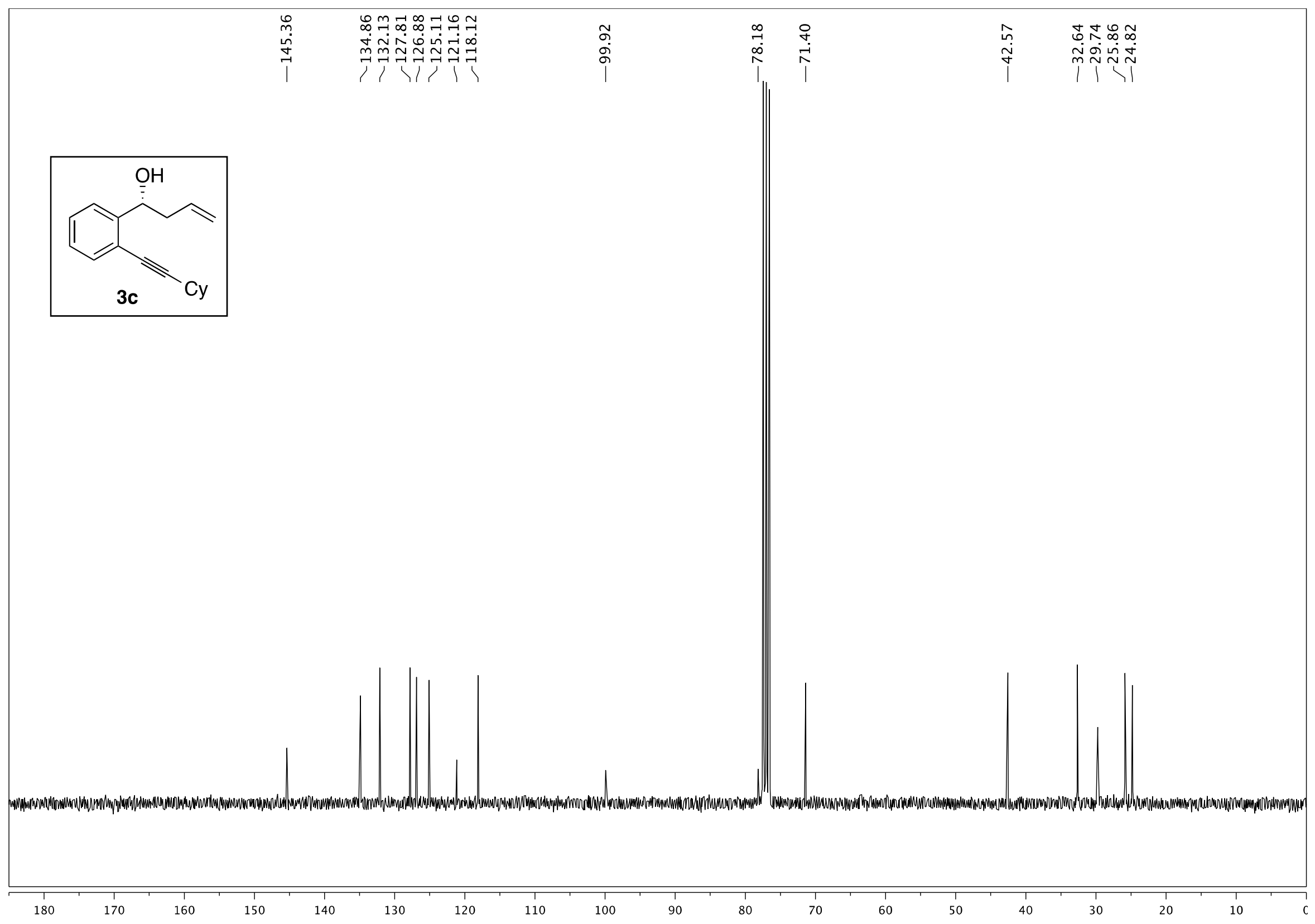




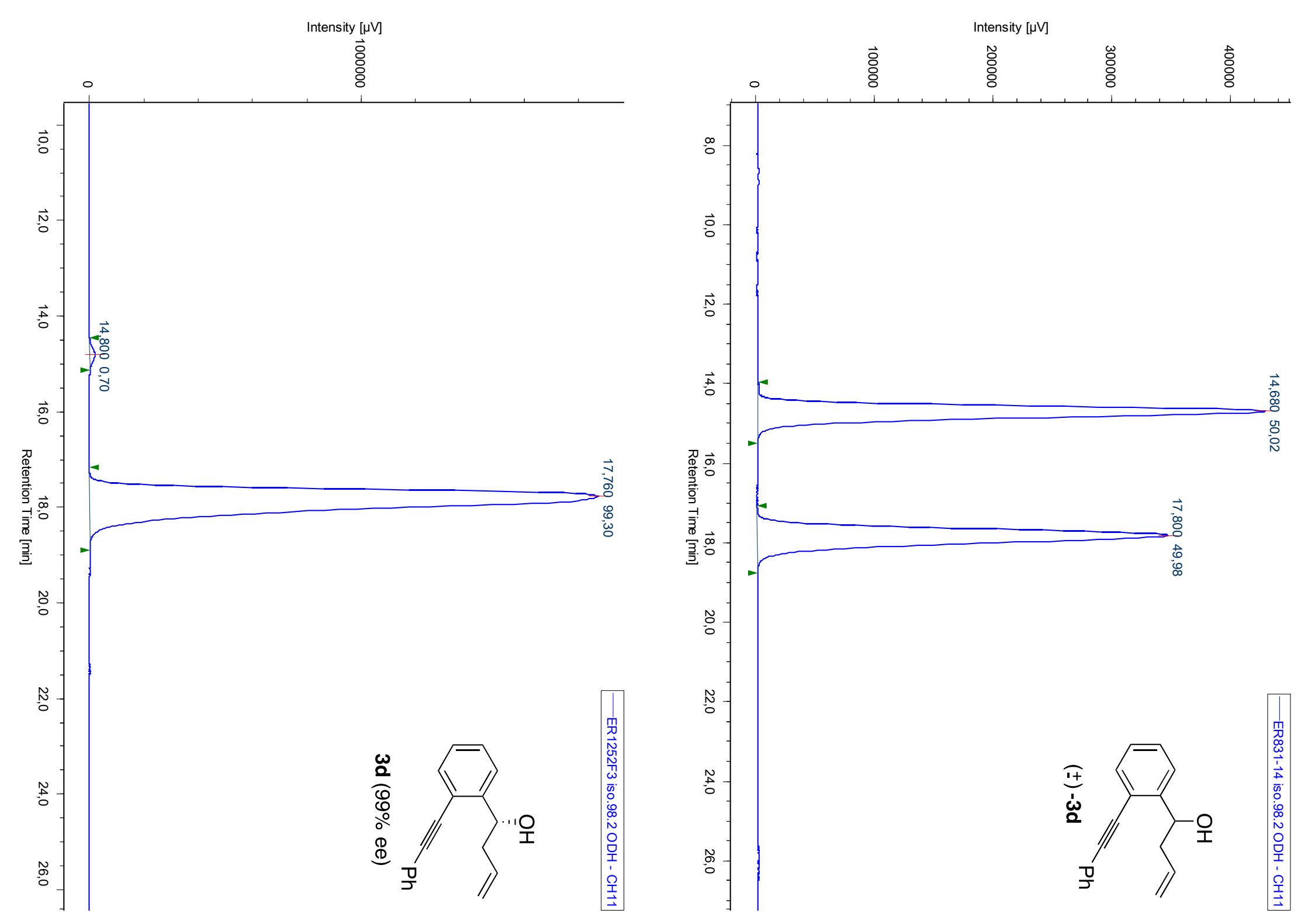




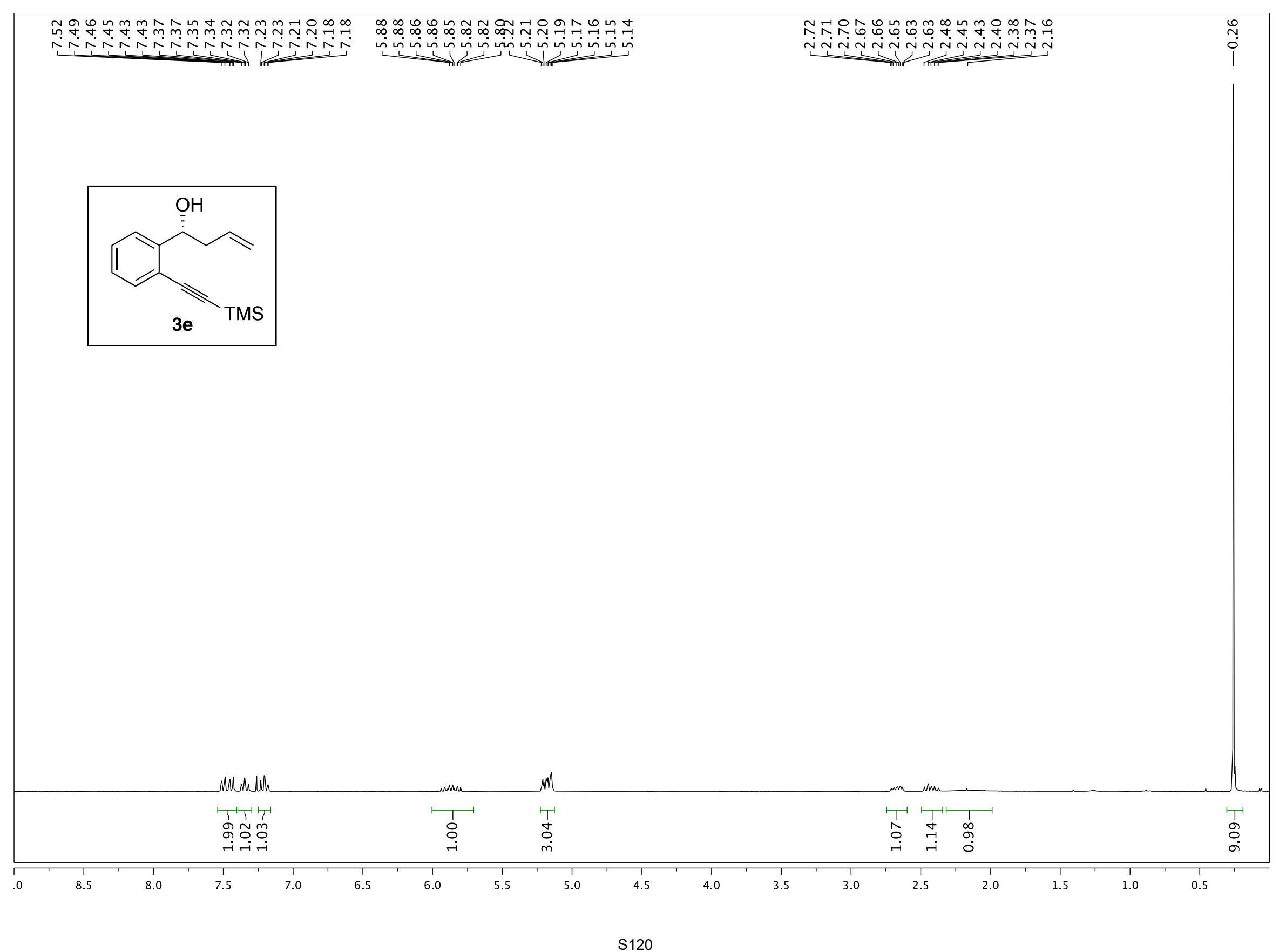




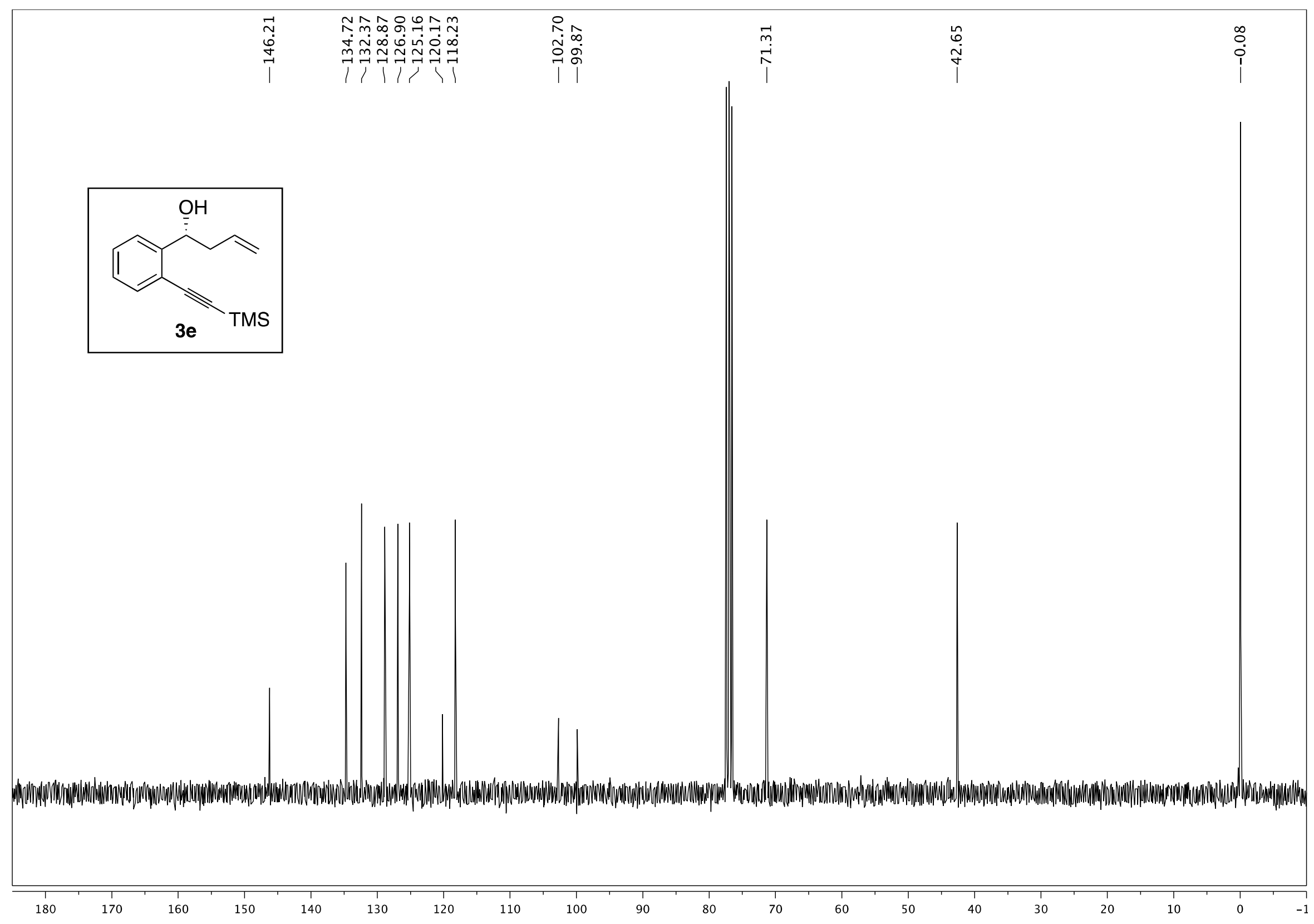




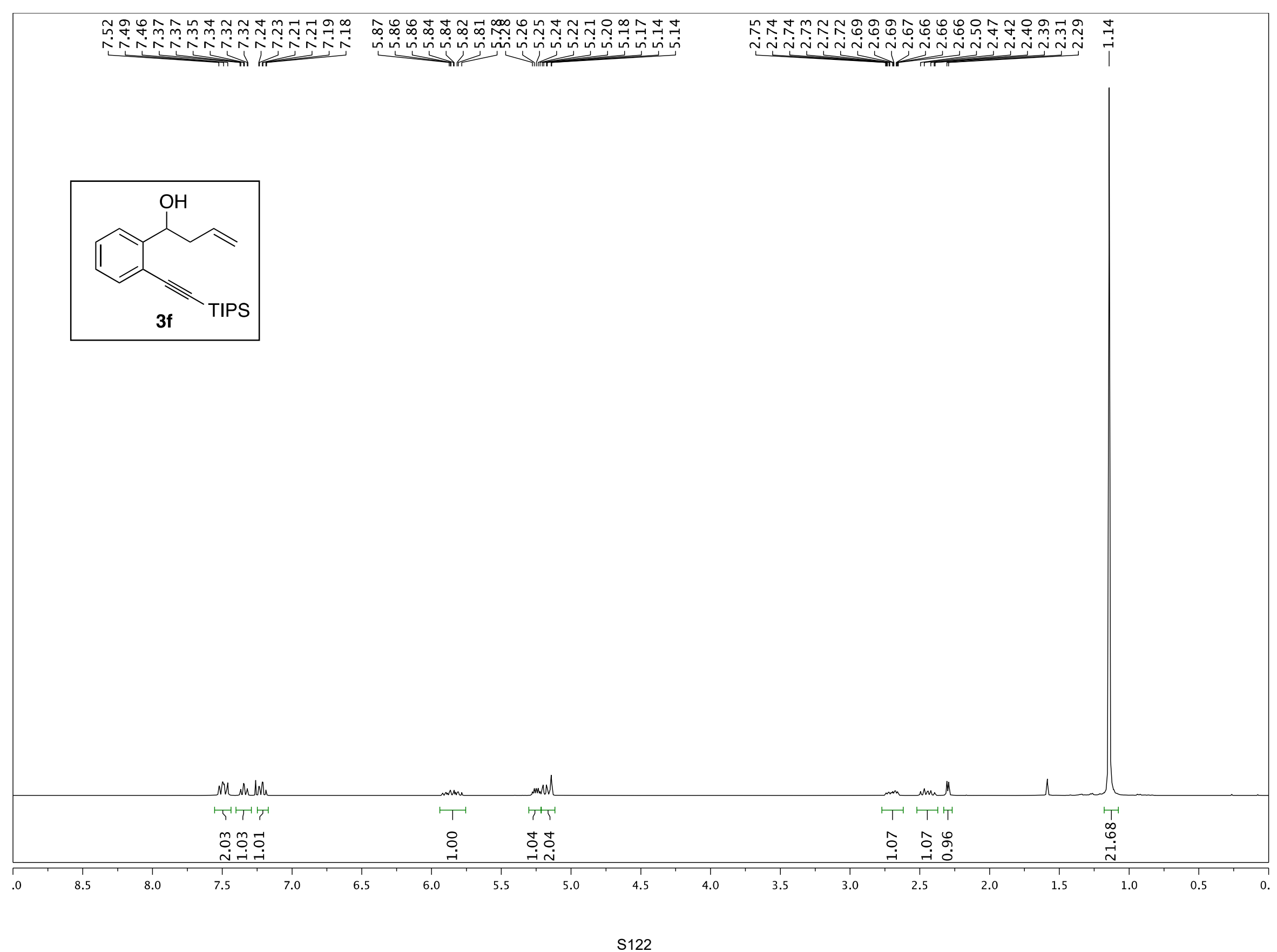




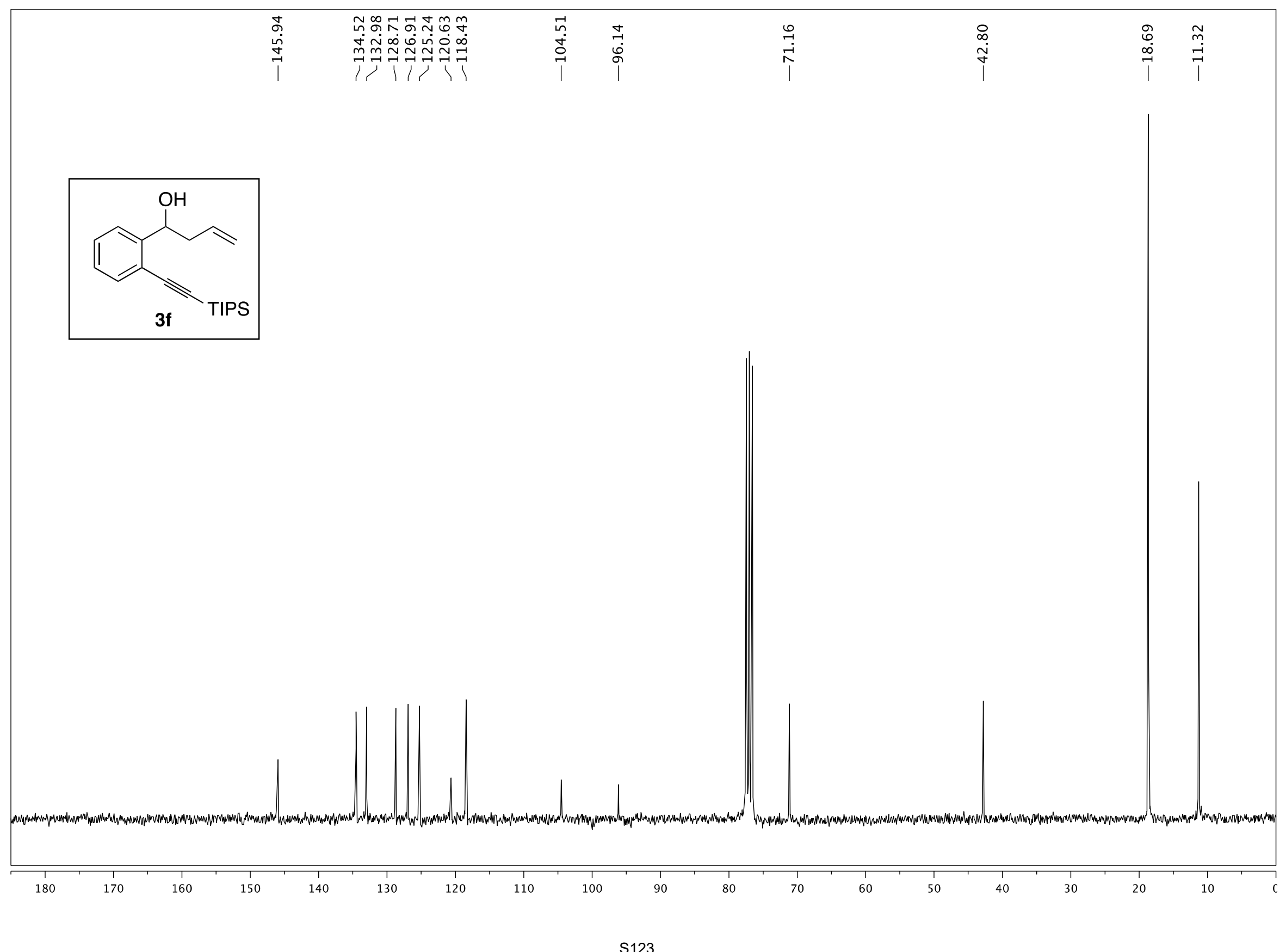




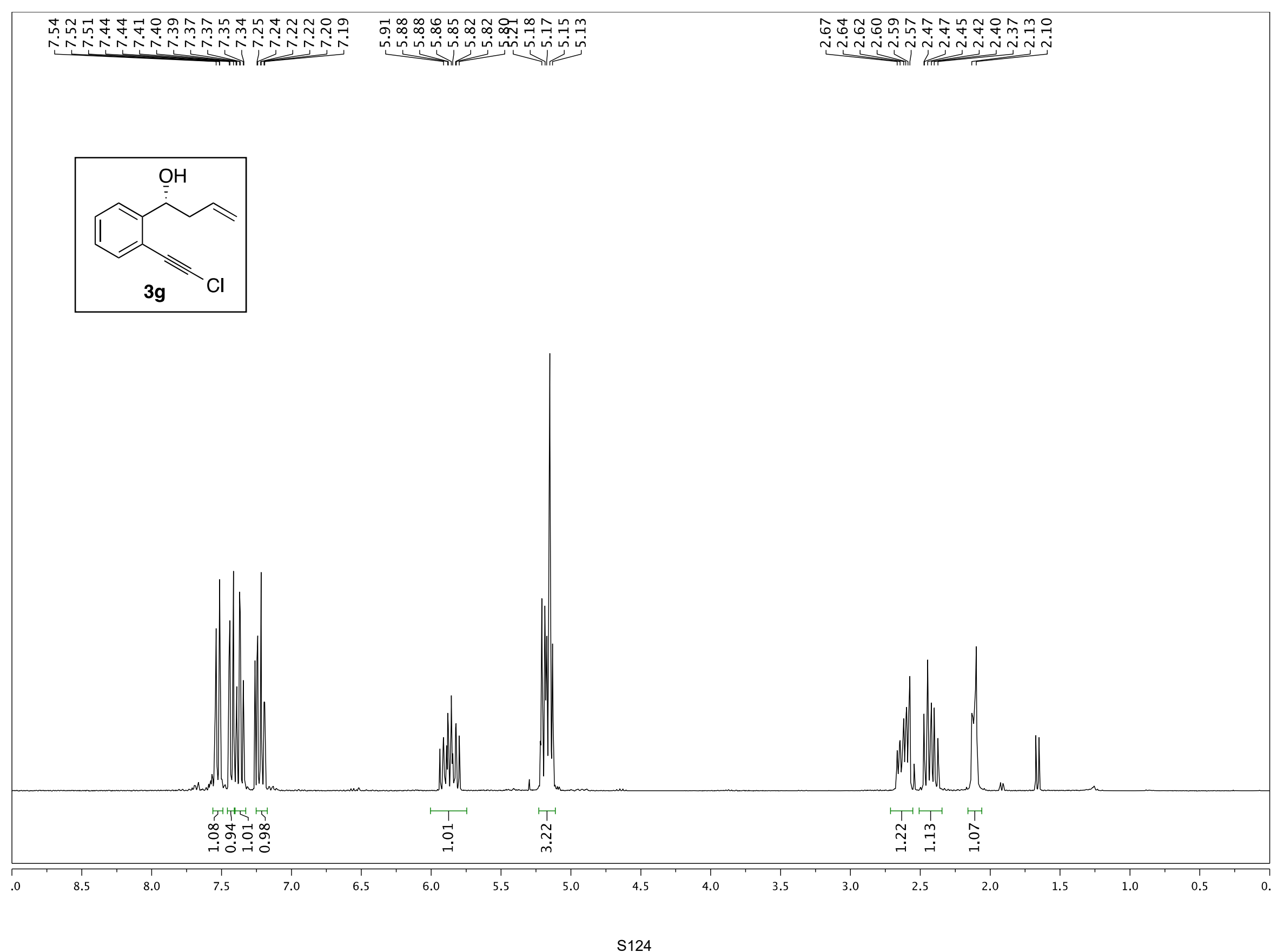




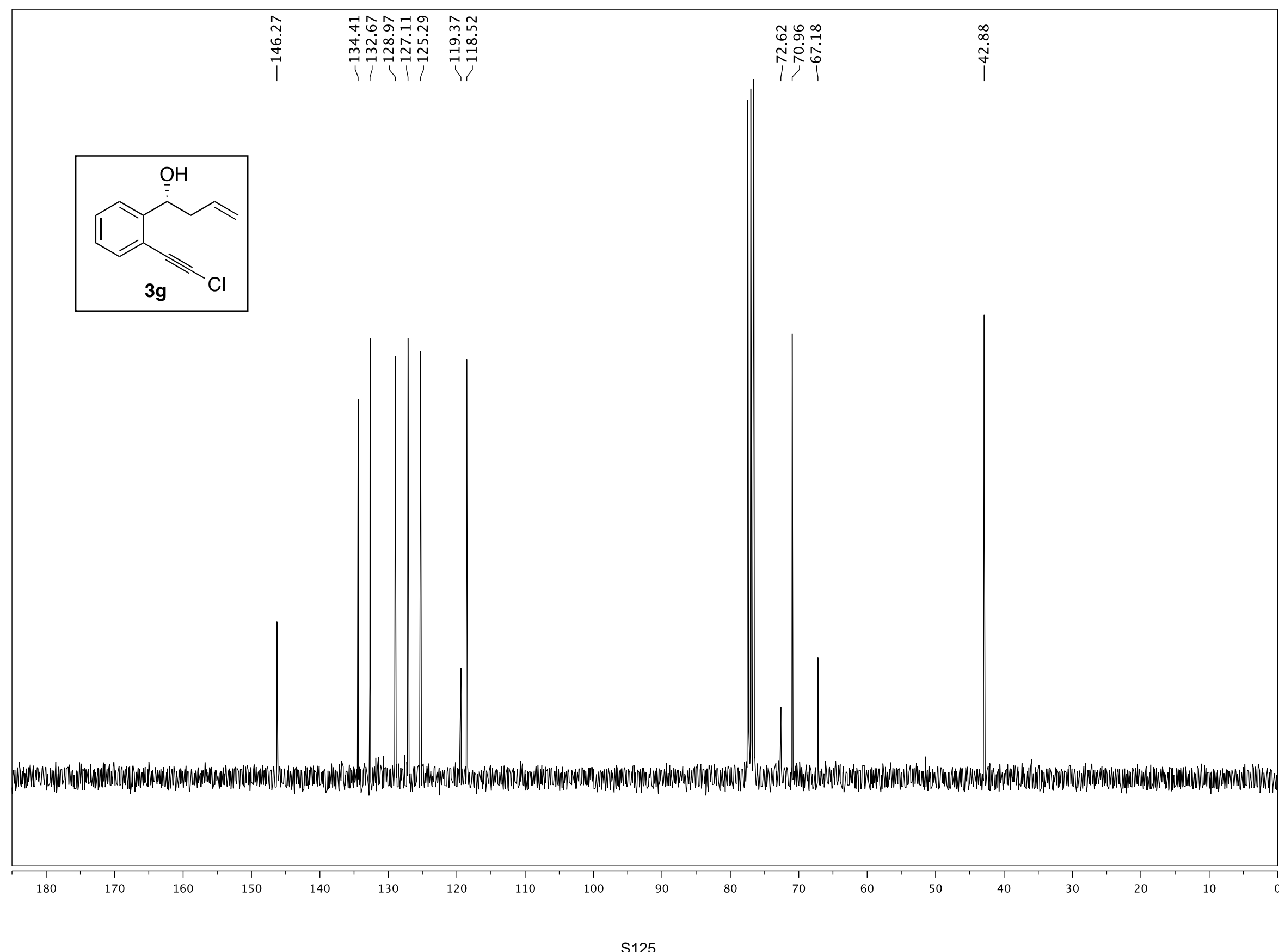




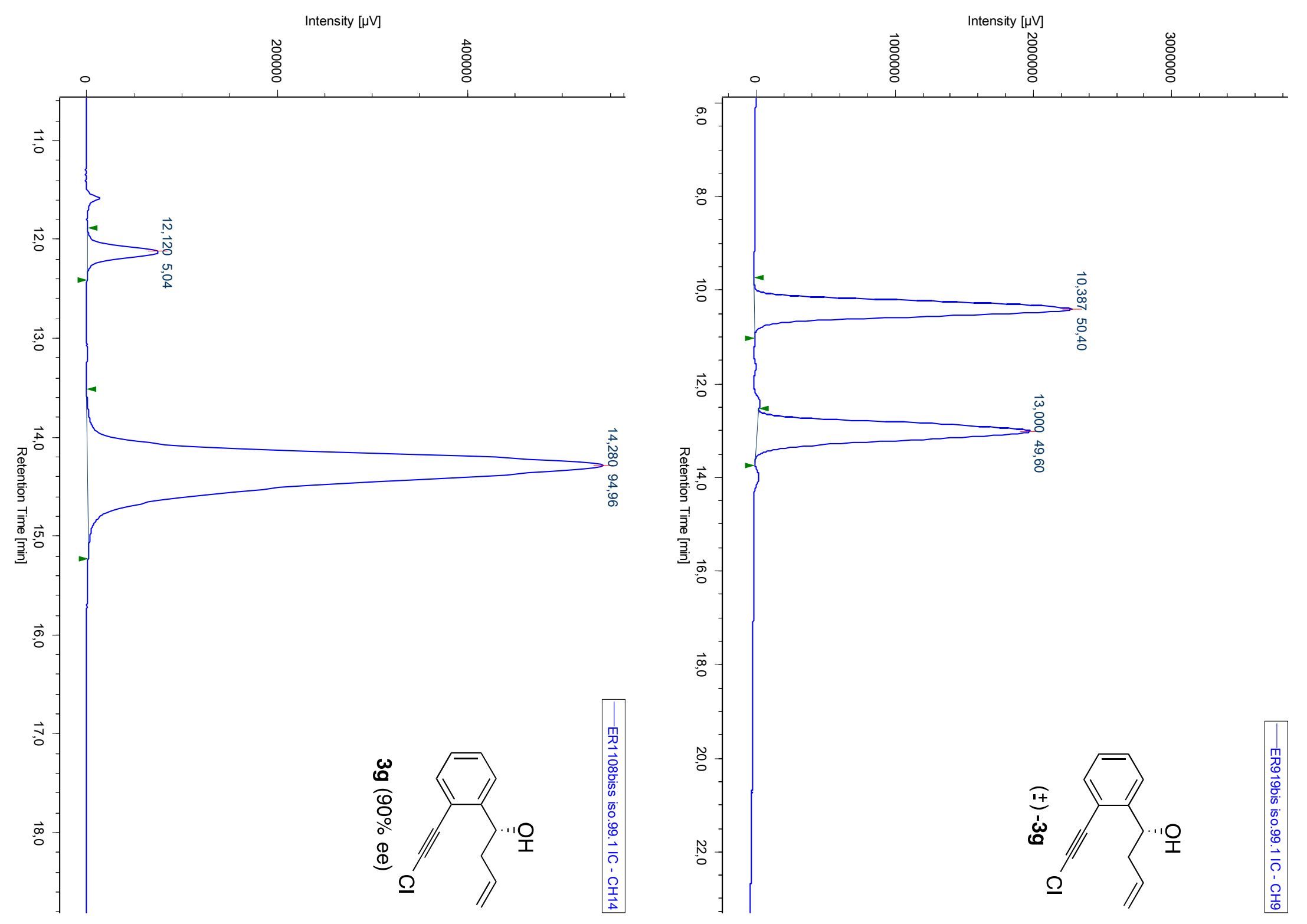




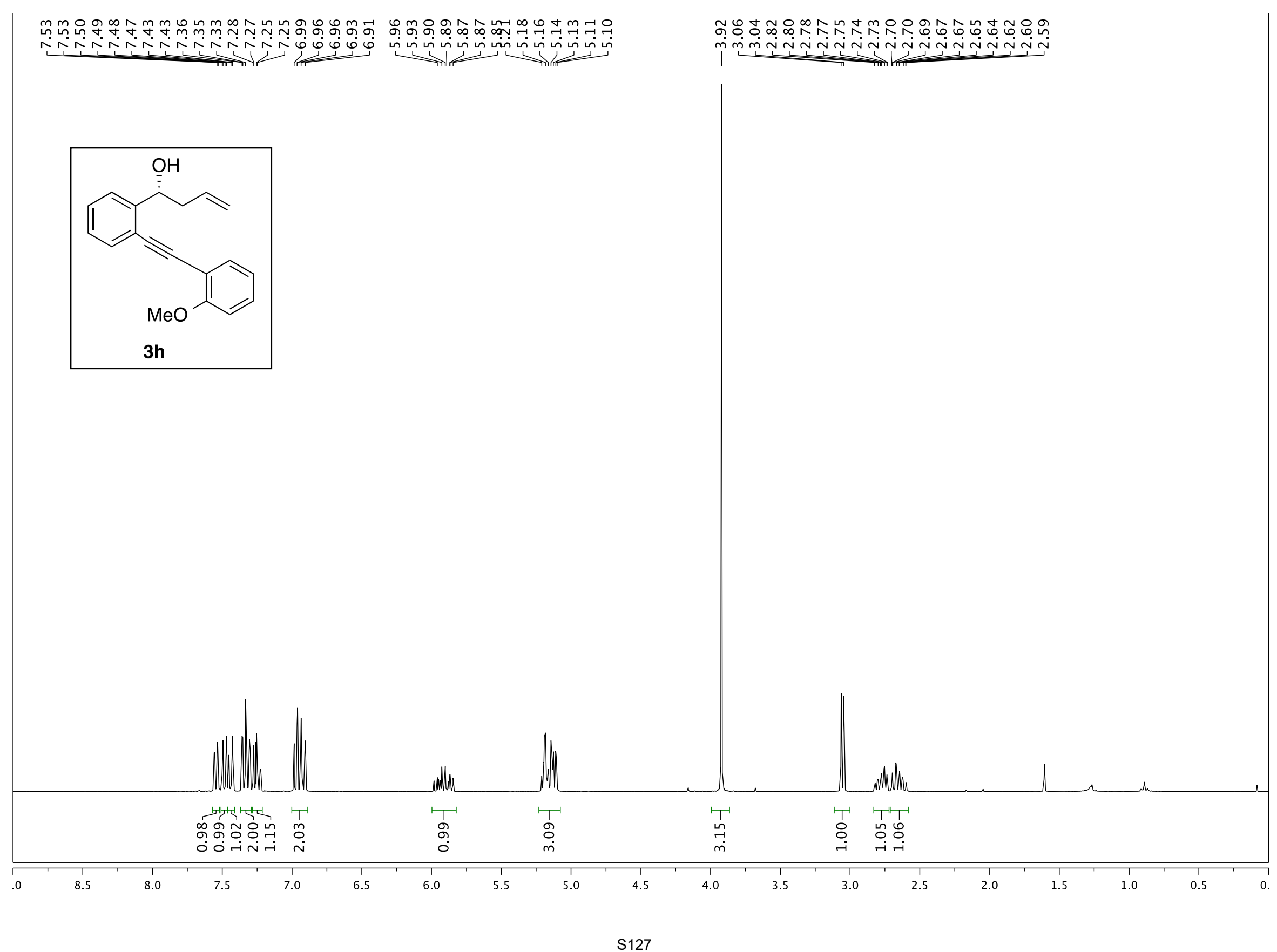




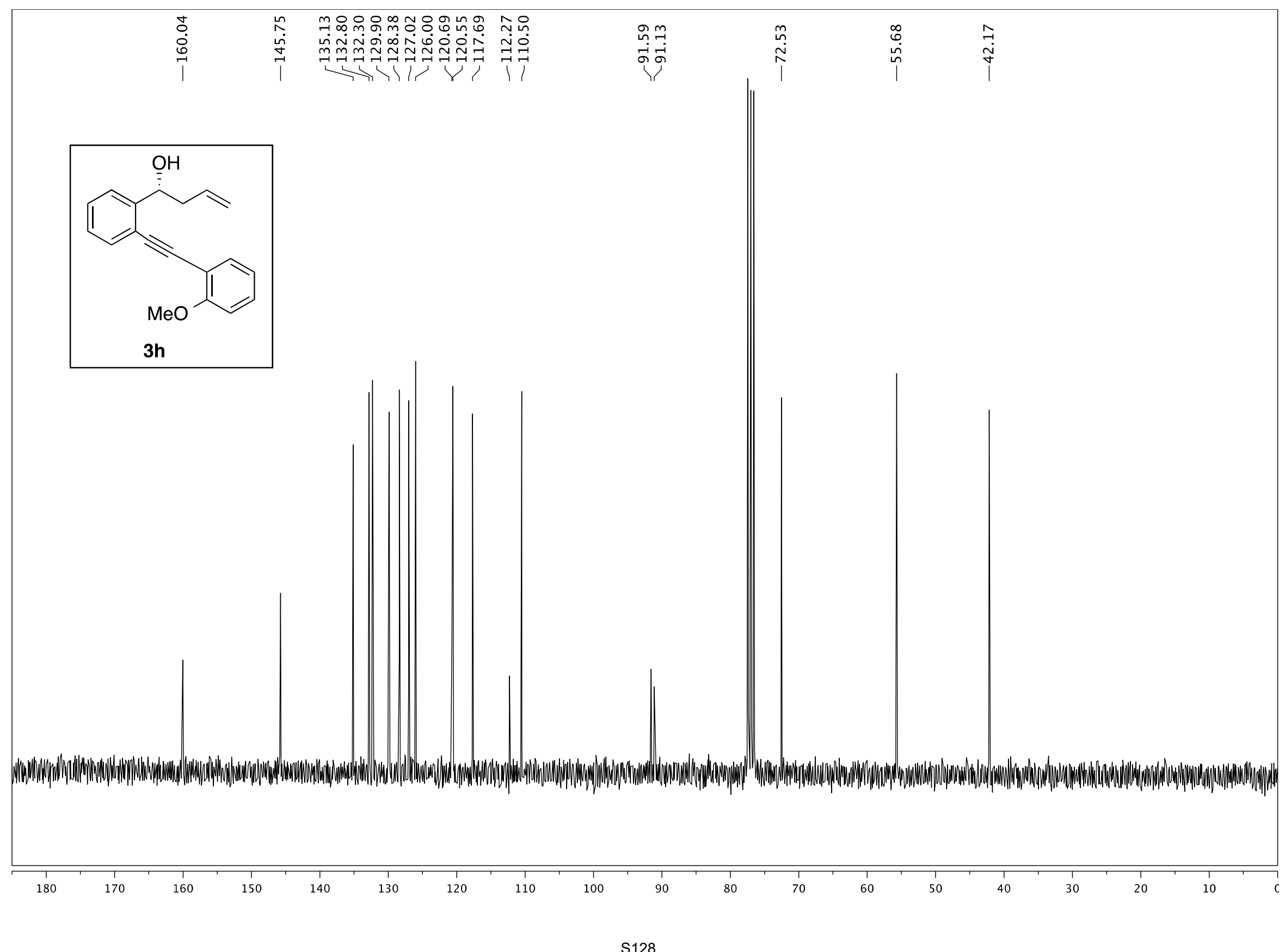




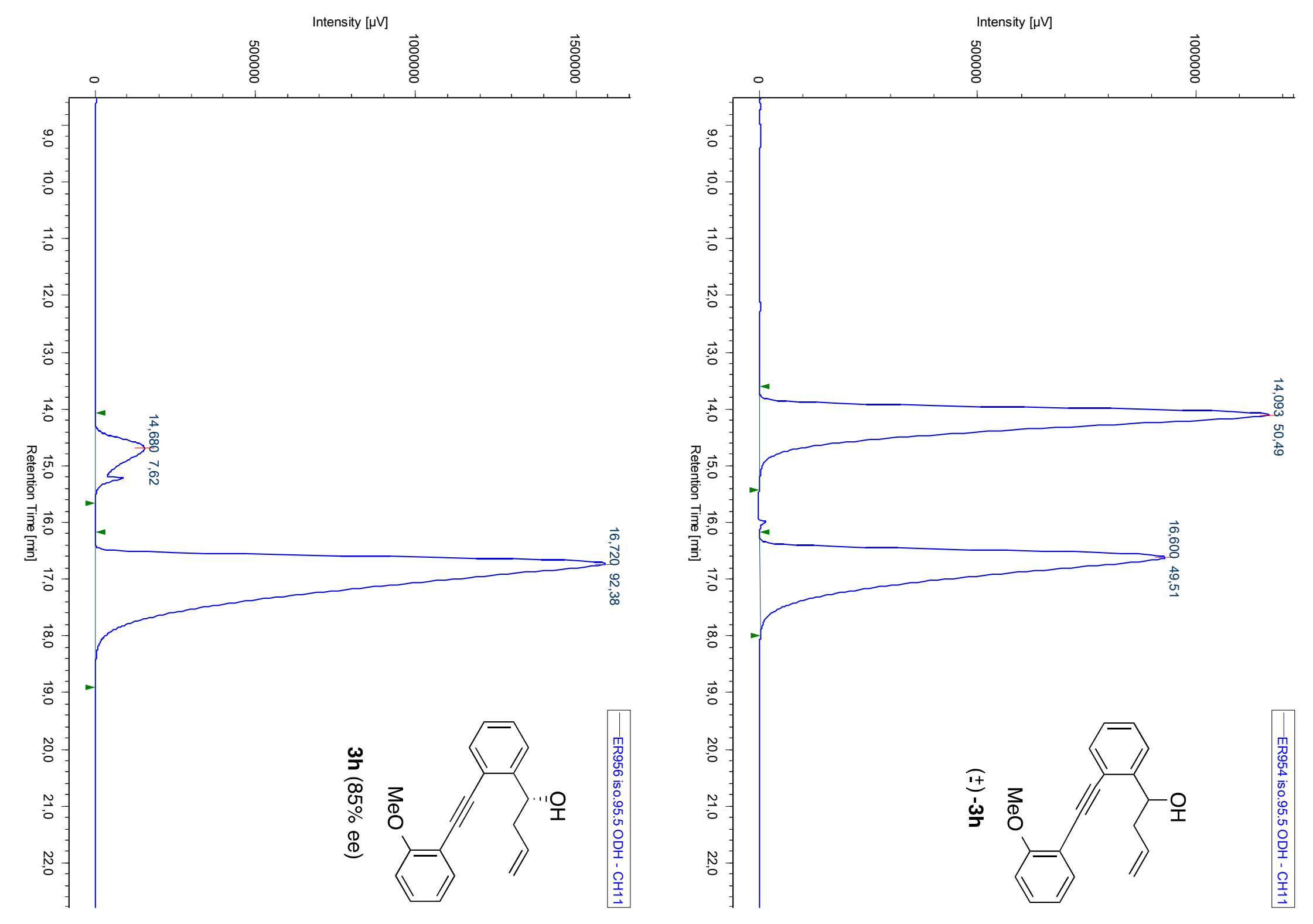




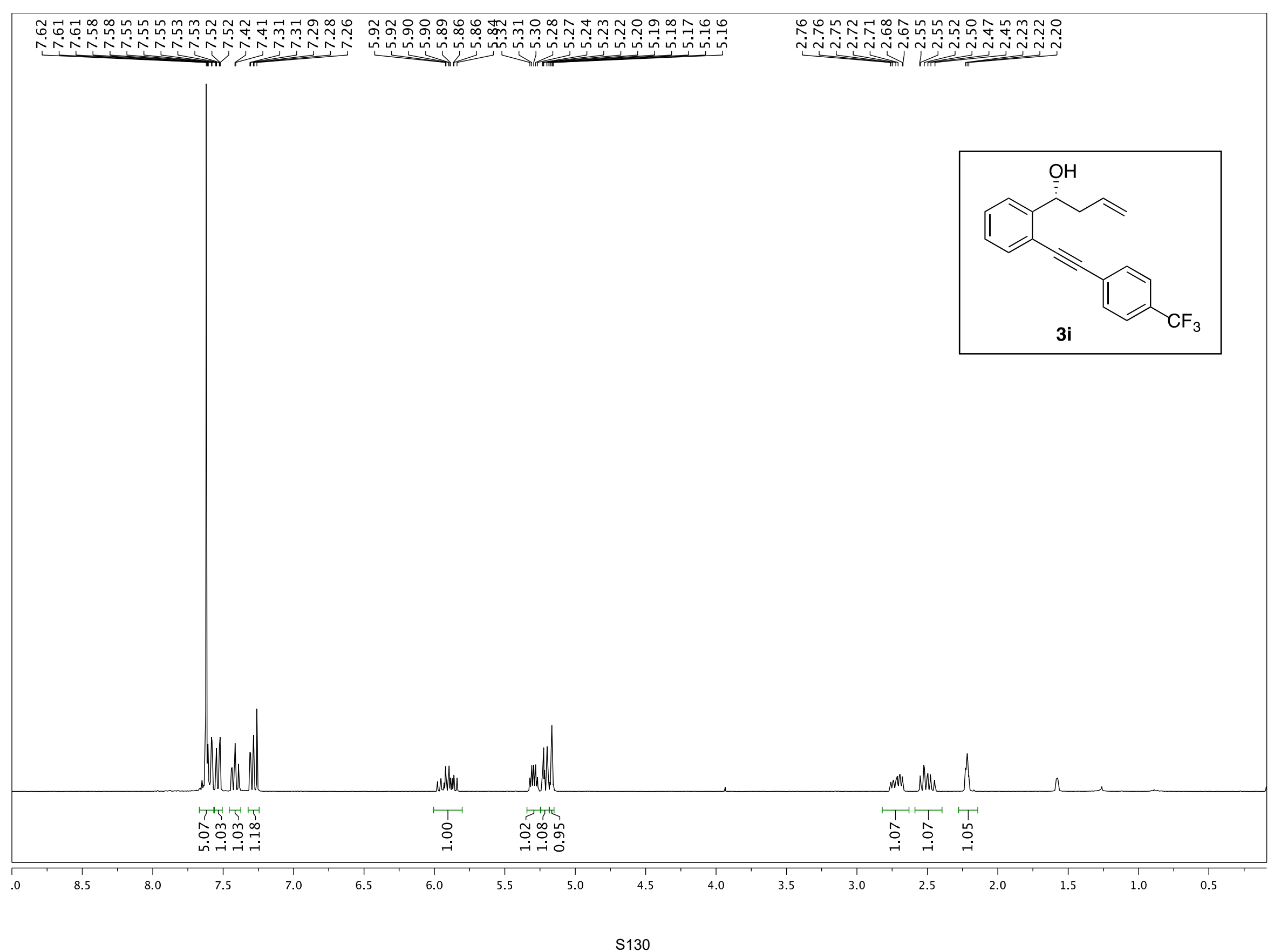




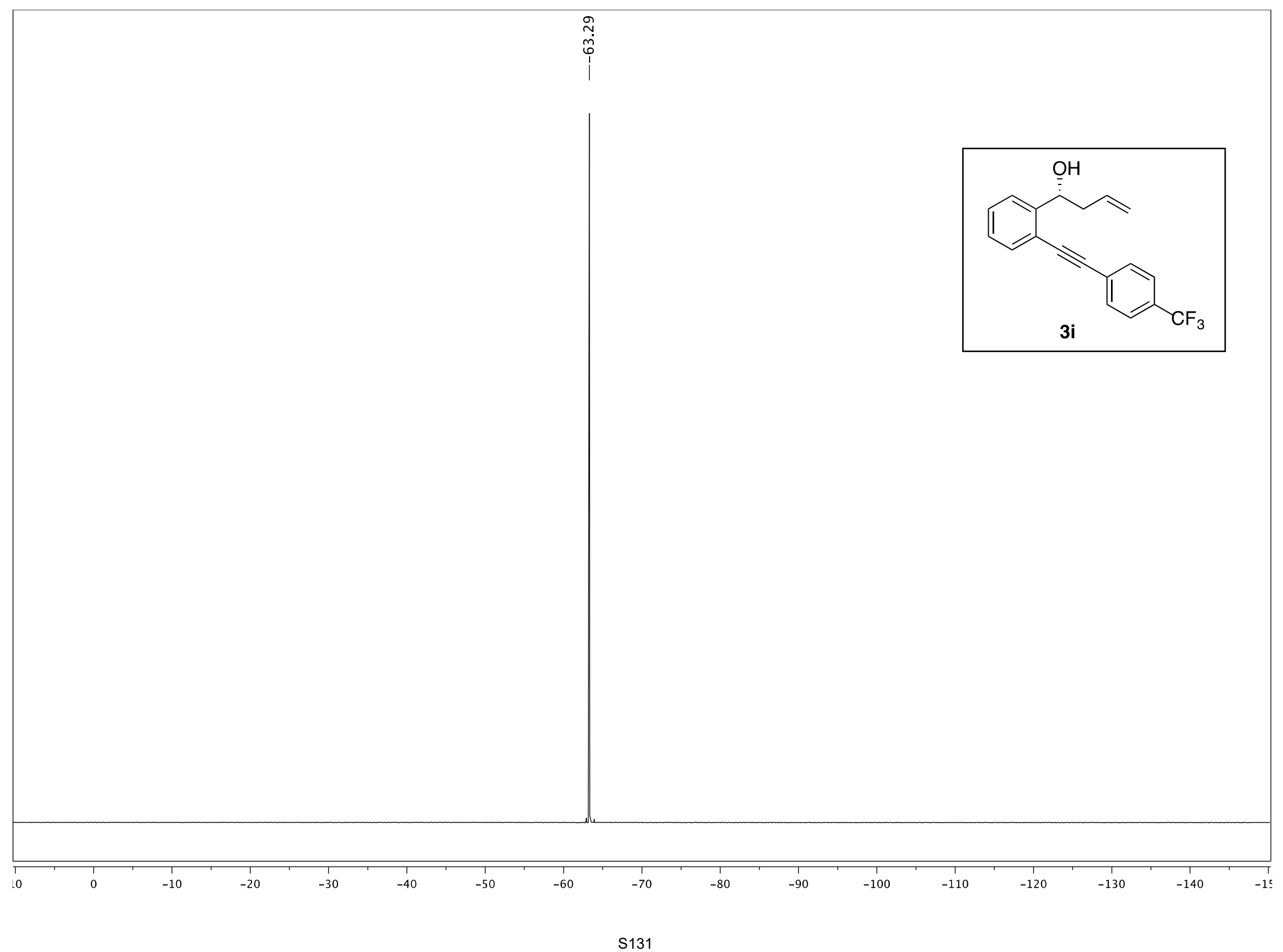




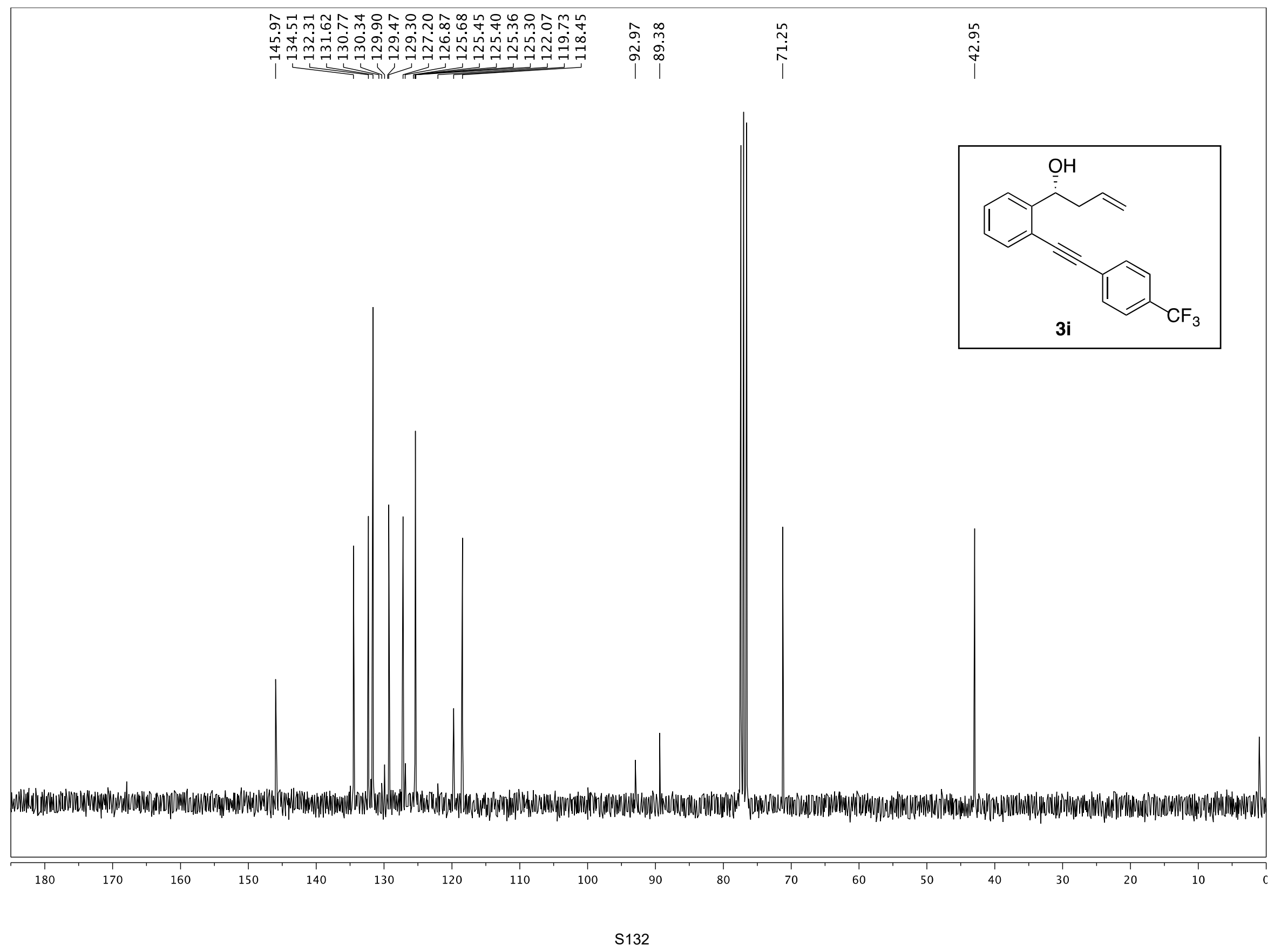



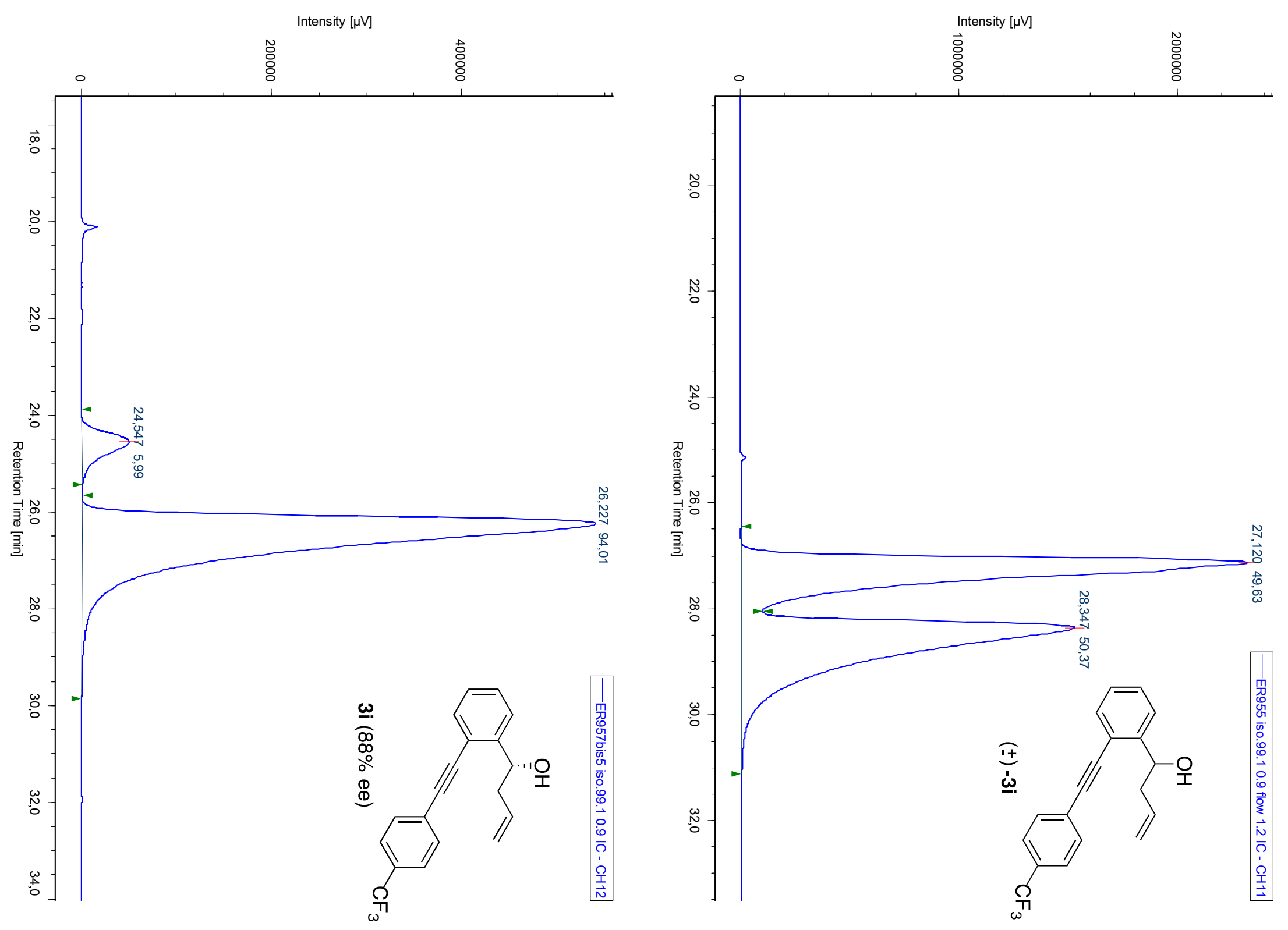


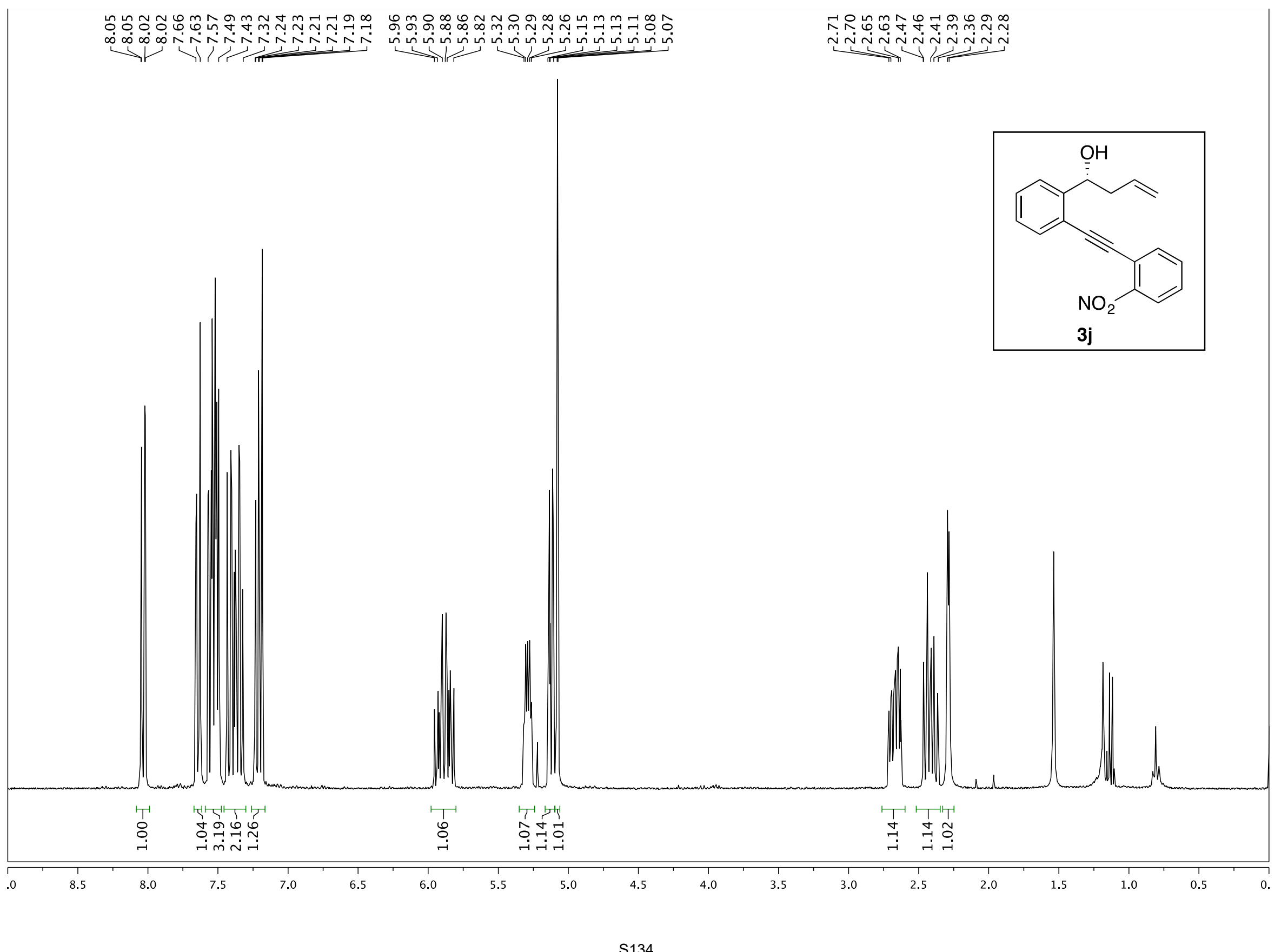




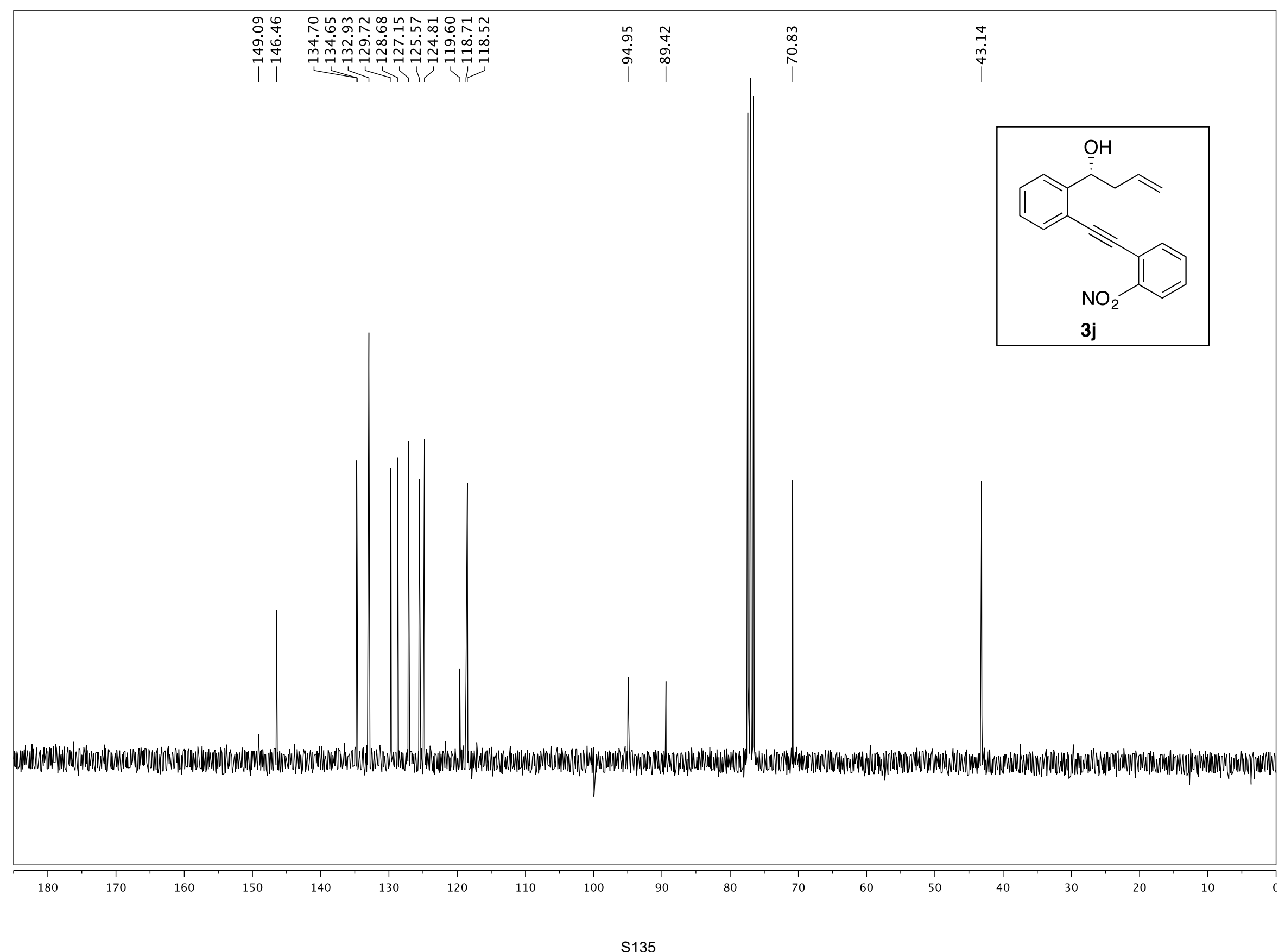



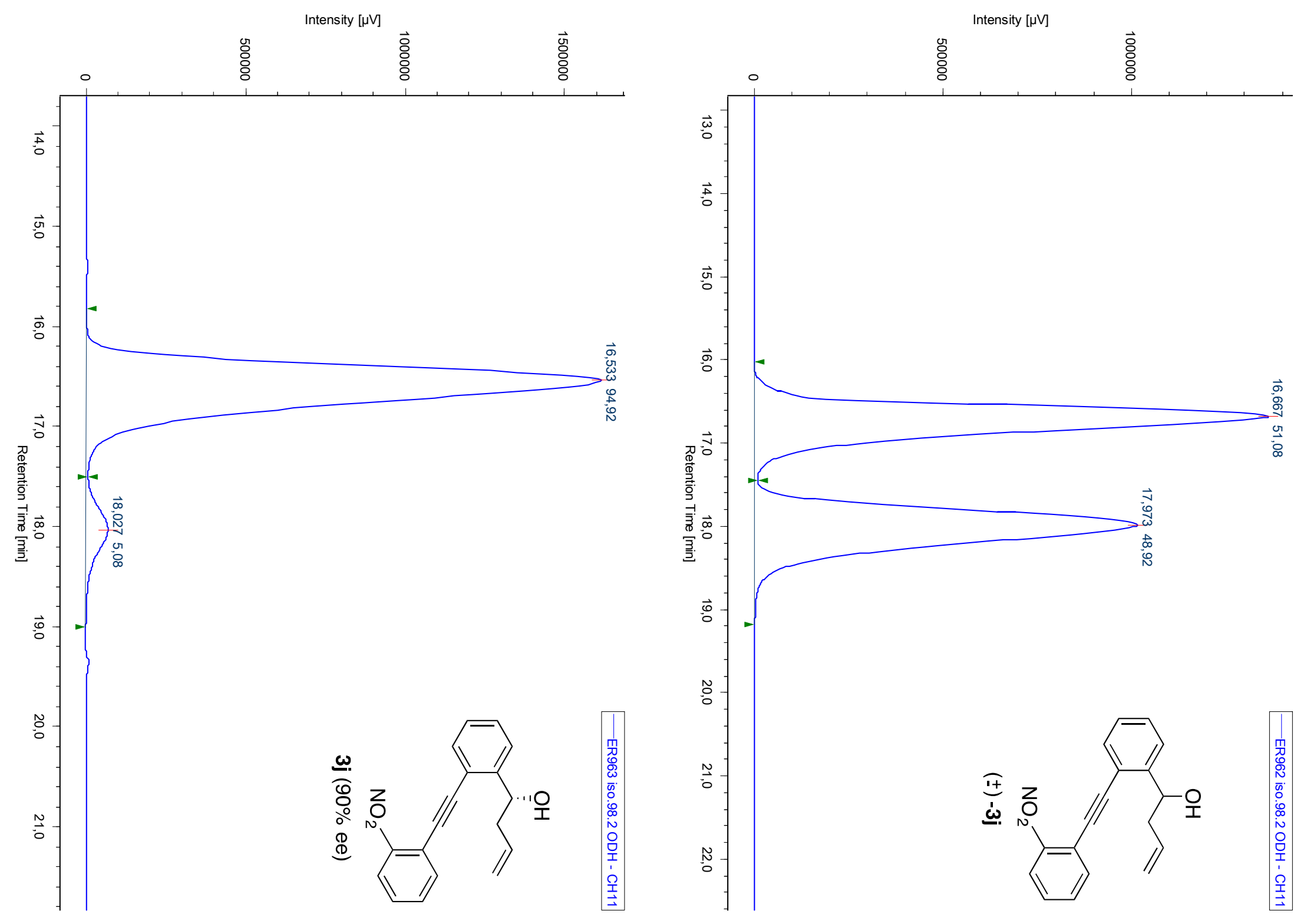


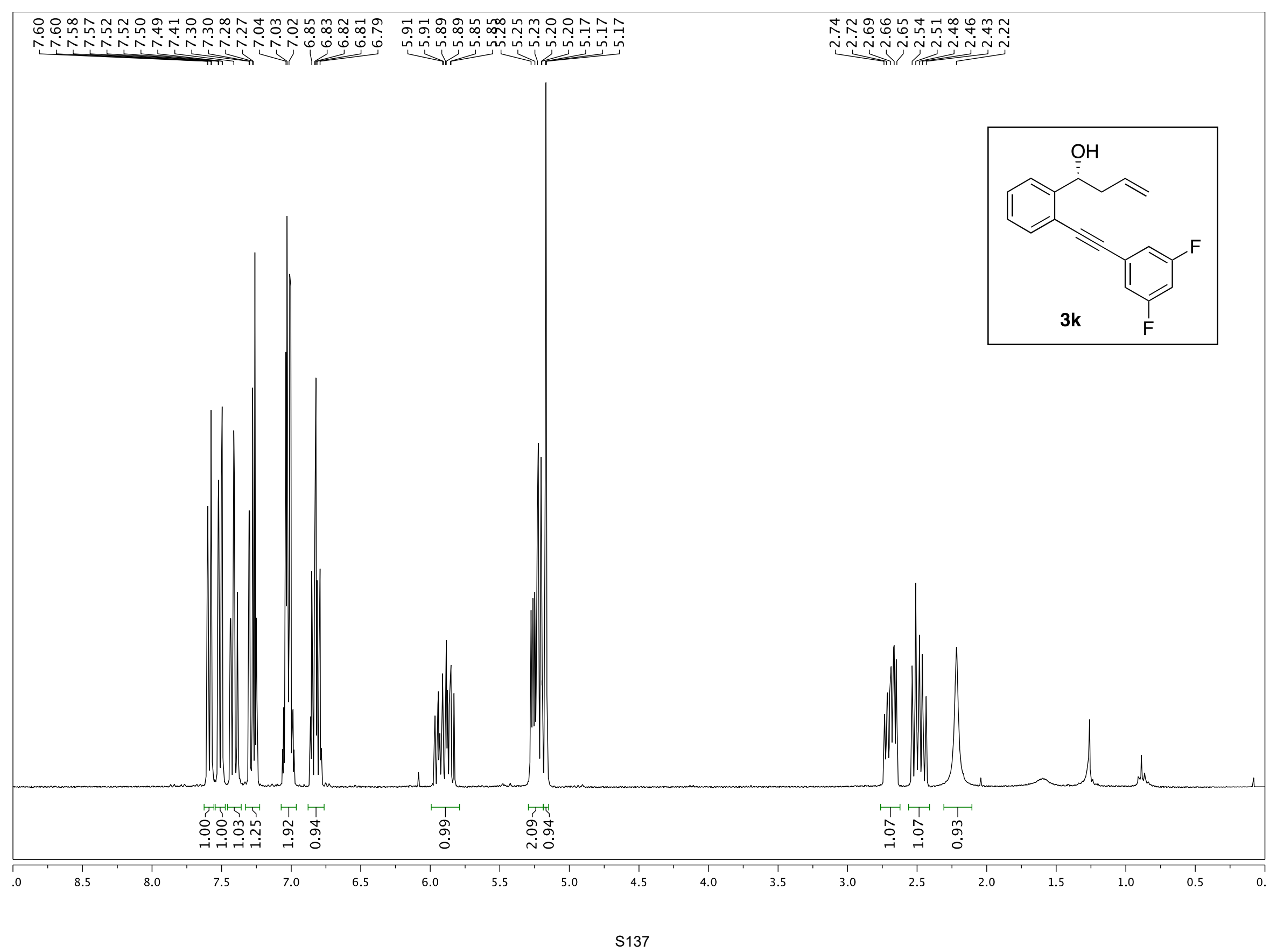




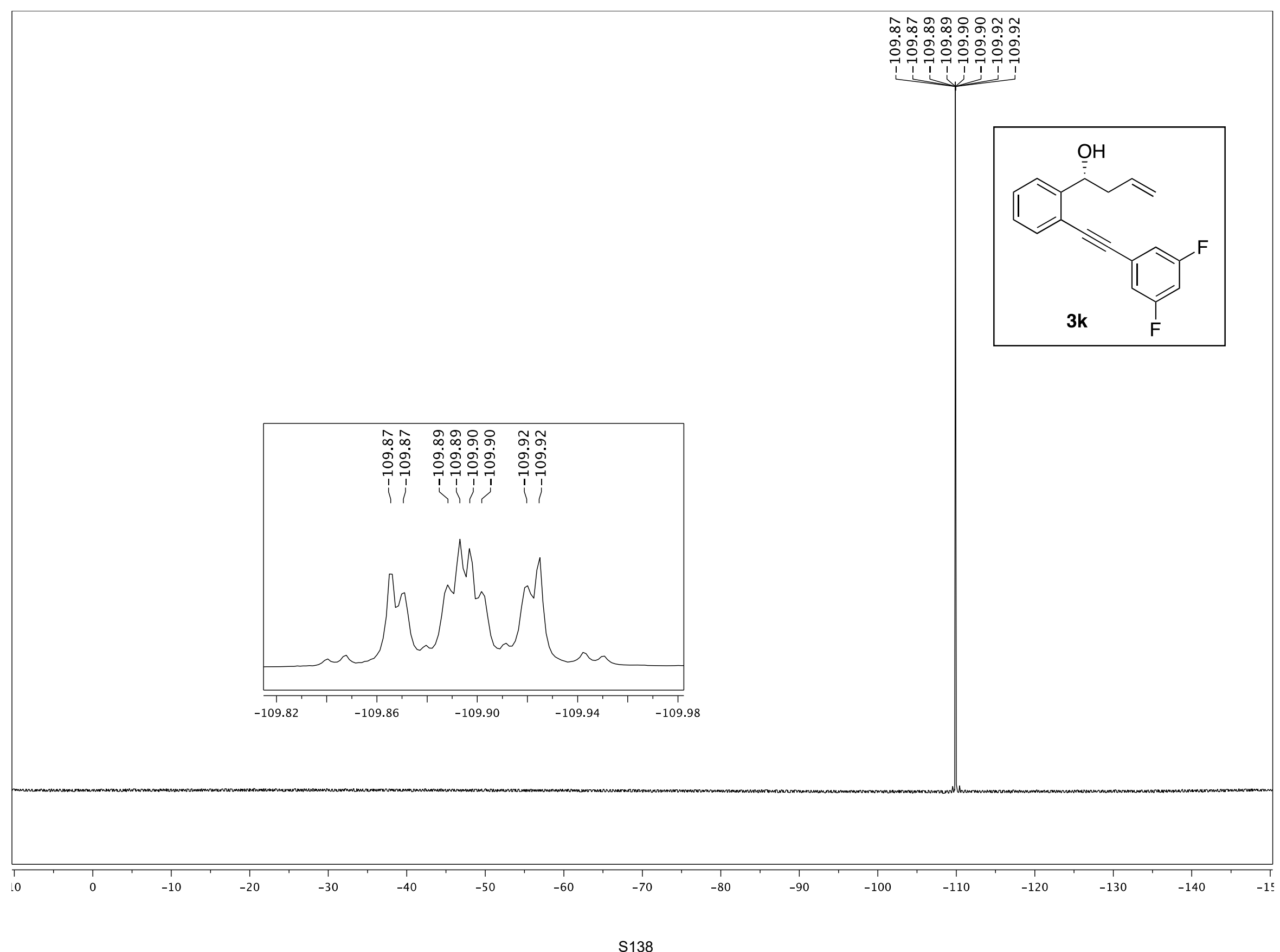




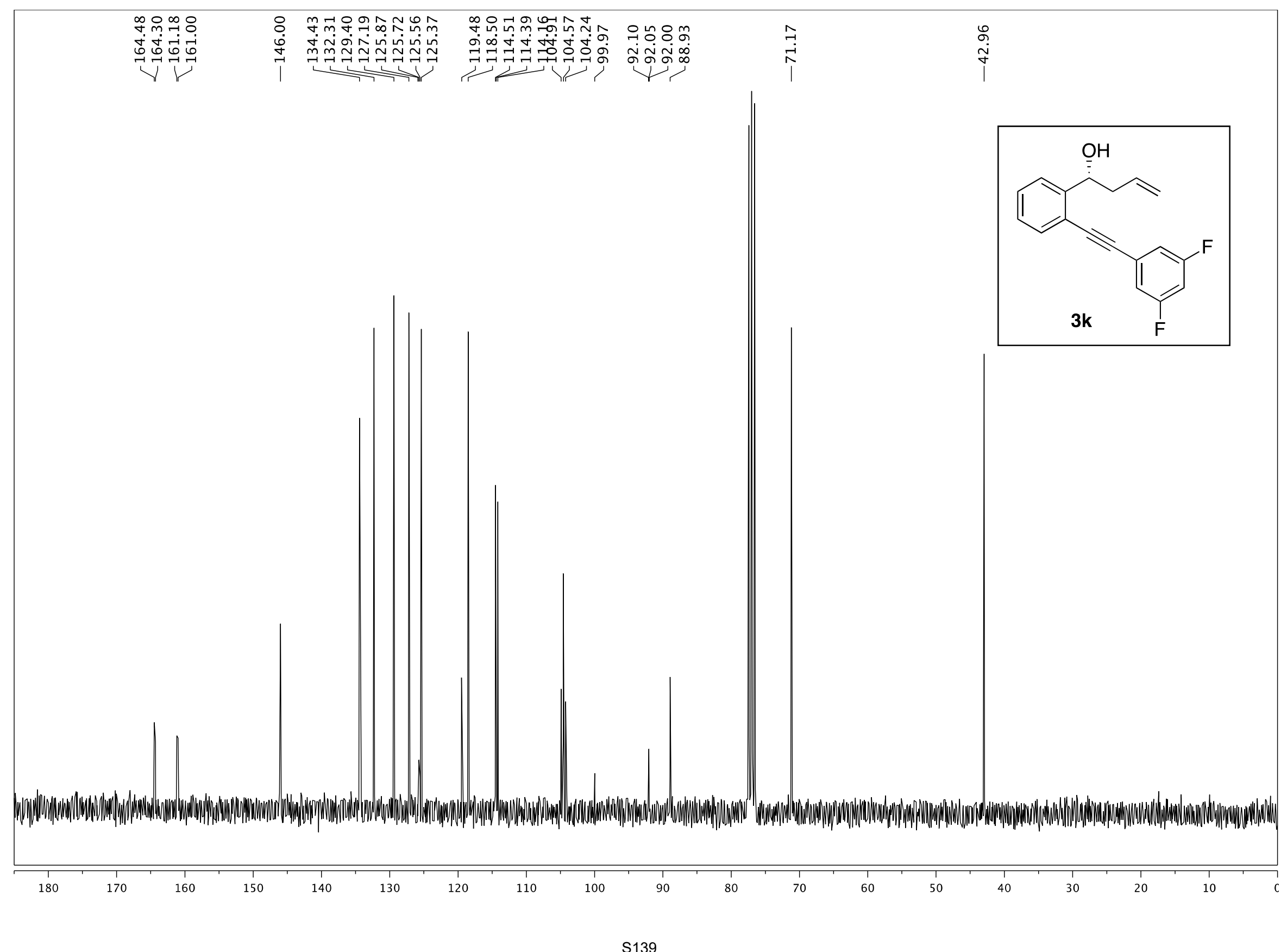




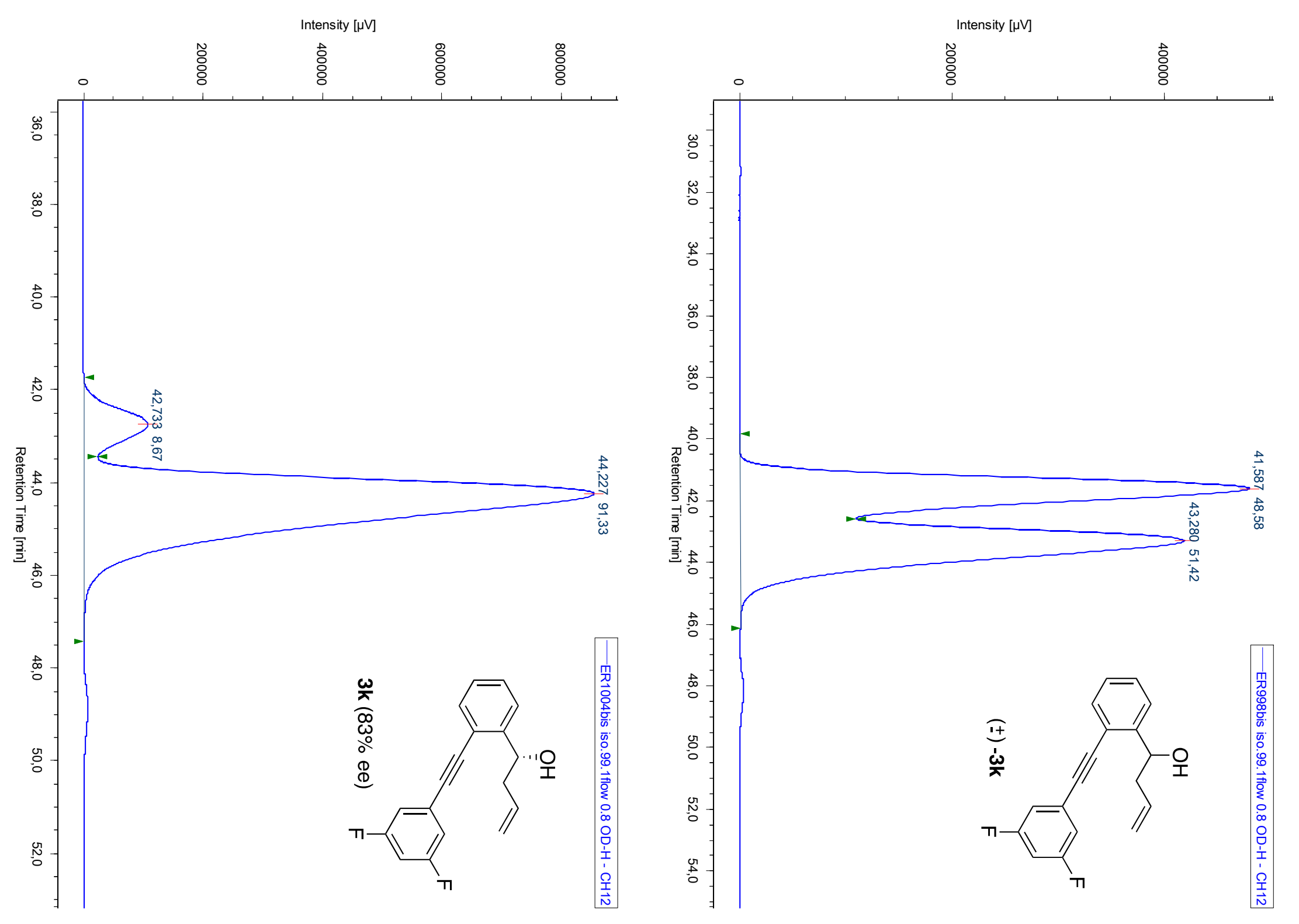




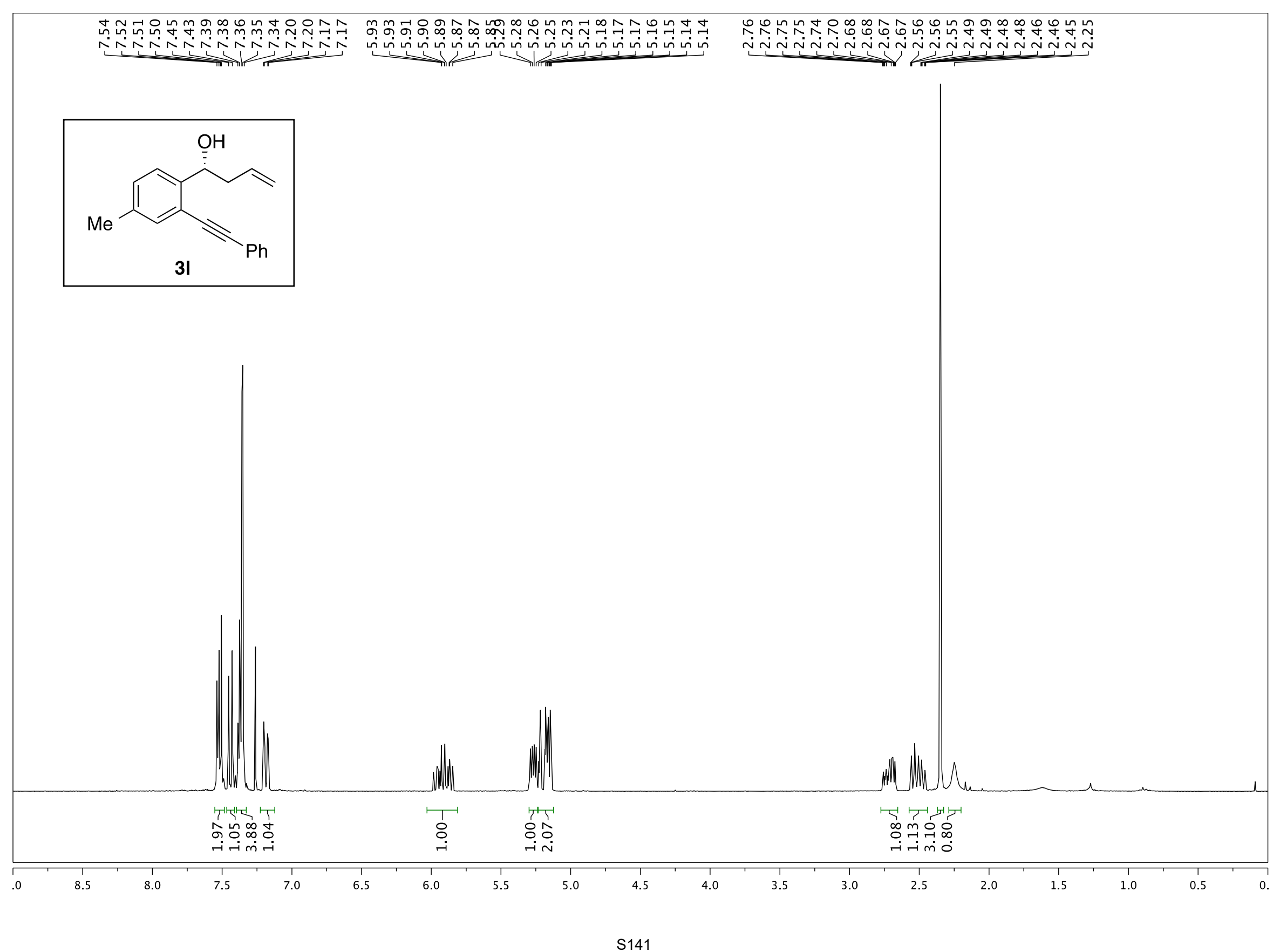




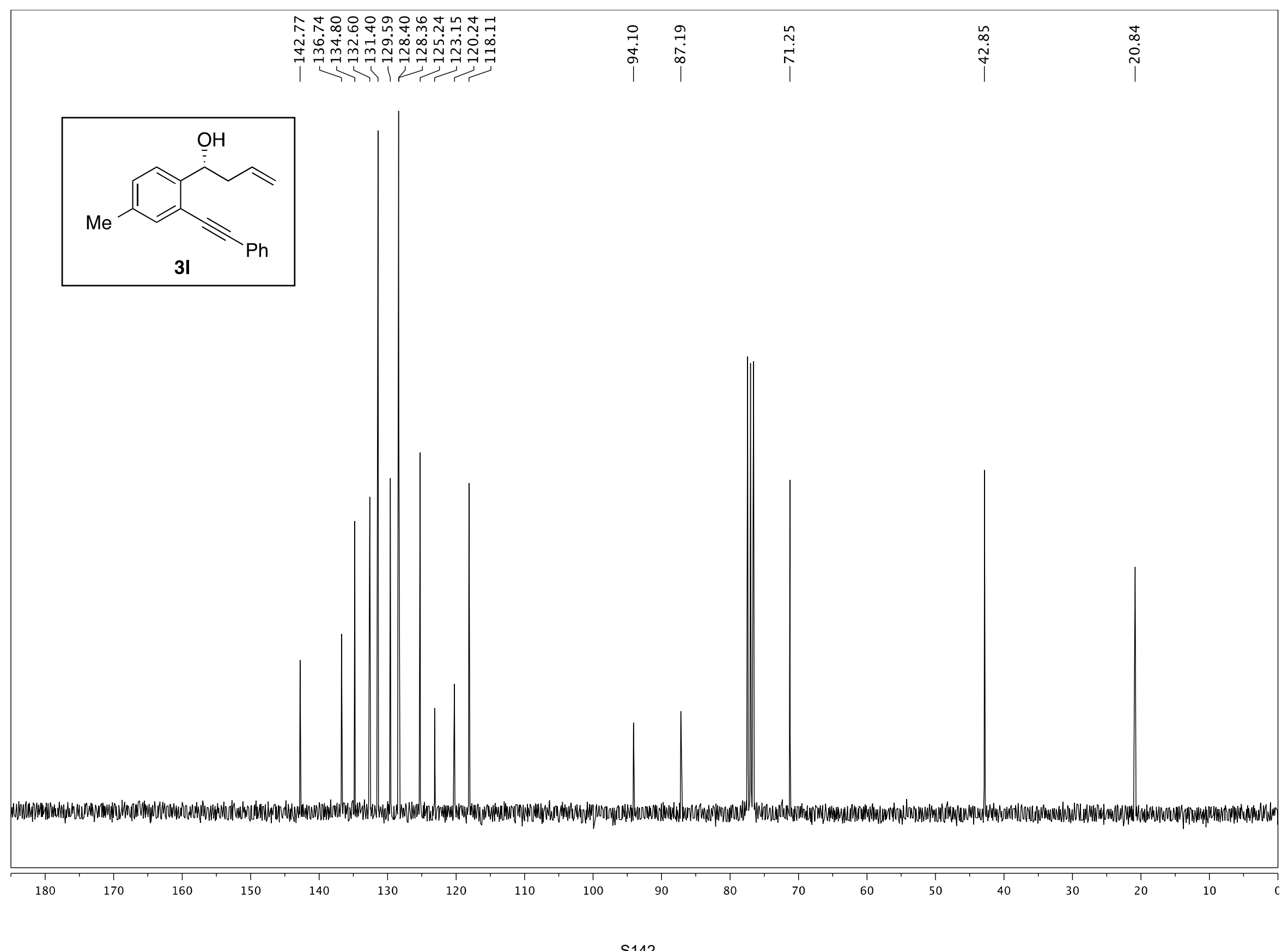




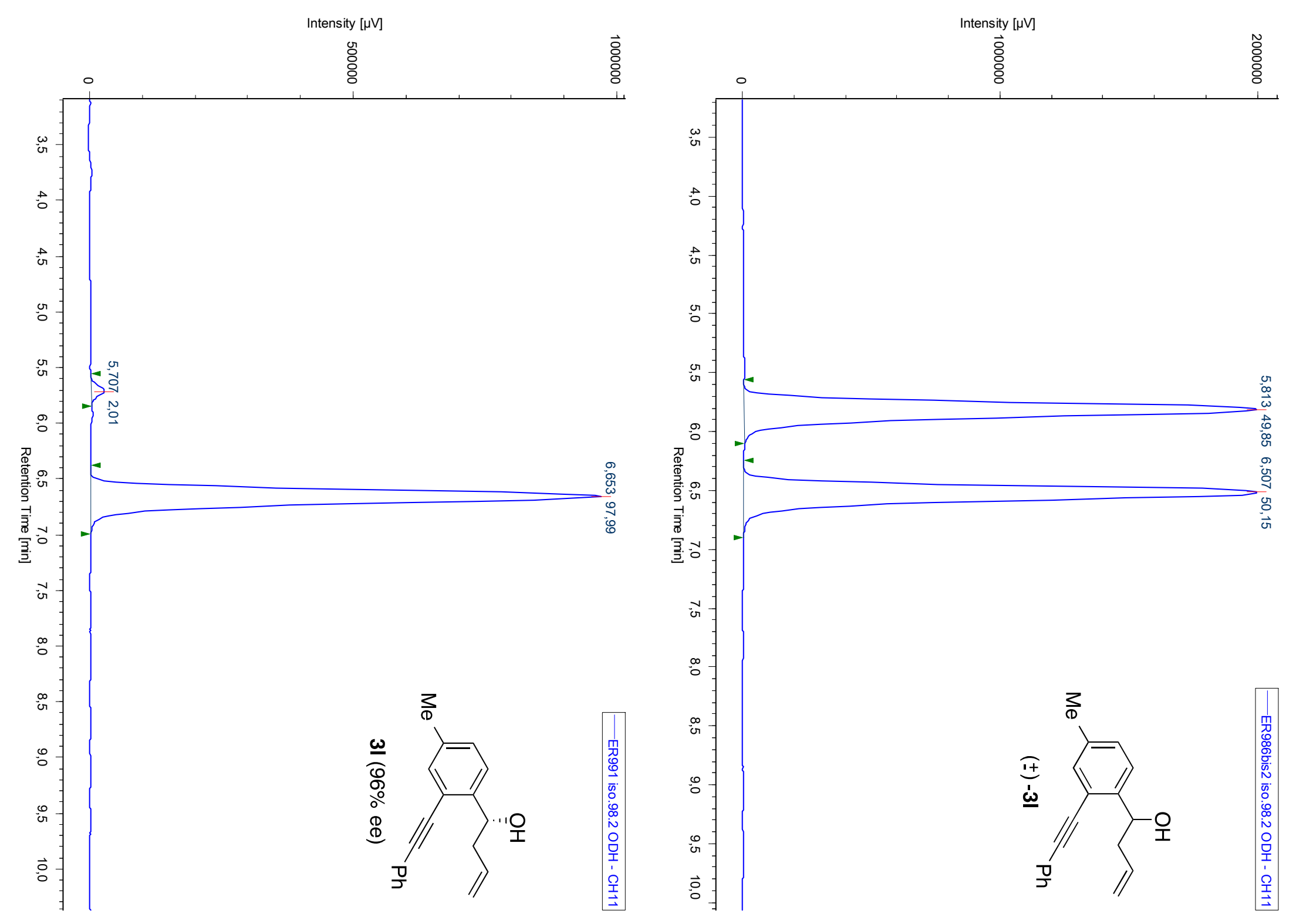




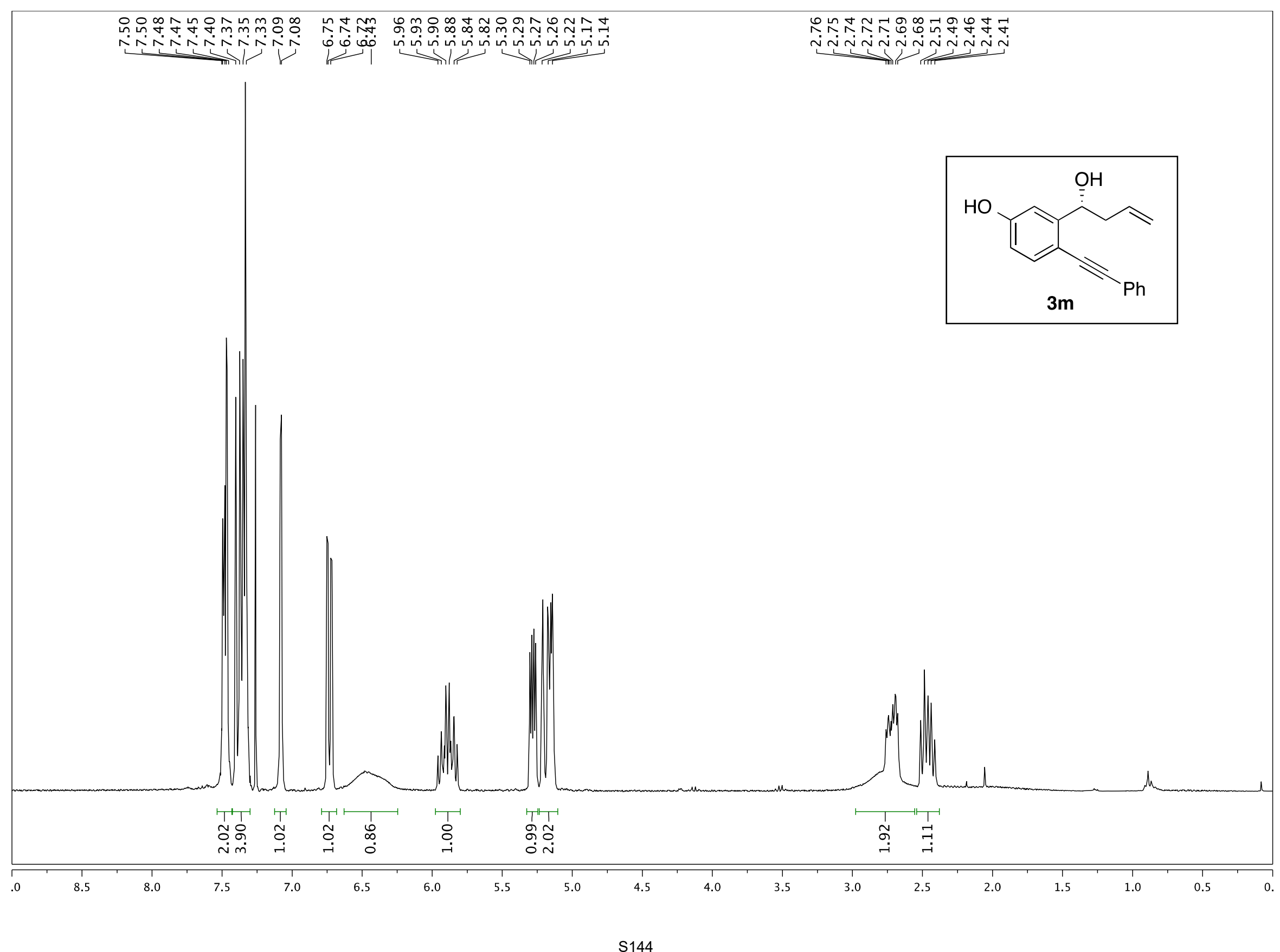




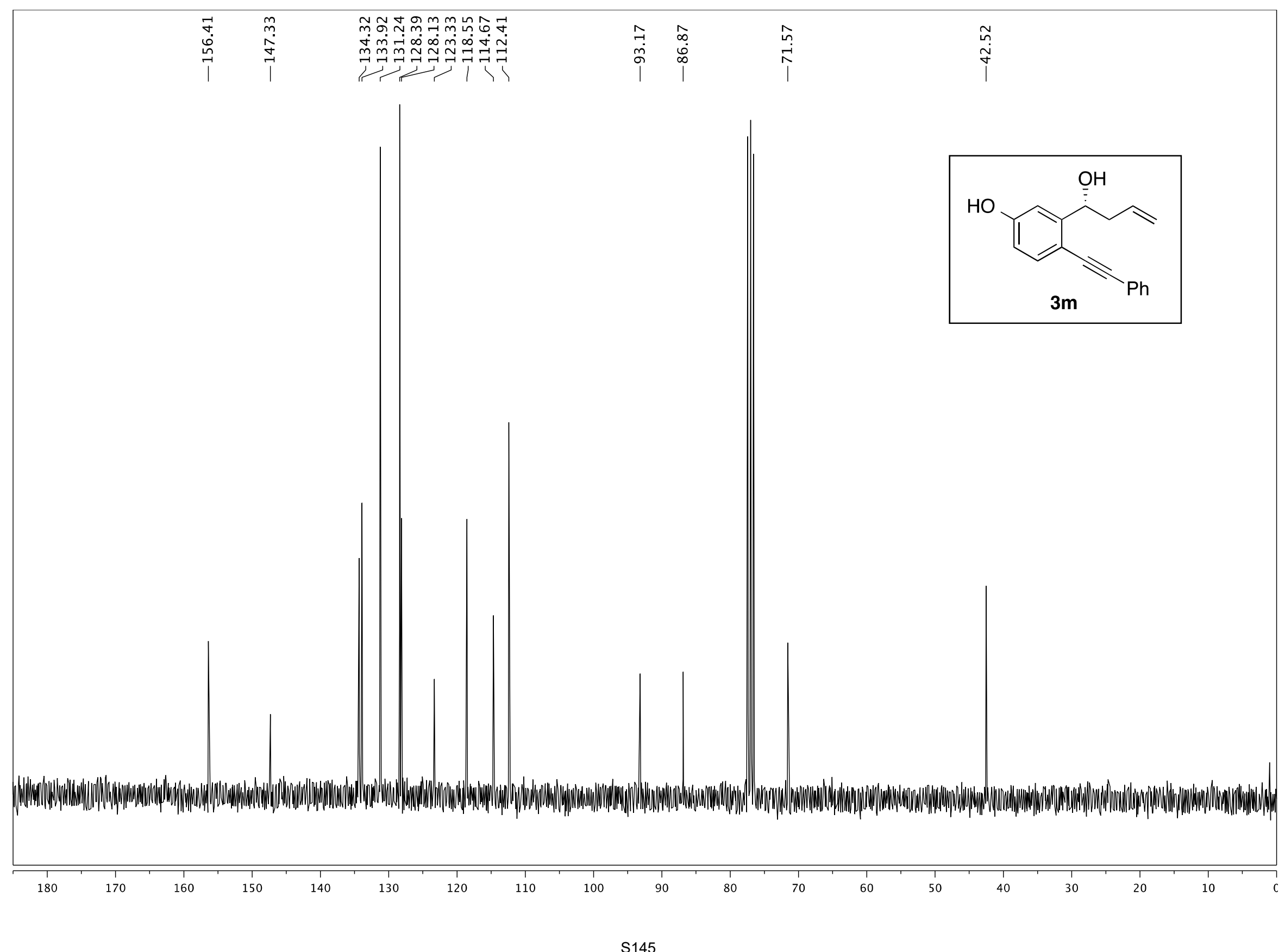




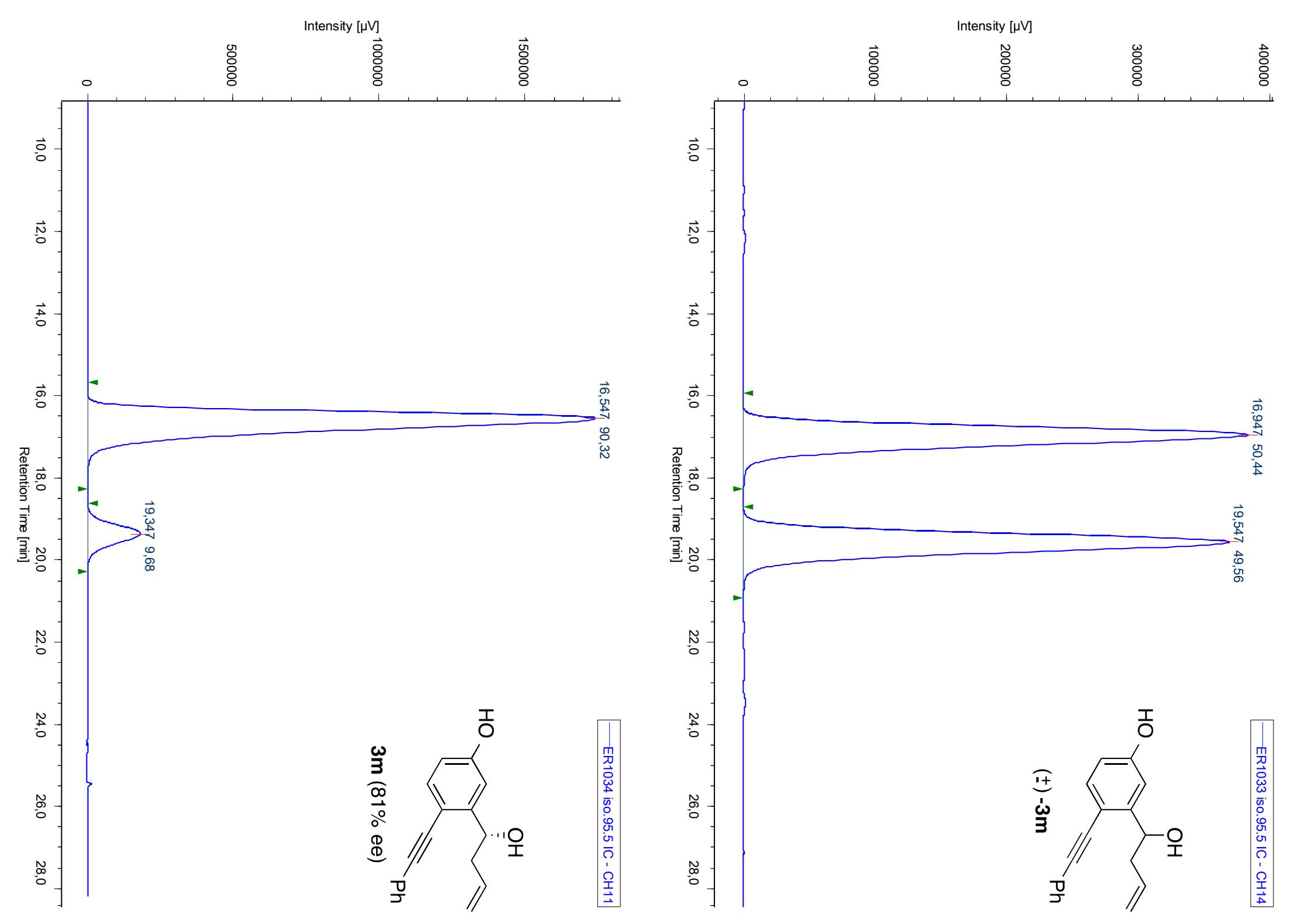




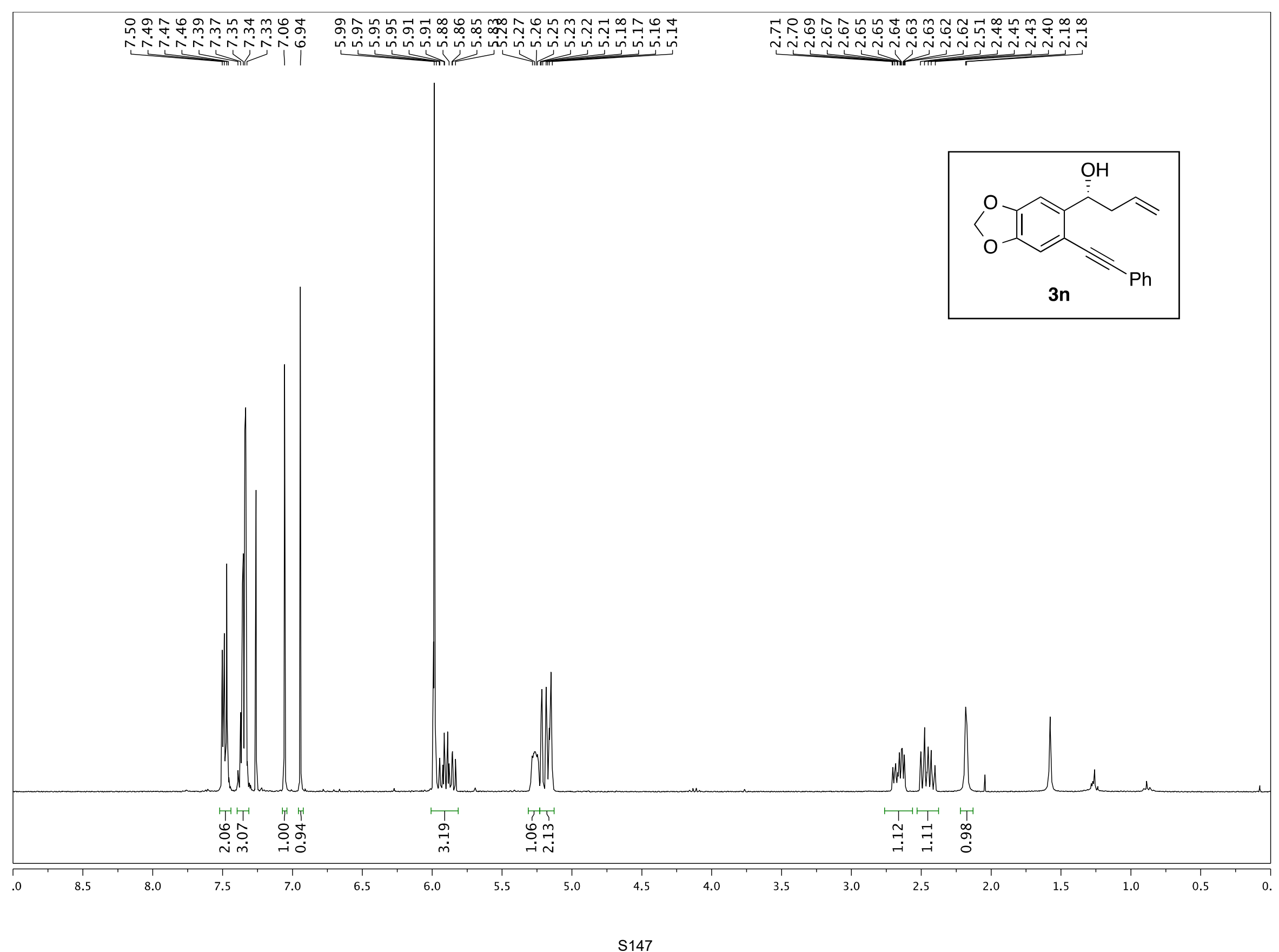




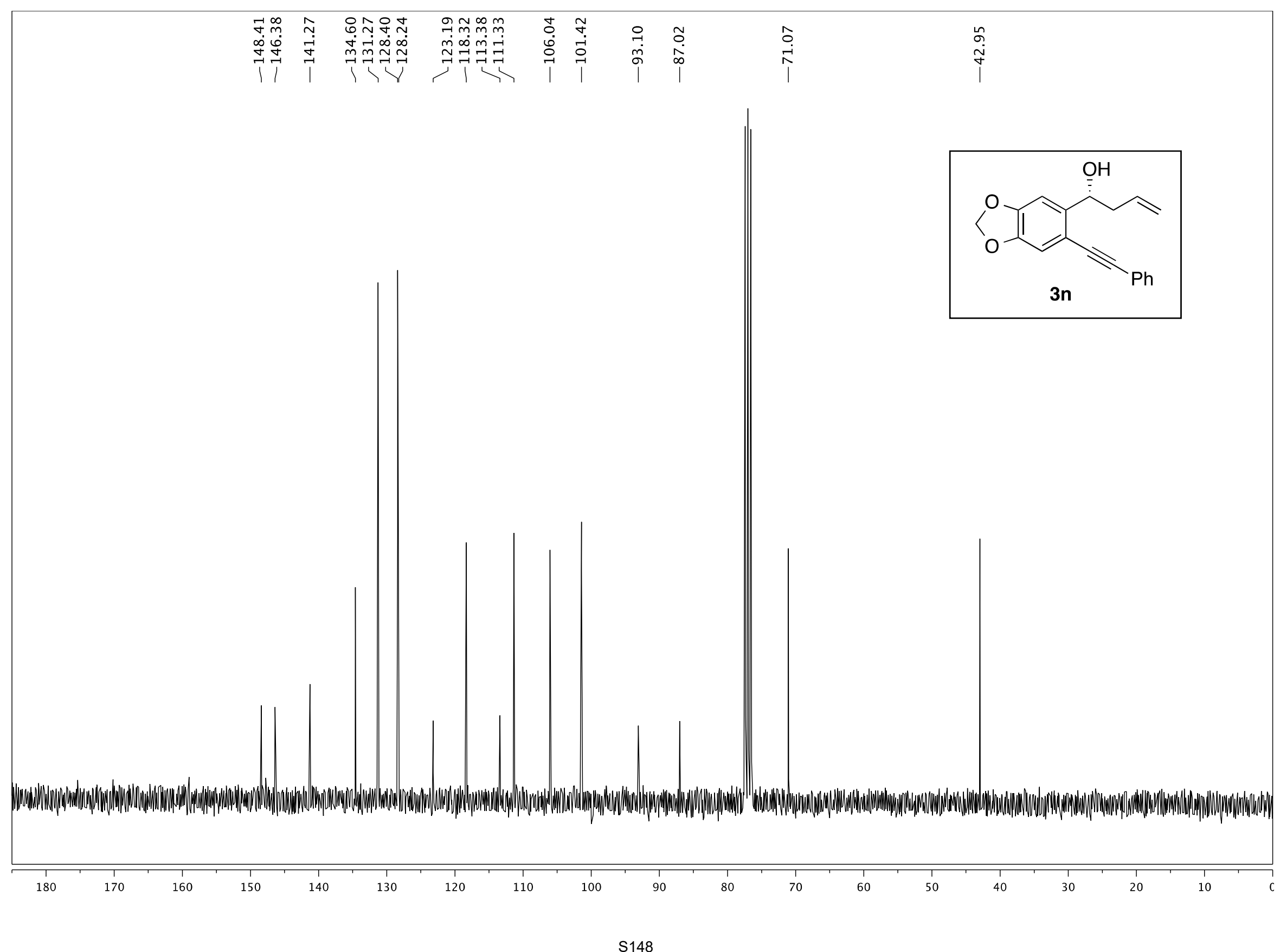




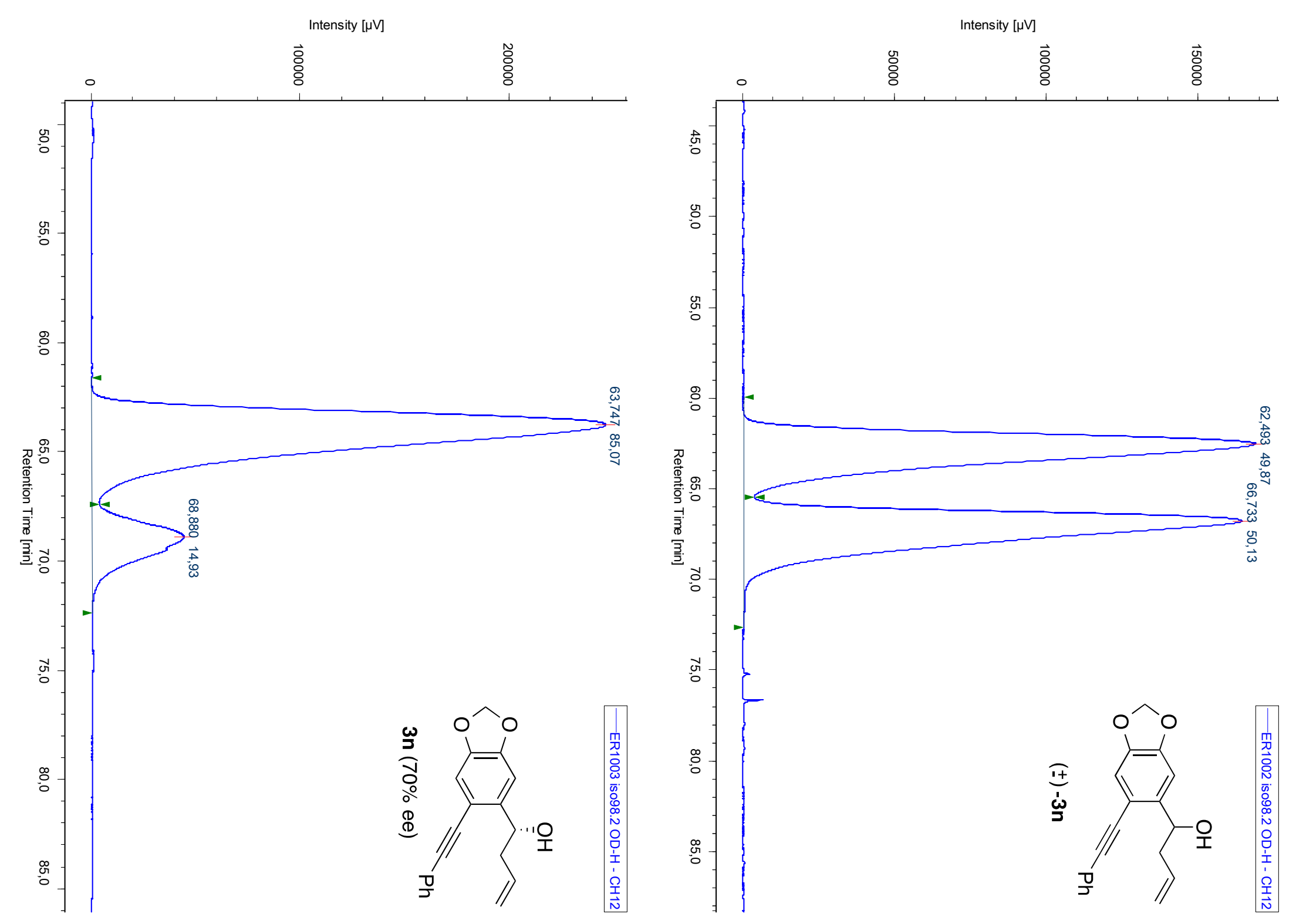




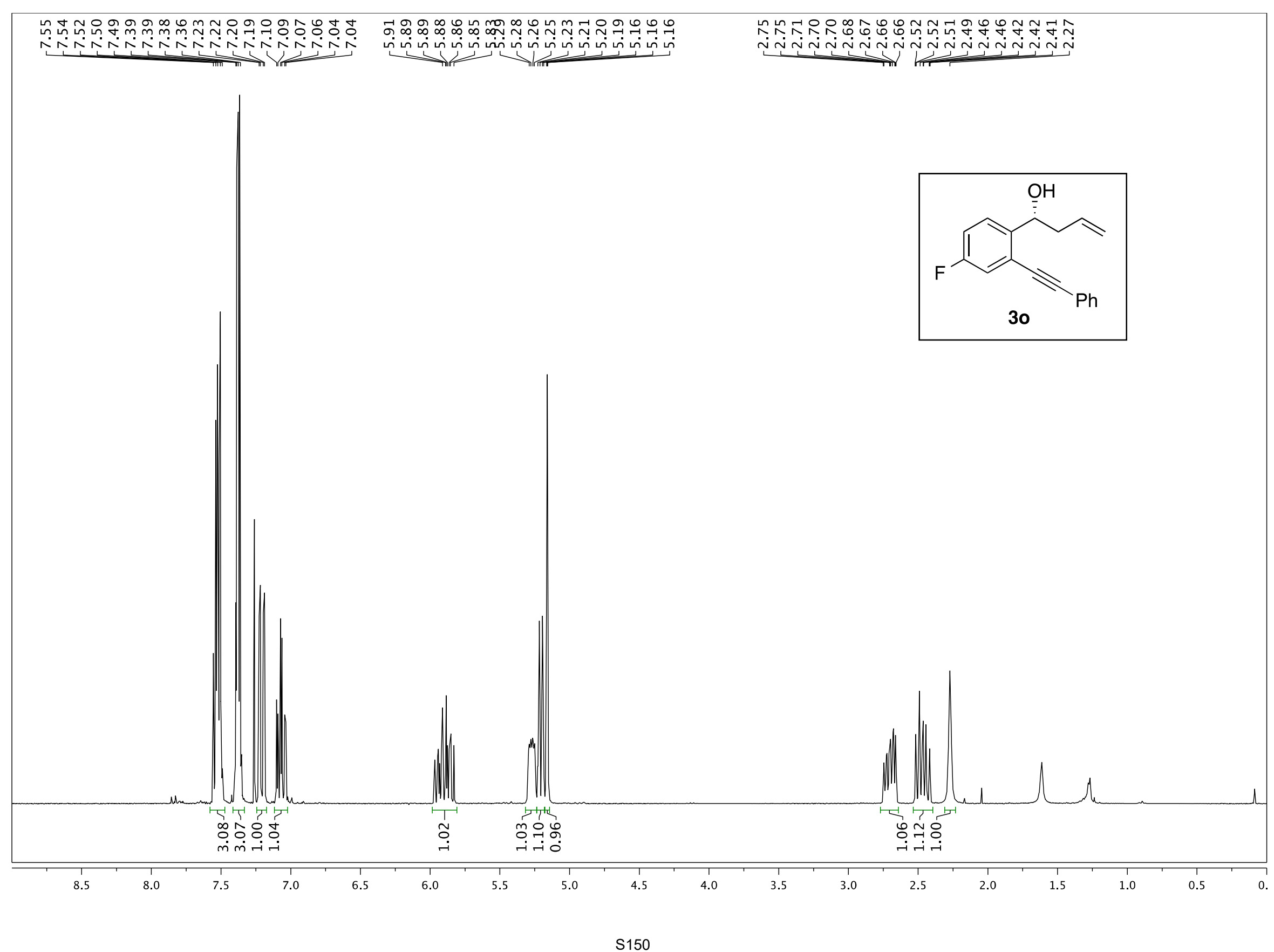




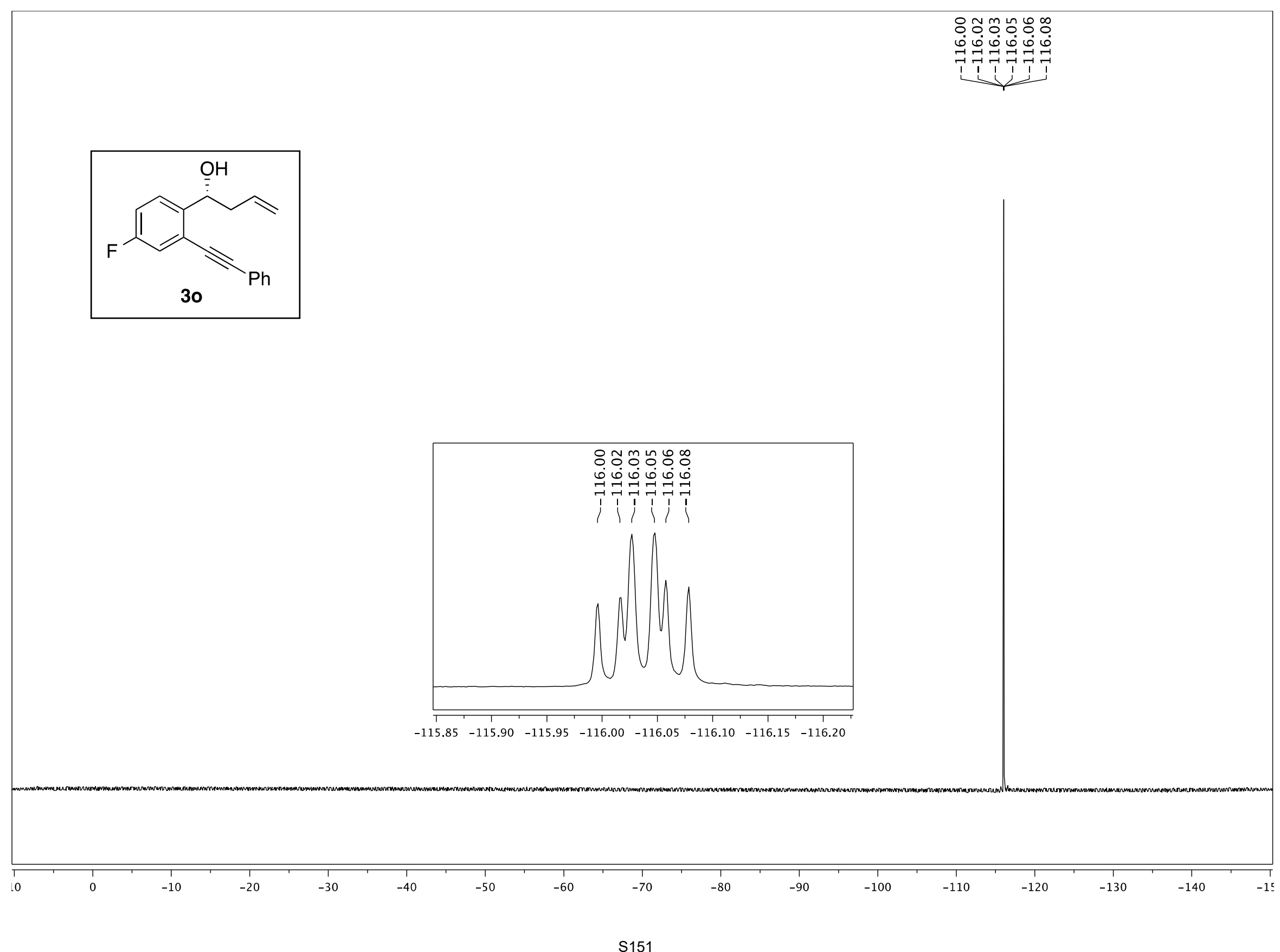




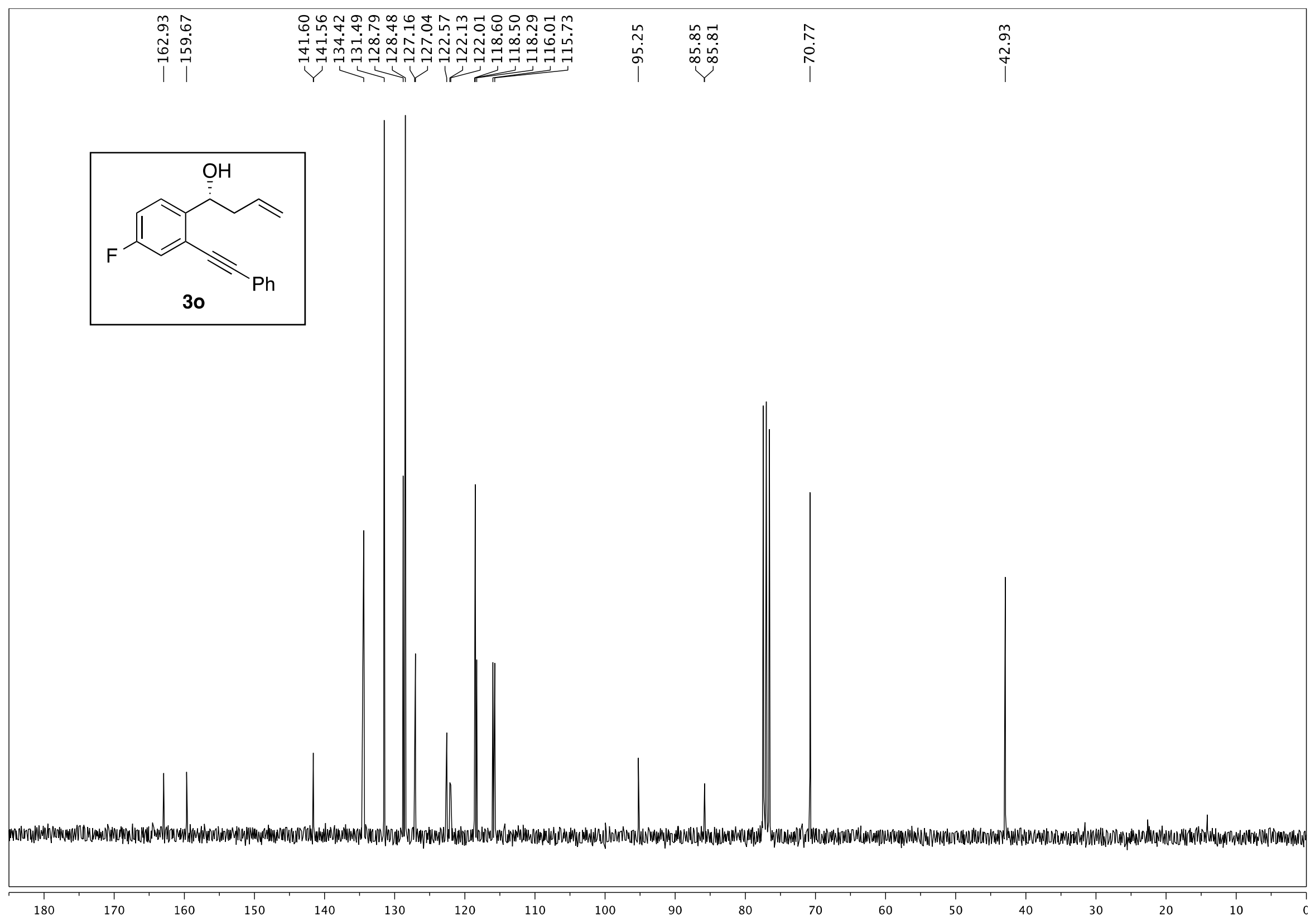




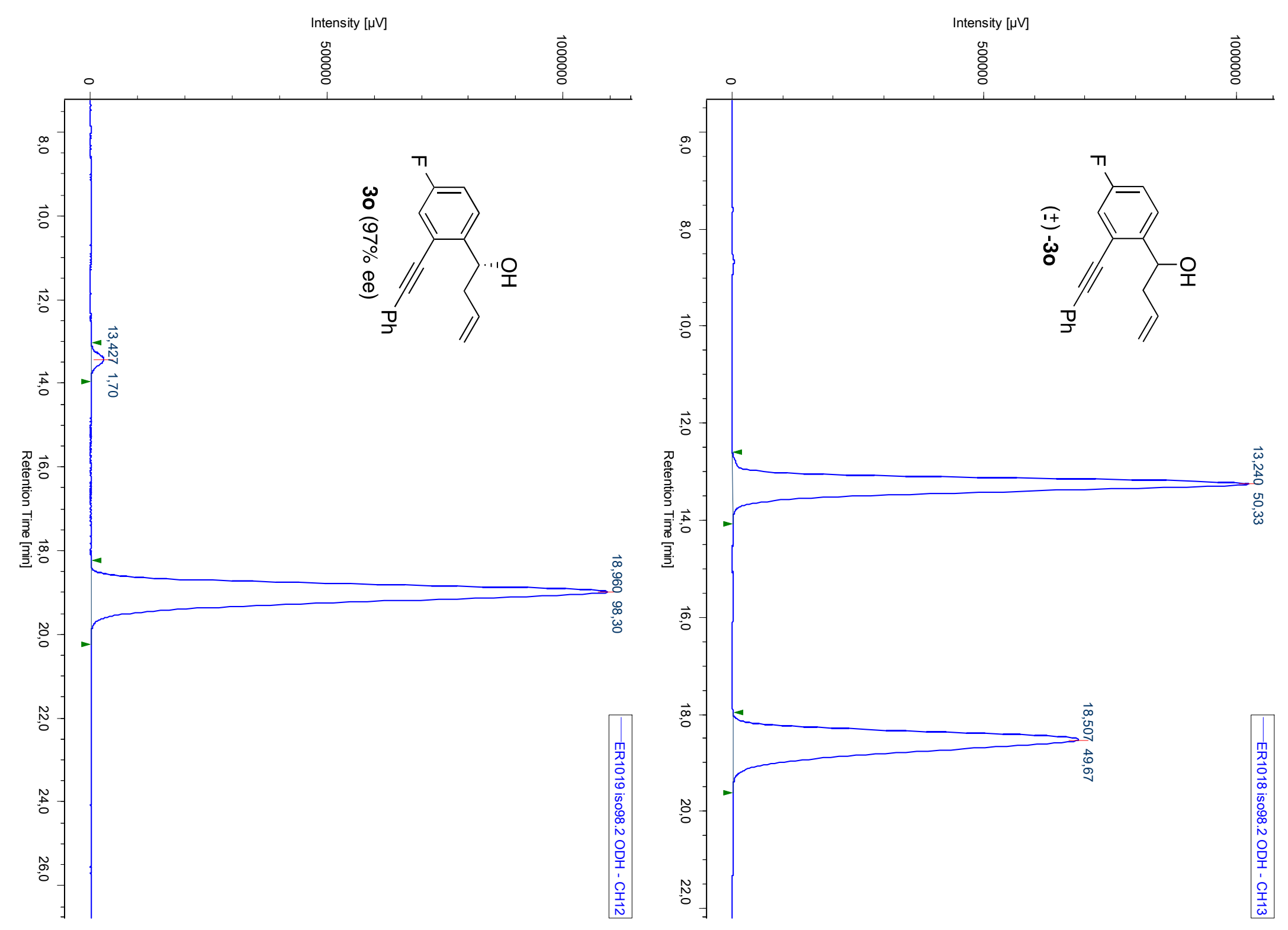




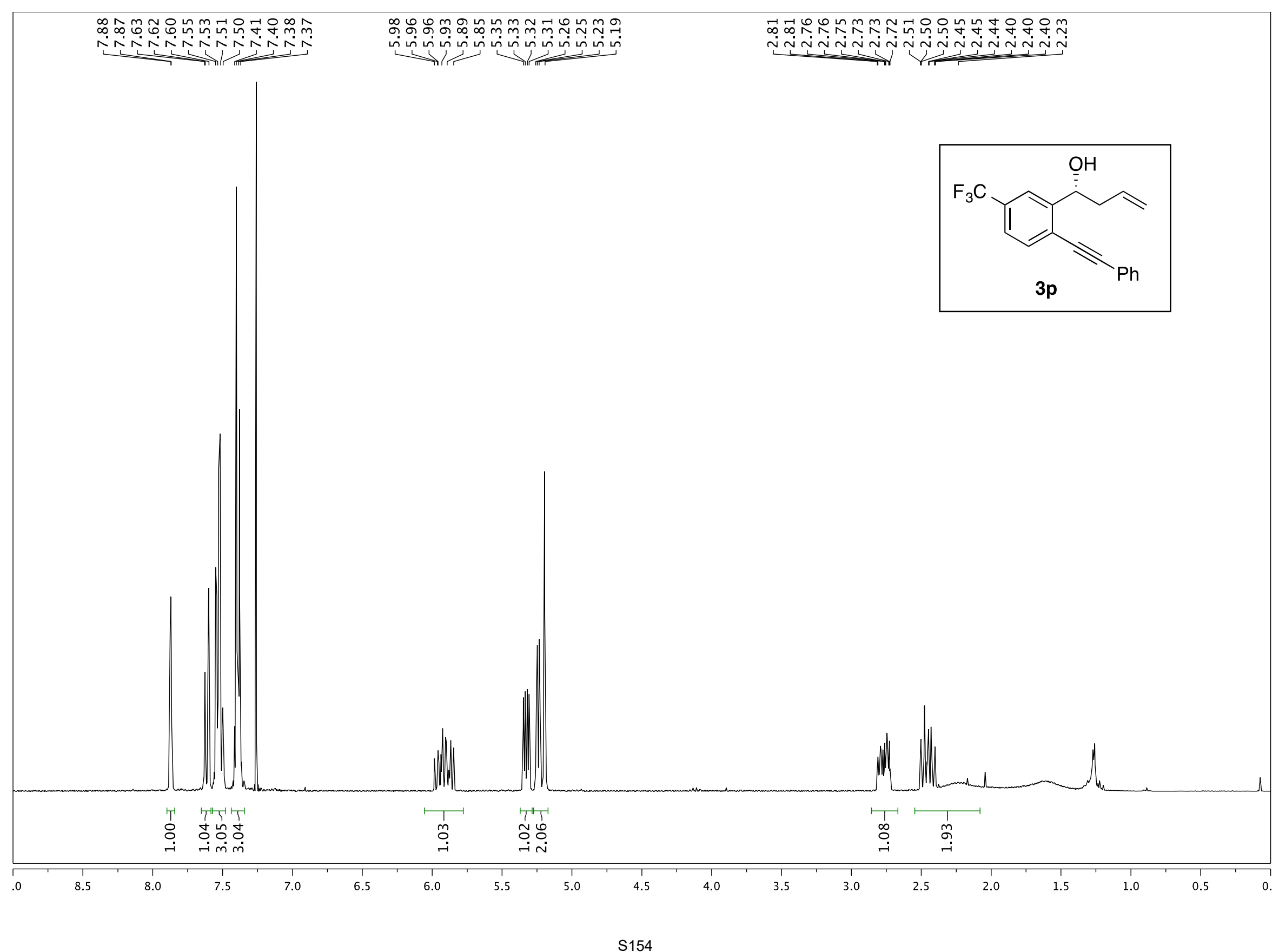




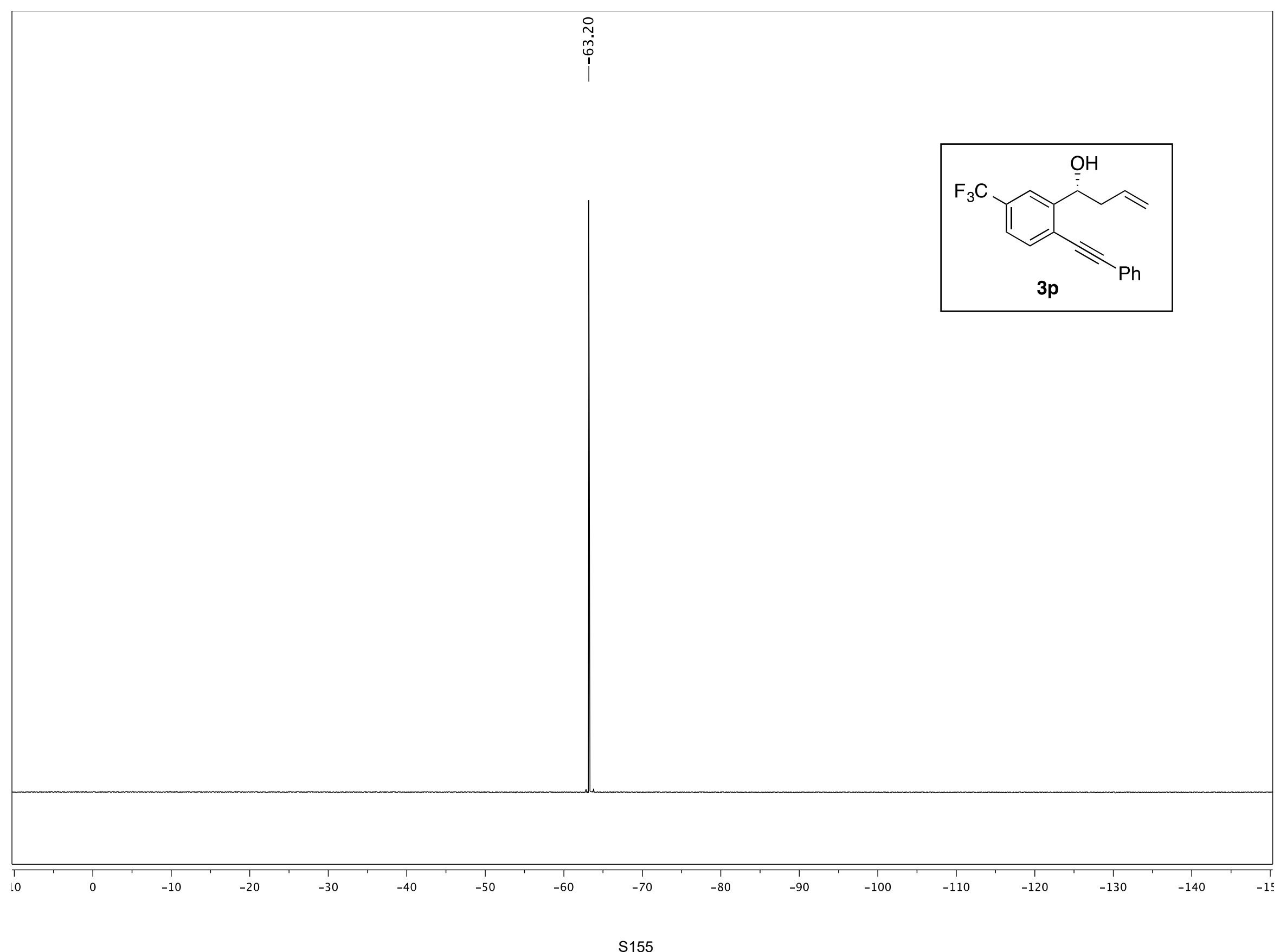




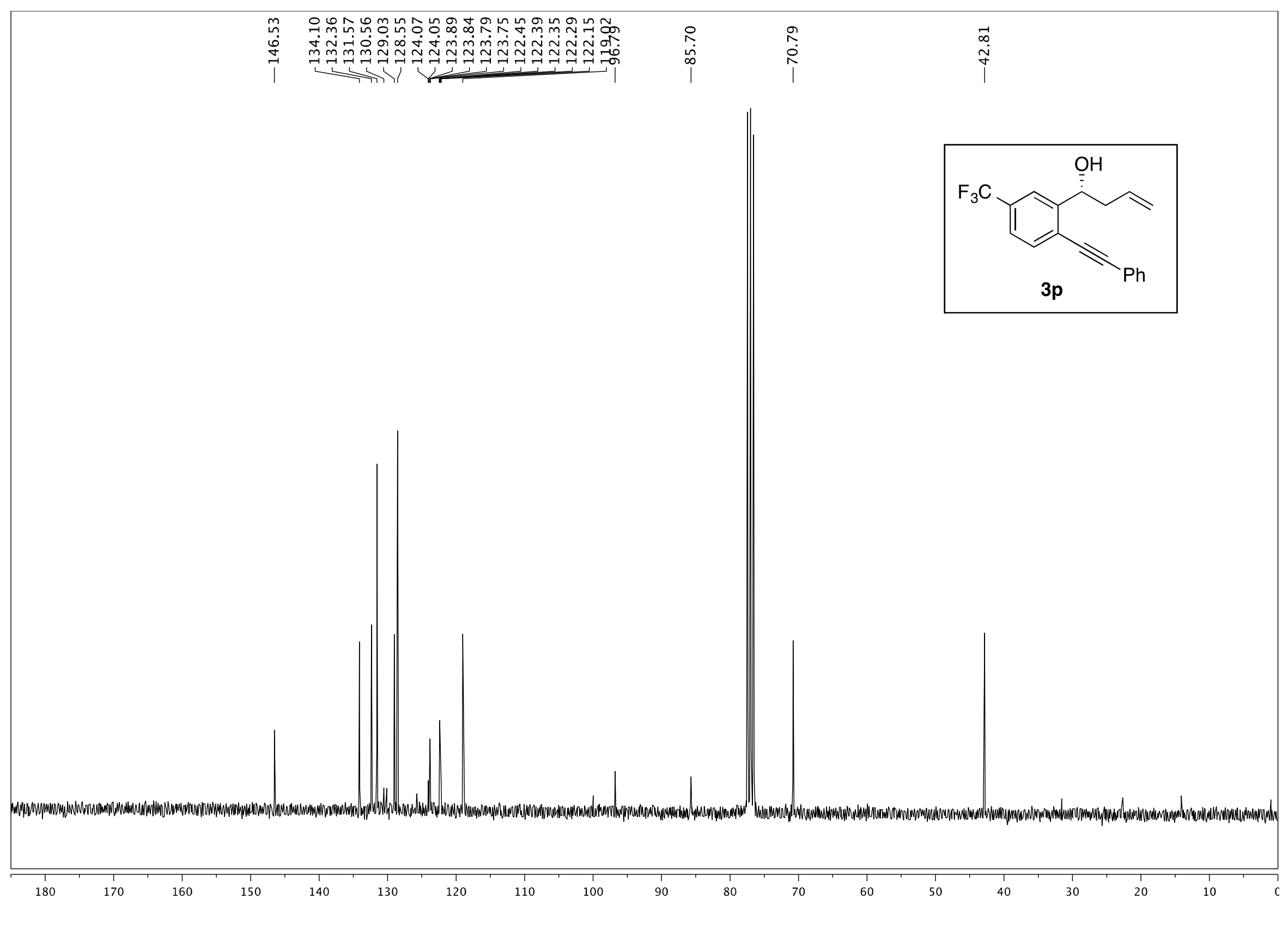




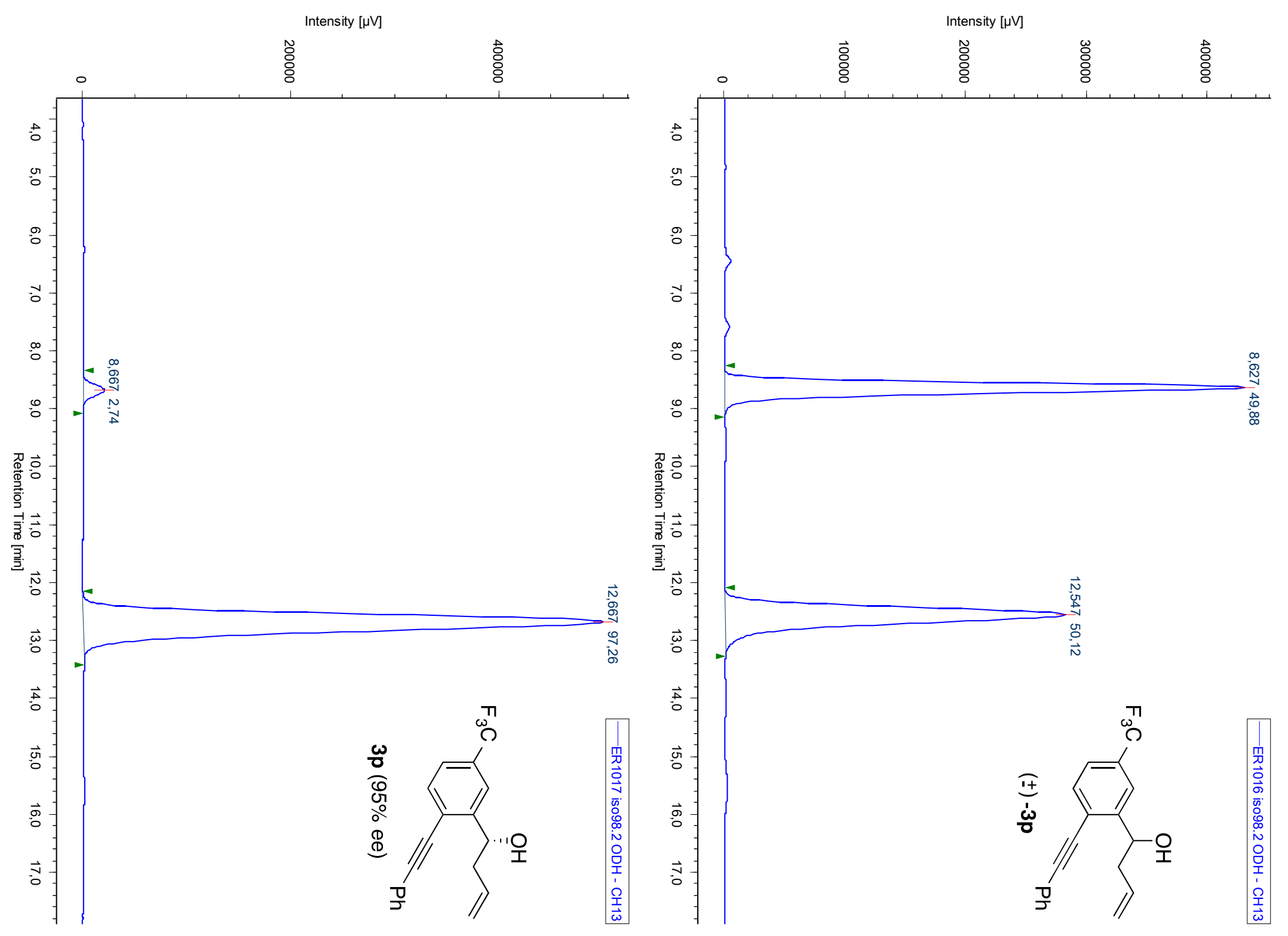




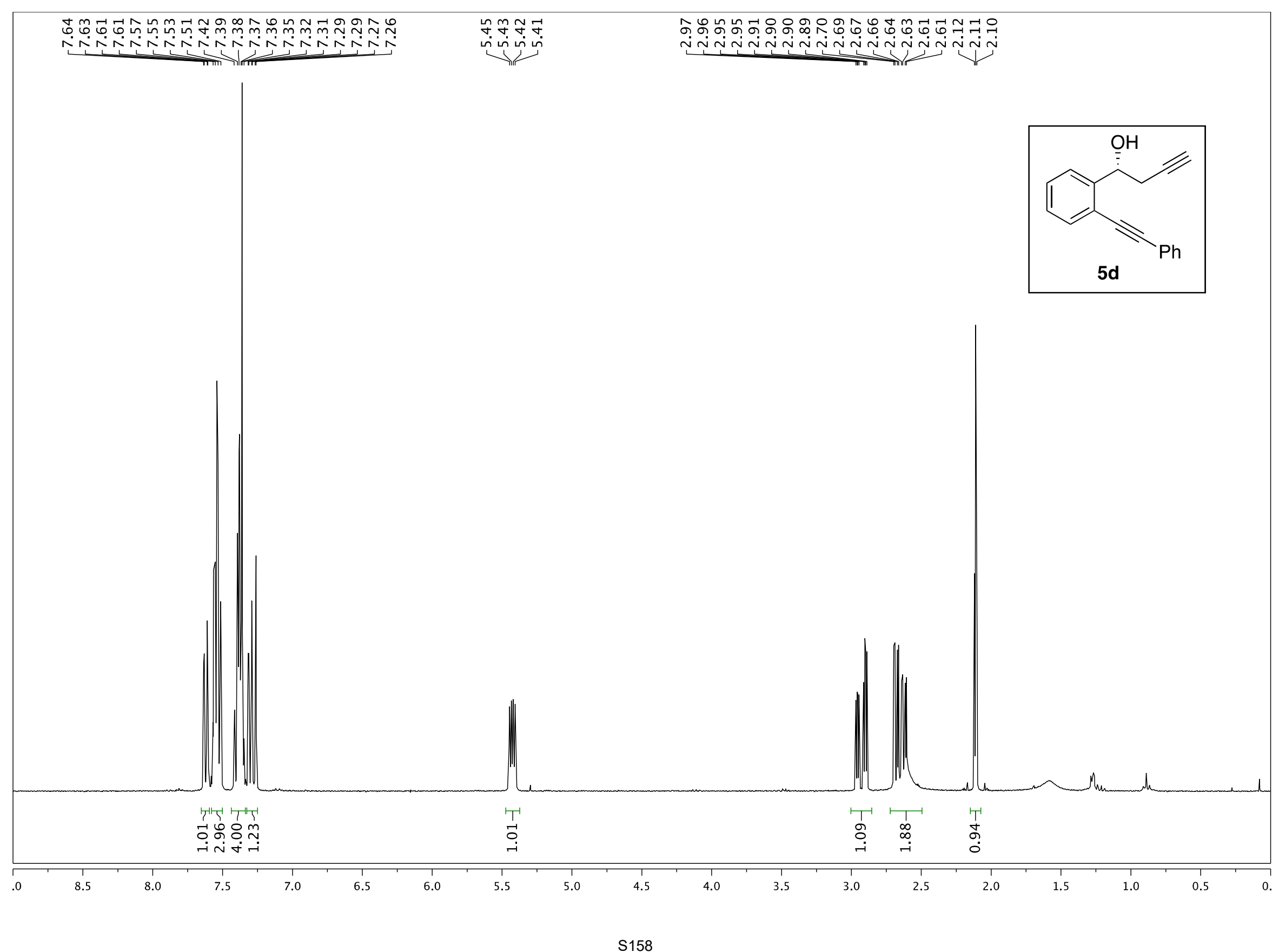




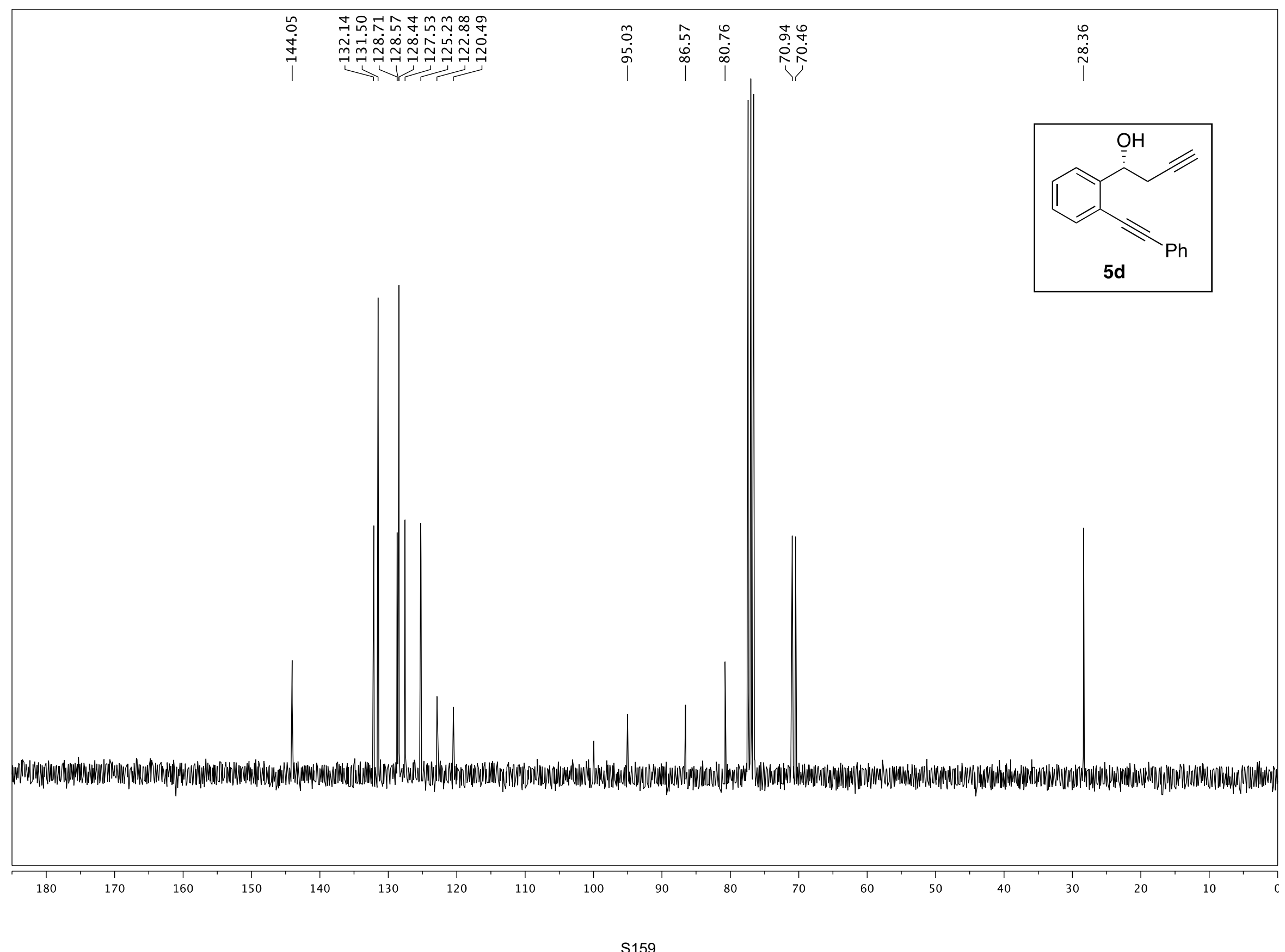



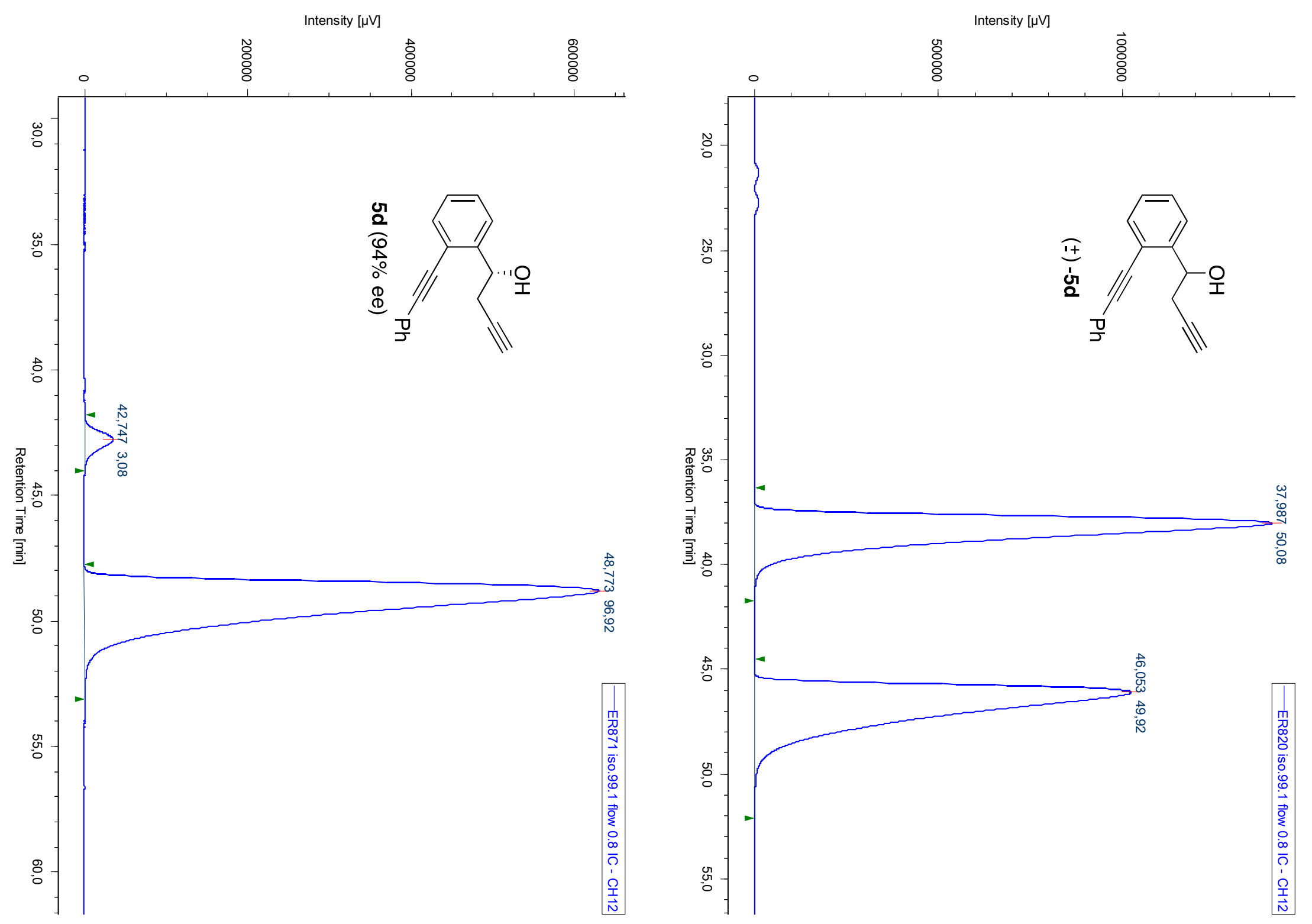


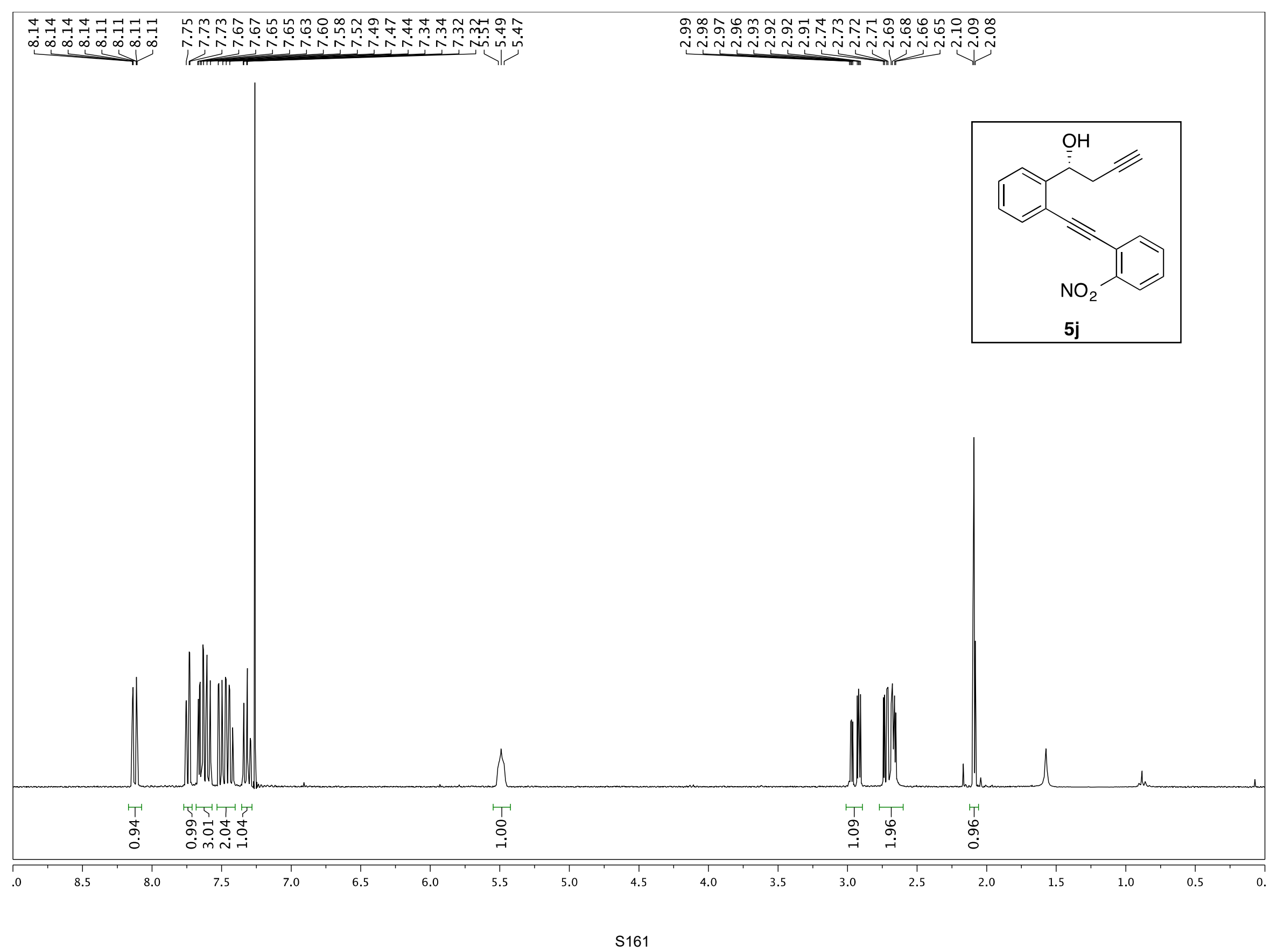




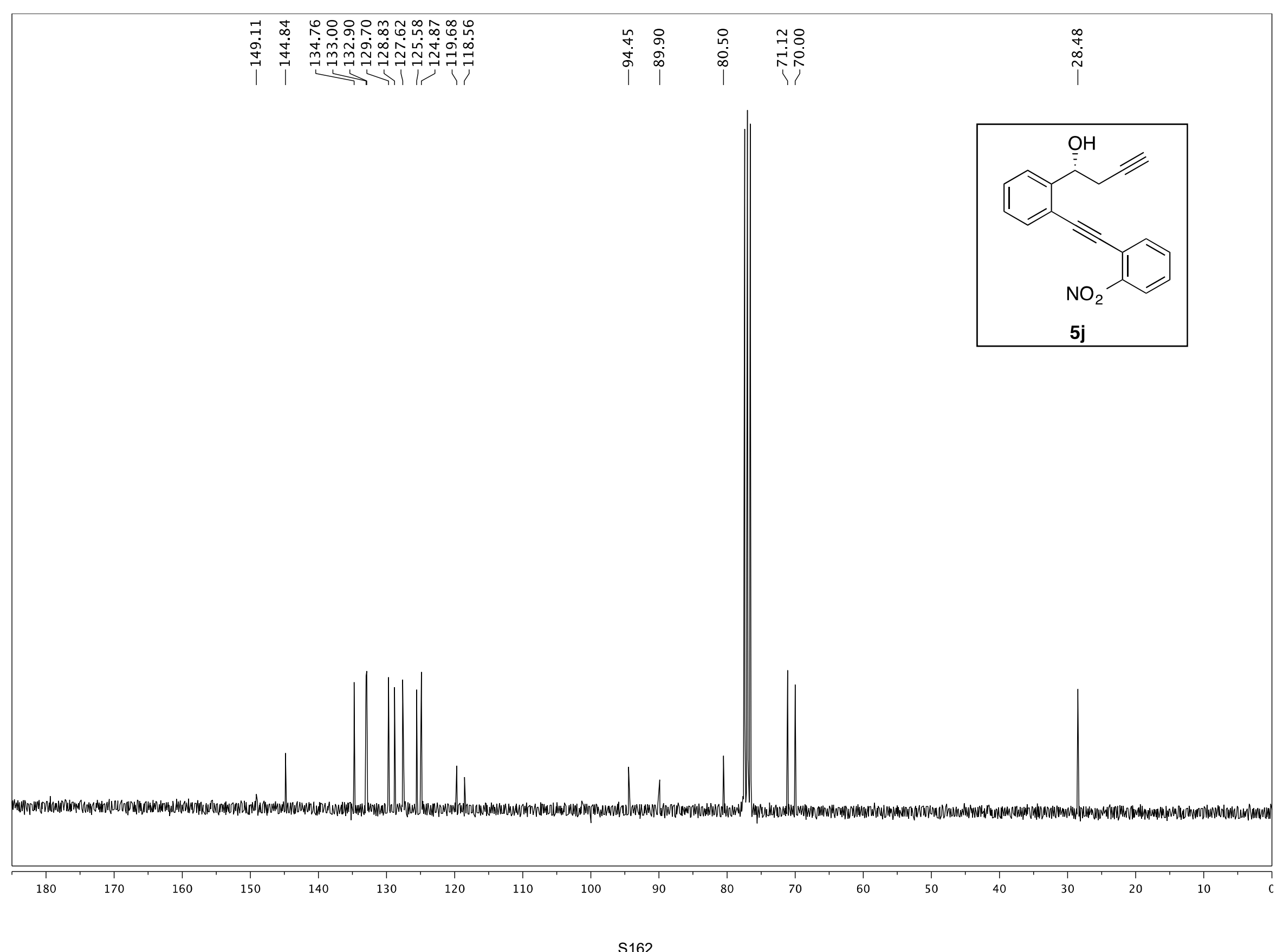




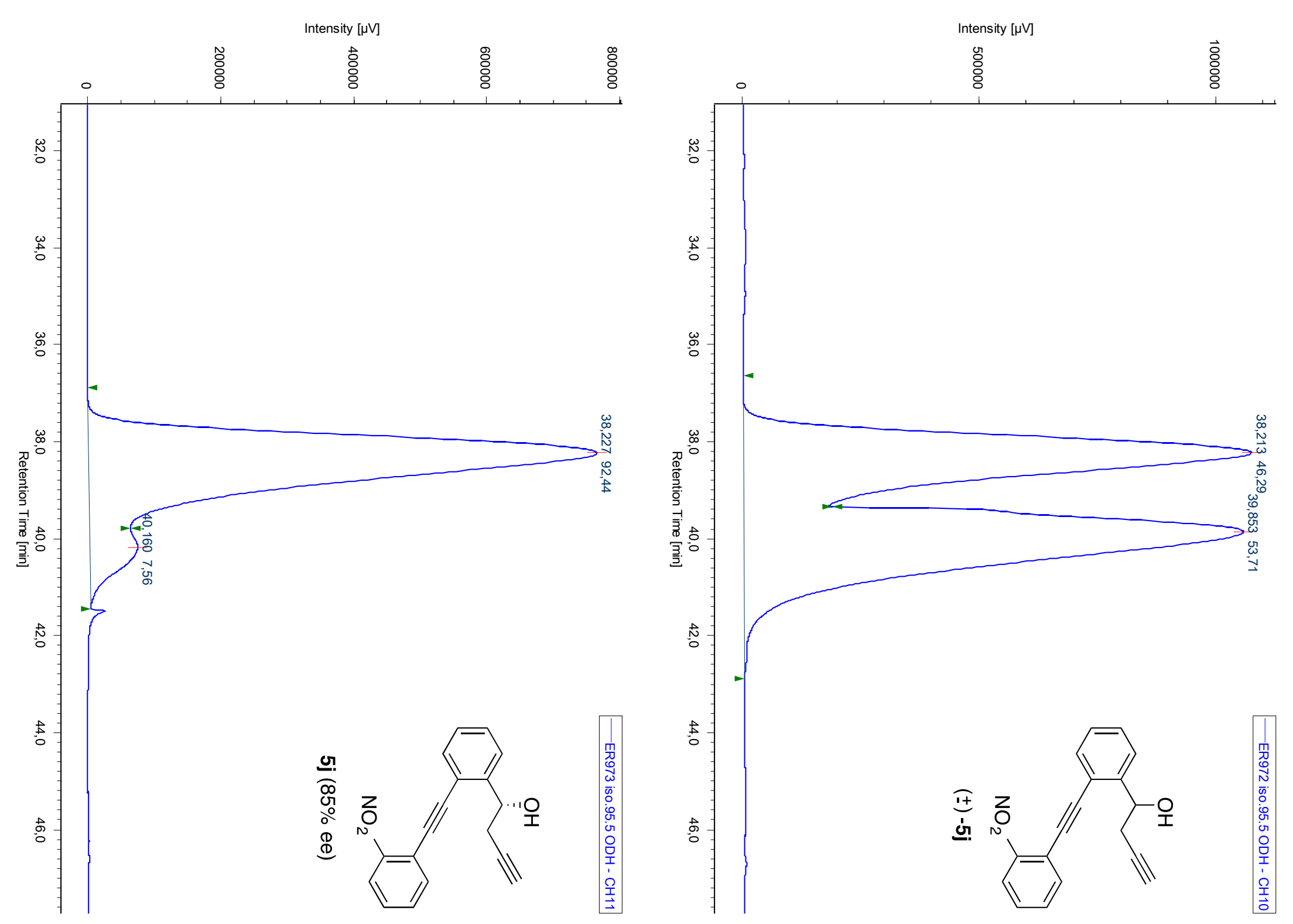




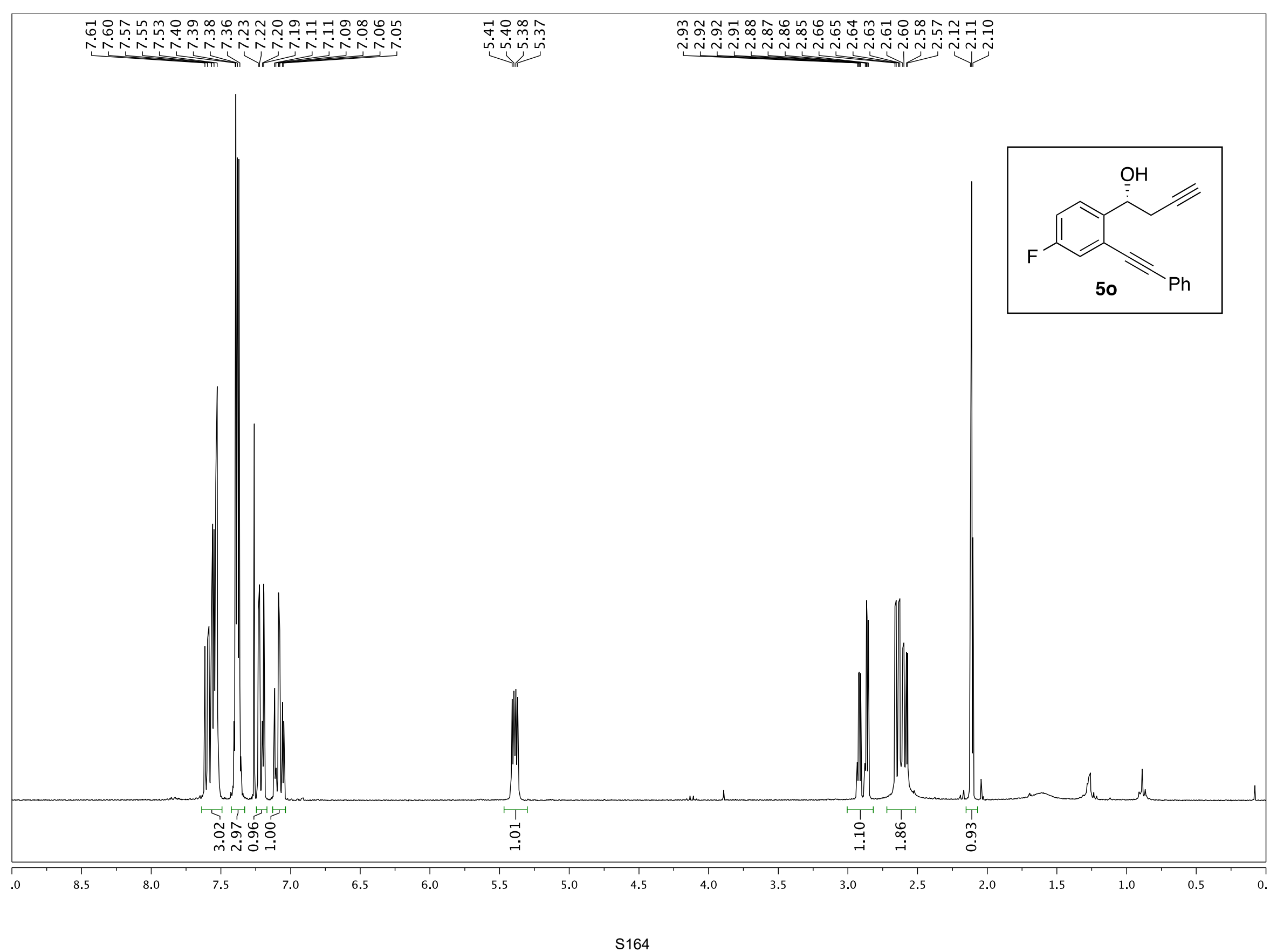




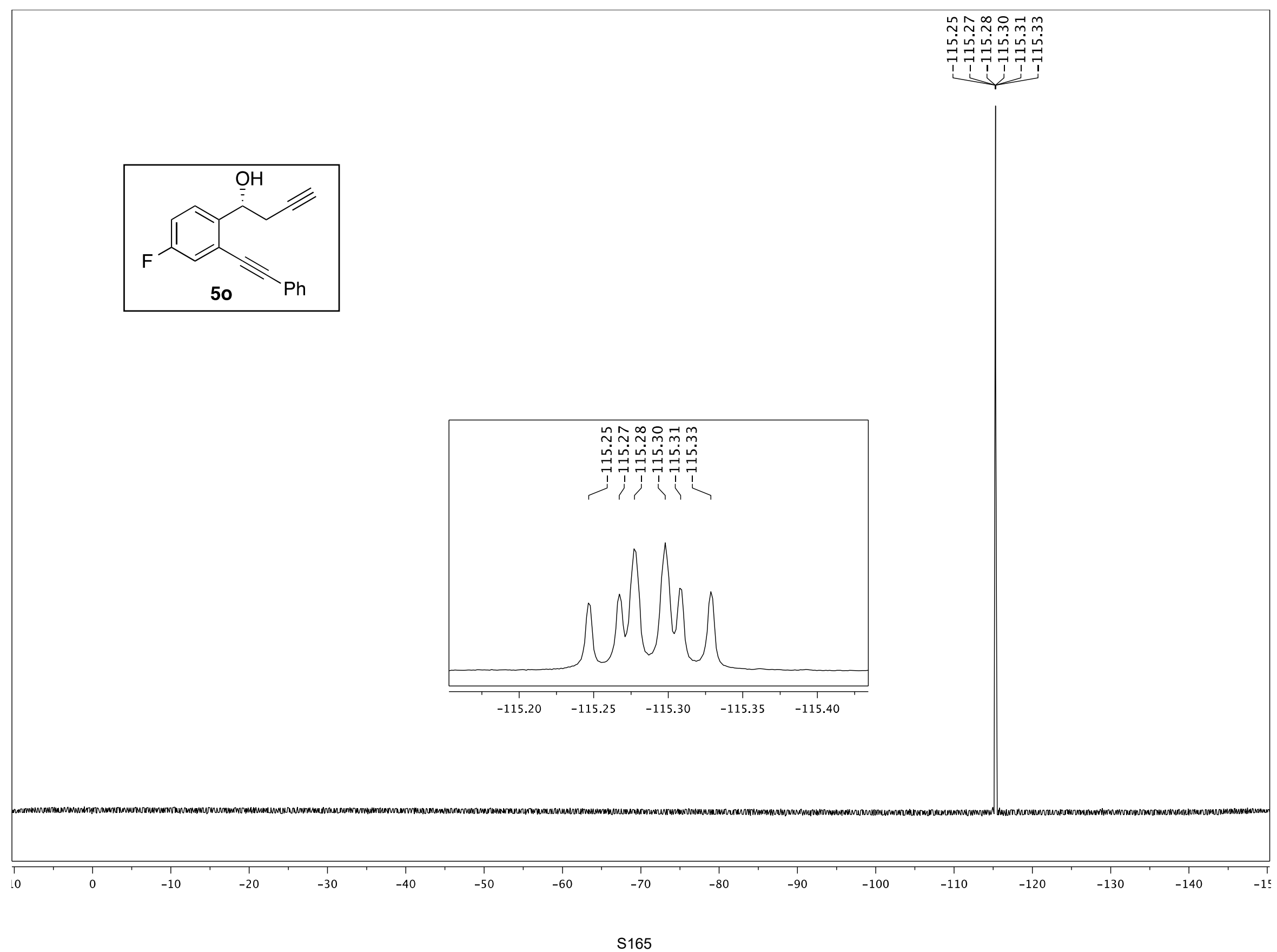




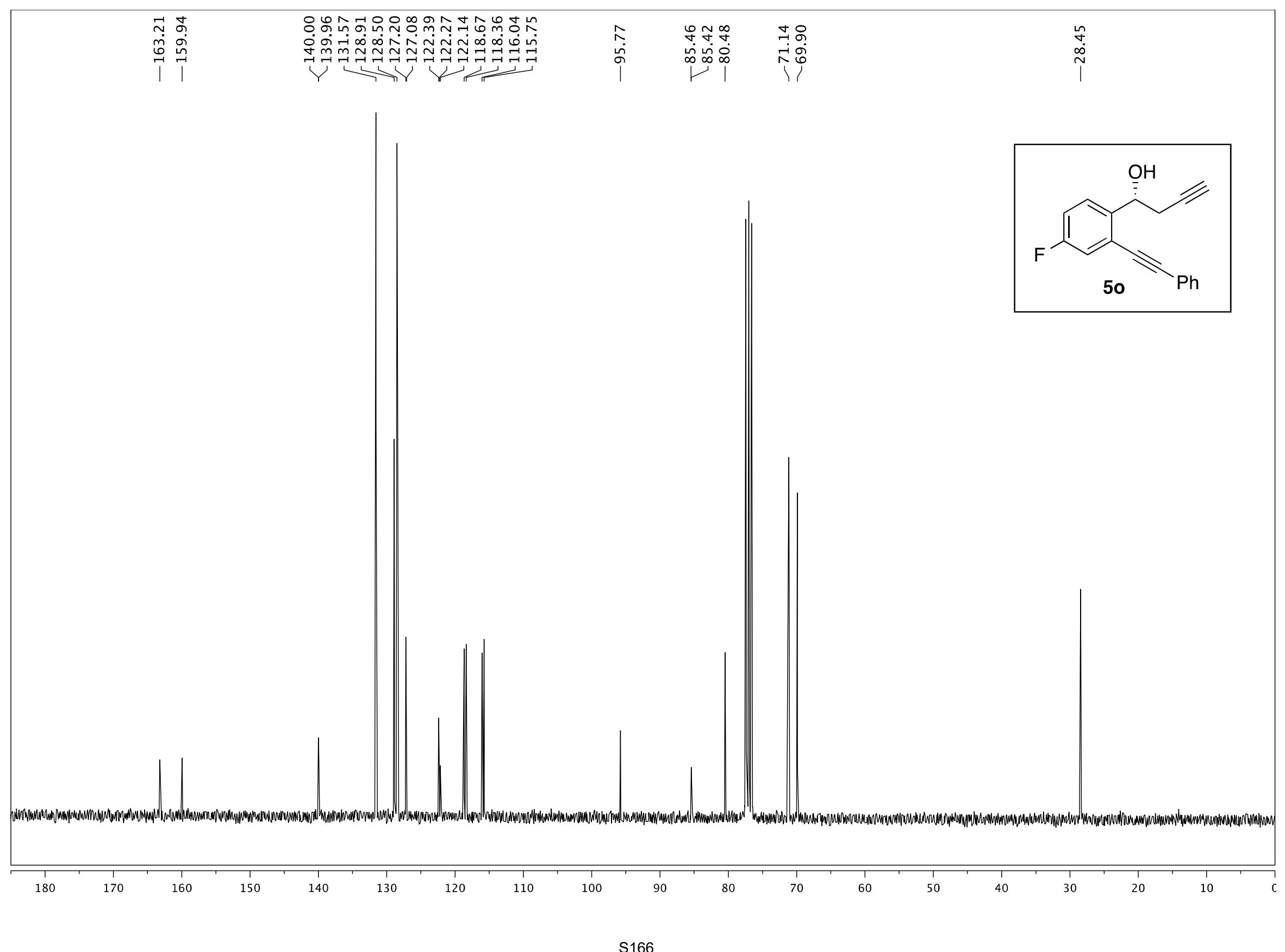




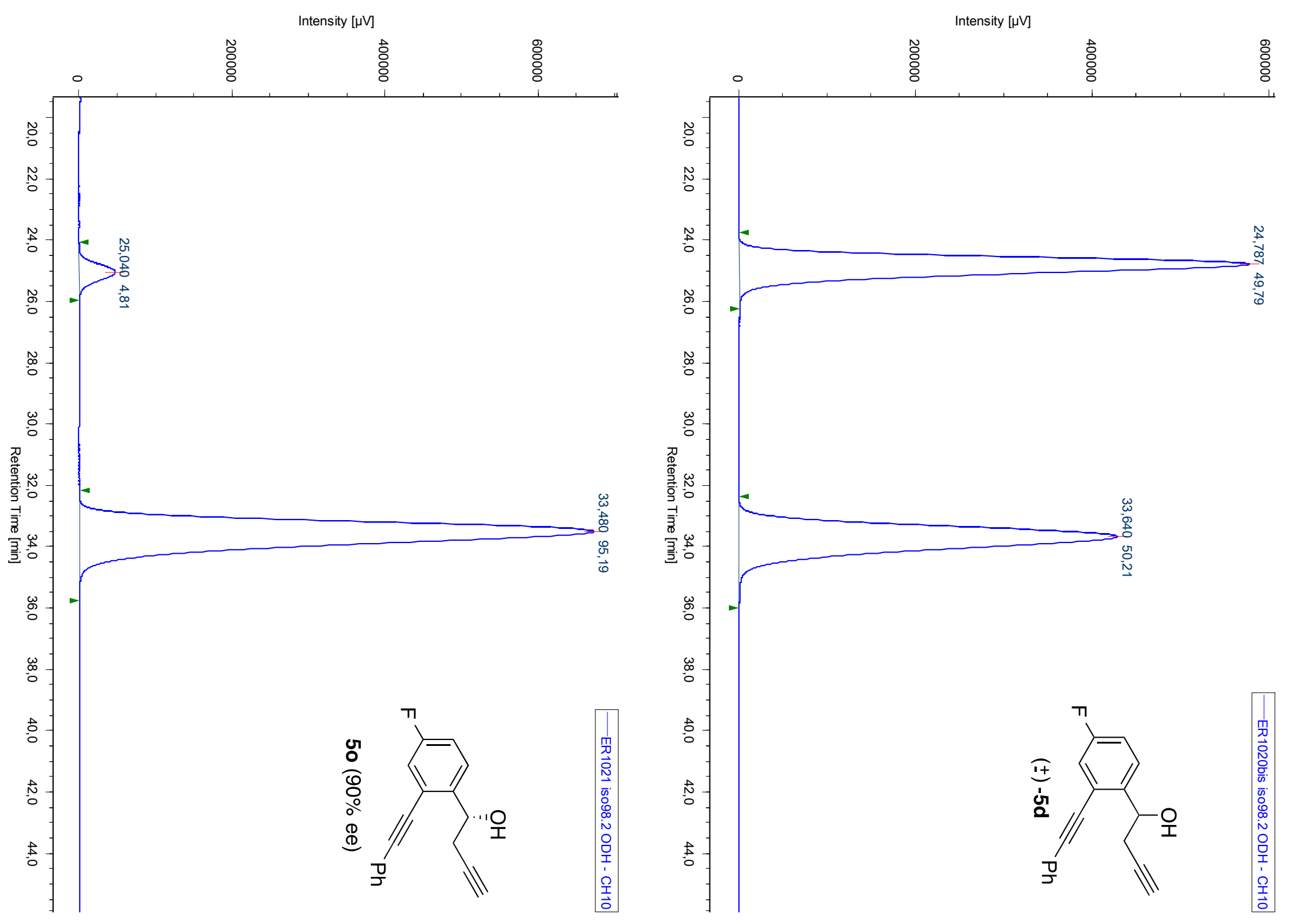




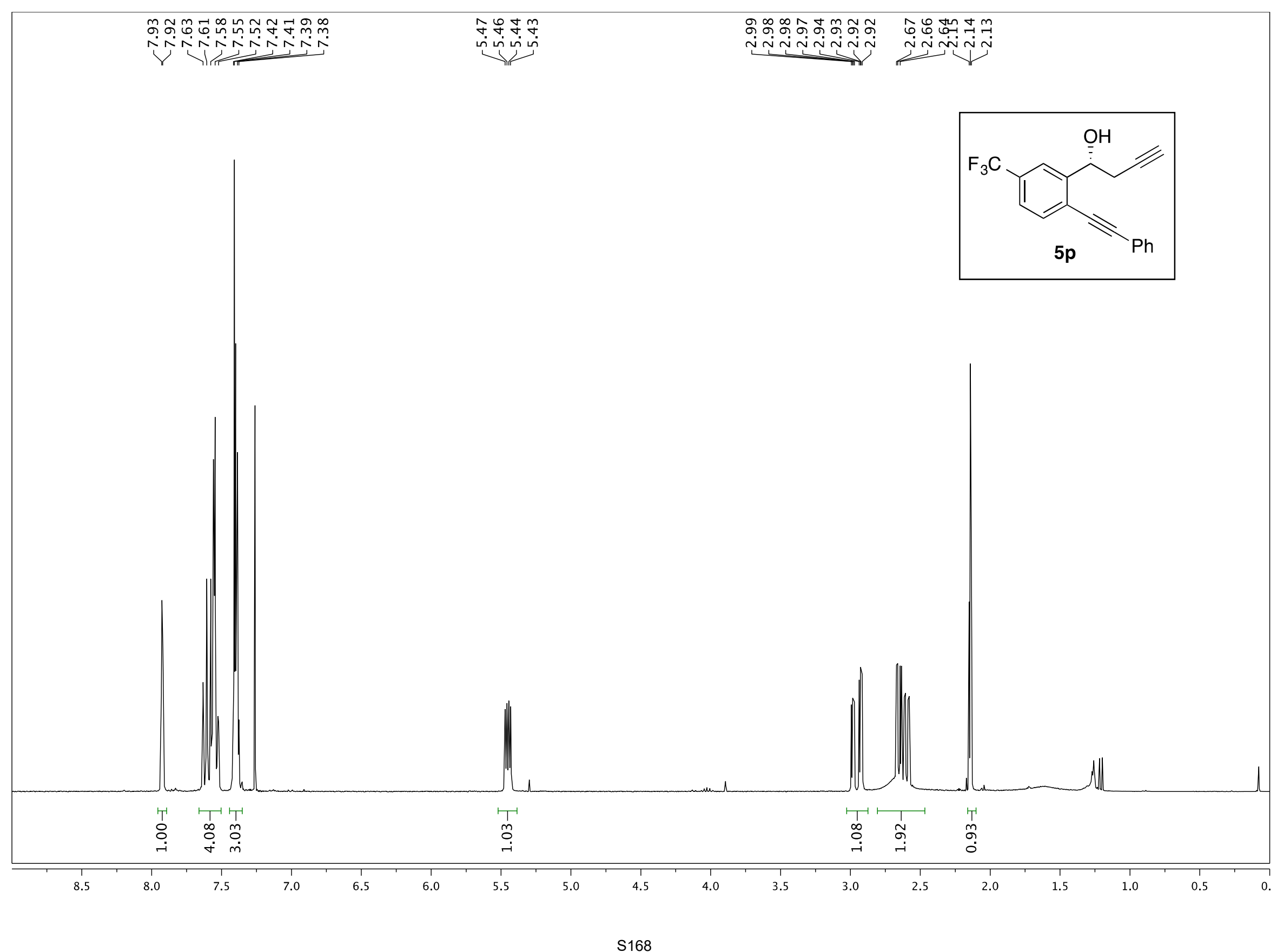




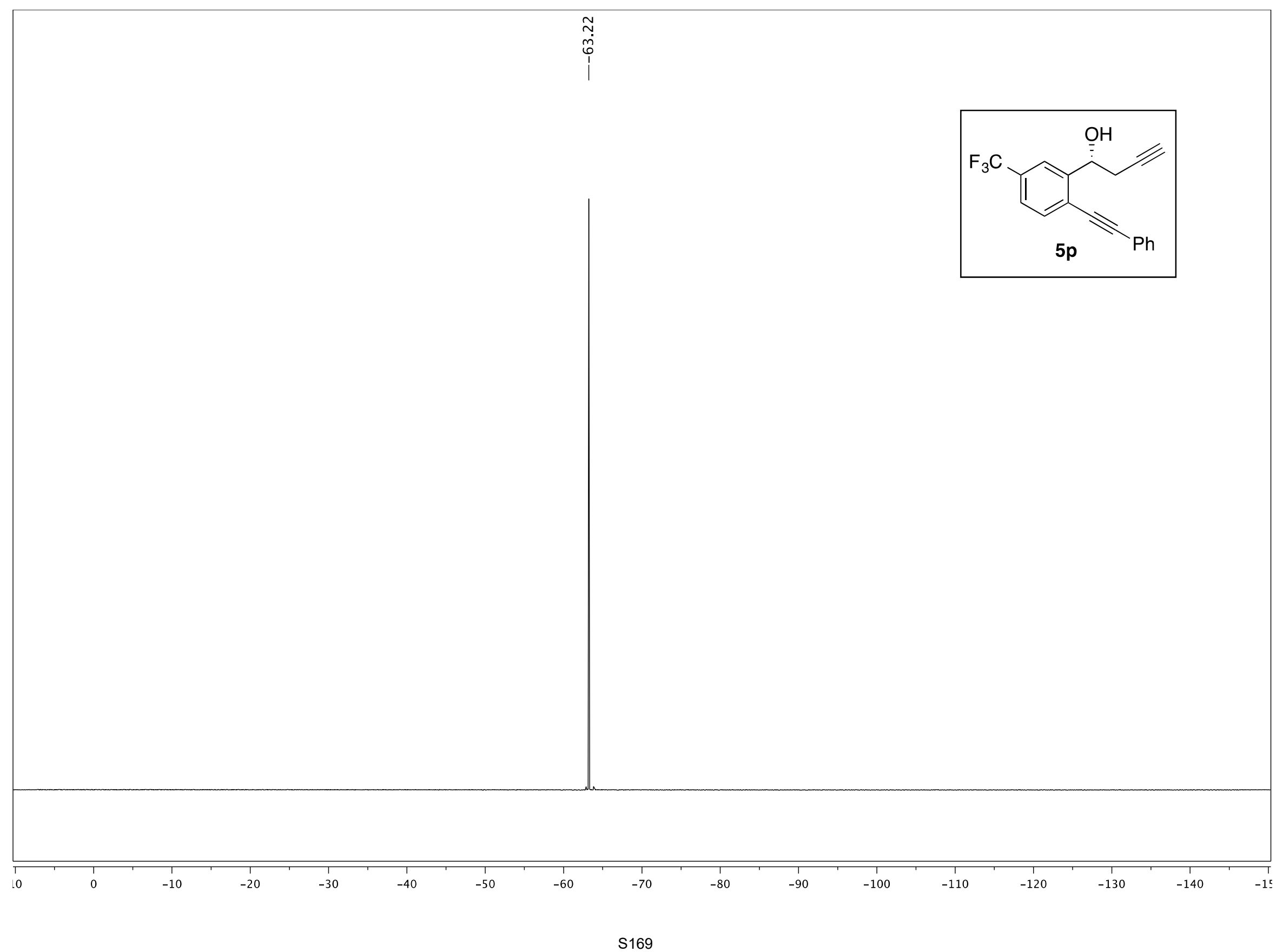




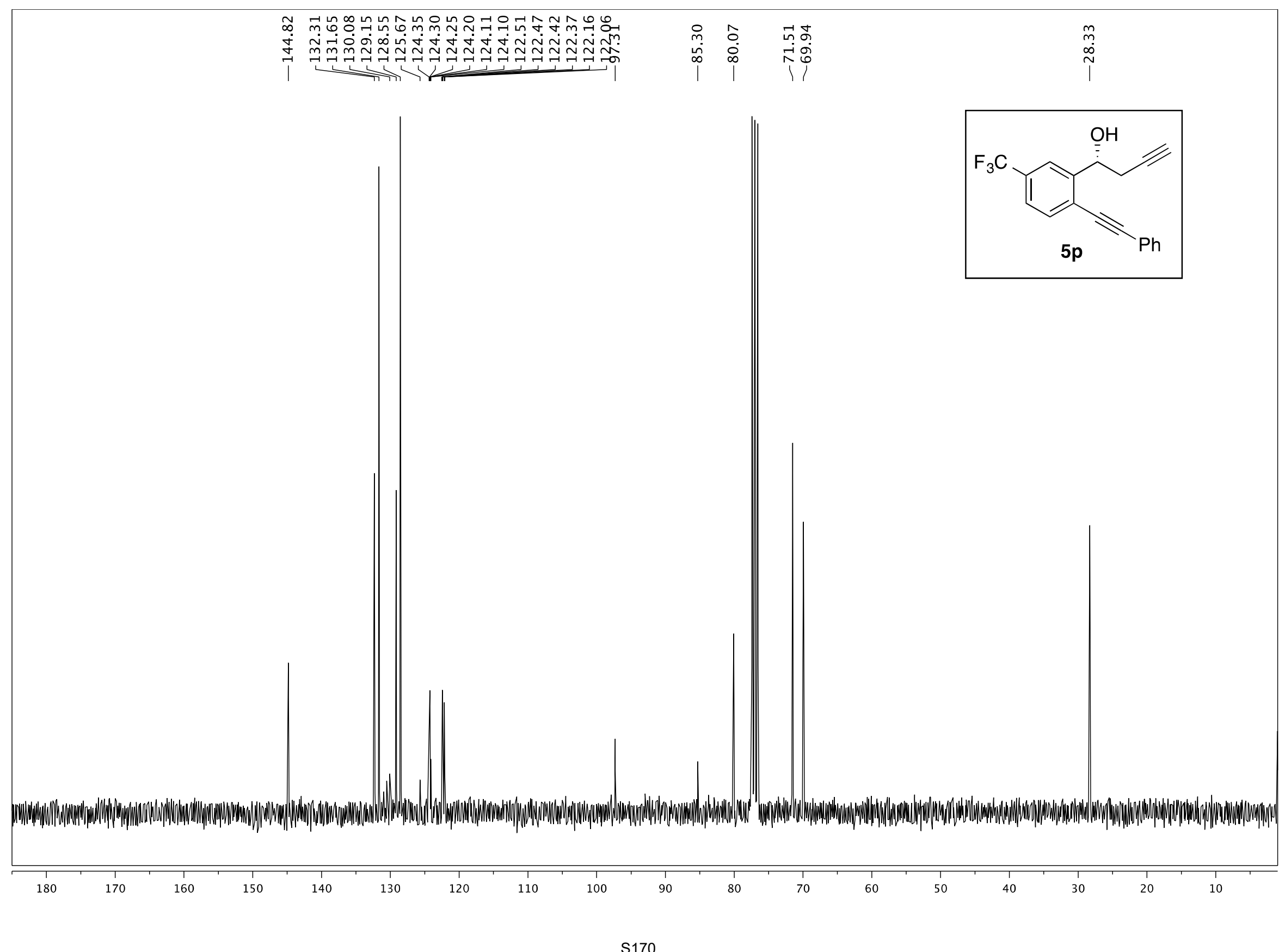




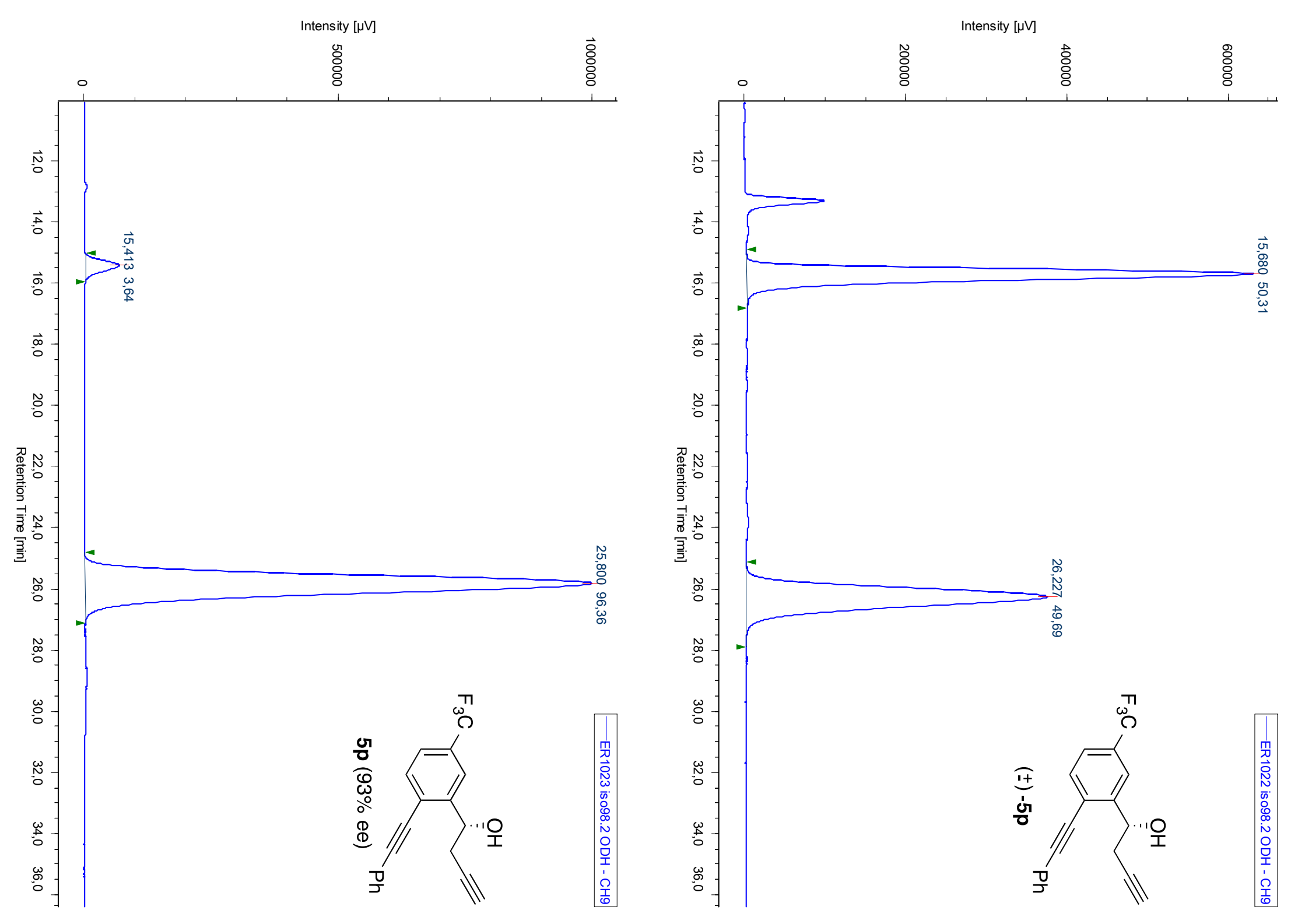




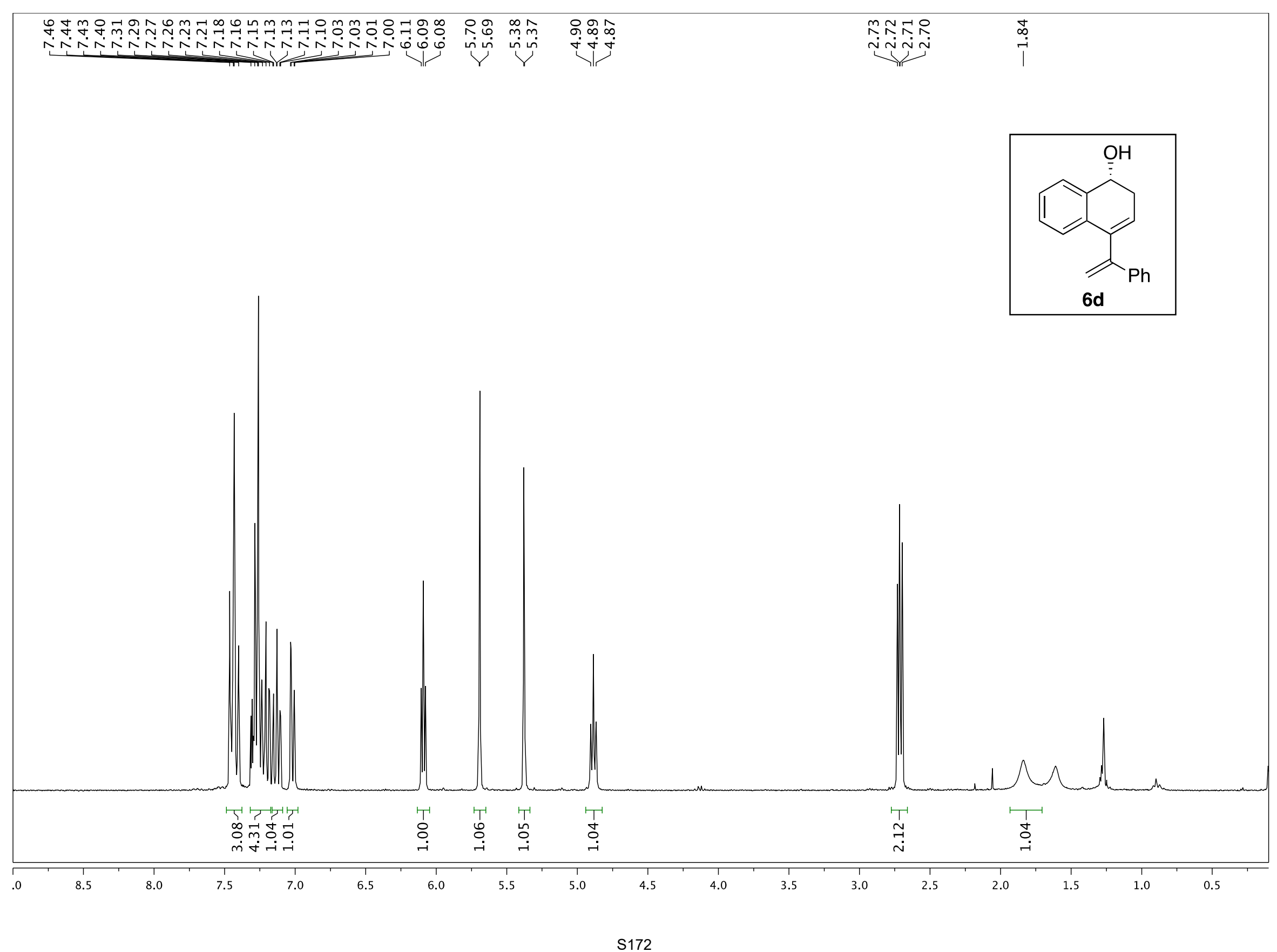




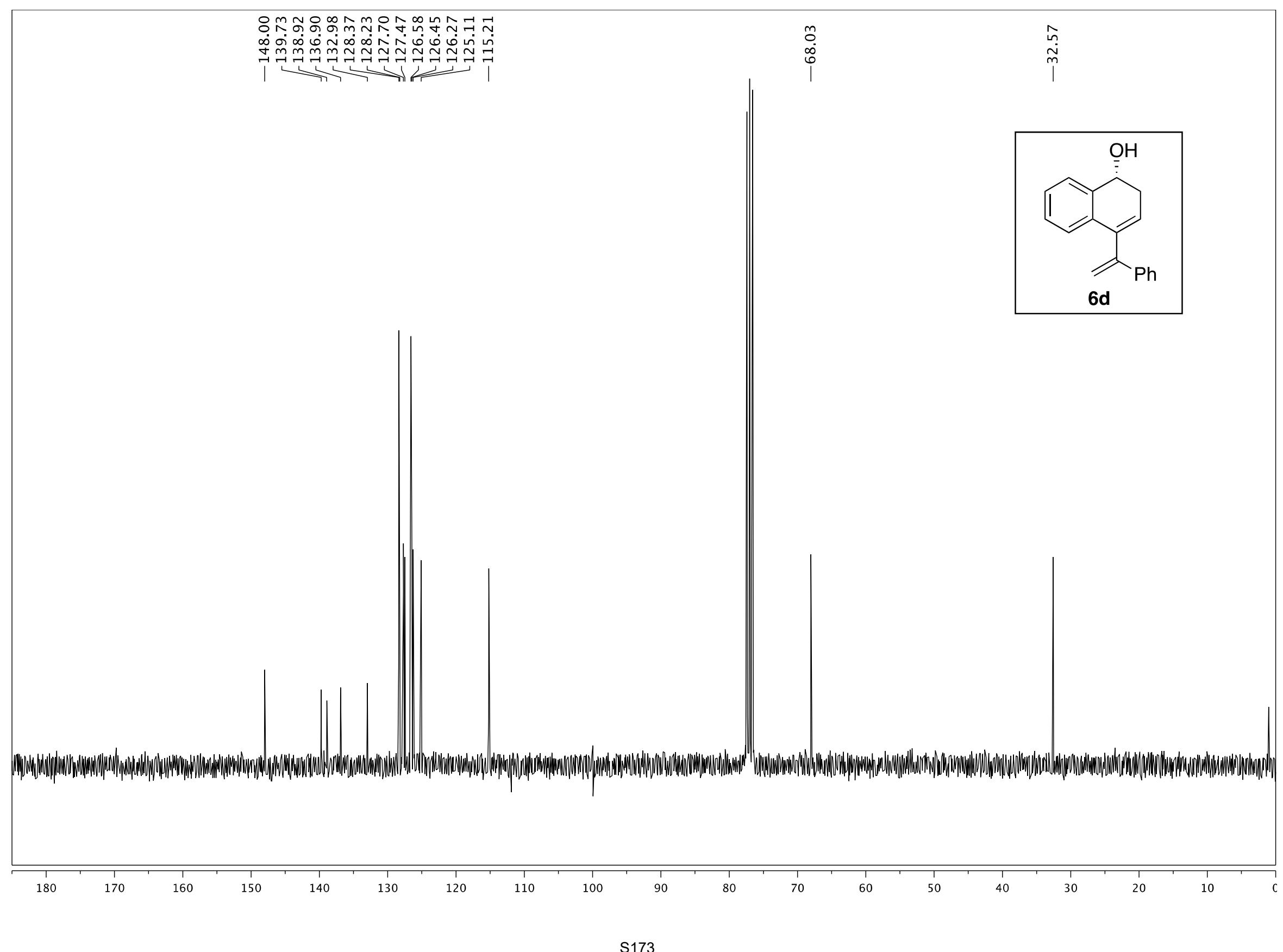




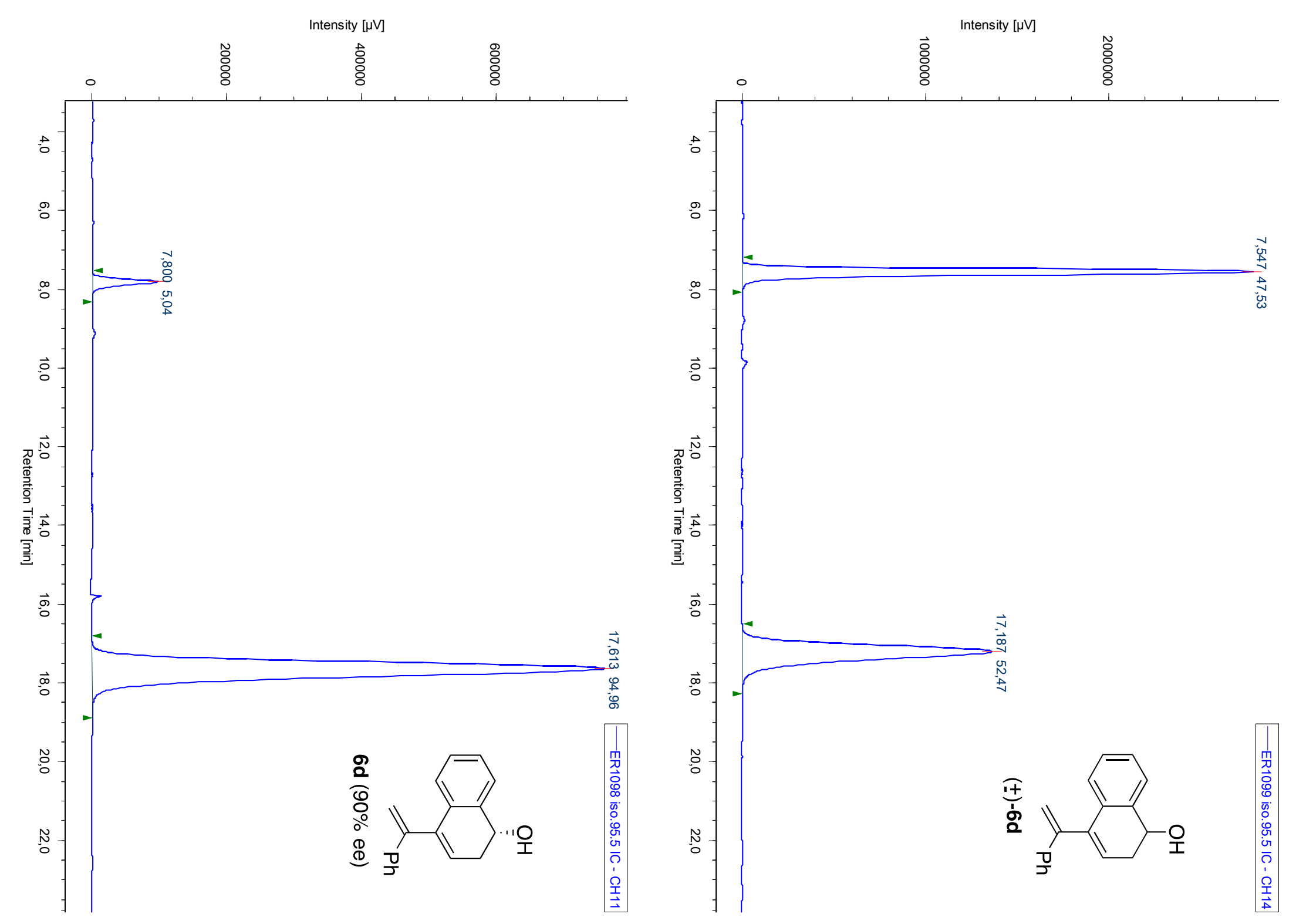




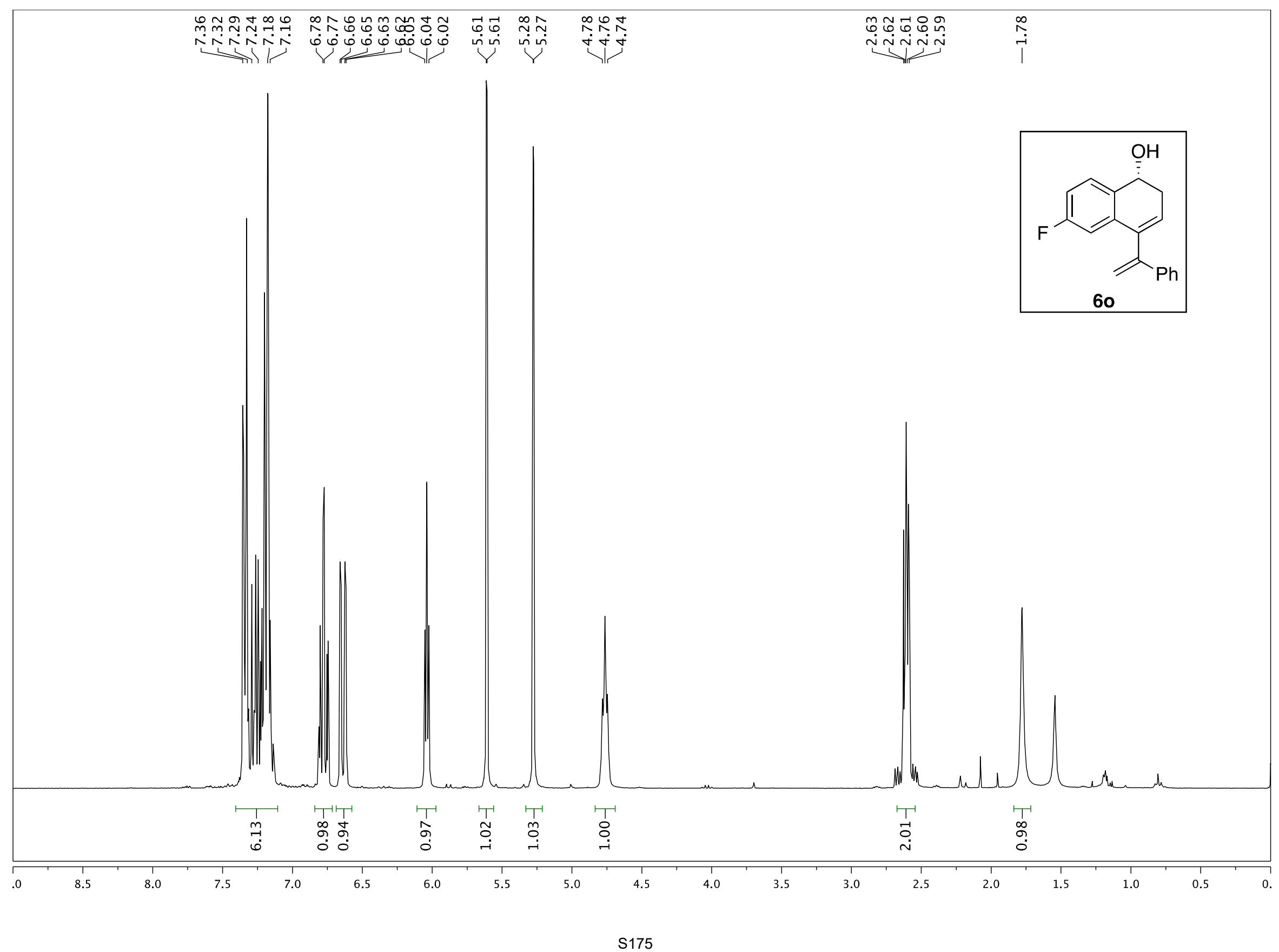




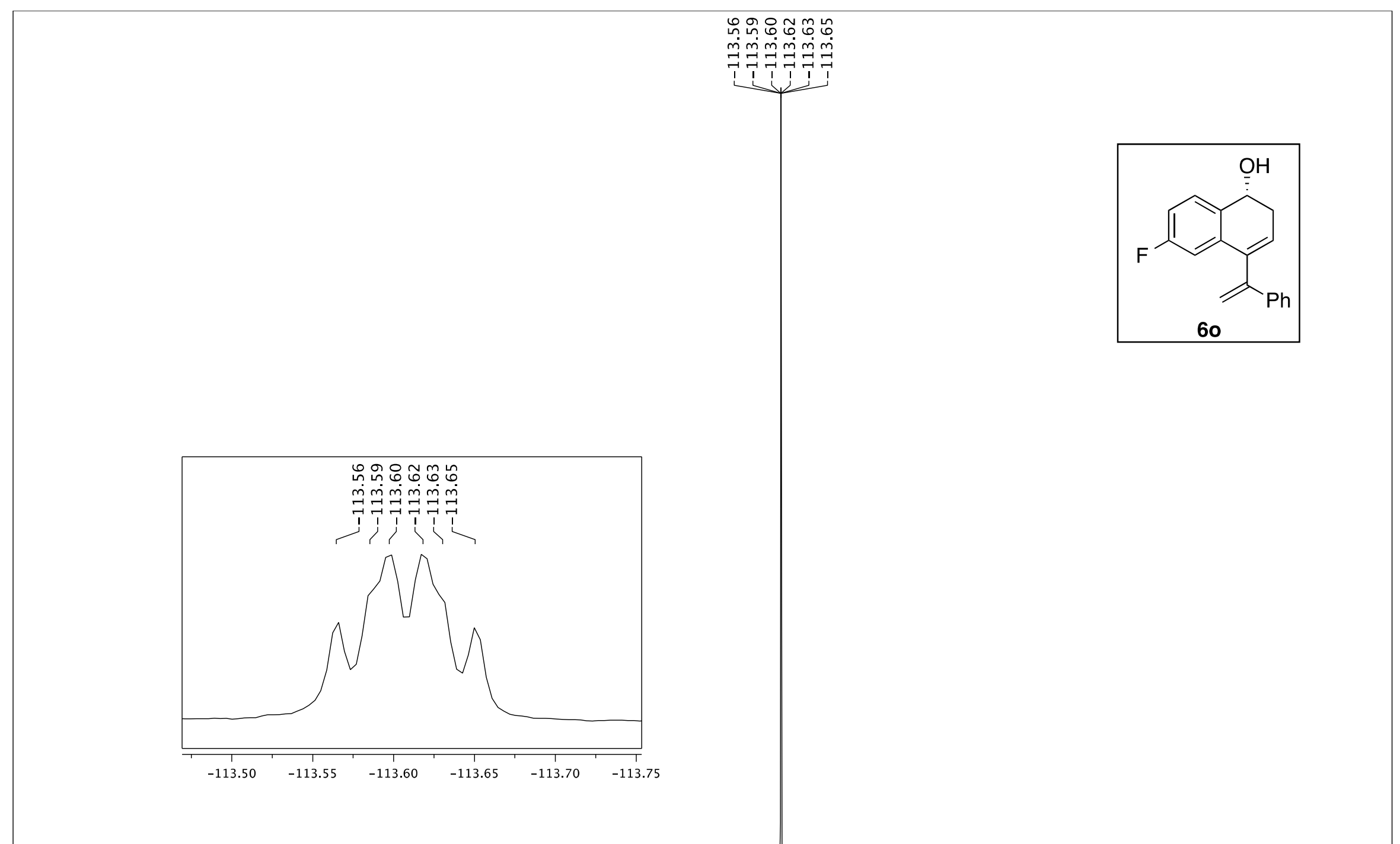

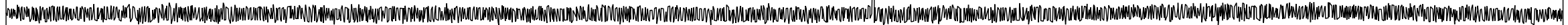

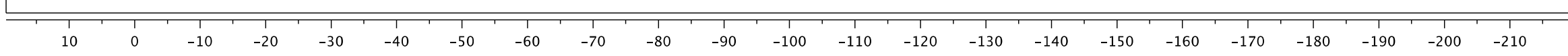




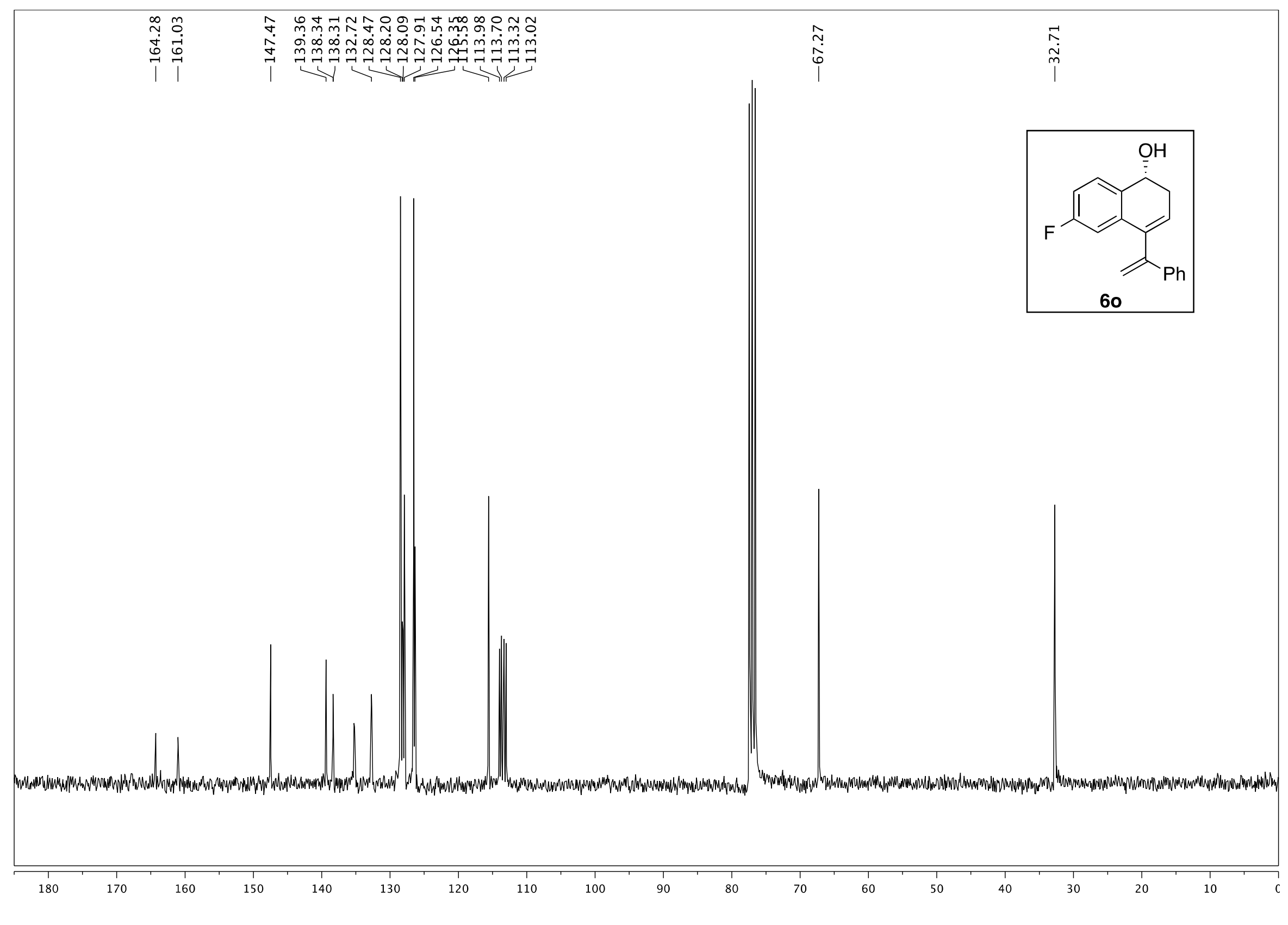




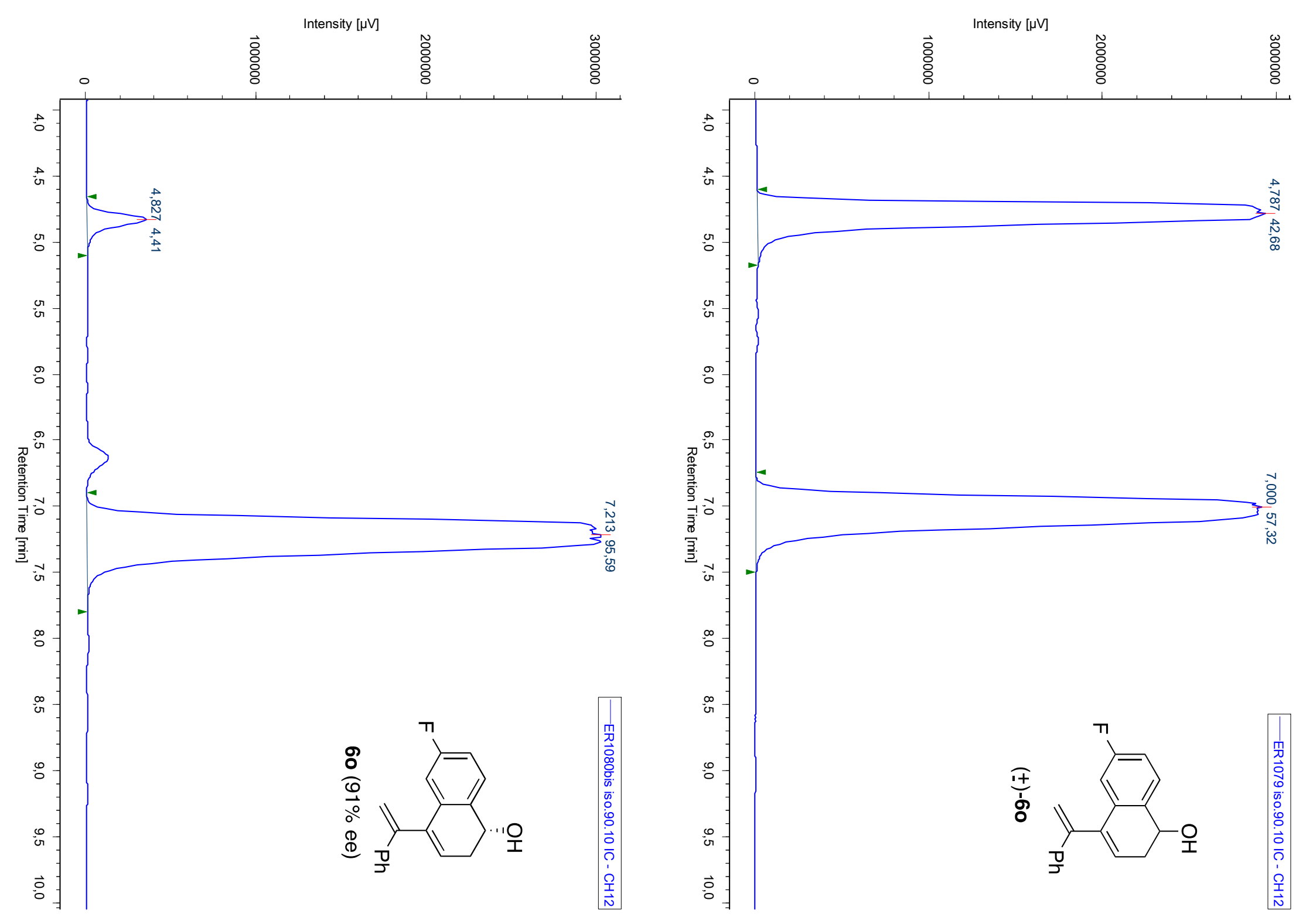




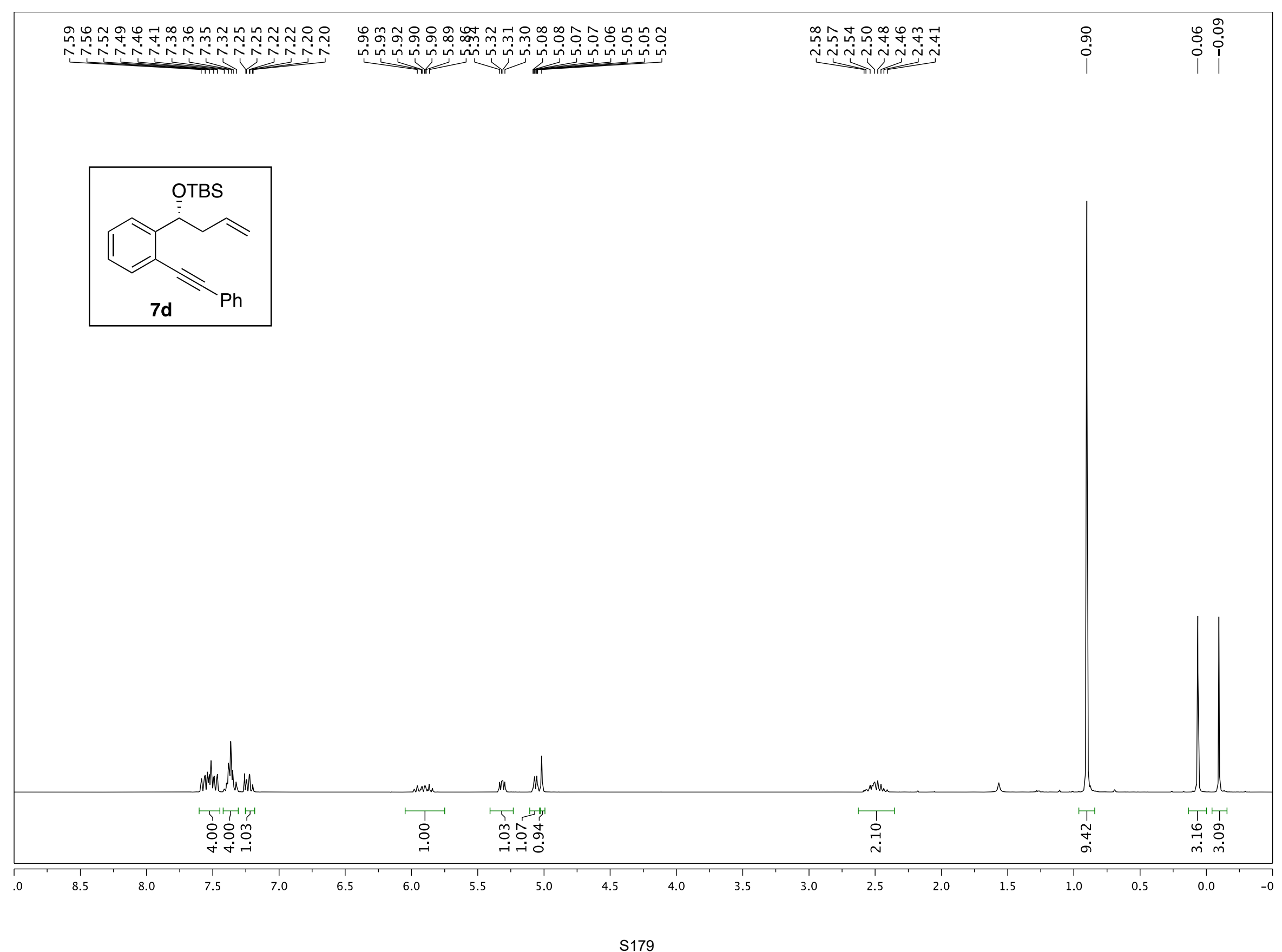




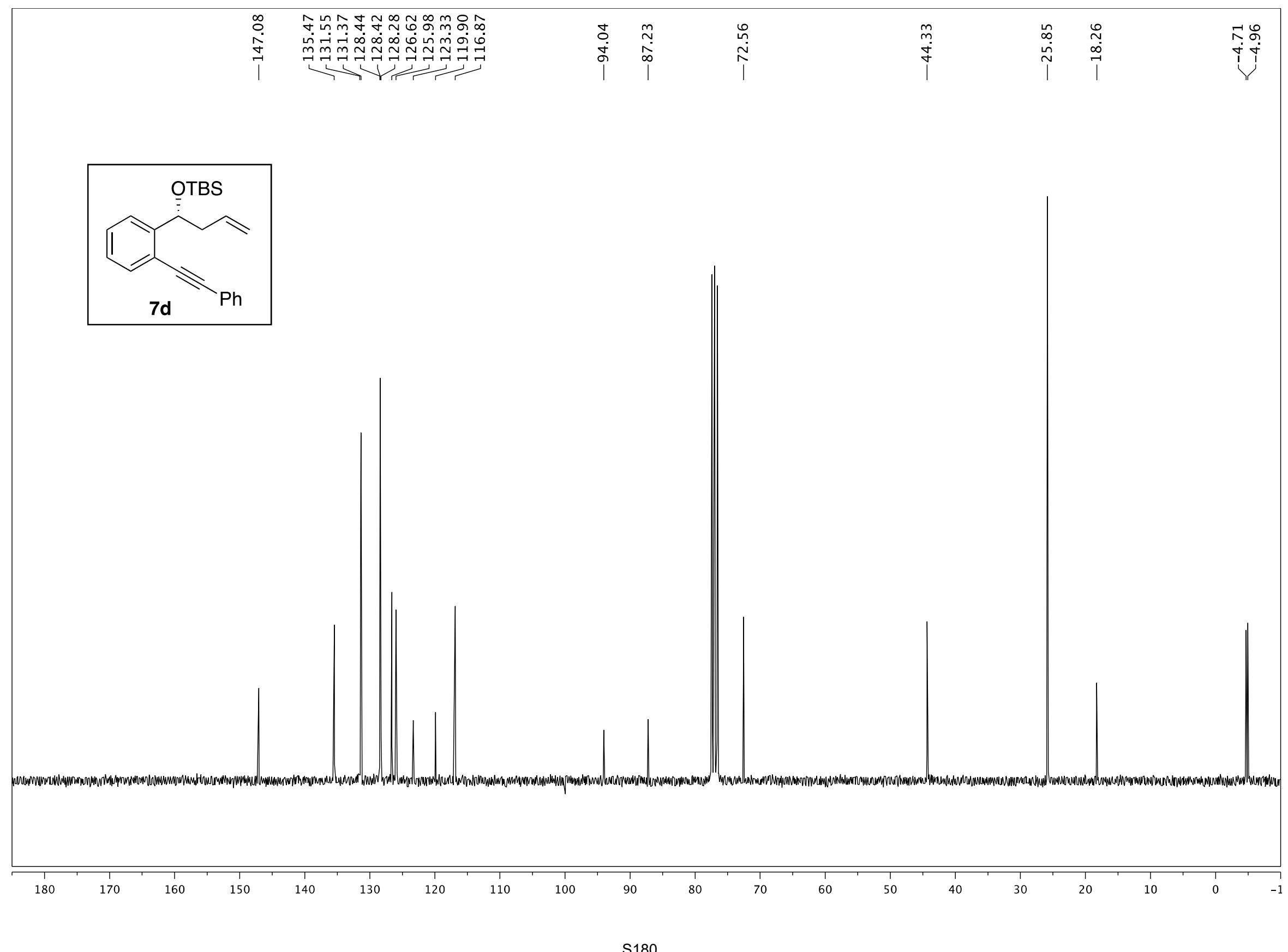




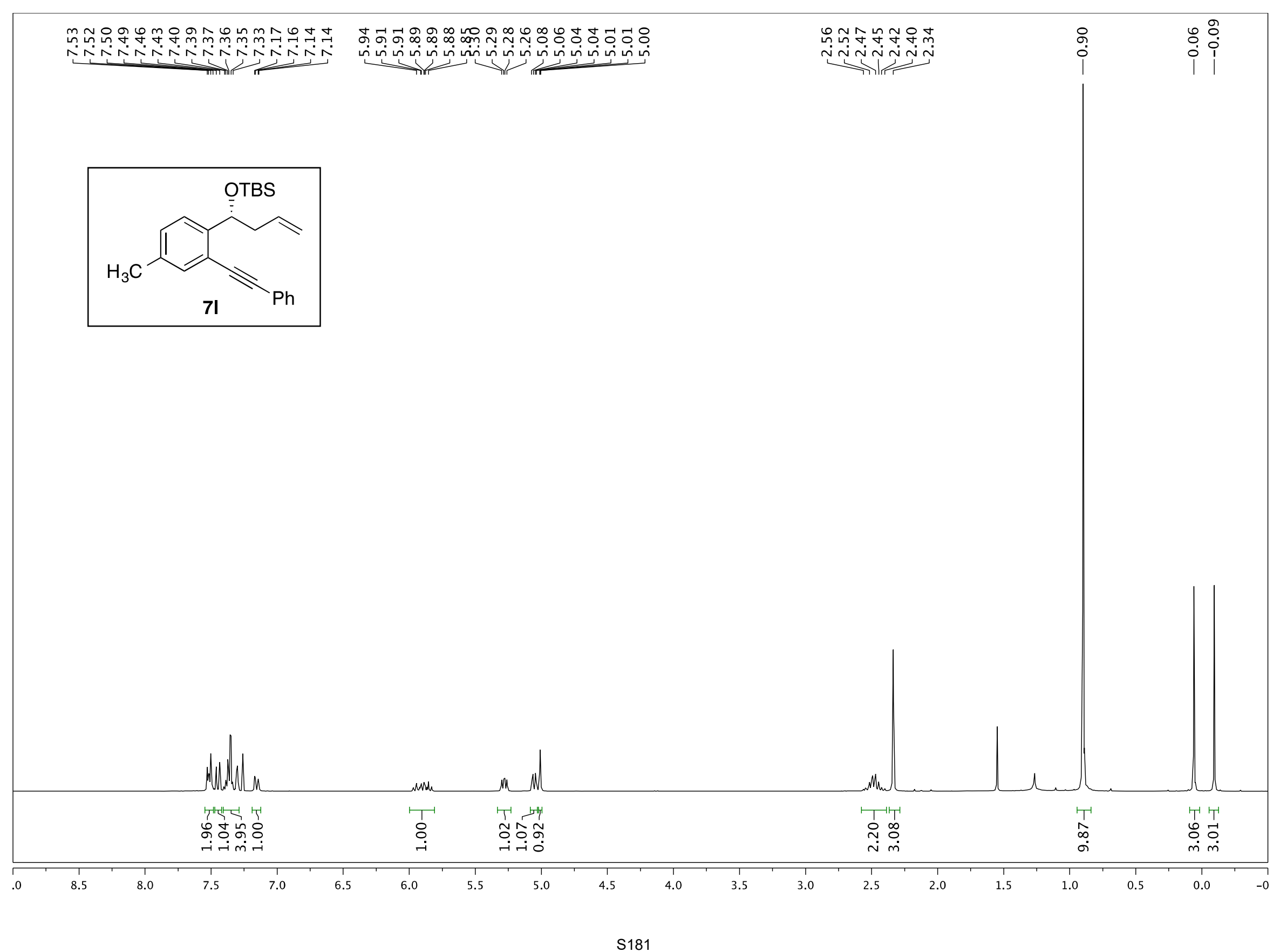




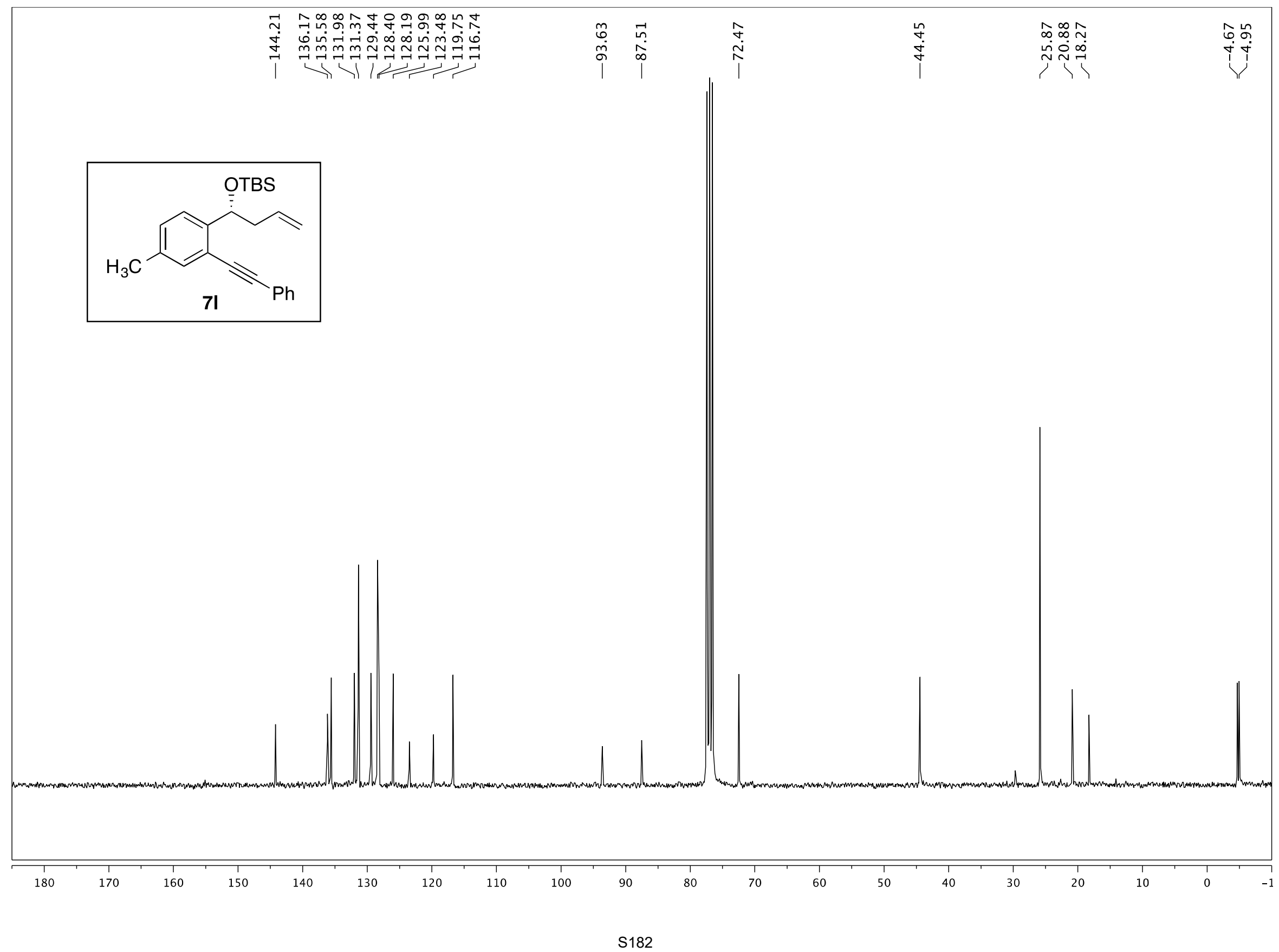




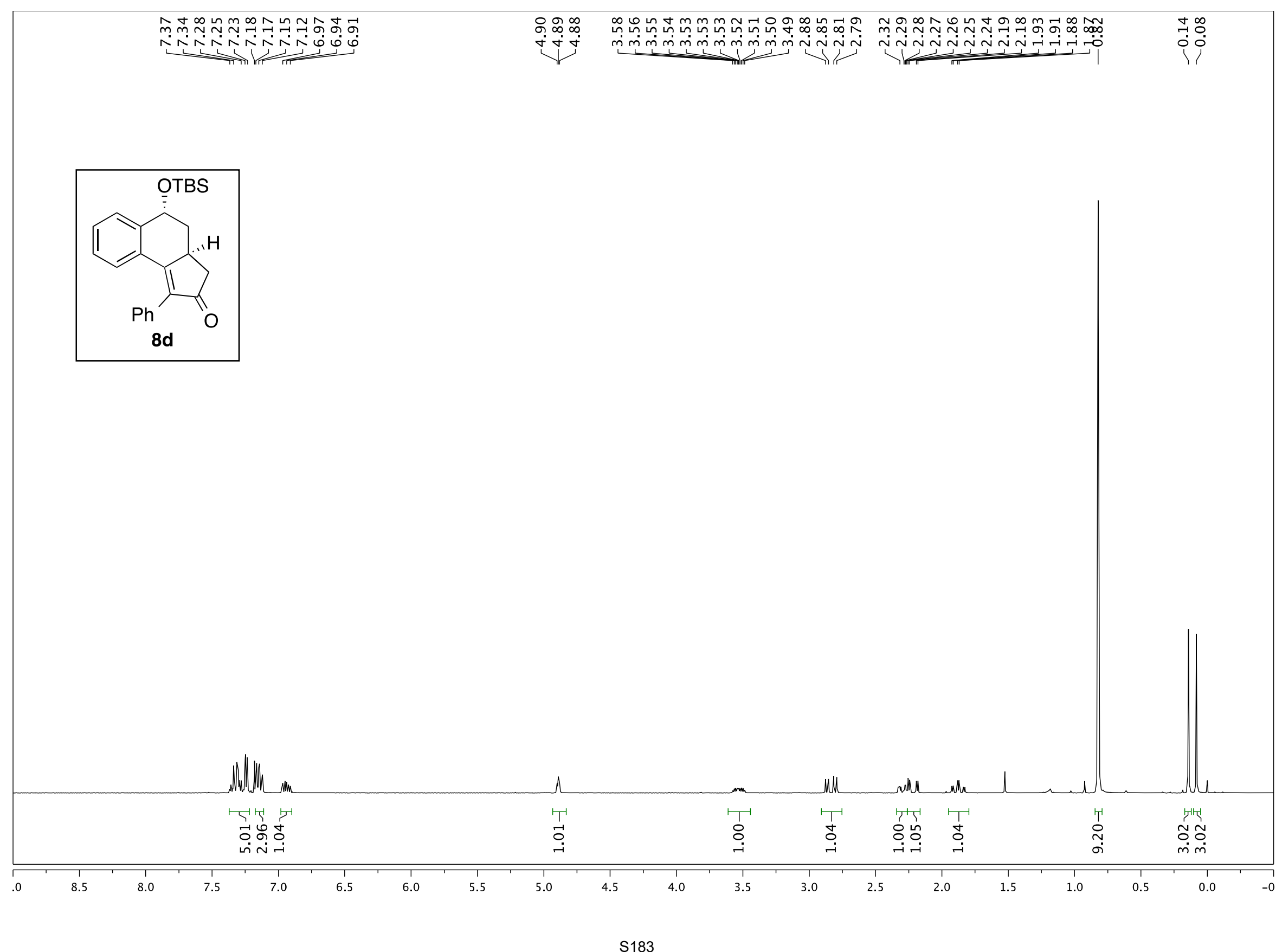




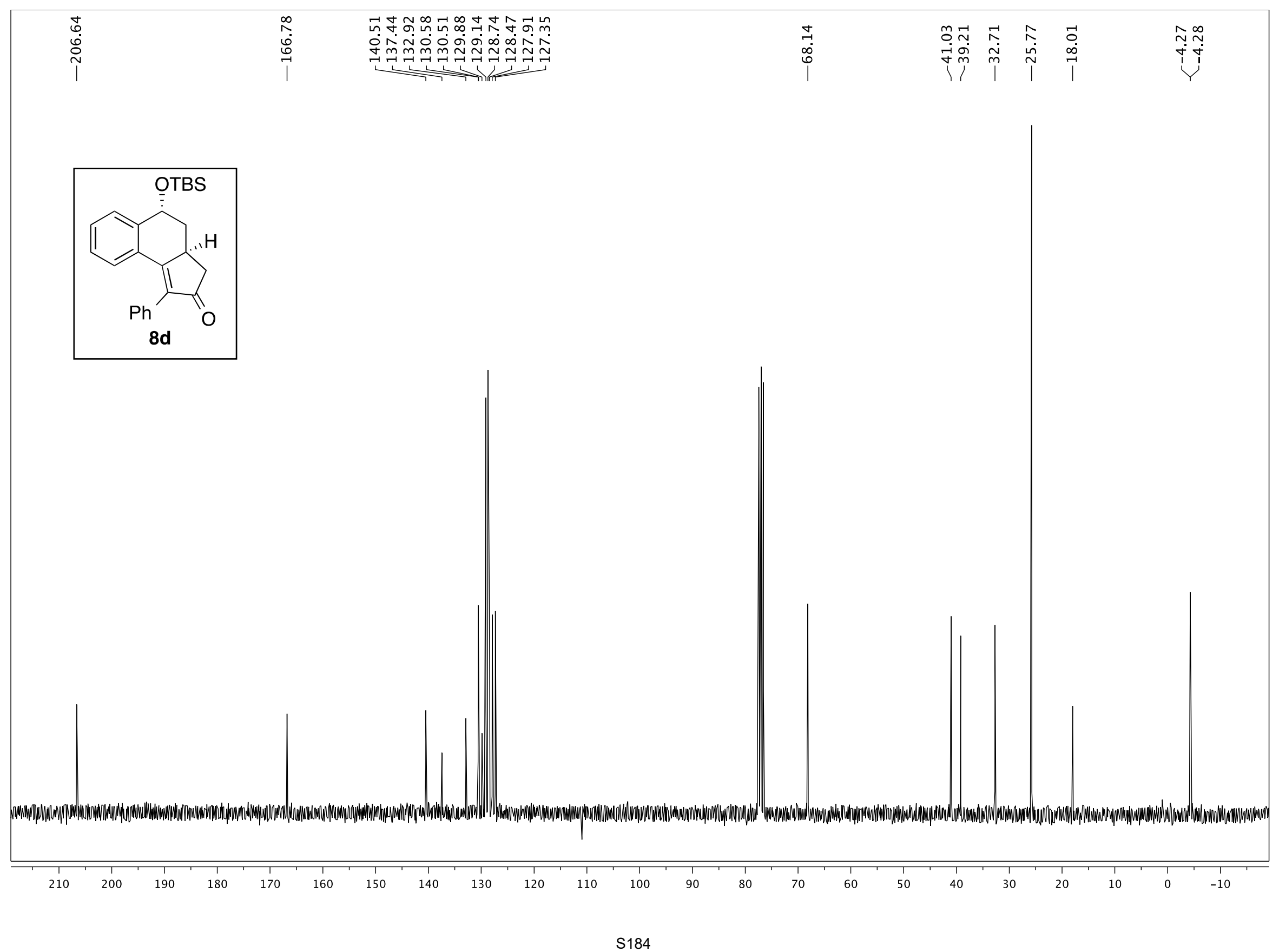




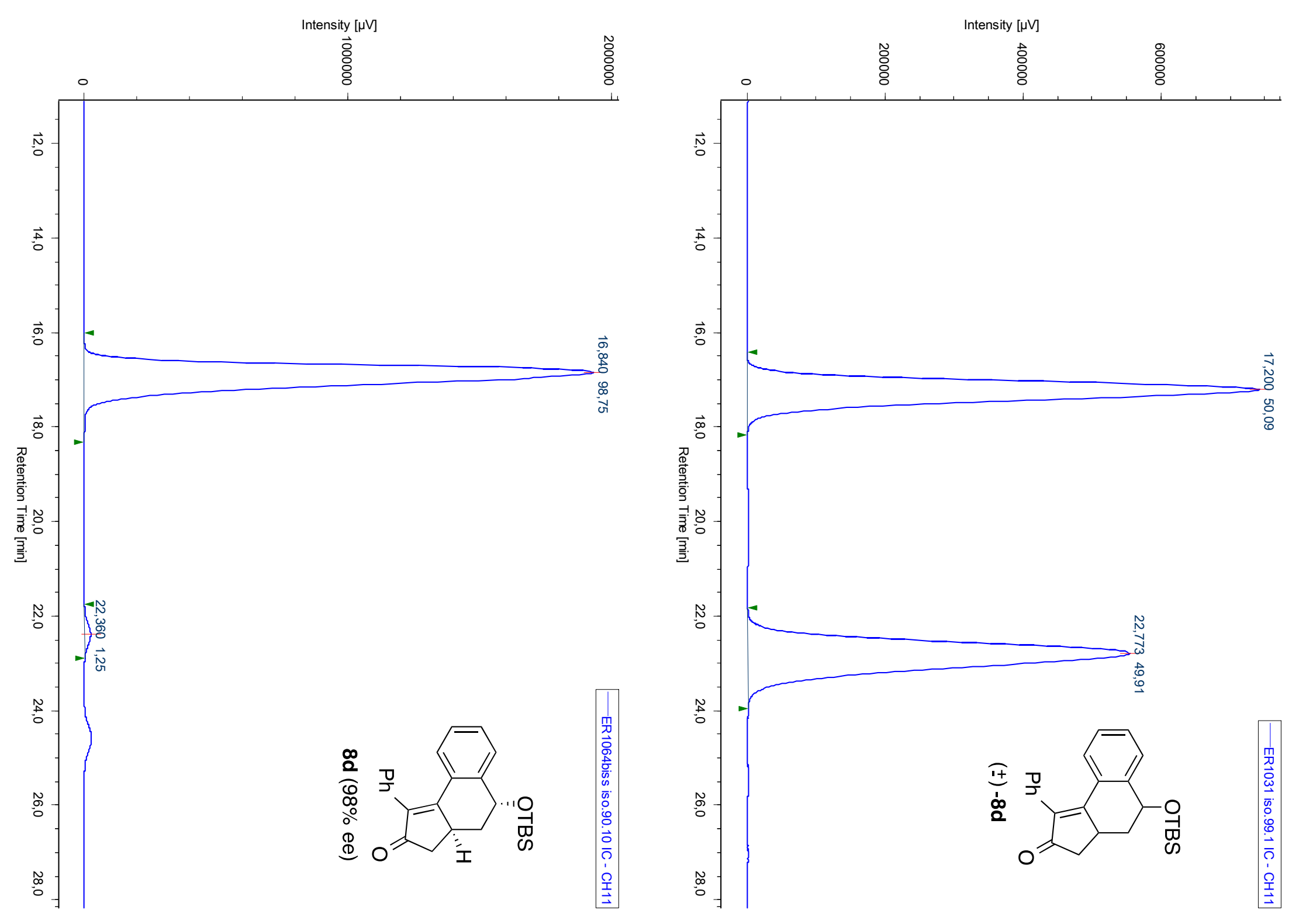




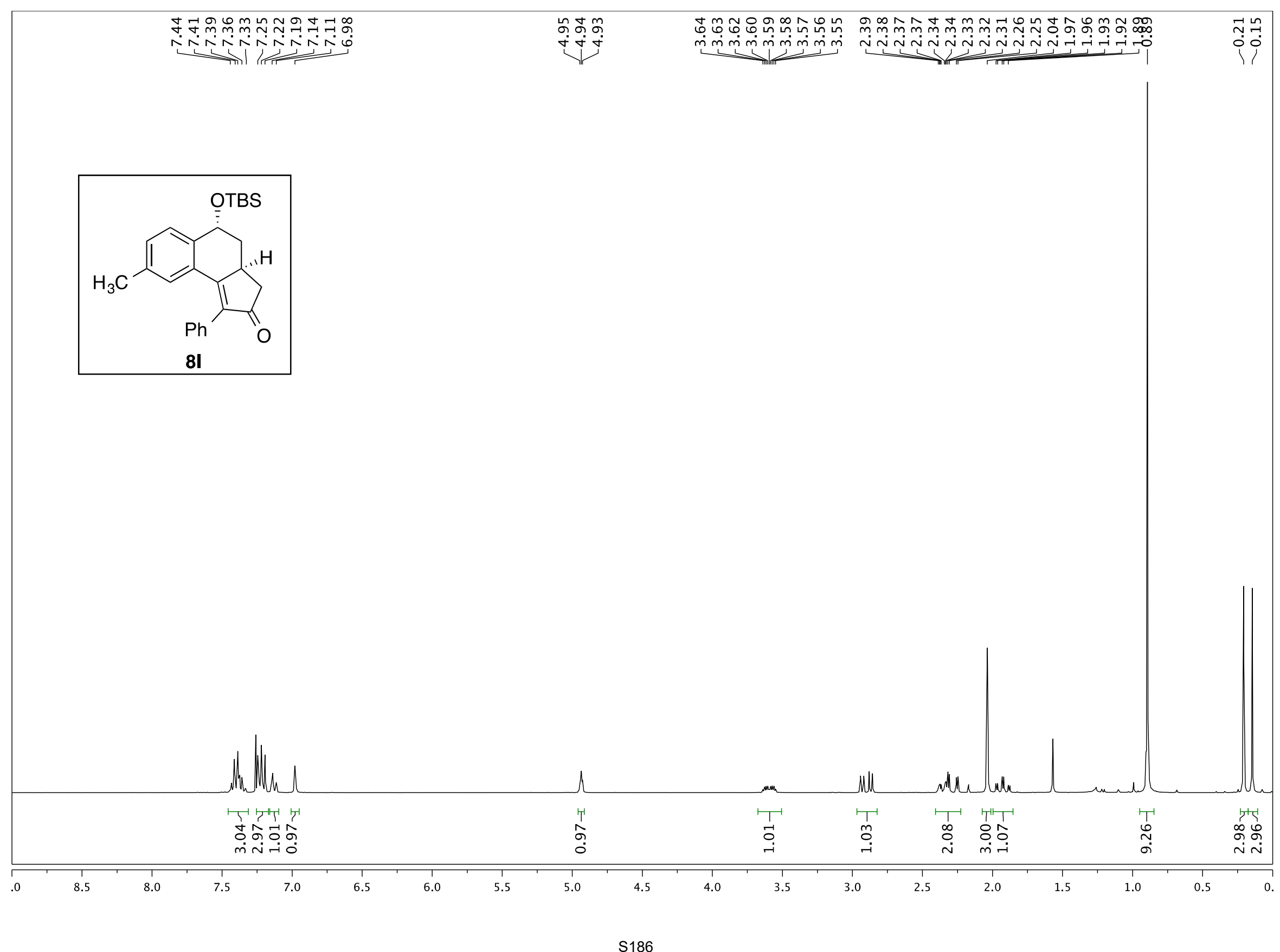




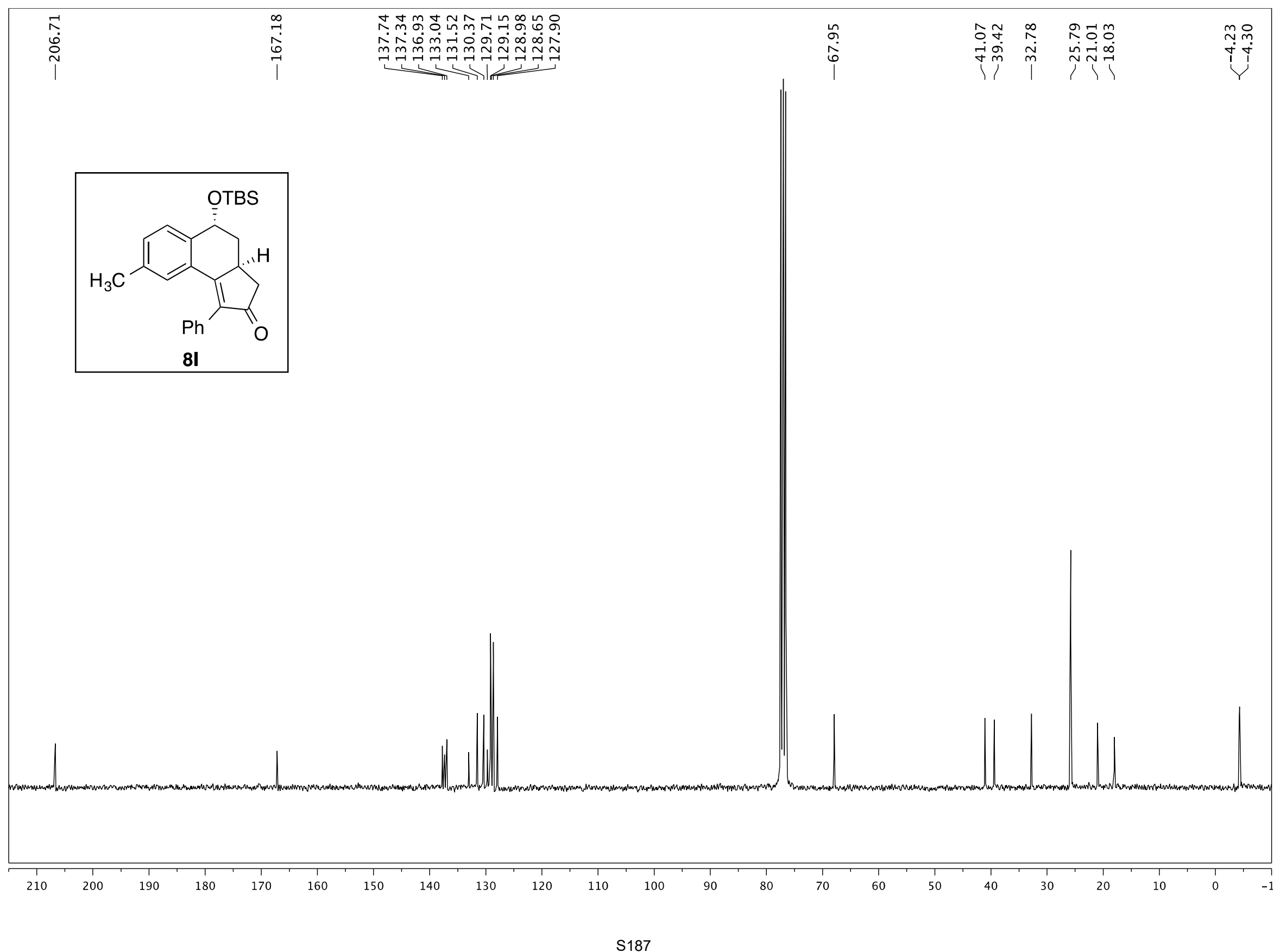




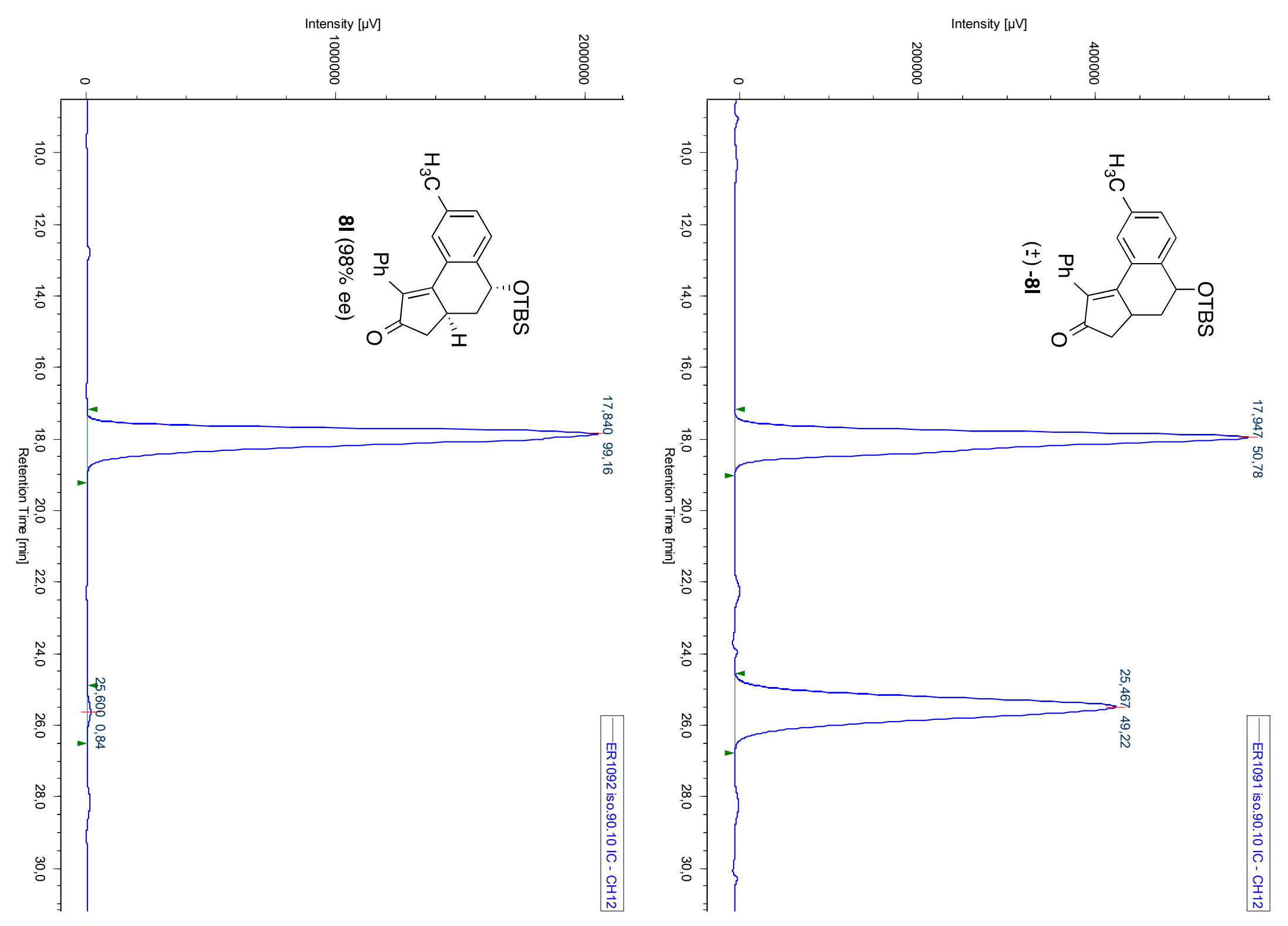




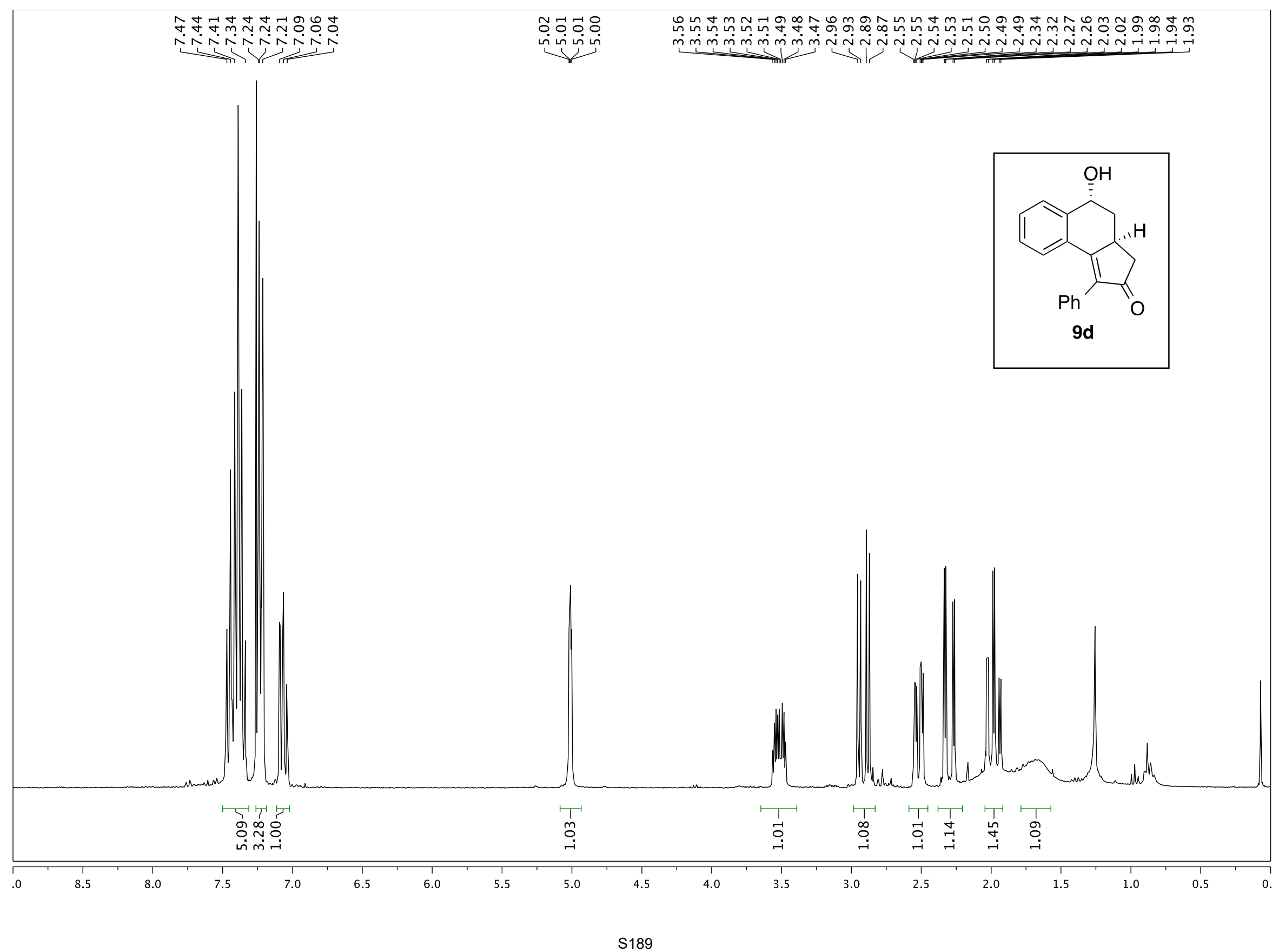




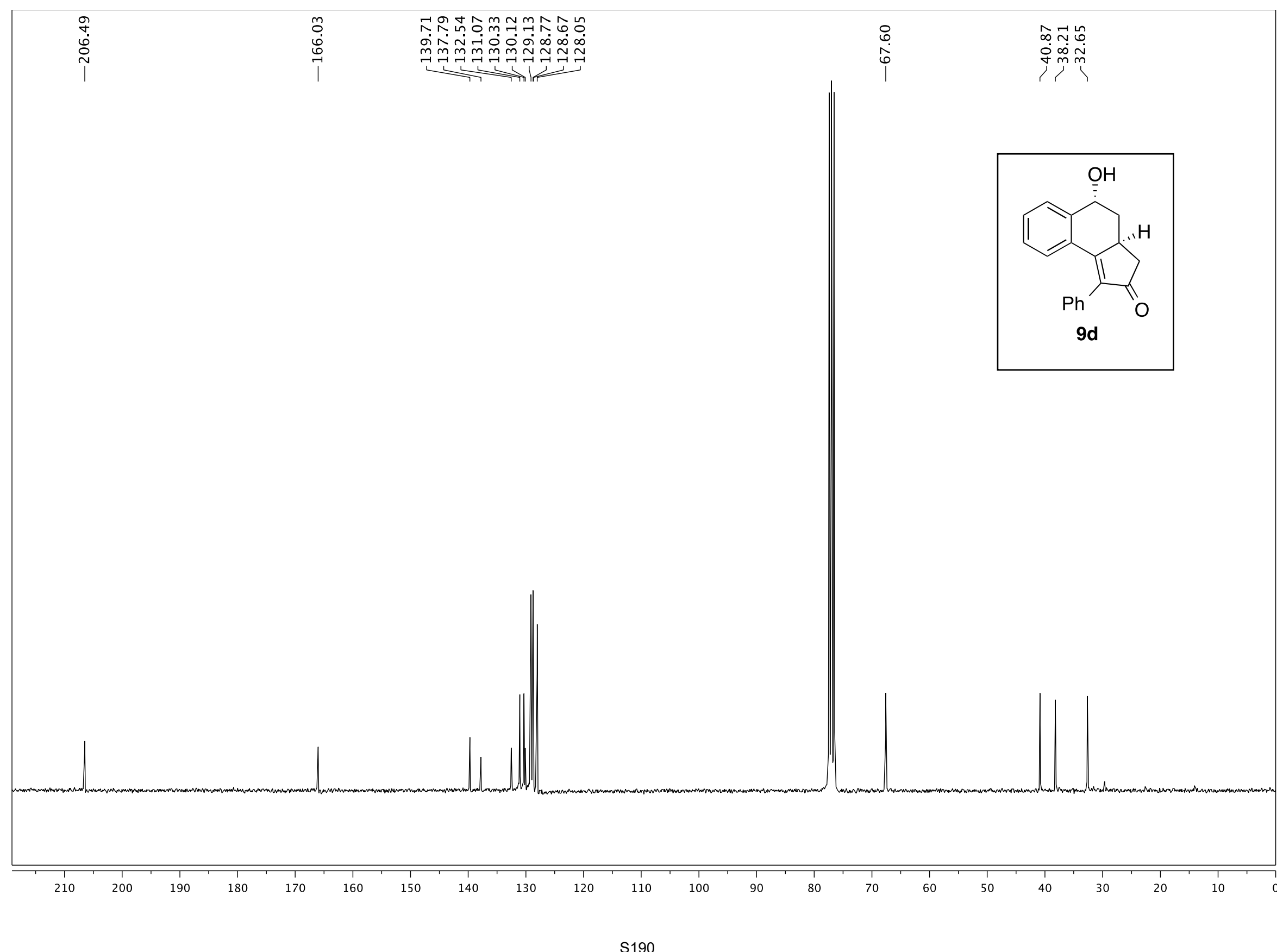




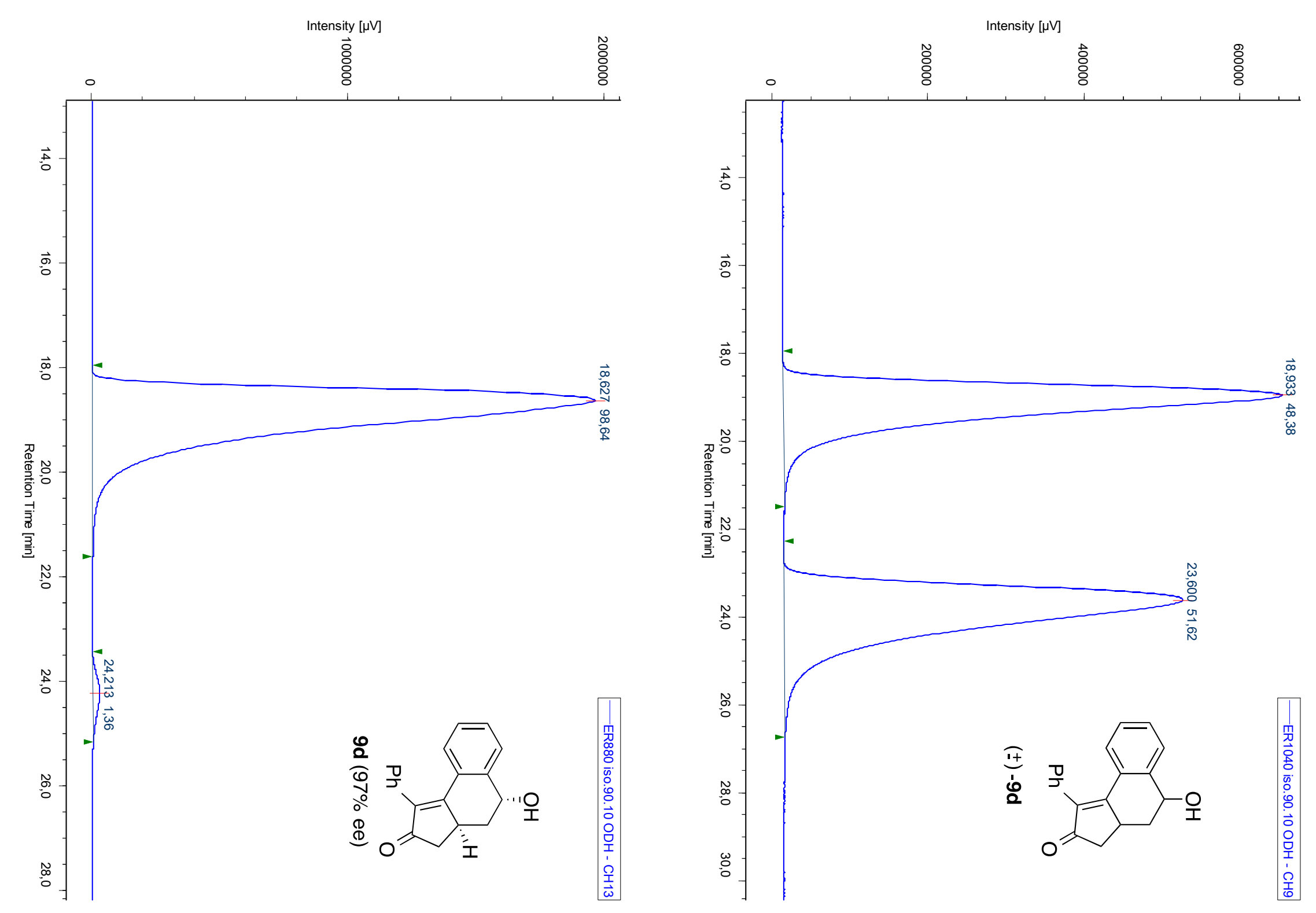




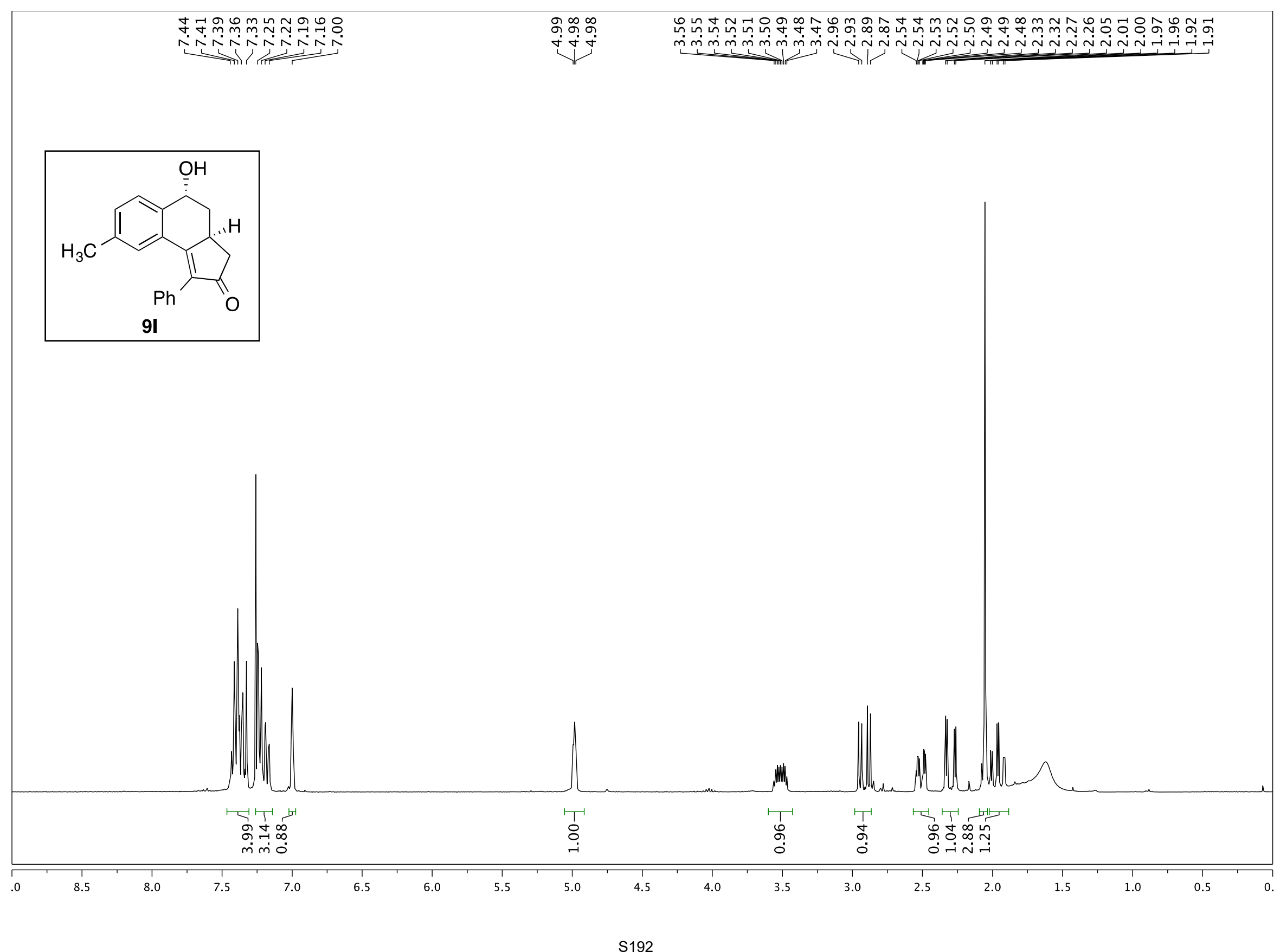




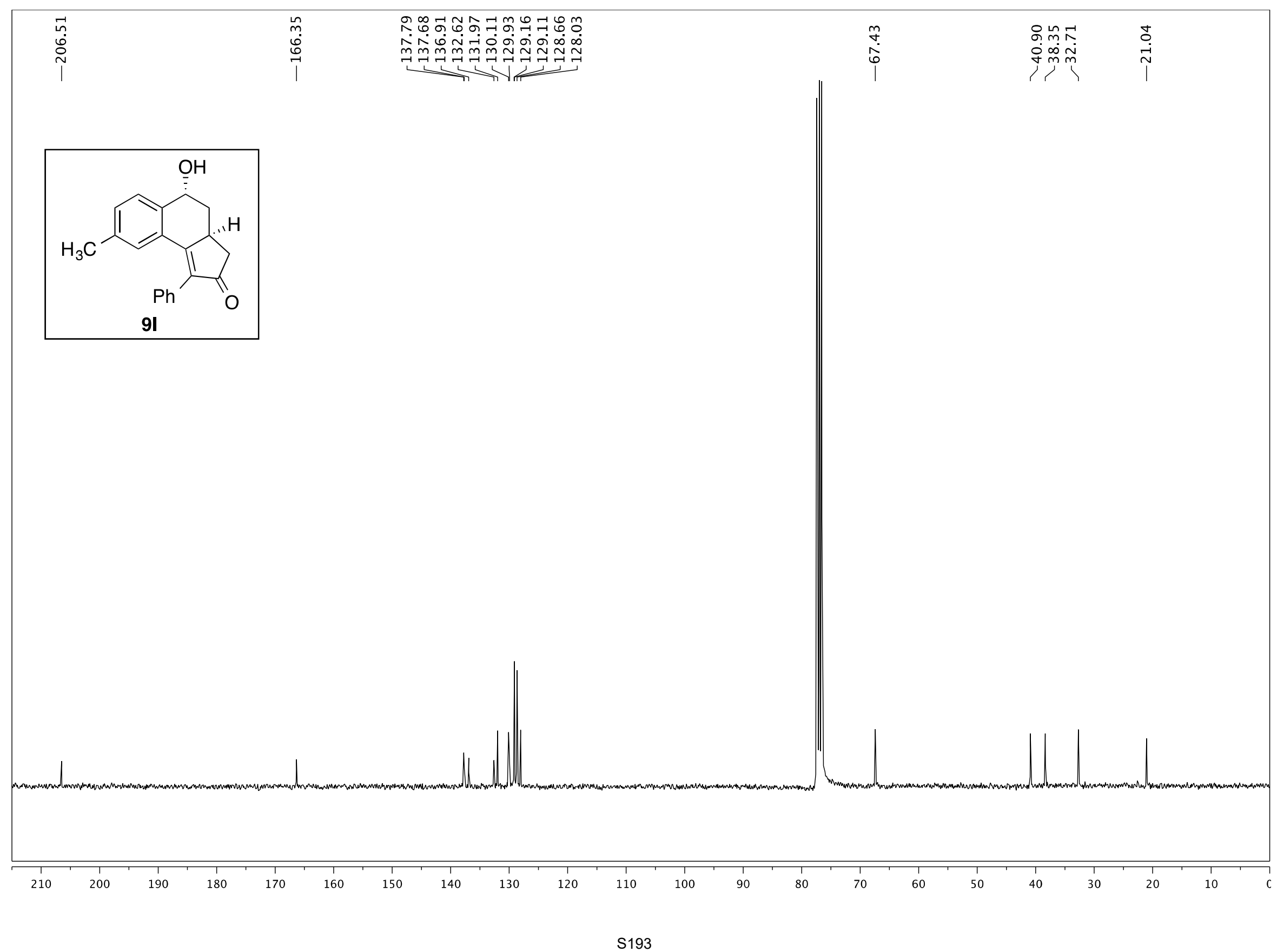




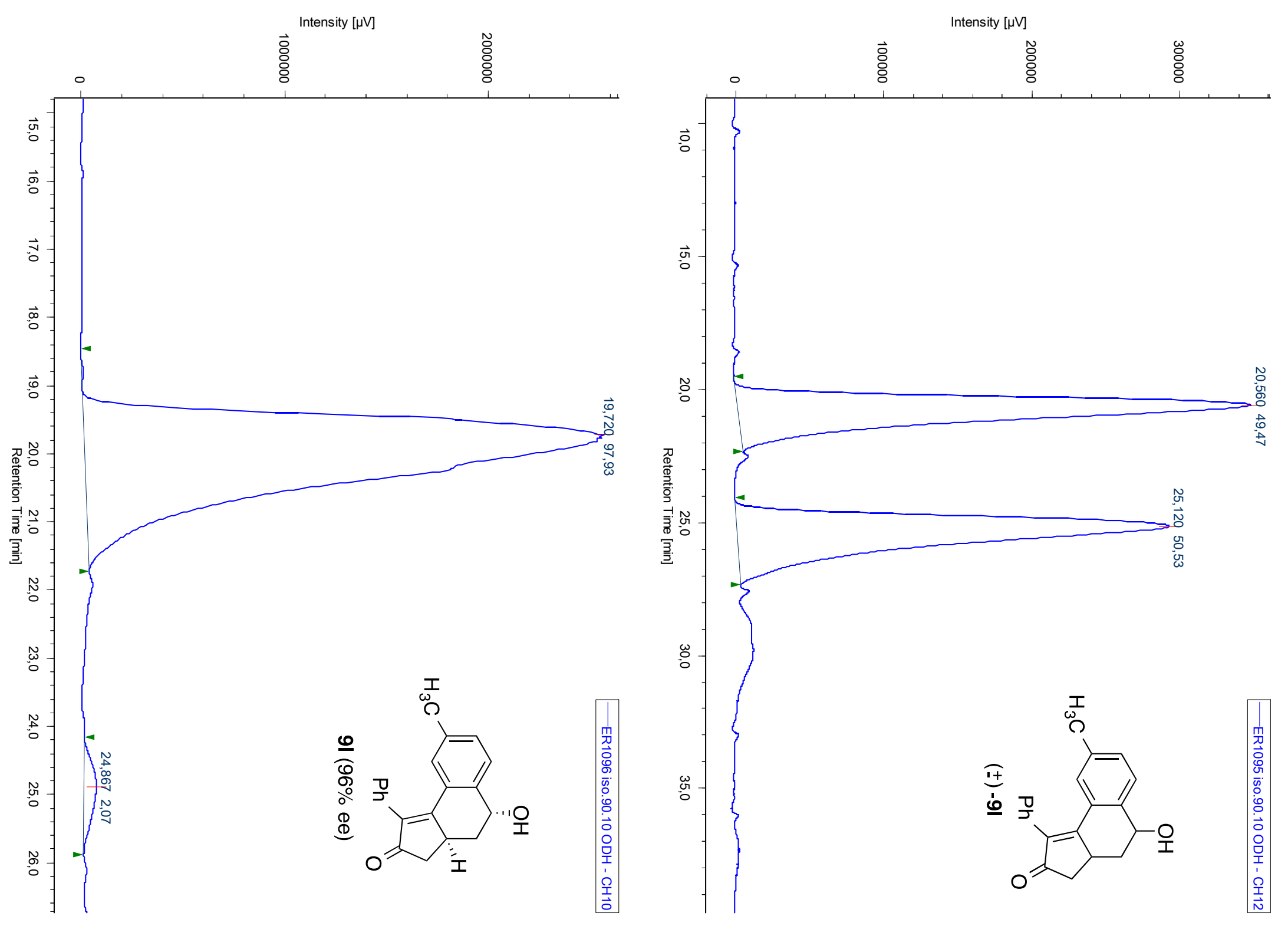




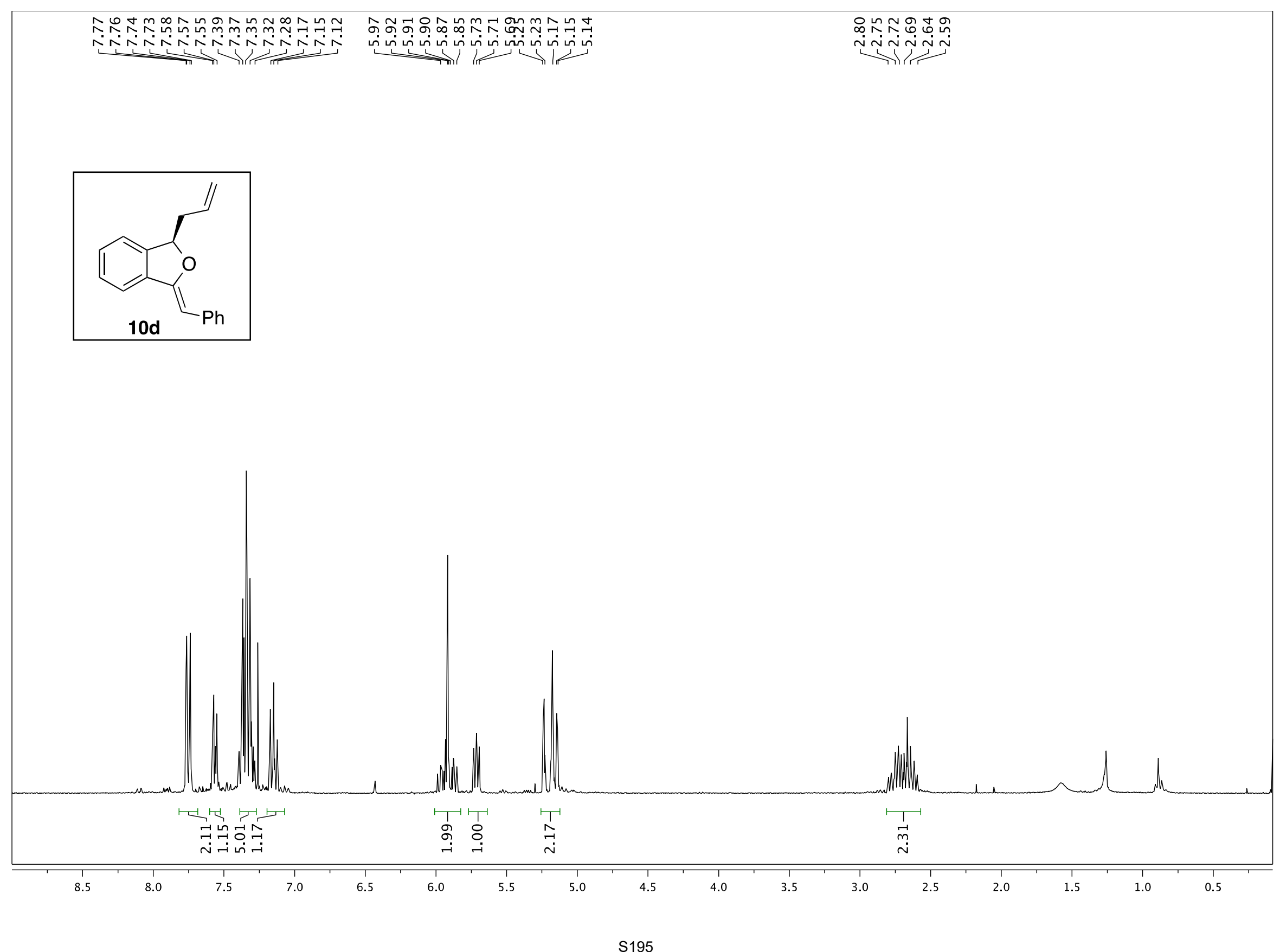




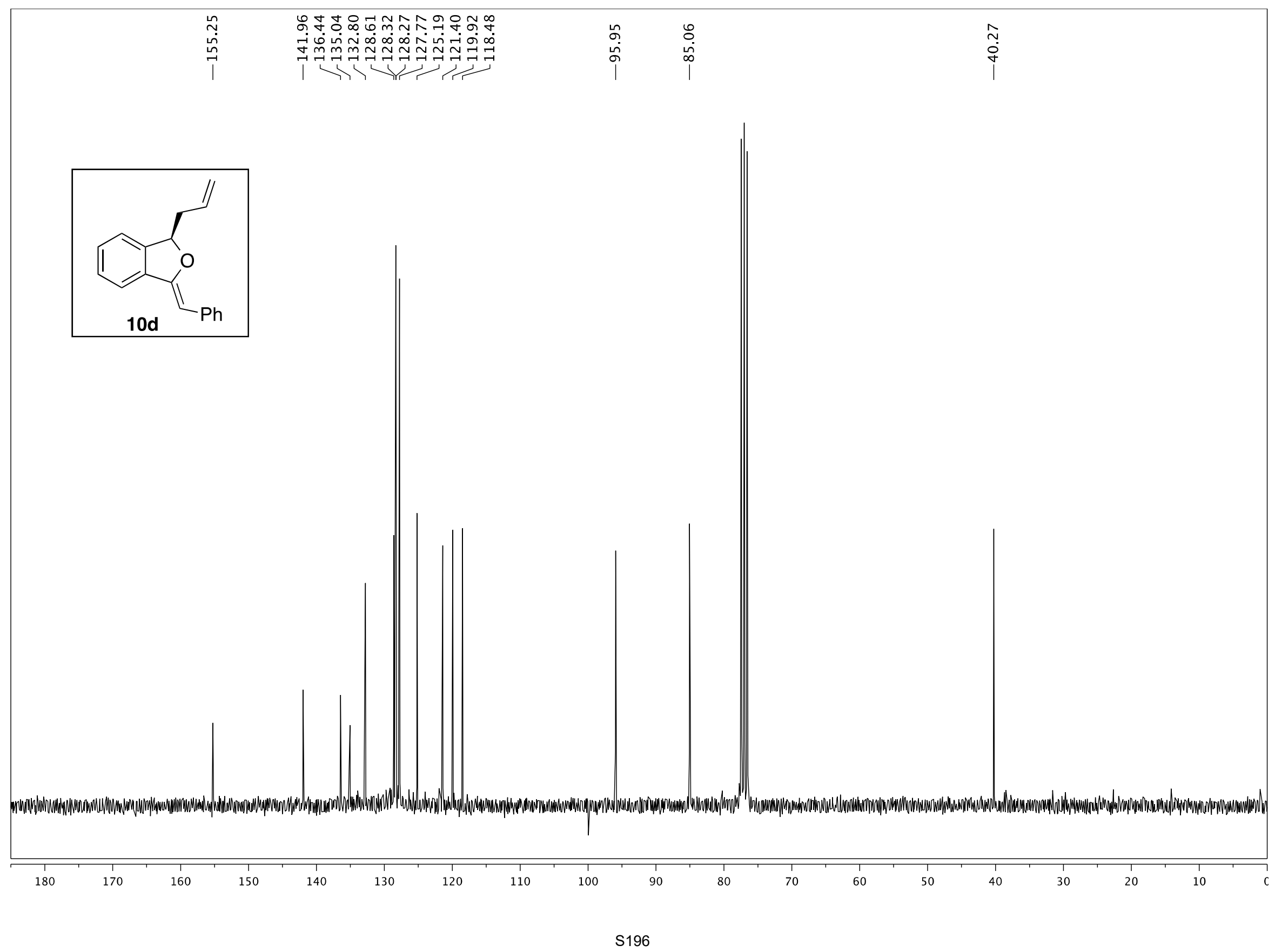




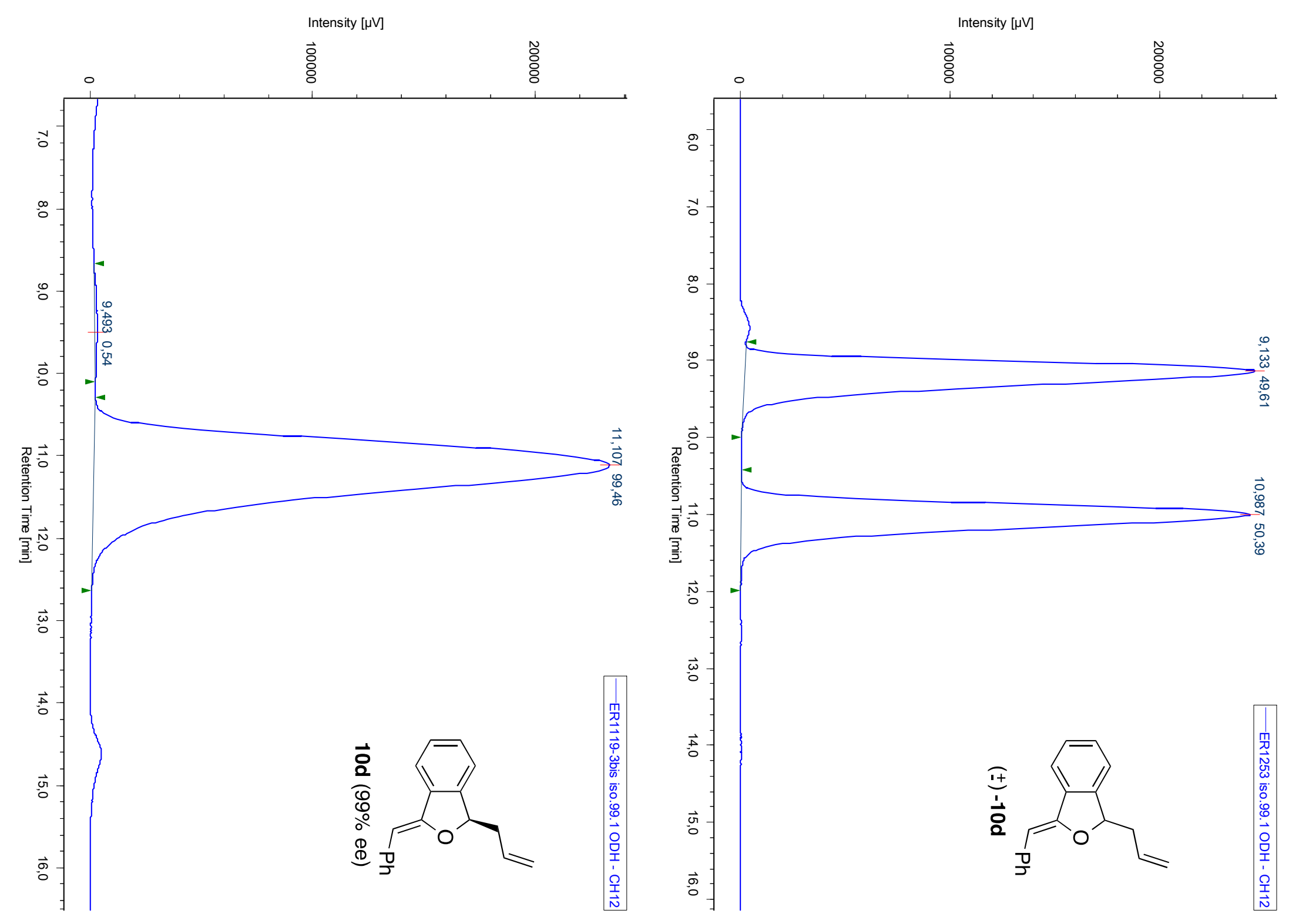




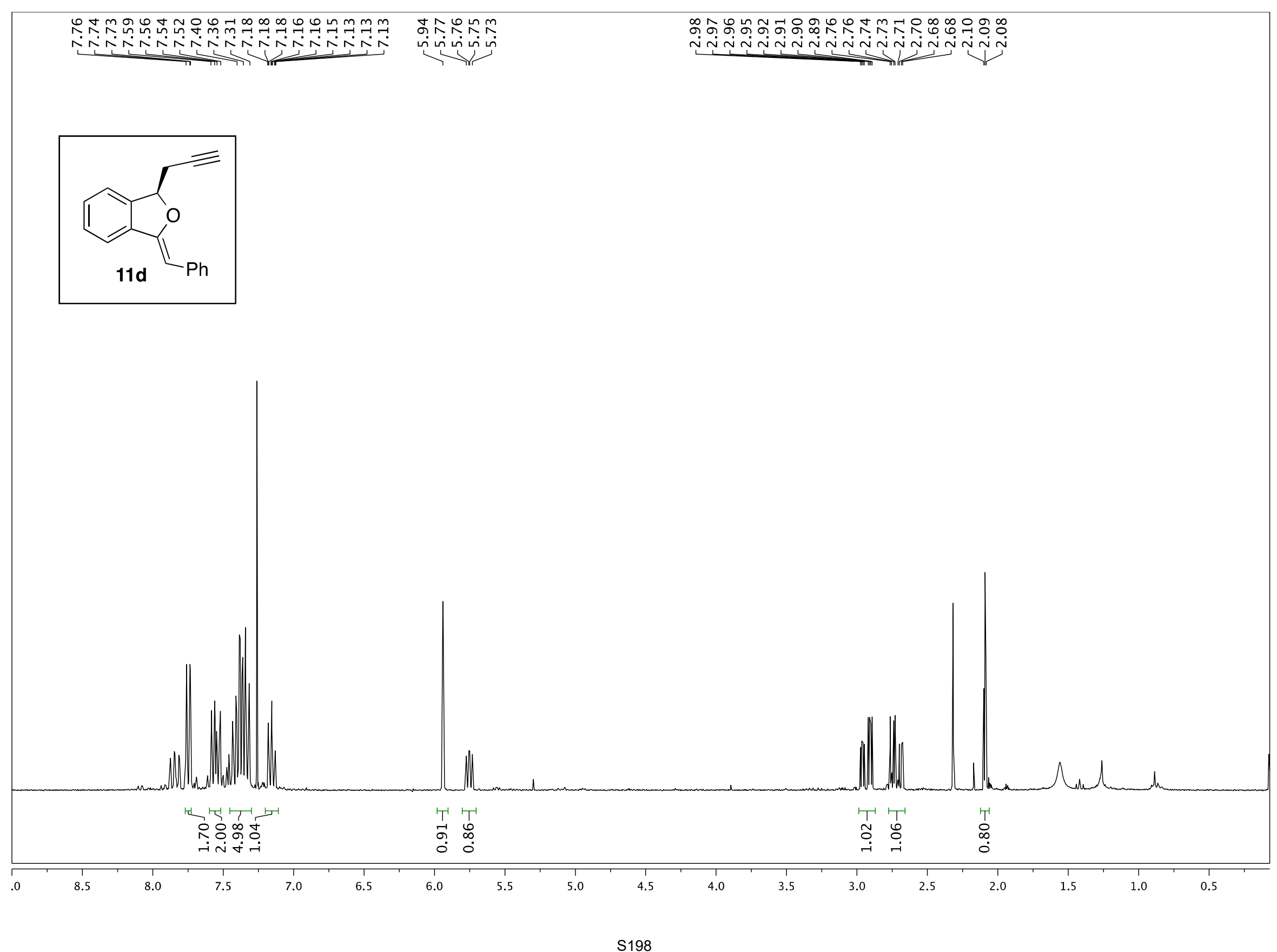




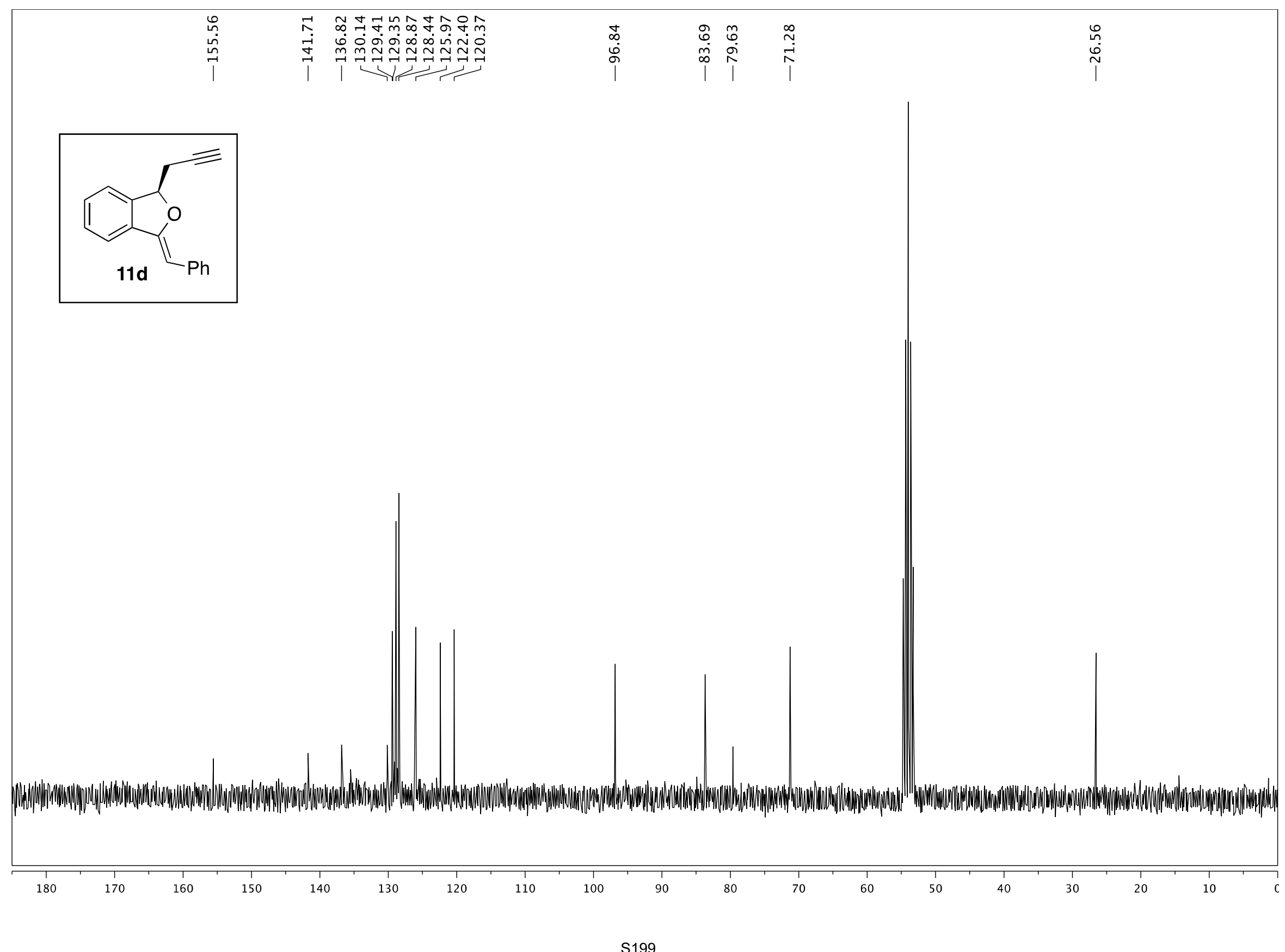




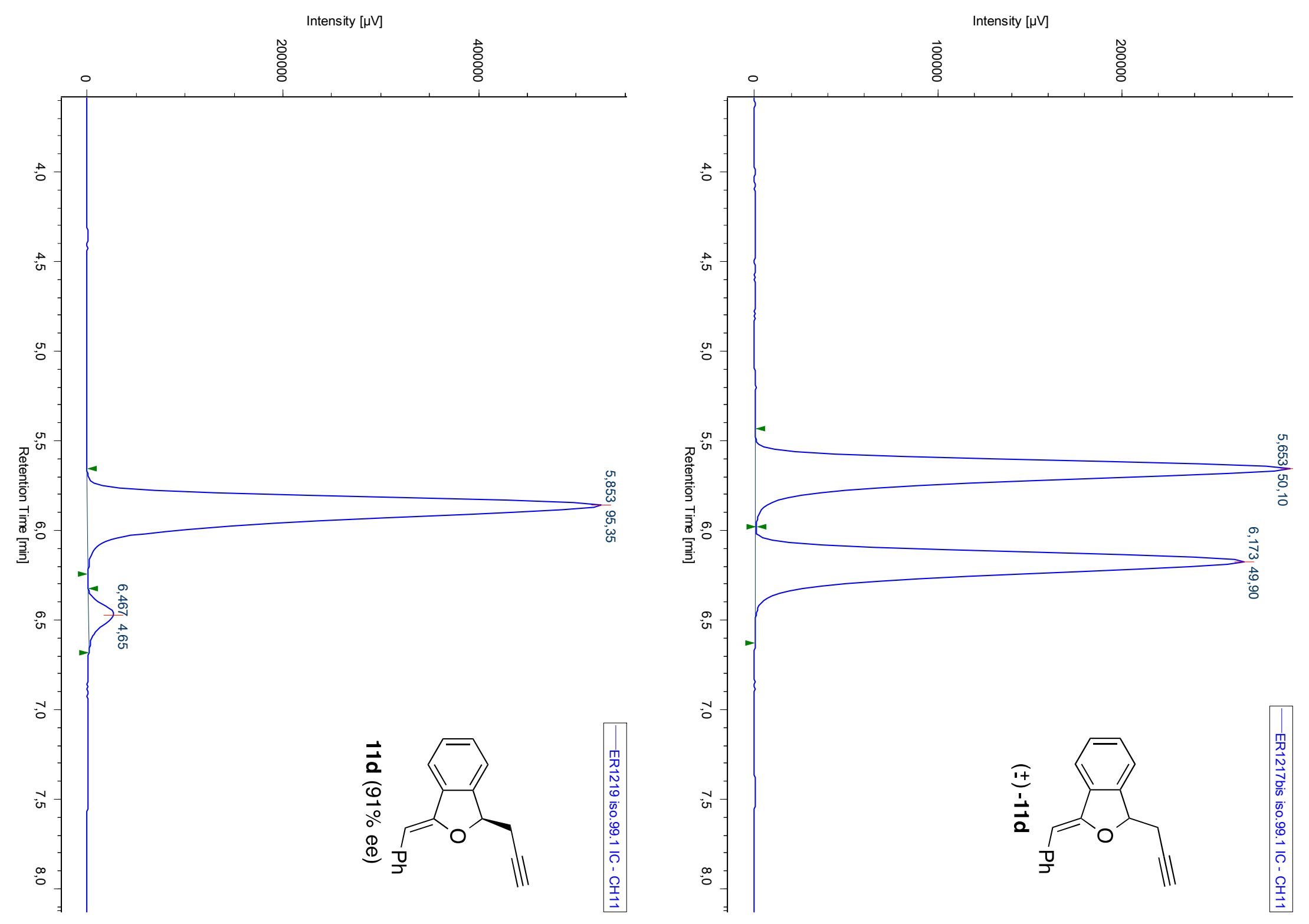

